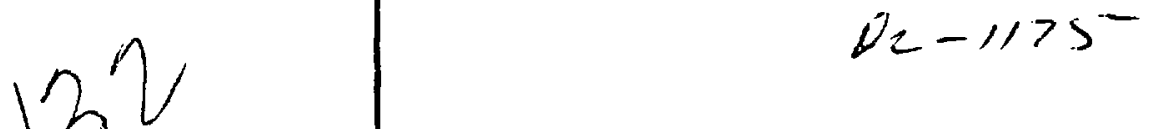

UC. 28

Issued: January 1975

\title{
Quarterly Report on the \\ Medium-Energy Physics Program \\ for the Period Ending October 31, 1974
}

Compiled by

Eleanor Dunn 


\section{An Affirmative Action/Equal Opportunity Employer}

This report presents the status of the LASL Medium-Energy Physics Program. The four most recent reports in this series, unclassified, are:

\section{LA-5518-PR \\ LA-5708-F'R \\ LA-5580-PR \\ LA-5770-.]'R}

In the interest of prompt distribution, this progress report was not edited by the Technical Information staff.

Printed in the Unted Stutes of America Avalcible from Nitinnal Tuchniral Information Service

US Dipartment of Commerce

5285 Port Royal Road

Sprinqteld. VA 22151

Price Prunted Copy $\$ 5.45$ Microliche $\$ 225$

Thu report was prepared os as account of work poserod by the Ualled

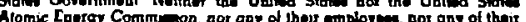

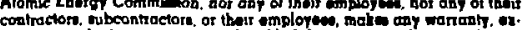

prem of Impliad, of anumen any logal liability or repoascibility for the ox.

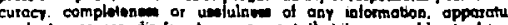

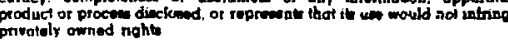

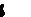


- Notice

This report was prepared as an accolint of work sponsored by the United States Guvernment. Neither the United States nor the United States Energy Research and Development Administration, nor any of their employees, nor any of their contractors, subcontractors, or their employees, makes any warranty, express or implied, or assumes any legal wartanty, express or implied, or assumes any legal liability or responsibility for the accuracy, completeness
or uscfulness of any information, apparatus, product or process disclased or represents that its use would not infringe privately owned rights.

CONTENTS

I. SUMMARY . . . . . . . . . . . . . . . . . . . . 1

II. ENGINEERING SUPPORT . . . . . . . . . . . . . . . . 8

III. ACCELERATOR SUPPORT . . . . . . . . . . . . . . . . 11

IV. ACCELERATOR SYSTEMS DEVELOPMENT . . . . . . . . . . . . . 14

V. ELECTRONIC INSTRUMENTATION AND COMPUTER SYSTEMS . . . . . . . . 22

VI. ACCELERATOR OPERATIONS . . . . . . . . . . . . . . . 29

VII. EXPERIMENTAL AREAS . . . . . . . . . . . . . . . 31

VIII. LARGE-SPECTROMETER SYSTEMS . . . . . . . . . . . . . . . . 43

IX. RESEARCH ........................ . 48

X. PRACTICAL APPLICATIONS OF LAMPF . . . . . . . . . . . . 71

XI. Management . . . . . . . . . . . . . . . . . . . 78

APPENDIX : A. PREPRINT -- LAMPF PROTON LINAC PERFJRMANCE . . . . . . . . 84

B. BI BLIOGRAPHY OF LAMPF-RELATED PUBLICATIONS . . . . . . . . 92 
QUARTERLY REPORT ON THE MEDIUM-ENERGY PHYSICS PROGRAM

FOR THE PERIOD ENDING OCTOBER 31, 1974

\section{SUMMARY}

Engineering Support

The heads from Tanks 3 and 4 of the $201-\mathrm{MHz}$ linac were removed and the drift tubes were checked for optical and magnetic alignment. The drift-tube sten bellows were found to have been subjected to serious overheating. A new design was made and work is under way to replace these bellows during the January shutdown.

Work on the Weapons Neutron Research Facility (WNR) line is progressing satisfactorily. Magnets are being received, tested, and fitted with alignment fixtures; stands and other devices are being fabricated. The floor plates are being installed, and the vacuum envelope for this line is being fabricatel. The slow kicker has been received and it has been tested up to $200 \mathrm{~A}$. The assembly of the fast-kicker power supply has begun.

The ion-pump rebuild facility is now operational. Nine of the large $2400-l / s$ ion pumps were rebuilt and eight of these are now in operation on the accelerator. Although the facility is still being improved upon, pumps are being rebuilt on a regular basis.

The klystron-rebuild facility is being improved by new procedures, additional jigs and $f i x-$ tures, a new vacuum system, and additional personnel.

Some 20 experiments are currently receiving support from the design, fabrication, and alignment sections.

Considerable effort was spent to ready the biomedical facility for the first human biology experiments.

\section{Accelerator Support}

This is the first seport for the accelerator support group which was formed July 1, 1974. Considerable time and effort has gone into organization and staffing of this group. At this time, these goal, are nearing completion with organization established and staffing within about $10 \%$ of the original plan.
The accelerator was operated for over $2100 \mathrm{~h}$ this quarter with about $448 \mathrm{~h}$ of downtime. About two-thirds of this downtime occurred in $201-\mathrm{mbiz}$ systems. The major failures that occurred were a 7835, two 4616s, and three tank windows. The tank problems contributed most of the downtime because of problems with marginal vacuum pumps and tank vacuum leaks during pumpdown after the windows were replaced. The vacuum pumps are being replaced with rebuilt units and this problem should be eliminated in the near future. The tank leaks from the soft-to-hard vacuun will be repaired after the start of the shutdown in Late December.

The 7835 had over $11000 \mathrm{~h}$ on it at the time of failure, which is more than the expected life. Extra downtime was accumulated here and also for the 4616 failure because of required conditioning of the new units. This source of downtime should also be eliminated when the $201-\mathrm{MHz}$ test stand is completed this winter. This test stand will allow preconditioning of all 201- $\mathrm{MHz}$ spare tubes prior to installation.

Accelerator Systems Development

A series of high-intensity runs at $100 \mu \mathrm{A}$ and $200 \mu A$ were conducted. An acceptable bean-spil: pattern in the machine was obtained with the $100-\mu \mathrm{A}$ beam. Preliminary alignment checks were made on the quadrupole lenses in Tanks 3 and 4 and acceptance calculations using the observed misalignment errors have been carried out. Analysis and simulations have been done to try to resolve the problem of length errors in the 805-MHz accelerator structures. Several new diagnostic and tuıing programs have been written and put into service.

A continuing effort has be. $n$ carried out in obtaining long-term reliability and stability in the operation of the injector systems. Several significant faults in the $\mathrm{H}^{+} \mathrm{C}-W$ generator were found and corrected.

The $\mathrm{H}^{-}$beam chopper was put into service this quarter and is being used for both physics research and accelerator diagnostic studies. Single-micropulse operation has been demonstrated. 
Work is continuing on providing beam diagnostics for a high-current $\mathrm{H}^{-}$ion source test stand being built by $\mathrm{p}-11$.

The polarized ion injector program continued on a low-priority level. Electronic Instrumentation and Computer Systems

Major effort this quarter has continued in support of the accelerator control system, continued development of experimental beam-line controls, and in support of experimental programs through the LANPF electronic equipment pool and experimental data-acquisition activities.

The upgrading of the existing injector systems is resulting in better signals for beam analysis and more reliable operation of the hardware, particularly the C-W high-voltage power supply. New software was developed for monitoring the flow of programs through the control computer as part of a continuing effort to improve the operating system. The hardware and software associated with the accelerator operator's consoles and with providing operating information to experimenters continue to be upgraded to provide easier and more reliable operation of the tacility.

Work was initiated t' upgrade the Line A beamdiagnostic instrumentation and the handling of data for presentation to the operatur. More requirements for Line A-l target cell instrumentation have been received and integrated into the sreat Shutdown planning. The controls for the Area A test channel off target A-1 are being implemented. The instrumentation and controls for the Energetic Pion Channel and Spectrometer Systems (EPICS) and the HighResolution Proton Spectrometer (HRS) lines are in the final design for present criteria. Software for control of the secondary beam 1 ines continues to be developed. Applications programs for the biomedical control systems were exercised during the patient treatment this quarter. These programs have required a substantial development in the computer operating system software which has taken place over the past two years.

The experimental data-acquisition lardware and nuclear instrumentation modules have received substantially more support this quarter, to the benefit of the experimental activities. An integrated effort to support existing data-acquisition software and to design new programs was started.
Accelerator Operations

The accelerator was in operation for the entire quarter. There was a total of 276 shifts, for an average of 21 shifts per week.

As in the previous quarter, the machine was operated in 14-day cycles, each consising of 12 shifts for accelerator development, 6 for tuning the beam preparatory to research use, and 24 for research. of the total number of operating shifts, $56 \%$, or 156, were scheduled for research. Machine availability during shifts scheduled for research averaged $70 \%$.

All existing secondary beam lines, the External Proton Beam (EPB) line, the Line-B Nuclear Chemistry Facility, and the Radioisotope Production Facility (RADIP) were in use during the quarter. The RADIP rabbit-cube system was employed for transferring active target foils to hot-cell facilfties for the first time. The most publicized of the research activities during the quarter were the pion frradiations of tumors on human subjects.

Average proton beam currents of $12.5 \mu \mathrm{A}$ at 800 $\mathrm{MeV}$ were attained during research runs in the latter part of the period. Proton beams to Area A for experimental use totalled $6800 \mu \mathrm{A}-\mathrm{h}$, as measured at the A-1 target station, up $177 \%$ from the previous reporting period. An additional $470 \mu \mathrm{A}-\mathrm{h}$ went to Area B. Sixty-six target folls were irjeadiated for nuclear chemistry experiments.

Machine time allocated to accelerator development was used for continued studies of problems of h1gh beam-current operation. Tanks 3 and 4 were opened and alignment of the drift tubes was checked. In the process, a serious corrosion problem on the drift-tube vacuum-seal bellows was discovered.

During a 30-s, high beam-current test run to the switchyard beam stop, the average beam current was increased to $100 \mu \mathrm{A}$ without tripping loss monitors. The cest was terminated after $30 \mathrm{~s}$ because of 11mited heat-dissipation capability of the target. The beam current reached $200 \mu \mathrm{A}$ on a subsequent test, but was terminated after a few seconds by spill-monitor trips.

\section{Experimentai Areas}

The emphasis in the experimental areas for the past few months has been to support a multiple-use research program and, at the same t:ime, to develop the plans and the staff required to achieve the 
objectives of the Great Shutdown. The typical number of simultaneous users of the various beams is six, with occasional simultaneous usage for nine or ten experiments. About 40 experiments have received beam through October 31, 1974, not counting the biomedical program or parasite physics experiments.

Preparations for the prolonged shutdown are under way. The staff ot the Experimental Areas Group (MP-7) has been increased, and a large team of engineers and designers is busy on plans for the targeting, remote handling, and shielding needed for high-intensity operation.

The process water systems sre now operating smoothly, after difficulties with pump maintenance and water quality. Three more water systems should be in operation by early FY 1976.

Tuning procedures for the main beam line have been improved and systematized. The Accelerator operations Group $(M \mathrm{P}-2)$ is assuming an increasing fraction of this work.

A thorough study of the switchyard optics, diagnostic apparatus, and beam properties is under way. New instrumentation (integrated toroids, fast wire scanners, and improved profile monitors] is being developed; some changes have been adopted in the beam optics which have led to increased beam transmission in the switchyard.

In anticipation of higher jeam currents, more consideration has been given to the problem of heating of beam-line components caused by scattered particles. A computer program for calculating expected heat loads is in routine use for design studies.

A coordinated program of beam tuning and optics calculations is in progress for the Stopped Muon Channel (SMC). This work is continuing, with some improvement in channel pejformar.e al ready obtained. Bids have been accepted for the vacuum system for this channe1.

Substantial progress in installation of the Test Channel has been made, and preparations for tuning the beam are under way.

Some progress has been made on the Radiation Effects and Isotope Production Facility (RADIP). Several irradiations were made for neutron dosimetry and checks of the beam position. The neutron dosimetry studies gave rise to a predicted neutron flux of $2 \times 10^{13} \mathrm{n} / \mathrm{cm}^{2}-\mathrm{s}$ for $1 \mathrm{~mA}$ of $800-\mathrm{MeV}$ protons incident on the beam stop. A demonstration of the RADIP Facility is planned before the December shut down. Improvements in the stringer design are needed; eventually, it will be necessary to rework the stringers or to replace them.

Conceptual designs of the target boxes and collimators for target cells $A-1$ and $A-2$ have been completed. The fabrication of the A-l target mechanism is nearly complete, and the A-2 target system is nearly ready for bid. Design studies for a new version of the main beam stop are continuing. Design of tooling and lifting fixtures for remote handling has started.

The nisk that is necessary to make Merrimac operational is proceeding. The hot cells are being outfitted with manipulators and window assemblies. The temporary shielding in Area $A$ has been improved to allow routine operation at $10-\mu A$ average beam current. Design of Merrimac doors, target cell shielding, and secoldary beam shielding is in progress. A roof was added to the experimental cave of the External Proton Beam (EPB), and a Faraday cup was installed in the EPB beam stop. Two major contracts for shielding material (one for concrete and one for steel) have been negotiated; delivery is under way.

An extensive PERT network has been developed to aid in planning for the shutdown; PERT, which is not yet complete, contains 800 separate activities as signed to 47 responsible individuals. About 98000 man-hours of effort will be required to carry out the work packages already identified. The goals are to: stop accelerator operations on necember 24, 1974 ; นurn back on for accelerator development on May 1, 1975; pass beam through Area $A$ on June 1, 1975; and have the capability of supporting a high-intensity research program by July 1, 1975 . Large-Spectrometer Systems Eneryetic Pion Channel and Spectrometers (EPICS)

Channel magnet IP-BM-02 was reassembled during this quarter. In order to complete the vacuum system for this magnet, it was necessary to develop a new seal between the pole tip and the vacuum chamber.

Substantial progress has been made in preparing for the major effort in field mapping and measuring which will be necessary during the next quarter. 
The rapid mapper has been reworked and c.n now be run for an extended period. A change in the electronics allows us to make a complete map in two hours. The alignment procedure has been modified to include enough redundant information so that the modest number of errors which inevitably occur can be found and corrected. The field analysis and shimming codes have been modified to include all field-map information. A shimming procedure has been devised which allows reasonable (two-day) turnaround so that several ite ations are possible. Finally, we have impr'ved the stability of the power supplies and have determined a field-setting procedure which is rapid and gives adequately reproducible results.

The plans for installation of the channel in the shielding have progressed rapidly. Work is proceeding on a design for canyon fill. Final design of all magnet supports has been completed, and the separator support and removal system is being designed.

Substantial results have also been achieved on our lower priority projects. The spectrometer modifications required for in-place assembly and for utilizing the new vacuum chamber seal are under way in the shops. A method of shimming the spectrometer quadrupoles to eliminate the ubserved octupole component has been developed. The assembly stands for the spectrometer have been completed. A $12.7 \mathrm{mcm}$ gid: mount has been obtained to allow $\mathrm{H}_{t}$-winding installation without tying up the crane duriag the Great Shutdown.

High-Resolution Proton Spectrometer (HRS)

Beam Line $C$ is essentially complete and could be used to deliver full beam to the dump except for lack of acceptable shiclding. Magnet LC-BM-04, the fir: : big analyzing magnet in the line, has been shimmed to give an effective field distribution corsistent with design requirements and the remaining beam-running cycles before the accelerator shutdown will be used to determine the resolution of the line and test optimization procedures. The second speitrometer dipole, LC-BM-D2, has been cleaned-up and reworked and is now in the final stages of reassembly. Several moditications and inprovements to this system have been made. Among other things, a gap-height uniformity consistent with the design resolution of the spectrometer has been achieved for the unassemled dipole. Electrical, plumbing, and air-conditioning services for the counting house were completed and the PDP-i1/45 dataacquisition and control computer was received and installed. In addition, other items which received attention during this period include the scattering chamber and sliding-seal curtain-retractor mechanism, the NMR drive and control system, tests with strippers and scanners, tests of power supply stability, and a number of different magnetic field measuremerts and calihrations.

Research

A total of 40 experiments had data-taking runs during the quarter. Among them may be mentioned Exp. 96 (ip elastic scattering at low energy) (LASL/ Arizona State Univ.) which accumblated over 10000 events of intercst. Data were taken for $\pi^{+}$and $\pi^{-}$ at $30,40,50$, and $70 \mathrm{MeV}$. The results from a preliminary analysis lovik promising. Experiment 131 $\left(\pi^{+} d \rightarrow p p\right)$ had a sircessful run (Univ. South Carolina/ ORNL/Virginia Polytachnic Institute/LASL), and complete angular distritutions at 40,50 , and $60 \mathrm{MeV}$ will ts fort'scoming. The Exp. 34 (Univ. Virginia/ LASL) collaboration male measurements of elastic scattering from deuterium for pion momenta of 350 , 450, 540, and $630 \mathrm{MeV} / \mathrm{c}$.

Analysis continued on the $\pi-n u c l e u s$ total crosssection data (Exp. 2) taken last spring. Results of preliminary anaiysis indicato that there are interesting isotopic effects in the cross sections.

Experiment 80 determines the real and imaginary parts of the elastic scat from nuclei. At small angles $\left(<10^{\circ}\right)$, the riclear and coulomb terms of the elastic scattering amplitude interfere. The magnitude of the interference is proportional to the ratio of the real ( $\operatorname{Ref}_{s}$ ) to imaginary ( $\mathrm{Imf}_{s}$ ) parts of the $\pi^{-}$-nucleus scattering amplitude. The Imf $f_{s}$ can be obtained from the total cross section through the optical theorem which allows the real part of the amplitude to be inferred. Phase one of Exp. 80 has been completed. Data on $\pi^{+}$ scattering from ${ }^{40} \mathrm{Ca},{ }^{208} \mathrm{~Pb}$, and ${ }^{12} \mathrm{C}$ at energies from 100-220 MeV on the High-Energy Physics $\left(\mathrm{p}^{3}\right)$ beam have been taken and are in final analysi:s. Muonium measurements (Yale Jniv./LASi/Univ. Heidelberg) have been continued, resulting in improved precision for the hyperfine structure interval 
in the muonium atom $\left(\mu^{+} \mathrm{e}^{-}\right)$ground state. The latest runs, utilizing the separated oscillating fields nethod with low-pressure krypton, give results accurate to $0.5 \mathrm{ppm}$.

Calculations on the E2 nuclear resonance effect in pionic atoms have been performed and several proinising cases have been found. An experiment to detect the effect was run successfully on the biomedical pion channel. This constitutes the first observation of a nuclear resonance effect for hadronic atoms.

The neutron spectra and neutron scactering group (Texas A\&M Univ./Univ. New Mexico/P-Div./Univ. Texas) carried out a number of runs at 647 - and 800 $\mathrm{MeV}$ proton energies. Energy spectra for forward neutrons were ottained for protons bombarding targets of deuterium, beryllium, carbon, and aluminum. Preliminary spectra are shown in the body of this report. In all cases, strong peaks are found corresponding to the quasi-elastic np charge-exchange process. These peaks, interesting in their own right, make possible monergic, low-emittance neutron beams at $8 \mathrm{G0} \mathrm{MeV}$ and lower energies -- an important research facility at LAMPF. In aluminum, a shoulder appearing below the main peak suggests a possible interpretation of an excitation of the giant quadrupole resonance in the residual nucleus. Preliminary discussions of this work were given at several scientific meetings last summer.

Neutron spectra were obtained from the reaction $p+p \rightarrow n+p+\pi^{+}$in the forward neutron direction at $766 \mathrm{MeV}$ incident energy. The neutron spectra, shown in the body of this report, show a peaking of the $\left(\pi^{+}\right)$invariant mass near $1210 \mathrm{MeV}$, rather close to the $\Delta(1236)$, and the shape agrees well with calculations by utephenson, Gibbs, and Gibson.

Experiments by several research teams were performed during the quarter, dealing with aspects of the emission of light fragments from nuclei in connection with the absorption of pions and protons. The techniques employed were various. They included observation of the fragments by TOF and energy-loss measurements, observation of $\gamma$-ray lines from excited states of the residual nuclei, radiochemical separations, and magnetic and TOF analysis of backemitted fragments. For interpretation, a variety of mechanisms were invoked, ranging from simple evaporation through internucleon cascade processes all the way to a hoped- Eor scattering for the LeeWick particle "condenson." The enthusiasm among the practitioners seems to $b c$ infecticus.

A paper has been accepted for publication in Phys. Rev. Lett. dealing with Exp. 137 (A), "A Searcl for Parity Conservation in p-p Scattering" (LASL/ Univ. Illinois). The stated result, namely that the PNC fraction is $(1 \pm 4) \times 10^{-7}$, represents an $j m-$ provement of 4 orders of magnitude over all previous scattering experiments. It is consistent with predictions based on modern current-current theories of the weak interactions and pp phase shifts, but inconsistent with the (np, dr) result of Lobashor et al. The work is continuing with the goal of reducing the standard deviation another factor of 5 .

A second experiment (LASL/Univ. Illinois/Univ. Chicago), carried out with $6.0-\mathrm{GeV} / \mathrm{c}$ polarized protons on beryllium, indicates that the PNC effect is of the order of $10^{-5}$ or less. This again is consistent with current weak-interaction ideas, but is the only measurement of the hadronic ncnstrange part of the interaction available at higher energy. Calculations by Brown indicate that the proton energies available at LAivPF are especially interesting for this type of experiment. The practical experience gained in the above experiments is being incorporated into the design of the LAMPF polarized ion source.

Practical Applications of LAMPF

During the last quarter major emphasis was placed on preparing the biomedical facility for human biology experiments using negative pions for radiotherapy. This effort included hardware and software development, beam collimator design and fabrication, treatment room modifications, and dosimetry and biology experiments. Three patients have received fractionated pion radiotherapy thus far.

Other experimental programs completed or in progress include microdosimetry measurements in the pion beam, observations of the mu-mesic $x$-ray spectra from various compounds and tissue, biomedical channel tuning, and the visualization of the stopping pion distributions. Effort continued in the development and field testing of equipment for localized-rf-current tumor therapy and differential breast-temperature recording. 
Management

Starting with this report, there will be a section devoted to management concerns, administrative policies and procedures, and information of primary interest to LAMPF users. This section will specifically address personnel levels, budgets, safety topics, schedules and related matter, e.g., titles of internal reports and of mailings will be listed so that interested users will know about them. This will supplement but not displace the LAMPF users, Newsletter.

\section{Publications}

S. Shlaer, "Án MBD Primer," Los Alamos Scientific Laboratory report LA-5511-MS (August 1974).

J. K. Studebaker, "A Fast Versatile Preamplifier for Use in Particle Physics, "Los Alamos Scientific Laboratory report LA-5\%49-MS (October 1974).

Clark Goodman, "Neutron Entrgy Flux and Dosage in the LAMPF $\pi^{-}$Biomedical Channel," Los Alamos Scientific Laboratory report LA-5746-MS (October 1974).

R. D. Stambaugh, D. E. Casperson, T. W. Crane, V. W. Hughes, H. F. Kaspar, P. Souder, P. A. Tholipson, H. Orth, G. zu Putlitz, and A. B. Denison, "Muonium Formation in Noble Gases and Noble-Gas Mixtures," Phys. Rev. Lett. 33, No, 10, 568 (1974).

C. J. Orth, W. R. Daniels, and B. J. Dropesky, "Identification of $236 \mathrm{Th}, "$ Phys. Rev. C 8, No. 6, 2364 (1973).

H. A. Bethe and M. B. Johnson, "Dense Baryon Matter Calculations with Ralistic Pctentials," Nucl. Phys. A230, 1 (1974).

R. L. Hutson, "In Vivo Tissue Analysis Using MuMesic X Rays," Trans. of Amer. Nucl. Soc. 18, 98 (1974).

J. M. Potter, J. D. Bowman, C. F. Hwang, J. L. McKibben, R. E. Mjschke, D. E. Nagle, P. G. DeBrunner, H. Frauenfelder, and L. B. Sorensen, "A Test of Parity Conservation in p-p Scattering," Phys. Rev. Lett. 33, 1307 (1974).

R. A. Tame'son, R. S. Mills, R. L. Cady, "Performance of Pulsed $805-\mathrm{MHz}, 1.25-\mathrm{MW}$ Klystrons into Mismatched Leads," Los Alamos Scientific Laboratory "eport LA-5649 (September 1974).

R. A. Jameson, R. S. Mills, M. D. Johnston, "Management Information for LAMPF," Los Alamos Scientific Laboratory report LA-5707-MS (August 1974).

Papers Prepared for Publication: Papers Submitted at Conferences

M. A. Paciotti, J. N. Bradbury, J. A. Helland, R. L. Hutson, E. A. Knapp, O. M. Rivera, H. B. Knowles, $G$. Pfeufer, "Tuning of the Biomedical Channel at LAMPF," to be presented at the 1975 Particle Accelerator Corf., Washington, DC, March 12-14, 1975.
Jcseph R. Parker and Donald J. Liska, "Calibration of the Beam-Spill Control System at LAMPF," ibid.

Donald J. Liska, "The Particle Separator at Los Alamos," ibid.

Patrick A. Thompson, "Heating by Secondary Particles at Meson Factories," ibid.

Patrick A. Thompson, W. P. Lysenko, and P. Gram, "LAMPF Muon Channel Operation," ibid.

W. P. Lysenko, P. A. Thompson, H. Vogel, and 3. Ohnuma, "Beam Calculations for LAMPF Muon Channel," itid.

G. R. Swain, "Increasing the Relevance of Data Presented to the Operators in an Accelerator Control System," ibid.

Richard D. Werbeck and Robert J, Maceli, "Performance of the High Energy Pion Beam at LAMPF," ibid.

01 in B. van Dyck, A. Harvey, H. H. Howard, and Dennis Roeder, "Instrumentation Operating Experience on LAMPF Main Beam Lines," ibid.

H. O. Meyer, G. G. Ohlsen, R. A. Hardekopf, R. V. Poors, and J. W. Sunier, "Preliminary Measurement of the Projectile Spin Dependence in the Reantions $\mathrm{d}+{ }^{3} \mathrm{He} \rightarrow \mathrm{p}+\mathrm{t}+\mathrm{p}$ and $\mathrm{d}+{ }^{3} \mathrm{He}+\mathrm{p}+3_{\mathrm{He}}+n, "$ prepared for the intern. Conf. on Few Body Problems in Nuclear and Particle Physics, Laval Univ., Quebec, August 27-31, 1974.

J. M. Potter, J. D. Bowman, C. F. Hwang, J. L. McKibben, R. E. Mischke, D. E. Nagle, P. DeBrunner, H. Frauenfelder, L. B. Sorenson, "A Measurement of Paricy Violation in Proton-Proton Scattering at 15 MeV," ibid.

¿. W. Bjork, P. J. Riley, B. E. Bonner, J. E. Simmons, K. D. Williamson, Jr., D. W. Werren, H. C. Bryant, C. G. Cassapakis, S. Cohen, B. D. Dieterle, C. P. Leavitt, D. M. Wolfe, M. L. Evars, G. Glass, J. C. Hiebert, M. Jain, and L. C. Northcliffe, "Neutron Spectra from Proton Bombardment of Deuterium at 647 and $800 \mathrm{MeV}, "$ ibid.

B. E. Bonner et al, 'Measurement of the $0^{\circ}$ Neutron Spectrum from the Reaction $p+p \rightarrow n+p+\pi$ at $\mathrm{Tp}=764 \mathrm{MeV}, "$ ibid.

L. C. Northcliffe et al., "Precision Measurement of $n-p$ Charge Exrhange Cross Section at $640 \mathrm{MeV}, "$ ibid.

Robert A. Eisenstein, "Exotic Particles as Probes of Nuclear Structure - a Review," invited talk presented at the Div. of Nuclear Physics Mtg., APS, Pittsburgh, November 1, 1974.

R. E. Mischke, "A Test of Parity Conservation in p-p Scattering," ibid.

Louis Rosen, "The Clinton P. Anderson Meson Physics Facility - a New Tool for Basic Research and Practical Applications," presented at the Div. of Plasma Physics Mtg., Albuquerque, M, October 30, 1974. 
M. Leon, J. Bradbury, h. Danie1, J. Reidy, "Observation of the $1: 2$ Nuclear Pesonance Effect in Pionic $11{ }^{2} \mathrm{Cd}$," abstract subnittad for the Ans meeting, Anahein, CA, January 39-February 1, 1975.

Mario E. Schillaci and bennis L. Roeder, "Computer Mocieling of Neutron bose at Various Locations Within the Body During Pion Radiotherapy," Fifth Intern. Conf. on the Use of Computers in Radiation Therapy, Hanover, NH, August 19-23, 1974.

James D. Doss, Phillip Day, Sterling Edwards, Charles W. McCabe, and Cerald K. Heiss, "Localized Current Field Tumor Therapy - Prcliminazy Results with Spontaneous Animal Tumurs," Assoc. for the Adrancement of Medical Instrumentution, Boston, MA, March 16-19, 1975 (to be published in Medical inst rumentation).

P. Souder, D. E, Casperson, T. W. Crane, V. W. thughes, H. F. Kaspar, R. D. Stambaugh, P. A. Thenipson, H. Orth, G. zu Putiitz, A. B. Denisot, and M. Camani, "Depolarization of Negtive Muons in Helium Gas; Search for Muonic lelium Atom," Proc. of the Fourth Intern. Conf. on Atomic Physics, Heide:berg, July 22-26, 1974 .

D. E. Casperson, T. H. Crune, V. î. Hughes, Hi. F. Kaspar, P. Souder, R. D. Stambaugh, P. A. Thompson, H. Orth, G. zu Putlisz, and A. B. Denison, "Initiation of Muonium Magnetic Resonance Studies dt LAMPF," ibid.

E. A. Knapp and J. N. Bradbury, Medical Linac Design Possîbilities," Conf. on Small Acceleraturs, Denton, TX, October 22-24, 1974.

Herbert Daniel, "Calculation of Multiple Scattering of Charged Particles $A l l$ lowing for Energy Loss and a Homogeneous Longitudinal Magnetic Field," submitted for publication in Nuci. Instrum. Hethods.

Kosai Tanabe, "Nuclear Many-Body Effect in Muonic Atom," submitted for publication in Phys. Rev. Lett.

Kosai Tanabe, "Photon Propagation in Nuclear Mat ter," submitted for publication in Phys. Rev.

H. B. Knowles, "An Envelope Equation in the Dispersive Plane of a Bending Magnet," submitted for publication in Particle Accelerators.

J. D. Bowman et al., "A New Limit on Parity Violation in p-p Scattering at $15 \mathrm{MeV}$," submitted for Summer Study on High Energy Physics with Polarized Beams, Argonne National Laboratory, July 22-26, 1974.
R. L. Itutson, "Recent Experiments with Mu-Mesic $X$ Rays," presented at the Rocky Mountain Chapter of the Ainer. Assoc. of Physicists in Medicine, September 27-28, 1974.

J. F. Dicello, T. K. Armstrong, and R. L. Itutson, "Calculations for Dosimetry and Microdosimetry of Negative Fions," to be presented at the Radiological Soc. Mtg., Chicago, December 1-E, 1974.

H. I. Anols, J. F. Nicello, T. F. Lane, G. W. Pfeufer, J. A. helland, and H. B. Knowles, "Microdosimetry of Negative Pions at LAMPF," ibid.

M. L. M. Bonne, J. D. Doss, F. 's. Gerner, E. G. Mayer, R. C. Miller, and W. Conner, "Localized Heating as an Adjunc: to Radiation Therapy," ibid.

M. Leon "i2 Nuclear Resonance Effect in Pionic Aunils," submitted for publication in Phys. Lett.

E. P. Elkins, "Control, Data-Acquisition, and Display Using a Satellite Mini-Computer System," to be presented at Data Communications Interface 74. Dallas. TX. March 25-27, 1974. A1 so vrevared for publication in The Datacomm Planne:-

D. R. Machen, "CAMAC Branch Highway:" and "CAMAC Serial Systen," presented at Stanfcrd Linear Accel. erator Center in conjunction with IEEE SLAC-Sponsored Course on Computer Instrumentation, Ocะober 26, 1974.

B. L. Hartway, "A Tutorial on thman Factors Engineering," presented at the Purdue hiorkshop Industrial Computer Systems, Purdue Univ., October 7, 1974.

J. L. McKibben, Ralph R. Stevens, Jr., Paul Allison, and R. A. Hardekopf, "Polarized $\mathrm{H}^{-}$Source for LAMPF," Symp. on Ion Sources and Formation of Ion Beams, Berkeley, CA, October 22-25, 1974.

Paul W. Allison, Earl A. Meyer, Donald $h$. Mueller and Ralph R. Srevens, $\mathrm{Jr}_{\mathrm{r}}$, "Performance of the LAMPF $\mathrm{H}^{-}$Injector," itid.

R. A. Jameson, "LAMPF Proton Linac Performance," presented at the IV All-Union National Conf. on Particle Accelerators, November 18-20, 1974, USSR Academy of Science, Mcscow, USSR.

C. Hwang et al., "A Measurement of Parity iiolation in Proton-Proton Scattering at $15 \mathrm{MeV}, "$ presented at the Intern. Conf. on Few Body Problems in Nuclear and Particle Physics, laval Univ., Quebec (August 1974). 


\section{ENGINEERING SUPPORT}

LANPF Accelerator

Accelerator Support

The heads on the 201.25-MHz Tanks 3 and 4 were removed and all drift tubes in these tanks were checked for alignment from an opticai-reference line from the 805-MHz linac. An instrumentmonument station was accurately located between these tanks and target stations were set up to establish the reference 1 ine through these tanks.

In addition, the drift-tube quad magnets in Tanks 3 and 4 of the 201-MHz linac were checked. A special Hall-probe fixture was designed and built that would slip into the drift-tube bores. Magnetic integrity of the quads and the relative centerline position of the bore tubes with respect to the quad poles was determined using this fixture. In all cases for Tanks 3 and $4 \mathrm{drjft}$ tubes the magnetic fields and bore-tube concentricities were within design limits.

Work is continuing on spare drift-tube assemblies for the 20:-Miz linac. Workstands for drifttube inspection have also been fabricated.

The $r f$ bellows on the drift-tube stems were found to be partially melted and porous. The rf heating on these bellows was calculated to be excessive for conduction cooling and several alternative designs are being considered to eliminate this heating problem. Several bellows manufacturers have been contacted and the bellows will be replaced during the shutdown which starts in December.

The beam buncher for the $\mathrm{H}^{-}$injector line is neawing completion. It is being readied for the final $\psi$, brazing heat and assembly. The monitor loops, tuning slug, and rf drive loop are on hand. This unit is scheduled for installation during the accelerator shutdown.

\section{$805-\mathrm{MHz}$ RF System}

The VA-862A klystrons have accumulated more than 403000 filament hours while the L-5120s have accumulated 72000 filament hours, One L-5120 failed during this quarter as a result of a puncture in the cathode ceramic occasioned by a modulator tank arc.

The LPT- 14 modulator triodes have accumulated 455000 filament hours. A total of 20 reprocessed $L P T-445$ have been installed in the accelerator and the mean time to failure after reprocessing is $\approx$ $20 \mathrm{co} \mathrm{h}$.

\section{Klystron-Repair Facility}

A major effort to improve the klystron-repair facility was initiated in this quarter. Reciprocal visits were made between the Stanford Linear Accelerator Center and LAMPF klystron groups to compare rebuild procedures and facilities. As a result of these visits and discussions, several new procedures were written and these will be implemented in future rebuilds. An assembly fixture to facilitate the insertion of the cathode subassembly into the $k l y-$ stron is being fabricated. A new pinch-off tool for $1.9-\mathrm{cm}$ tubing is also being fabricated. Bolt-on, pinch-off assemblies for $1.9-\mathrm{cm}$ and $3.8-\mathrm{cm}$ tubulations have been designed and will be used as soon as they are ayailable. These assemblies will eliminate a troublesome silver-soldering operation on subsequent rebuilds. Kovar cups and ceramic rings for the heater insulators have been fabricated. Trial furnace runs were made on the individual components and the assemblies were brazed together.

A substantial quantity of rebuild parts, including heaters, cathode and-mod-anode ceramics, and output windows were ordered. The diffusion-jump system on the bake-out oven has been removed and a turbomolecular pump installed in its place. lnterlock systems for both vacuum systems are also being de. signed. These steps should greatly improve the reliability of the vacuum.

Two klystrons were rebuilt during the quarter, but neither was successful. The first tube, $S / N$ 103, developed a leak at the heater insulator which could not be repaired without disassembly because of the high operating temperature of the insulatox. The second klystron, $S / N 214$, had a partially poisoned cathode after the rebuild. However, it has been operated at the 0.5-MN level for rf parameter checks.

\section{Ion Pump Rebuilding}

Nine of the ter $2400-\ell / 5$ Hughes ion pumps required for the $201-\mathrm{MH} z \mathrm{z}$ linac vacuum system have been rebuilt and eight of these are back in service. The cathodes on seven of these pumps were completely sputtered through, necessitating replacement of all titanium material.

The small vacuum bake-out oven and automatic temperature controller to be used for the $600-\ell / \mathrm{s}$ 
pumps was complezed and checked for proper operation.

\section{Magnet Testing and Metrology Lab}

Al1 of the bending magnets for the Weapons Neutron Research Facility (WNR) have been measured and the tooling holes have been installed and inspected. Alignment mosuments were fixtured to the six bending magnets which will go into the waterfall area.

A $20009-\mathrm{kg}$ ber.ling magnet which is to be used on Exp. 160 was processed through the tooling dock. External alignment maris were placed on the magnet and a locating boss mounted on to the bottom surface.

The Energetic Pion Channel and Spectrometers System (EPICS) bending magnets, BM-02 and -03, were inspected and measured in the tooling dock. Information regarding the location of small mapping holes in the pole pieces with respect to the alignment rionuments mounted or the yokes was obtained.

The EPICS magnetic field-measuring assembly was rechecked and shimmed to proper alignment.

Experimental Lines

Line D--Weapons Neutron Research Facility

The ten tunnel-quadrupole magnets are on hand and seven are ready for installation. Seven of the seventeen quadrupole magnets which are required for the switchyard and waterfall doublets and triplets were received and are being tested. The support structure and alignment devices for these magnets were received and are being assembled. The alignment monuments for the switchyard doublets and triplets and for the tunnel quads are bcing fabricated.

Floor plates for all magnet supports in the waterfall area were installed. The six bending magnets required for this area, along with their mounts, were placed in position and roughly aligned.

A production-type linear actuator has been received, inspected, and found to be acceptable; the vendor has been advised to proceed with fabrication of 15 units. All wire-scanner components to be used with these actuators are on hand. Vacuum boxes for scanners 01 and 02 are on hand and the design of all other scanner boxes is complete.

Considerable effort was expended this report period in the fabrication of vacuum envelopes and related devices utilizing both $\mathrm{H}_{2}$ furnace brazing and inert-gas welding techniques. The beam stop along with its vacuum chamber are complote except for bellows installation. Vacuum beam Iipes for the six waterfall bending magnets were built and installed. Fabrication of beam-line tubulation for the long tunnel run is $90 \%$ complete.

The slow kicker for ine $D$ was received and has been pulsed at low currents (20-A peak) in a closed-loop mode. The initial tests were very successful and a system is now being constructed which will allow pulsing up to $200 \mathrm{~A}$ to test the feasibility of the modulator and control systems at inger currents. If these higher power tests prove successful, then a full power system will be built. The proposed system uses a pulsed-power supply wizh closed-loop current control during that portion of the pulse when the proton beam is to be bent into Line $D$.

Assembly of the fast-kicker powel supply has begun and the system controls have been checked out. Al1 components are presently on hand except for the charging-supply transformer-recîifier and IVR. Other units are available which will allow low-duty testing pending arrival of the final components. Initial pulsing of the fast-kicker should begin during the next quarter and development is expected to continue for about 12 months.

Work has continued on several types of $\mathrm{min}$ magnets. Nost of the effort has beer. concentrated on installation of electrical bus bars for coil power and water manifolds for magnet cooling. A prototype steering magnet has been fabricated and tested. Production work on the WNR steering magnets is now under way.

\section{Biomed}

The support structure for the "Maxitror 300 " $X$-ray Therapy Unit was modified and adjusted for patient use in the biomed area. This support was moved to the biomed building and installed in an enlarged room prior to the patient-treatment cycle which started in ostober.

A treatment couch from Lovelace Cliric has been modified to be used at LAMPF during treatment runs.

The final design, fabrication, and installation of the moderator-collimator, Model A, was completed and it is now in operation. 
$\underline{\text { EPICS }}$

Alignment measurements were taken on the BM-01 assembly to assist in the alpha-particle tests. An alignment-inspection fixture for the spectrometer magnet pole pieces was designed and is being fabricated. This fixture will be used at the Equipment Test Laboratory (ETL) tooling dock to inspect the contours of the pole pieces.

\section{Other Beam-Line Support}

Two octopoles, a quadrupole, and other beamline components were aligned in Line $C$. Support was given to the task of measuring the fields of the two large $57^{\circ}$ bending magnets in Area C. One of the large High-Resolution Proton Spectrometer (HIRS) magnets was disassembled for repairs and an alignment setup was made to insure proper reassembly of this magnet.

The residual-gas analyzer was used to verify a leak in the $\mathrm{CD}_{2}$ target in Area $\mathrm{B}$. The vacuum piping for the Low-Energy Pion Channel (LEP) extension has been fabricated and installed.

A $32-\mathrm{cm}-0 . \mathrm{d}$. $\times 66-\mathrm{cm}-1$ long vacuum vessel with electrical feedthroughs has been fabricated for use in detector development work.

Inst rumentation

An in-house design of an NMR probe to replace the ones furnished by Cyclotron Corp. has been completed, and several probes are now in production. The addition of a Hall probe to the same body as the NMR unit is under development.

The proton spin-resonance detector for use in the polarized target facility has been constructed and should be completely checked out early in the next quarter.

The $V / F$ converters which are used in the experimental areas were modified to have better linedriving capability. The circuit was successfully tested in the experimental area and used with the CAMAC system.

Electron Prototype Accelerator (EPA)

The machining of the tank-section end segments to provide a new copper-sealing surface for the knife edges was completed. The amount of material removed was between 0.3 and $0.5 \mathrm{~mm}$. The first check of the individual cell frequencies has been made and several cells will have to be retuned prior to further rebuilding operations. Phase $B$ of the rebuilding contract is now complete.

Experiment Support

Experiments 27/176 (replacing Exp. 81) and 124 were mounted in Area $B$. The target chamber and a large bending magnet were aligned. Some hardware and a magnet stand for the 12D24 were designed for use on the spectrometer stand dinghy and fabricated for Exp. 27/176. The following experiment (160) has been planned and a new magnet stand for the $18 \mathrm{D} 40$, which weighs $18300 \mathrm{~kg}$, was designed and is presently being fabricated. Layout drawings and activities lists for these experiments were released.

An aluminum rack for Exp. 142 was welded together for use in the Stopped Muon Channel (SMC). Dust covers for the LEP and the south cave of SMC were fabricated.

During the last quarter, production of the light-tube facility was 34 1ight-pipe assemblies and that of the Multiwire Proportional Counter (MWPC) shop was 64 wire planes and helix helic chambers.

The anticounter support stands and a redesigned target holder were fabricated and installed in support of Exp. 31 .

Some minor changes were made in the target of Exp. 96 and five additional targets were completed. 


\section{II. ACCELERATOR SUPRORT}

\section{1-MHz RF System}

An average of 1857 hv hours was accumulated on each of the 201.25-MHz rf stands during the quarter.

One 7835 triode was lost during the period with a total of $113 ?^{5}$ h of running time on it. The 4664 in module-1 survivec the entire quarter although it exhibited some problems with hv breakdowns across the anode ceramic. Two new 4664s were received from RCA and appear to be good tubes. The two Omni-Wave rebuilt $4616 \mathrm{~s}$ were lost during the quarter; one had over $5000 \mathrm{~h}$ of operating time and the other had only $500 \mathrm{~h}$. The 500-h tube will be run in the test stand to investigate cause of failure.

Three tank windows were lost before the loop cooling was changed to colder water. Modifications to the loop design to improve cooling at the window are being investigated.

Since the accelerator was operating during the entire quarter, very little machine improvement work was accomplished. A prototype controller for module1 drive was constructed but not installed; efforts at improving a troublesome tube socket in the power amplifier modulator were undertaken.

Hardware was ordered and some preliminary measurements were made for equipment to monitor interrank phase on $201.25-\mathrm{MHz}$ tanks and on two or more of the first $805-\mathrm{MHz}$ tanks.

$805-\mathrm{MHz}$ RF System

Routine maintenance, periodic inspections, and preventive maintenance utilized the greater portion of the quarter.

A program to assist the accelerator operators in obtaining maintenance help was written and instituted on the CCR computer.

The testing and evaluation of klystron hv cables were begun and will be completed during the next quarter. The bucket rotator $\mathrm{rf}$ system is now operating into the bucket rotator tank. The klystron crowbar study for this quarter shows a reduction of crowbars on litton tubes (8) from 466 for the 2-month period prior to this quarter to 116 for this quarter. There were approximately 60 crowbars on the 36 Varian tubes during this quarter. Spare rectifier boards and capacitors were ordered for the $805-\mathrm{MHz}$ hv power supp1ies.
The Belden 8871 hv cable under test at the Equipment Test Laboratory (ETL) has $1386 \mathrm{hv}$ hours and 634-h drawing current.

TABLE III - I

805-MHz RF SYSTEMS AVAILABILITY

\begin{tabular}{|c|c|c|c|}
\hline Month & $\begin{array}{r}\text { Downtime } \\
\text { (hours) }\end{array}$ & $\begin{array}{c}\text { Hours in } \\
\text { Month }\end{array}$ & $\begin{array}{l}\text { Avai lable } \\
\text { (percent) }\end{array}$ \\
\hline August & 14.1 & 744 & 98.11 \\
\hline September & 8.4 & 720 & 99.98 \\
\hline October & 10.1 & 744 & 98.66 \\
\hline Quarter & 32.6 & 2208 & 98.5 \\
\hline
\end{tabular}

These downtime hours include: Steering magnet power supplies, quad magnet power supplies, ion pump power supplies for the entire accelerator as well as all the units charged to the $805-\mathrm{MHz}$ rf maintenance section.

Low-Leve1 RF jystem

Construction of the $1-\mathrm{kW}$ amplifier and associated driver stages for use in proton spin-resonance studies has been completed. The amplifier operates at 53 and $106 \mathrm{MHz}$; it is now undergoing final testing.

A preliminary design for a $201-\mathrm{MHz}$ solid-state interface amplifier has been completed and the first unit is under construction. This unit will be tested extensively as part of the new $201-\mathrm{MHz}$ test stand.

Work on the $201 \mathrm{MHz}$ test stand should increase during the next quarter. The power supply pad design is completed and construction is scheduled to begin during the next few weeks.

Total downtime due to low-level rf problems was $5.6 \mathrm{~h}$ out of a total of $2136 \mathrm{~h}$, for an availability of $99.7 \%$.

Power Supplies

The order for twenty-two $800-A, 59-V$ power supplies has reached the prototype stage. A prototype unit will be tested in November 1974.

The seven 150-A, $20-\mathrm{V}$ power supplies for the foil-wound quads and single-tunnel quads have been received. Their "on-off" control bins are being wired.

The 150-A, 80-V power supplies for the tunnel quads are being built; delivery is scheduled for December 1974 . 
The forty-four 1000MCM aluminum cables have been installed in shaft 03 . Installation of the 500MCM aluminum çable from the shaft to the magnet and power supplies is in process.

Work on the magnet current by-pass shunt system is $\sim 50 \%$ complete. The copper plates for the power-dissipating transistors have been received; water-cooling tubes are being installed. The bins with plastic cages which hold the copper plates have been wired and will be installed on the racks soon. The heat sinks and resistors for the power-dissipating collector resistors are on hand. The small reference power supplies for the control loop of the by-pass shunzs have been ordered.

Installation work of new supplies and modifica$t$ ions on many units is almost continuous. A new over-current system with memory, which monitors the SCR currents, the ripple current in the capacitor filters and the dc output current of the power supply, has been designed and prototypes have been made. These units have been installed and are undergoing operational tests. All parts have been ordered to build 120 of these units; they will be installed during the six-month shutdown.

The power supplies on the EPICS line are being made operational.

Mechanical and electrical modifications in at least 12 areas in the basic power supply units are being made as permitted by the operation schedule. Beam Diagnost ics

The activities of the beam diagnostics section during the last quarter have been essentially in three areas: 1) maintenance and repair of exist ing beam diagnostics equipment, 2) organization of maintenance activities and procurement of spare equipment, and 3) testing alld improvenent of systems and s upport of MP-9 machine development activities.

\section{Maintenance}

A large amount of time wils spent on the maintenance of the emjtance syzten. Activities included: repair of driver chassis, actutor water leaks, jaws which were shorted to the beam Iine; adjustone of the short actuator brakes; cleaning and lubrication of actuators; repair and lubrication of a shore actuator ball screw; etc. The solution of samp nor problems in the system produced core relibbic operation imnediately, but some problems, such as the brake on the short linear actuator, will require redesign.

The 495 Hardware Test Program was used to check out the B05 wire scanner system several times during this quarter. Problems with a few of the linear actuator driver modules and wire scanner amplifier modules were detected and the modules were repaired. At the first opportunity, all wire scanner linear actuators will be removed, inspected, lubricated, and measured.

Maintenance of the switchyard and experimental area beam diagnostics equipment has been limited by the lack of sufficient manpower to the repair and calibration of wire scanner amplifiers (integrating type) and driver modules. Because of the number of problems with the amplifiers at the beginning of the quarter, all amplifiers were removed from service, repaired, calibrated, and reinstalled.

\section{Organization}

Steps : 2 re taken during this quarter to initiate better organization of maintenance activities which will enable maintenance personnel to do their jobs quicker and more efficiently. Initial preparations were made for the construction of threc wire cages adjacent to the work areas to be used for storage of spares, tools, drawings, check-out equipment, etc. Spare equipment for some of the beam Jiagnostics systems were ordered. Tool boxes, tools. storage cabinets, etc., kere ordered for the cage areas. The task of wriping mintenance procedures. systet descriptions, and hardware lists was init iated.

\section{nevcloprast}

Tests on the bits mothod far the collecter ind

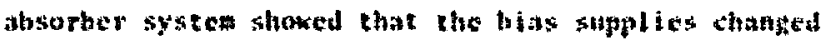

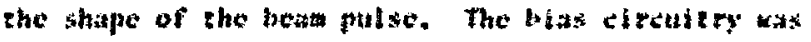
changed and naw has no effect on pulse shapo. test

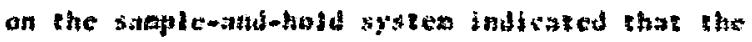

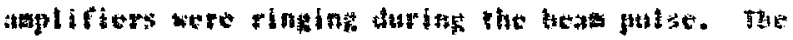

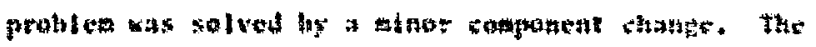

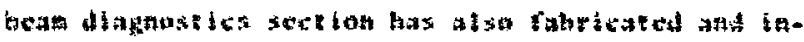

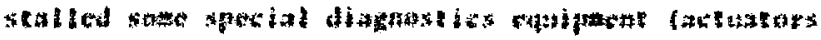

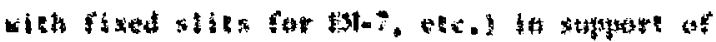

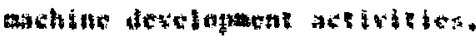


Vacuum Systems

During this report period, nine of the ten $2400-\ell$ ion pumps were replaced with rebuilt pumps. Pump 2-1 is the original pump and will be changed out in the near future. An abnormal amount of accelerator support time has been spent on the 201vacuum due to $\mathrm{rf}$ window failure, consumed ion pumps, and soft vacuum-to-hard vacuum leak。

The transition region (TR) vacuum las been down numerous times in support of the continuing installation and development of equipment in that section of the accelerator. This has been hard on the TR ion pumps and the pumps have had to be replaced twice.

The heating, and subsequent loss of seals at $\mathrm{rf}$ window spacers, has been the main problem with the 805 vacuum during this period.

The switchyard vacuum required relatively little maintenance during this report period. A leaky valve on the rough port of the collimator presented some problens and is being backed with a soft vacuum pump. The normal amount of searching for leaks and plugging holes in the vacuum in Area $A$ and $A$-East was done ard the first eridence of radiation damage to seals and other organics was observed.

the installation and support of experiments has been the min activity in stea B. A turbopump package was installed in the nueleag chanistry cate to facticate the warming up and cooling lown of the liquid seuterius enrget.

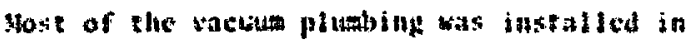
Area fe Auring this period.

Scal desian and develapent are under way to

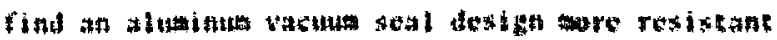
Lo heat.

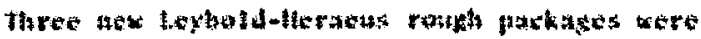
Fecelved and tald reats for ase *
Mechanical Support

The cooling water system for the $201-\mathrm{MHz}$ test stand at ETL is $75 \%$ complete. This test stand water system consists of valving, flow indication and systeri interlocks for the assorted cooling requirements for the amplifier tubes, Fower supplies, modulators, and water loads. Individual flow requirements for the various cooling circuits range from $0.03 \mathrm{l} / \mathrm{s}$ to $9.5 \% / \mathrm{s}$ and add up to a total water requirement of $32 \mathrm{l} / \mathrm{s}$. This system is interconnected to the existing demineralized system. Air pressurization and cooling are also a part of this cooling system.

A Mark II 201.25-MHz tank tuning slug was fabricated and assembled per drawing No. 60Y-1241'3. This unit is presently stored at the ETL as a spare. We have devoted the effort of one man to support of the MP-8 klystron development and work effort. This time has been devoted to fabrication of prototype parts for the klystron gun assembly, 862-A klystron tube.

The design of two 4664 amplifier output cavities was finalized this quarter. Bids for fabrication of this hardware have not yet been received. A cabinet for this amplifjer is being designed. Redesign of the 201.25-311z crowbar resistor enclosure was coispleted and anclasures are being fabricated by the vendor. These enclosures will convert resistor cooling from air to oil.

Support of the heapons leutron Rescarch faciliz: (hxk) thear line magnet and grower supply cooling is cont imuing. 


\section{ACCELERATOR SYSTEMS DEVELOPMENT}

Accelerator Beam Performance

Genera l

The accelerator was operated continuously from the beginning of August to the end of September with $\mathrm{H}^{-}$beams used tor production runs and $\mathrm{H}^{+}$beams used for acceleratior development studies. The main emphasis in the devtlopment work was to study problens involved in high-intensity operation. A series of siort high-intensity runs was carried out early in September using a graphite plug in Line $A$ as a beam dump. A 100- $\mu$ A average beam (2-mA peak current with 400-us pulse width at $120 \mathrm{~Hz}$ ) was run several times for short time intervals ( $30 \mathrm{~s}$ ) with a beam spill acceptable for continuous operation. Tests were also run with 200- $\mu \mathrm{A}$ average beams at higher peak current (4-mA peak), but the best runs still had unacreptable spill at two points in the $805-\mathrm{MHz}$ linac. The transmission through the accelerator on these runs was $>98^{\circ}$, i.e., within the sensitivity of the current monitoring system $(2 \%)$, there was no observable beam loss. No momentum analysis was made during these runs. Wire-scan analysis in the 805-MHz linac wz: subsequently done at low duty and low-intensity halo structures were observed. Efforts are now being directed at understanding and removing these structures.

\section{Injector Beams}

Significant improvements were made in the stability and reliability of the anode power supply in the $\mathrm{H}^{+} \mathrm{C}-\mathrm{W}$ generator. No problems with this generator were encountered in the high-intensity runs after these improvements were made.

Studies were carried out this quarter to determine whether longitudinal acceptance of the $201-\mathrm{MHz}$ linac was affected by operating the beam transport system in a different mode which produces a smaller beam size in this transport line. No significant differences were observed. Studies were also started to probe the longitudinal acceptance of Tank 1 as a function of injector beam energy.

The $\mathrm{H}^{-}$beam chopper was put into operation this auarter and has been used in every production run. Work is still continuing to refine the operation of the chepper and to simplify its tuning.

Matching studies for the $H^{-}$beam line are conwhing and will inciude consideration of the beam size at the chopping plates as well as at the neh prebuncher.

201.25-MHz Linac Beam

During the preliminary alignment work carried out this quarter on Tanks 3 and 4, quad gradients were measured and compared with design gradients; the agreement was good. The measured gradients as well as the transverse misalignments observed for these quad lenses were put into calculations using PARMILA and admittance effects were studied. The results were inconclusive.

Numerical and experimental work continued on Tank 1 and acceptance studies were continued in an effort to finalize the phase scan parameters.

Transition Region (TR)

Considerations of the effects of off-momentum particles on emittance measurements in the TR have been carried out to explain the differences observed at the various emittance stations in this region.

$805-\mathrm{MHz}$ Linac Beam

Investigations continued this quarter on the problem of errors in the lengths of the accelerator structures and possible solutions to compensate for these errors. A rough estimate was made of the portion of the tank length and spacing errors caused by septum-bending during the original structure tuning for Modules 5 through 12. In addition, data from the original tuning were used to make a rough estimate of parameters relating field tilt to septum movement. The parameters so obtained were then used to obtain an estimate of the corrections in tank lengths which might be obtained if these tanks were retuned. It was concluded that these corrections were insufficient.

Numerical simulations were also continued this quarter in hopes of finding a satisfactory solution to the problem caused by the length errors in the 805-MHz tanks. A possible solution is to modify some of the drift lengths underneath the bridge couplers. This could involve as few as 2 or as many as 15 .

\section{Accelerator Systems Development}

Machine Alignment

During this quarter, alignment measurements were made on the interior drift tubes of Tanks 3 and 4 and are shown in Fig. IV-1, based on the 


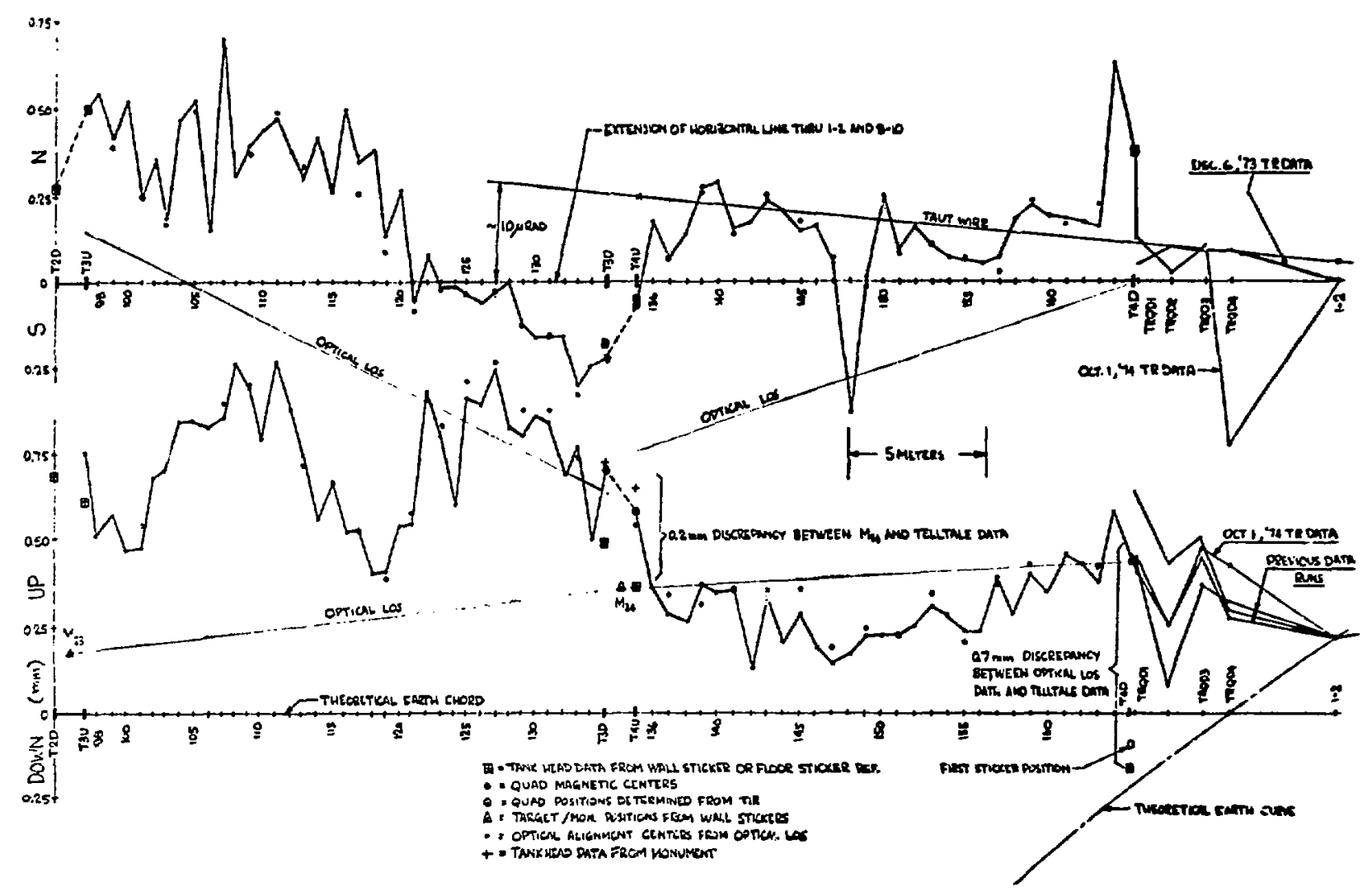

Fig. IV-1. Tanks 3 and 4 drift-tube alignment data.

LAMPF Coordinate System (a 731.5-m earth chord passing through TRQDO4 for vertical, and a straight line between 805 -quad-doublets 5QD01 and $3 Q D 02$ for horizontal). These measurements were made during a single machine-development period and required removing the adjacent heads on Tanks 3 and 4 so a man could enter the tanks and insert plug targets in the drift tubes. Optical lines of sight were established using a monument between Tanks 3 and 4 and targets positioned in beam boxes at the opposite ends of these tanks. These lines of sight were established to be colinear with the average axis of the front end of the 805 linac. The important drifttube positions are those containing quadrupole magnets, shown as small circles in Fig. IV-1. Large deviations exist between the drift-tube positions and the lines of sight. However, since these lines of sight were somewhat arbitrarily chosen, a better evaluation of the alignment is to compare with smooth curves through Tanks 3 and 4 which blend in with the 805. In this case deviations are less severe, $\approx \pm 0.4$ $\mathrm{mm}$. This is still greater than the $\pm 0.1 \mathrm{~mm}$ required for good beam dynamics, and all the drift tubes in the 201 linac will be realigned during the long shutdown.

The decision has been made to establish a permanent monument system based on our knowledge of the present positions of the components of the linac. ${ }^{1}$ Adhesive targets, placed on the rall idjacent to selected quad doublets along the 8051 inac and on the floor $1.016 \mathrm{~m}$ south of the beam lise, will be labeled with their $X Y Z$ coordinates relative to the established LAMPF coordinate system used in previous alignment work. In sector $A$, vertical stickers following the earth's curve will be used and the same horizontal line as used in the 805 linac kill be continued. Future alignment efforts will be simplified by referencing specific components to the rearest wall and floor stickers rather than to adjacent components as has been done in the past. The cstablishment of this system will be completed during the shutdown.

Construction of the electronic circuitry for the LBL pulsed-wire al ignment system has been 
started. This circuit can deliver a $1-k V$ short pulse to a taut wire stretched through the drift tubes. A resulting kick of the wire occurs in a magnetic field which can be used to determine the magnetic center of the drift-tube quads to very good precision. Since the mechanical and magnetic centers of the quads are within $0.1 \mathrm{~mm}$, this method will be used as a consistency check after realignment using optical techniques.

\section{Operations Support Development}

Oeerations/Maintenance History Data Base. During the quarter, the format of the Daily Operation and Maintenance Report has been changed to include section leader risites for all equipment assigned to MP-11 and assignment to specific individuals of selected other units. Adding names to all reports will be completed as soon as definite responsibility can be determined. Blank questionnaires were distributed for obtaining injector equipment informat on and a meeting was held with responsible personnel to insure accuracy and uniformity of responses. The returned information will not be entered into GIRLS, but will be saved for System 2000 .

Several requests for special retrievals of stored data were received. These included availability by systems on a weekly basis from January through September, on a run cycle basis (production or accelerator development) from July through Dctober, for specific locations and selected units for MP-11 section leaders, and for injector equipment

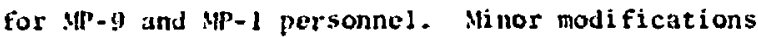
to existing progrims had to be writen for some of she retrievils.

Sytem 2000 Data Base. Preliminary definition of the operation-maintenance data base for System 2000 has beet acconpl ished. A FORTRL program has been written to tanslate key-punched operations and mintenance cards to formats acceptablo to systen 2000.

Conerol tomputer programing and vevelopment Applications Progruns.

The gatin-calibration prouram for the beam current nonitors was wodficd to parmit calibration of any gart of bejm-line and was updated to correspont so the carreme hardware conflguration.

The at cuncug trocedure progetat was ex- tended to automatically set the phase of any specified module. This program has been used routinely by operations personnel to tune the entire $805-\mathrm{MHz}$ linac in about two hours. The next step will be to completely automate the tuning.

The program which reanalyzes the emittance data was changed to read the total current on the collectors with amplitude above a noise threshold, the total current on the collectors with amplitude above an analysis threshold, and the total current on the collectors above an analysis threshold. Total current here means the sum of the current on the 40 collector wires at each of the 100 scan positions.

The 201 phase-scan series of programs was modified to use the calibration data on the phase shifters of the 201 tanks. A calibration scheme for Tank-1 amplitude which will be used by the Tank-1 amplitude-scan program is still to be written.

Accelerator Long-Term Stability Studies. The rf stability study and other studies accumulate such a large amount of data that it is a burden on operations and maintenance personnel to plot and assimilate all the information. A program was written and is now being tested which sifts through the information collected in order to point out the most interesting items, such items then to be subject to further analysis.

A program was written to plot data collected for a stıdy of phase agreement between $r f$ signals from Modules 5 and 6 .

Diagnostic Equipment Develupment Emittancen Measuring (EM) Devices.

An analysis of the EN sample-hold electronics resulted in improvements to certain time constants which eliminated attenuation and ringing problems.

High-power lEM3 jaws were installed and put into service after final alignment machining and bench tests were completed. Initial tests showed that the previous difficulties experienced with inconsistent eaittance data in the $X$ and $Y$ transverse planes were largely eliminuted. No operating difficulty hats been experienced with beam power up to $100 \mathrm{w}$. Instrumentation. The cause of small $\left(5-5^{\circ}\right)$ phase drifts in 
the $\Delta t$ system was found this quarter when it was discovered that small variations in defective filament power supplies for the $r$ amplifiers caused the drifts. The power supplies were failing because of the elevated temperature of the $\Delta t$ racks and ?ack of air circulation. Fans were installed in the racks and on the power supplies to correct the problem. New, more accurate temperature controllers with a smaller control range have been ordered.

New $\Delta t$ loops were installed after Tanks 2 and 3 for continuous phase monitoring. The loop assembly after Tank 2 is a unique design because of the small space available for installation.

A new fast-protect arc detector and counter is being designed for the $\mathrm{H}^{+}$injector.

The electron trap signal amplifier still fails on arcdowns. A tube-type amplifier has been installed and will be used.

\section{Injector Programs}

Ion Sources

$\mathrm{H}^{-}$Ion Sources. The $\mathrm{H}^{-}$ion source has been rin for all production runs this quarter with typically $600-\mu \mathrm{A}$ operation at $5 \%$ duty factor. The only major maintenance on the source has been the replacement of the expansion cup which greatly enlarged and melted when a cooling line failed Tripout of the extractor power supply occurs approximately once a shift and has yet to be resolved.

$\mathrm{H}^{-}$Test Stand. Most of the parts for the beam diagnostic system on the P-11 test stand have been fabricated and installation on the best stand is in progress. Initial beam tests await further work on the source.

Polarized Ion Source. Procurement of various parts of the polarized injector continued this quarter. 'lost of the long lead items for the accelerating column and many of the ion source power supplies have been ordered. The cryopump has been received. Work is still continuing on design of the spin precessor system.

Beam Chopper. The $\mathrm{H}^{-}$beam chopper was put into operation this quarter and has been used to provide several modes of beam shopping. A schematic of the basic system is shown in Fig. IV-2. The chopper deflects the $750-\mathrm{keV} \mathrm{H}^{-}$beam with both

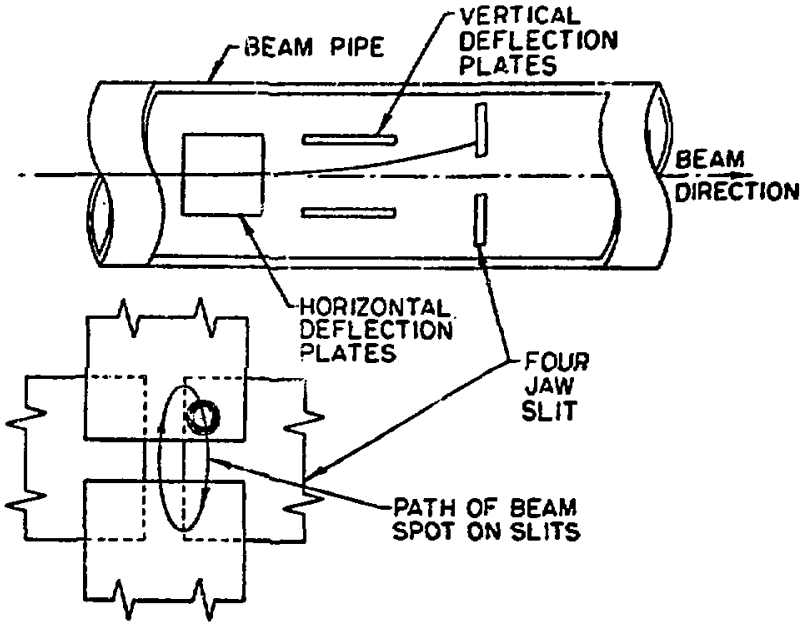

Fig. IV-2. Schematic laycut of $\mathrm{H}^{-}$beam chopper system

vertical and horizontal deflection plates so that the beam follows an elliptical path on an aperture plane having a square hole formed by the EM5 scanning jaws. The chopper is located about halfway between the linac and the accelerating column as shown in Fig. IV-3.

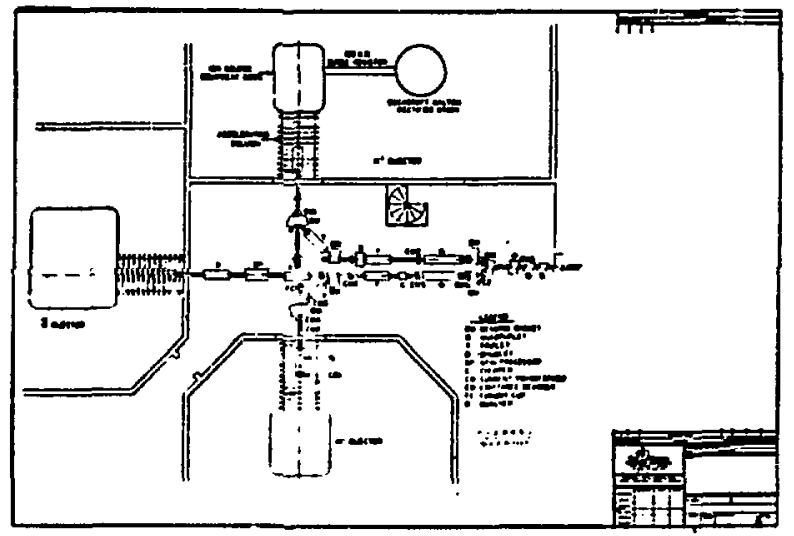

Fig. IY-3. Dual-beam transport system in the injector complex. The choppez is located halfway down the long F.rallel section of the $\mathrm{H}^{-}$beam transport system.

A block diagram of the electronics is shown in Fig. IV-4. The $201.25-\mathrm{M} / \mathrm{z}$ linac frequency is divided by 16 to provide $\sim 12-M / z$ chopping frequency. A variable-width-gate generator is triggered from the timing pulse to contri 1 the pulse width of the $12-\mathrm{M} / 2$ amplifier and of the $0-2 \mathrm{kV}$ pulse generator. The beam-deflecting plates form part of tlse resonant circuit and are tuned by suitable external 


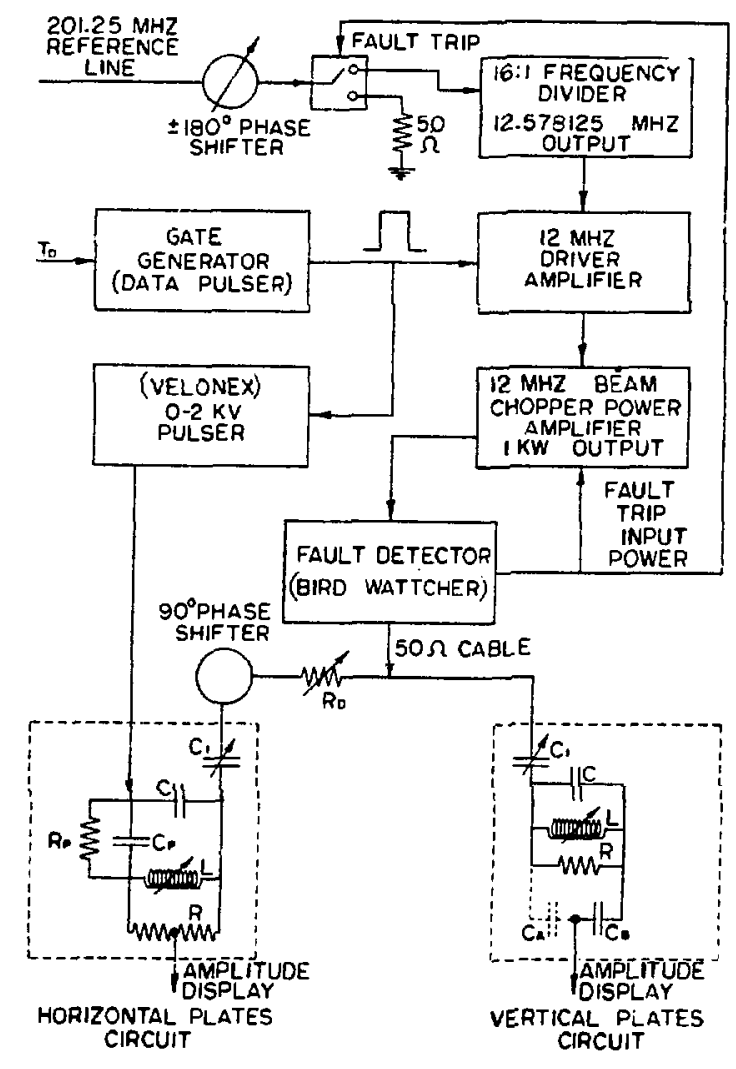

Fig. IV-4, Block diagram of beam chopper electronics.

components mounted just above these plates. When unly the vertical plates are driven by the rf system, there are two beam pulses produced every $12-\mathrm{MHz}$ rf cycle (producing pulses with 40-ns spacing). When both sets of deflection plates are properly driven, the beam is swept in an elliptical path and only one beam pulse is transmitted every $12-\mathrm{MHz}$ rf cycle (producing pulses with $80-n s$ spacing). The $0-2 \mathrm{kV}$ pulser is used to offset the center of this elliptical pattern so the beam goes through the square hole properi;.

The EN5 jaws are ised $t r$, form the square hole for the bean chopping. This choice of aperture plane is convenient since it is then possible to tune the beam for the desired phase-space orientation at this point by using the EMS scanning station. The Jesired beam has a waist of $v 5 \mathrm{~mm}$ vertical $\times 10 \mathrm{~mm}$ horizontal at this point. Drive voltages of $3600-\mathrm{V}$ and $1200 \cdot v$ jeak are required on the vertical and horizonta: plates respectively; the 12-MHz rf system can provide up to twice these amplitudes.

Amplitude stability has not been examined, but appears to be adequate. Phase stability is usually better than $\pm 0.5 \mathrm{~ns}$ per day, but there have occasiona: 1y been large sudden shifts. A closed-loop phasecontrol system is now under consideration to reduce this variation and to simplify the tuneup procedure.

There are four possible operating mudes for this chopper:

1) Single micropulses ( $<5$-ns pulse width) spaced every $\$ 0 \mathrm{~ns}$ or $80 \mathrm{~ns}$ for as large a fraction of the macropulse as desired.

2) Single micropulses ( $<5-n s$ pulse width) spaced every $10 \mu \mathrm{s}$ or more for as large a fraction of the macropulse as desired.

3) Macropulses of 100-ns pulse-width minimum spaced at least $10 \mu \mathrm{s}$ apart or more.

4) Macropulses of adjustable pulse length from 20 to $420 \mu \mathrm{s}$.

Most operation so far has been in the first mode with a chopping length of $50 \mu \mathrm{s}$. The beamtime spectrum in the experimental area has been found to contain $<1 \%$ of the current in the adjacent. 5-ns micropulses. This impurity may be due to the $5 \%$ component of the $\mathrm{H}^{-}$beam arising from the protons in the charge-exchange canal which has $10-\mathrm{keV}$ additional energy. This difference in energy results in a 2.8-ns debunching at the lina. Thus, some of this component wouid be expected to be cap, tured in the preceding micropulse. In order to obtain a cleaner time spectrum, some suppression of this component is required.

To date, the determination of the beam-time spectrum has only been done at the linac exit by the experimenters involved in using the chopped beam. A photodiode with a fast scintillator has been installed in the injector beam line so that direct observation of the chopped beam on a fast oscilloscope is now possible near the entrance to the linac. If such a device could be installed at the exit of the linac, chopper tuneup could be greatly facilitated. The results obtained to date give a FWHM of the chopped beam of $25 \mathrm{~ns}$. iests with a faster scintillator diodc system aie planned in the future to try to determine if this time spread is real or associated with the measurement process. 
Cockcroft-WaIton High-Voltage Generators and

Accelerating Columns

C-W Improvements. Work has continued this quarter to improve the stability and reliability of the $C-W$ generators. A major fault in the installation of the anode power supply on the $\mathrm{H}^{+}$injector was discovered and corrected when it was found that the original $440-V$ power wiring to this power supply was defective. One line of the three-phase power distribution had completely melted and the other two lines were found to be improperly connected. Also, excessive ground currents were present in the neutral line which had been improperly connected in the grounding system. This power supply was retuned and now operates in the same manrer as that on the $\mathrm{H}^{-}$injector.

Another long-standing problem in the operation of the $\mathrm{H}^{+} \mathrm{C}-\mathrm{W}$ generator was found and corrected when tests on the grid-bias supply for the main drive tubes in this generator showed low output voltage which resulted in excessive sensitivity of the anode supply to overload under transient conditions. The cause of intemittent dropout of standby power was also discovered when it was found that an improper substitution of a control relay had been made in this unit. A more modern, solid-state grid-bias supply was built from modular power-pack units and is being tested as a replacement for this original supply.

The series tube in the bouncer failed again after six months. Discussions with the tube manufacturer indicated that short lifetimes had been observed in these tubes under similar pulsed applications and that spark-gap protection might be advantageous in prolonging tube life. A spark gap was installed at the cathode of this tube so as to bypass the high transient currents that result upon arcdown of the equipment dome.

The first hv fault in the $\mathrm{H}^{-} \mathrm{C}-\mathrm{H}$ generator since the installation of the new control system occurred this quarter and was diagnosed and corrected with the new voltage-monitoring system. This system provides independent measurements inside and outside the voltage stabilization loops and permits necessary modifications to be sade to the voltage setpoint during a production run to give the proper operating voltage. The fault was sub- sequently traced to corona on the compensated voltage divider, which was corrected after the production run was completed.

Accelerating Tube Bonding. Tests to eliminate voids in the bonding of accelerating tubes are continuing. The problem of shrinkage and splitting of the sprayed Lexan polycarbonate film as it dries has been solved by using a combination of solvents. Tests on fuld-size ceramic rings can now be started. The bonding of the accelerating tube for the $\mathrm{H}^{+}$injector is expected to be done in December.

Injector Vacuum Systems. The first ion pump to be operated only at low voltage in the $\mathrm{H}^{-}$ dome was replaced; the lifetime of the pumping elements was only six months at these higher gas flow rates. The total throughput of gas pumped was approximately the same as that which resulted in previous failure of similar elements at lower gas-ilow rates. Thus, the total throughput of gas pumps appears to be the important factor in determining lifetime of these elements.

The first beam line pump in the $\mathrm{H}^{-}$transport line failed from hydrogen-induced warping of the pumping elements. Hydrogen elements will be installed in this pump in the future.

High-voltage conditioning of the hydrogen ion pumps in both injectors continues to be useful in extending the operating lifetime of these purms. The capacitance of the 7.5-keV hv capacitor-discharge supply has been reduced from $9 \mathrm{\mu F}$ to $2 \mathrm{\mu F}$ with no decrease in performance.

The contaminant-free-roughing (CFR) system for pumping down the accelerating columns has been modified by replacing the three titanium alloy filaments with two titanium cartridges. This system now has $30 \mathrm{~g}$ of usable titanium in the cartridges as compared to $4.5 \mathrm{~g}$ for the filaments.

Collaborative Programs

Biomed Pion Range Shifter.

Fabrication of the range shifter and $j i b$-boom components is nearing completion. A test stand to support the boom has been designed and is being fabricated. Final assembly of the unit and installation of the necessary services is being included in the Biomed PERT system. Testing will take place at the Biomed facility and is expected to start next quarter. 


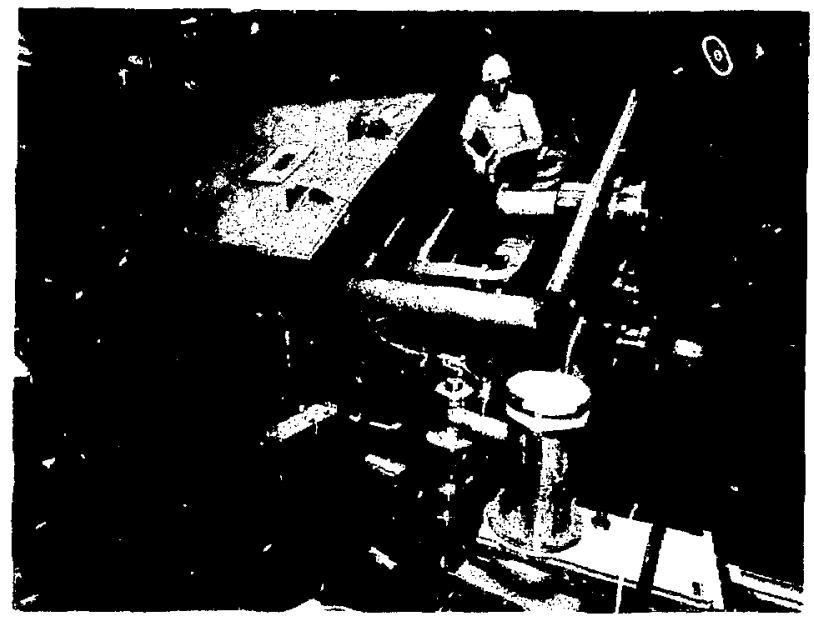

rig. IV-5. Separator system on test stard.

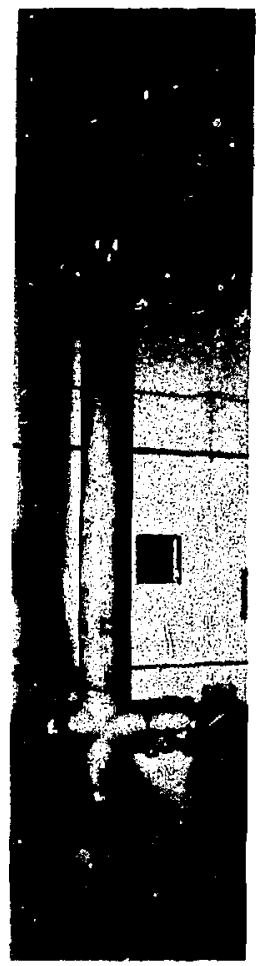

Fig. IV-6. High-voltage line,

\section{EPICS Particle Separator}

During this quarter final assembly of the separator has been completed and the electrodes and equipotential shields have been installed. Photographs of the separator system are shown in Figs. IV-5 through -10 . In Fig. IV -5 the unit is shown on its test stand in Area A without the electric flux box and electrodes, but with the magnet on its carriage attached to the door and rolled partly out of the flux box. The magnet uses a currentsheet field-coil wrap with an aperture of $81 \mathrm{~cm}$. The total weight of the rollaway unit aid flux box when fully assembled is about $12000 \mathrm{~kg}$. The 4.3m high-voltage 1 ine, shown in Fig. IV-6, is designed to carry an internal pressure of $689.5 \mathrm{kPa}$ $\mathrm{SF}_{6}$. It attaches directly to the lower outer feedthrough flange on the door. The electric flux box with the bar electrode cathode and biasing electrode is shown in Fig. IV -7 . The subassembly of the equipotential shields and the vacuum brazed distributed-voltage insulators is snown in Figs. IV-8 and -9 . The shieids are stainless steel; the rod connecting the two insulators connects the proper

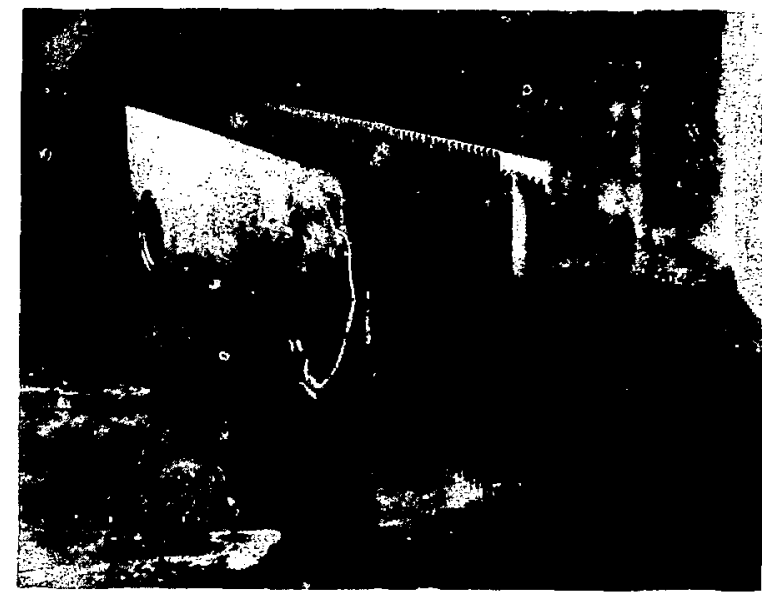

Fig. IV-7. Electric flux box with bar electroy cathode and biasing electrode.

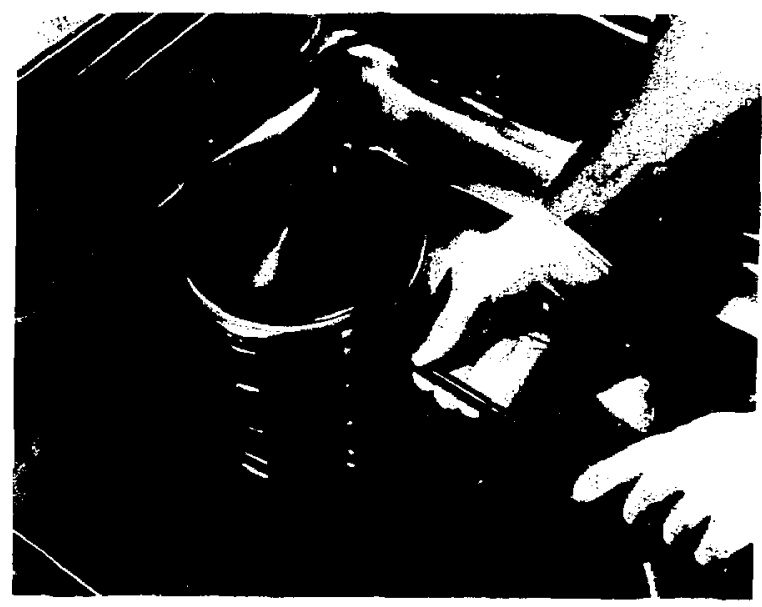

Fig. IV-8. Subassembly of equipotential shields and vacuum-brazed distributed-voltage insulators. 


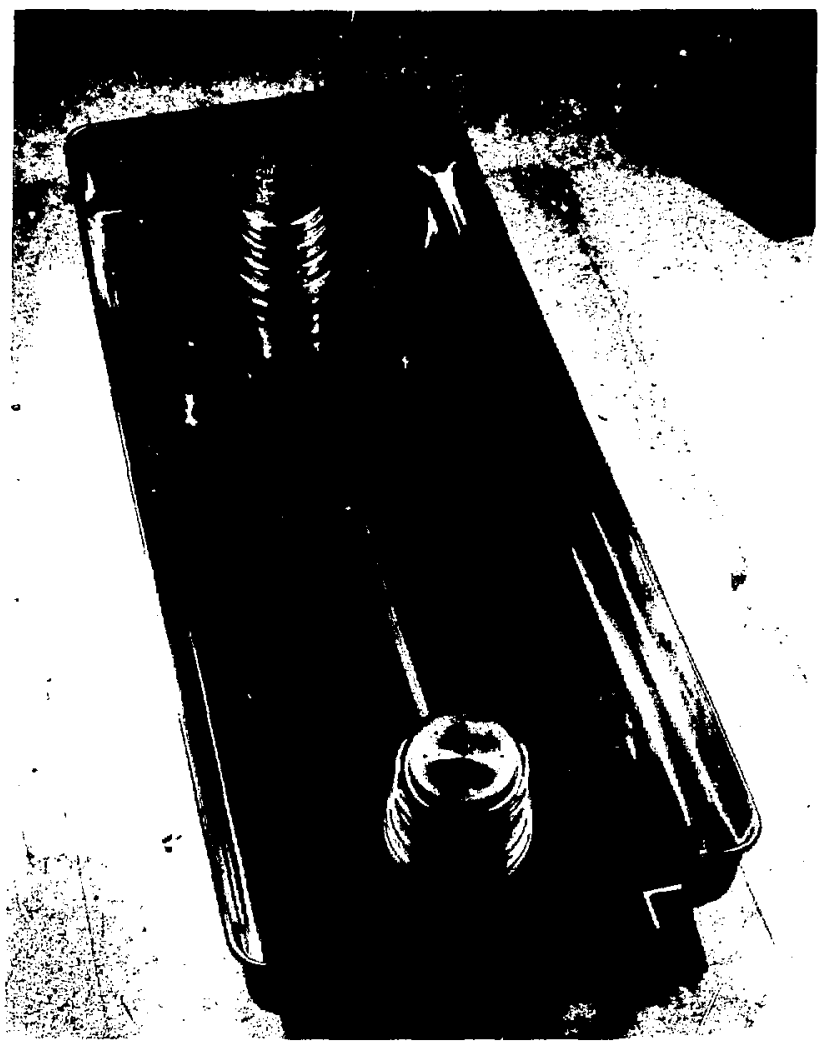

Fig. IV-9. Same as Fig. IV-8, full view.

potential from the voltage divider inside one insulator to the appropriate voltage distribution ring on the other insulator since the shields connect only every second ring. In Fig. IV-10 is shown the electrode assembly with the $320-\mathrm{kV}$ electrode in the center surrounded by the nested equipotential shields. This assembly fits inside the electric flux box. The electrodes lie in the vertical plane and the primary magnetic field component is also vertical.

Another complete set of magnetic field measurements has been made in an at tempt to clear up discrepancies found in the set done last spring. Some differences have been resolved but others stil: persist such as the longitudinal shift of the fiudd peak from the geometric center of the flux box. This shift is $2-3 \mathrm{~cm}$ and is now attributed to the field-coil wrap.

The terminations for the hv flexible cable have bew designed and are being fabricated. Two of the three insulator stacks for the voltage di-

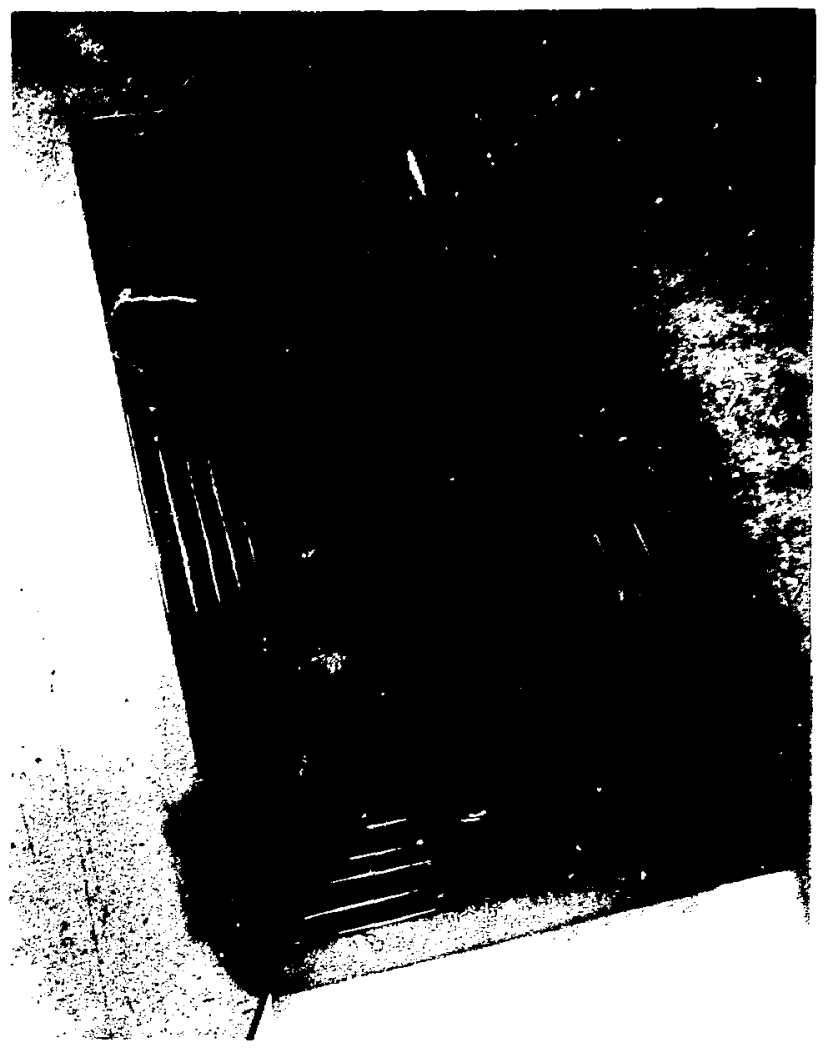

Fig. IV-10. Electrode assembly with $320-\mathrm{kV}$ electrode in center surrounded by nested equipotential shields.

vider have been brazed. All other components of the voltage divider are complete.

The rigid hv line will be shortened to accommodate total horizontal removal of the separator unit with the line attached. Certain other changes were made in the flux-box door seals to minimize maintenance time. Present plans allow for removing the separator for dintenance once a month at which time all radiation-sensitive components can be replaced.

The $\mathrm{C}-\mathrm{W}$ 400-kV power supply has been run up and functions properly. It is expected that this unit will be moved to Area $A$ just before the end of the year and testing of the complete sep rator will begin shortly thereafter.

\section{Reference}

1. D. A. Swenson and D. J. Liska, Los Alamos Scientific Laboratory, personal comunication, October 1. 1974 . 
V. ELECTRONIC INSTRUMENTATION AND COMPUTER SYSTEMS

Accelerator Instrumentation and Control

\section{Injectors}

Work on the $\mathrm{H}^{+}$ion source and $\mathrm{C}-\mathrm{lW}$ hv power supply was largely limited to maintenance and sparecomponent testing. Device spares for both the extractor power supply and 1 ight-link system have been made ready for maintenance use. In an effort to increase the signal-to-noise ratio of measurements in the ion source, a transient current-limiting balun was installed on the input of a compensating feedforward amplifier and a loose mains connection was isolated in the $\mathrm{C}-1$ power source. Tue latter fault has probally caused problems for some time.

$A$ new design of an arc magnet supply was completed for the $\|^{-}$source. This circuit will be installed during the fortheonins shutdown and will provide a better signal-to-noise ratio for monitored ciata. In addition, a joint MP-1/NP-9 etfort towards improved operation of the VAC-ION pumping electronics was completed.

In an effort to provide better Central Control kooin (CCK) operation of the injectors, a program was provided on the control computer to display the status of the injector modulators and to permit an operator to either reset or crowbar the modulators by interacting with the display.

A high-temperature sensor and power shutdown unit has been received from the LASL Electronics livision. This unit will be installed on the Injector Control Room (ICR) NOV/ computer to provide protection for power suppiy overheating--a major problem with Nova computers.

A low-level effort to develop controls for Injector $\mathrm{C}$ (polarized ion source) is being carried out. The transport area controls have been installed for quadrupole magnets, vacuum, and RunPermit. Remote controls for vacuum and magnets have also been installed in ICR. Wiring schedules and some trunkline wiring have been completed. Some dome controls have been ordered, and the dome racks are to be wired in the near future.

Central Control

\section{Control Computer}

The main control computer has, on the whole, rus well during the accelerator run which began in
July. Failures have occurred in the RIU, in disk units, and in some modules of the new memory which was installed during the midsummer shutdown, but no failures have resulted in extended downtime. A cycle counter was built and installed in the computer. It is a counter which is incremented at each computer cycle and can be read under program control. 1t has already received extensive use in timing the execution of various pieces of software. These timing studies are a part of a continuing diagnostic effort which serves to guide both hardware and software efforts to improve response and efficiency of the control system. Design studies, to improve its performance, were begun on modifications to the SEL 840 . Those features which prove to be effeciive and capable of implementation at reasonable cost ill be installed during the long shutdown.

The Priority Interrupt Status Display/Encoder/ Progran Sense Register addition to the control computer is in the final stages of construction and will be installed next quarter. The device will provide system programmers with a more powerful means of interrupr handing and an additional register to aid in software diagnostics.

Control Consoles

The man-machine interface for the operation of the facility is a continually developing system. With improved software, one of the console graphic scopes was reactivated to evaluate its effectiveness as an interactive control device for use by operators. An effort to utilize graphics for control will continue. Development of a more reliable keyboard interfaced through CAMAC as an independent console unit is being carried out. An experimental touchpanel obtained from CERN is being interfaced to the computer through CAMAC. The panel mounts over the face of a TV set displaying programs available for operator usage and selectable by touching the panel.

In order to make developments on the control consoles available to other areas of application, the proposed IEEE/ANSI-IEC interface-bus standard for all future console device hardware will be used. The standard bus, reported on last quarter, will be controlled from a CAMAC module associated with either the IDS computer, in the case of CCR, or a computer system for data acquisition and control, in the case 
of beam-1ine installations. System design will begin next quarter and extend through the year.

A special, hard-wired alarm panel for criticalsystem surveillance (tritium gas, for example) has been installed in CCR. Hard-wired status and alarm devices are considered good practice where personnel sarety is paramount. Information from this system will also be provided to the control computer for use under program control where desired.

Information Display Systems (IDS)

Hardware has been ordered to implement a computer-driven status display for the experimental areas. Information about the current status and operating parameters of the accelerator will be available for $T V$ monitors wherever required. A set of CAMAC modules at the control computer will convert the information to TV signals which will then be routed to monitors in cxperimental areas.

Computer System Software

A system for loading the memories of the remote PDP-11s from the SEL 840 control computer was designed and is being checked. Eventually, each PDP-11 will have a read-only-memory bootstrap which will signal the 840 to transmit its normal software load over the CAMAC data link. The 840 will maintain on its disks a copy of the software for each remote PDP-11. The requirement for access to the control computer from consoles on remote computers at the High-Resolution Proton Spectrometer (HRS) and Weapons Neutron Research Facility (WNR), and the accompanying requirement for programs running in the control computer to be able to operate CAMAC-interfaced devices through these remote computers, have generated a need for a system of message traffic between the control computer and various remote computers. The requirements of these systems have been defined and a scheme of message formats and proucols is at an advanced stage of design. Documentation has been completed for the new graphics language ani for the current data-link software and message protocol.

\section{Accelerator Application Programs}

The fast-protect system display was modified to use the space on the character scopes normally occupied by operator notes in order to guarantee that it can always find space to display fast-protect fault messages. A program which maintains a list of people to cali for emergency help was implemented. A modification was made to the master $t i$ rier and to system softrarc which permits the reading of timed data as much as 75 us before $T_{0}$. Further revisions were made to a series of data-system diagnostic programs to improve their performance and minimize core storage.

\section{Control System Interface Hardware}

This equipment has always provide: an accelerating pulse source to drive stepping motors to full speed to avoid losing a step. Recently, problems arose with stopping high-inertia loads without losing steps. The driver was modified to provide a decelerating pulse source to control the slowdown of these loads without loss of staps.

A minicomputer, teletype, and CAMAC interfacc have been installed in the laboratory for cont inued development of CCR-associated hardware and related accelerator control devices. In addition, a port able 300-character/s paper-tape reader has been set up for use with the several minicomputers used as satellite systems to the control computer. All such minicomputers contain only CAMAC interfaces and no other peripheral:

A new CAMAC data link has been conceptually designed to replace the existing link between the control computer and satallite minicomputers. The new data link will contain a 512-word RAl buffer memory and automatic features for message transfer.

\section{Experimental Areas}

Line A

Software to display the Area $A$ and Area A-East Run-Permit status on a storage scope was completed. Considerable groundwork was done on the wire-scan program in anticipation of the fast wire-scan hardware. The program was modified to permit simultaneous operation from more than one console. Eventually, it is expected to be run from HRS and WNR as well as from CCR. The remote computer interface has been designed and is now being written. A diagnostic and exerciser program was also written for the fast wire-scan hardware. A simple data-acquisition and display program for the new integrating-beam-current monitors was written, and the Line A PDP-11 software was modified to acquire and sum the data. The existing software permits checking out of the hardiare 
while the requirements for a much higher level of software are being worked out among interested grous.

In order to upgrade the existing major Line $A$ instrumentation and provide additionai diagnostics instrumentation for high-intensity operation, a major effort has been initiated in the areas of hardware and computer systems: the wire-scanner profile monitors presently interfaced to the control conputer via RICE data terminals will be moved to a minicomputer and CAMAC-based satellite system coupled to the SEL 840 via fast data links. The system, called the Fast Wire Scanner System (FWSS), wiil speed profile acquisition and overall response to beam-tune changes.

A current-monitor improvement program has been initiated and will ba completed near the end of the fiscal year. Current-monitor outputs are integrated, converted to a pulse burst, and scaled in CAMAC scalers located in satellite computer systems in the experimental area. The information is then sent to CCR via fast data links for use in beam-line tuneup and operations.

Finally, : proposal has been made to provide an input to the Run-Permit interlock chain from the Area A satellite computer based upon fast scans of magnet currents, current monitors, and signals from collimator-like beam collectors. The interlock would be designed to prevent equipment damage due to an off-target, high-intensity beam.

planning and preparation continue toward the shutdown, which will bring Line A up to highintensity operational level. New requirements for operational systems continue to be brought forth. These new systems include the following:

1. Vacuum-to-water interlocks. Several vacuum detectors (now on order) will be used to detect the line pressure near the targets on the beam stop. Upon loss of vacuum, the system will automatically turn off the water to those beam-line components and trip the Run-Permit.

2. Water-cooled shielding overtemp interlocks. Several strings of high-temperature thermal switches will be installed in each area having cooled shielding. These thermal-switch strings and new flow switches will be utilized in a two-of-three logic scheme and used as Run-Permit inputs. Nearly 200 thermal switches are on order for this application.

3. Shielding and beam-stop thermal measurements. As many as 200 copper-constantan thermocouples in a stainless steel/magnesium-oxide rad-hard configuration will be installed at critical locations in the shielding around the targets, on beam-line components, and in or near the $A$ beam stop. The thermocouples are new salvage from the Rower Program and are in-house. However, their installation may be as much as $\$ 20.00$ each. In addition, some readout device will have to be designed, fabricated, installed, and checked out. The readout scheme has not been defined. lilork is in progress on this definition.

Beam-Line Software

The general magnet-setting program was revised to permit on-line entry of solution vectors (expressed in field, current, or voltage) and to reduce the number of character scope lines required by the program. In addition, user documentation for this program was producad and consid rable design work was done on extensions which will enable the progran. to set up beam-line magnets which are under the physical control of remote computers and interfaced via CAMAC systems, either parallel or serial. These extensions will permit the magnets of any secondary beam line, or line $D$, to be controlled by the relatively complex software of the control computer. The switchyard beam spill display was expanded to plot all experimental area beam spill.

Energetic Pion Channel and Spectrometers (EPICS)

A major effort continues toward making the EPICS line and spectromater operational by the end of the shutdown. Preliminary operation and testing of various components has resulted in numerous changes to the control system. ine magnet-pole-face $\left\{\mathrm{H}_{t}\right\}$ windings power supplies have undergone extensive modification, and a number of power supplies have been added. Additional signal and control channels have been assigned to the computer necessitating some reassignment of existing channels, affecting both hardware and software.

The controls system for the scattering chamber target has been designed and drafted. The design of the spectrometer main-frame and curtain-drive controls systems has also been compleited. 
User documentation for the EPICS control software as it presently stands has been issued. A genera] program characterized by considerable operator control and little automatic operation was written to control the EPICS main magnet. Operation of the magnet under this program is expected to provide the information necessary to design a program which will provide support for the automated mapping of the fields of the beam-line and spectrometer magnets.

\section{Test Channe1}

The installation and checkout of the controls for the experimental test channel off of target $A-1$ are proceeding as rapidly as the electricians can complete their work. This will provide experimenters with an inexpensive means for detector checkout prior to full secondary beam-line operation of their experiments.

\section{Operational Secondary Lines}

The Stopped Muon Channel (SMC), High-Energy Pion Channel $\left(P^{3}\right)$, and the Low-Energy Pion Channel (LEP) all require attention during normal experimental operation. The SMC also requires completion of the display and logic system for key relcase to the experimental caves and the beam-plug operating system. Also, the SMC magnet control program was augmented to provide a tuning file, and documentation on the use of the program was completed.

\section{Area A-East}

Area A-East contains the biomedical channel and the radiation damage, isotope production, and the neutrino areas. Design of the controls for the various systems associated with the Radiation Damage and Isotope Production (RADIP) area is essentially complete. The control hardware is being fabricated, and installation should be started late next quarter. Several equipment tests in this area are being supported which might lead to changes in existing designs.

\section{Biomedica.1 Area}

Primary activity in this area was readying the control system for initial patient treatments. A Scriptographics Data Tablet, for use by medical physicists, has been interfaced to the biomed control zomputer. The system provides a digitized $x-y$ coordinate over a large scanning area on demand of the operator. This device will be used in patient treatment and planning.

The basic operating software of the control computer at the biomedical channel was changed as a new release of RSX-11D was received from the manufacturer and installed on the machine. Local enhanrements involving the ability to control the systems from any terminal and to permit different users to use the same operating program simultaneously were installed in the new version. The core storage of this machine was increased to 96000 words, and improvements were made in the subroutine library, file-allocation procedure, and allocation of disk space. Softuare was completed to permit FORTRAi read-and-write statements to apply to the character scope, and the character scope light pen was supported in software, although it still has certain hardware difficulties. Software for the dosimetry instrumentation multiplexer was installed. The beam-line magnet-control program was augmented to provide operator interaction through the character scope. An active task-1ist display was implemented; this display provides considerable insight into the activities of the computer at any given instant and provides a $v j$ sual pattern of activity which can provide reassurance as to the normalcy of operation or an indication of abnormal conditions. Treatment-control software was installed and used in the exposure of the first patients to the pion beam. The software forces the personnel involved in a treatment to proceed in a standard way with input of patient data and treatment parameters. It checks the parameters for consistency and checks the dosimetry setup before permitting target insertion. During the treatment it monitors the treatment and dosimetry setup, logs dosimetry data on a disk storage unit, and maintains a display of dosinetry and treatment information of interest to medical personnel. At the end of the treatment, the $\log$ is printed. While this program was quite useful in the initial treatments, many ways were noted in which it can be improved; it is expected to evolve for some time, as experience is gained in this operation. A channel-status monitor progran was installed. It provides two levels of alarm, depending on how far from the target value the monitored channel has drifted, but it makes no attempt to correct for drifts. A utility progran to provide backup disk 


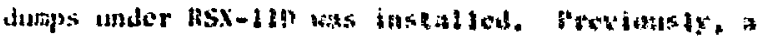

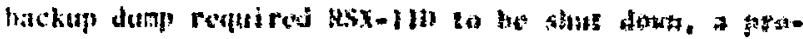

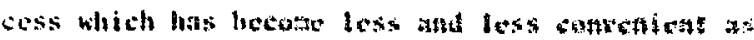
activity on the channel has jocteanth.

\section{Arcis II inn}

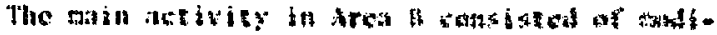

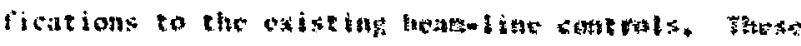

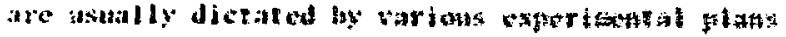

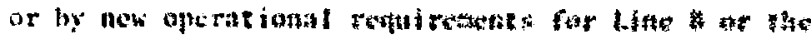
External frotor Beas (1)

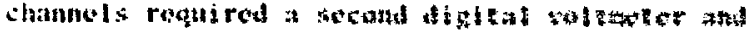
nutiplexer for this iston.

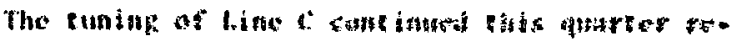

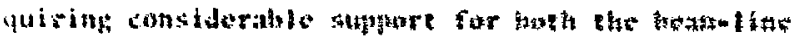

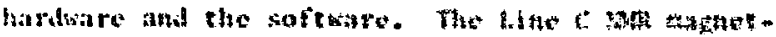

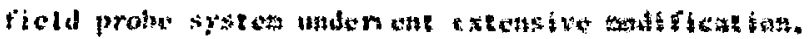

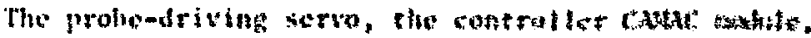

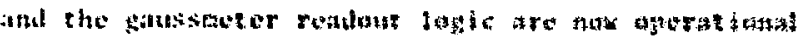

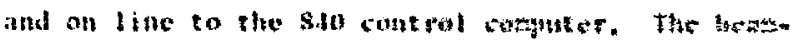

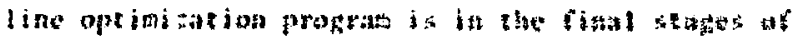

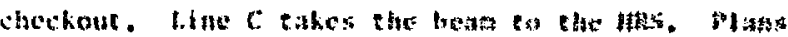
for the developtent of the lats control ransale at?

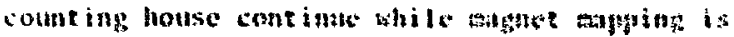
cirried out.

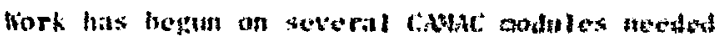
for the IIRS data-acquisition and come rot casmerer (a) Pll-11/15). The lates count ing roon will have a CWh-serviced consolo for horh oxferiantal opera$t$ ions and control of portions of the spectenosetr installation. The lac: interface bus standard beint developed for use with CCR console devices will also find use in HRS. Besign of software for laRs control has begun with the problems of commingation batwen the PDP-11 and the SI: 840 and the general software for the character scope which will be part of the HRS control console.

Support of the IIRS magnet-mapping effort continues in the form of control and data-acquisition hardware maintenance.

Experimental bata Systems

\section{Experimental Area Computers}

The general support of the on-line computers does now, and will always, require a substantial effort. In addition, upgrading of existing systems and development of new ones is a major function. in the software area, user documentation was com-
Ftot

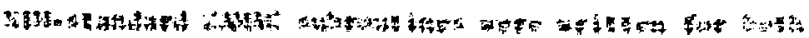

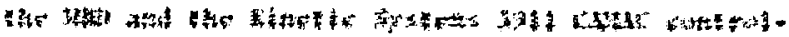

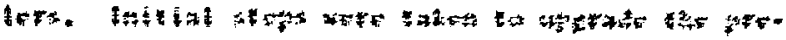

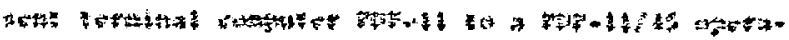

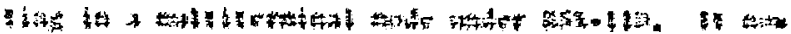

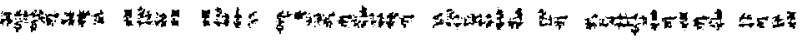

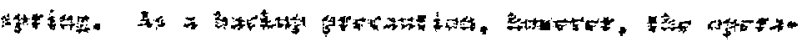

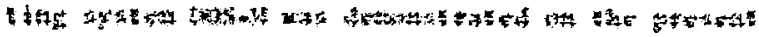

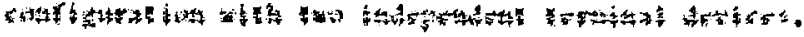

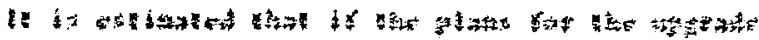

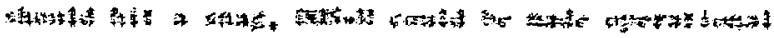

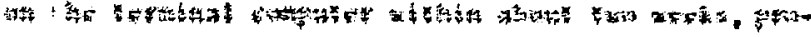

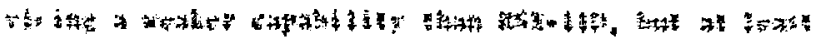

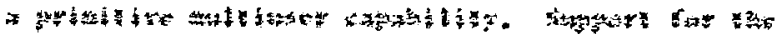

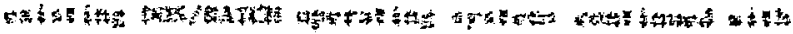

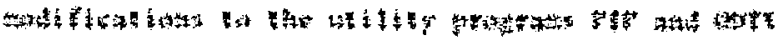

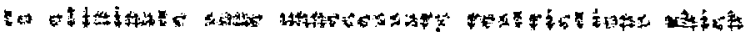

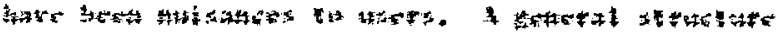

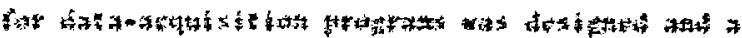

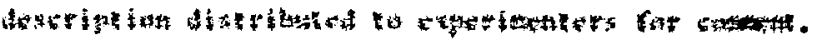

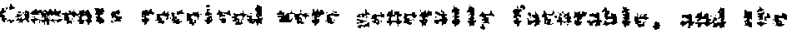

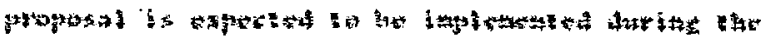

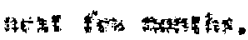

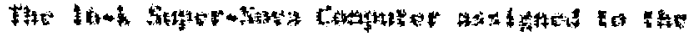

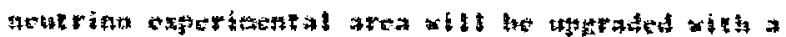

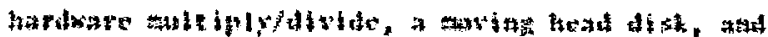

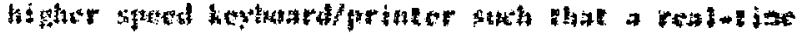

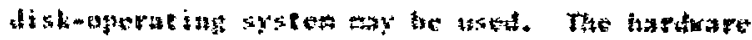

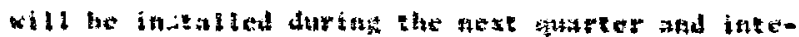

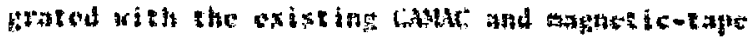
poriptuerals.

\section{Inecofisce llardeare}

Al taw Gate Generators are now in service since the recent uptate and aodification progras. Another user survey til be conducted in the near future to determine if the units neet the varying requi remonts of experimentalists.

An idditional ithpl-des igned MWRC encoder readout module his been fabricated. The nodule is used in acquiring data from up to eight Mxt encoders in a computer- and CMHAC-based experimental setup.

A computer-driven general-purpose cauc module tester has been completed. This unit will be used in testing new and existing modules for both LAMPF Electronics Equipment Pool (LEEP) and beam-line control uses. 


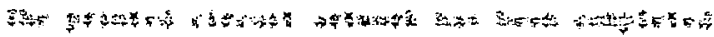

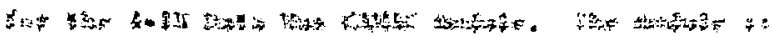

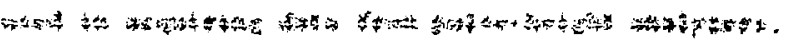

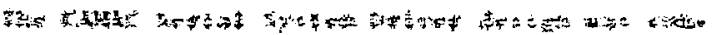

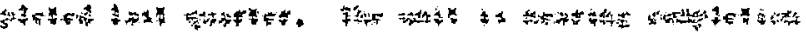

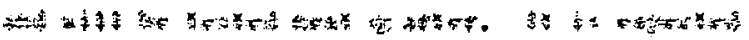

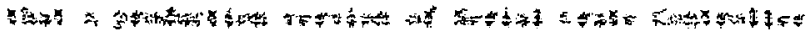

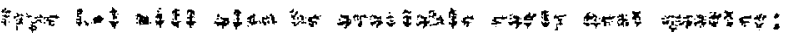

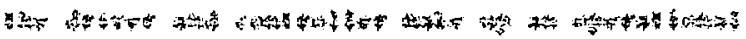

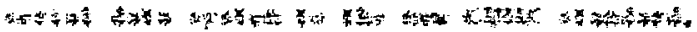

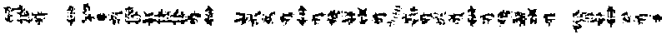

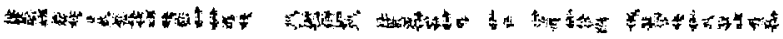

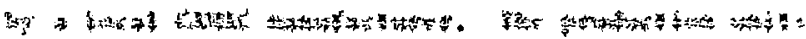

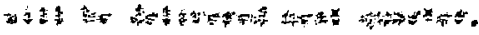

\subsection{8}

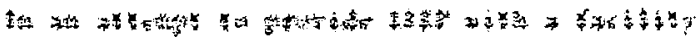

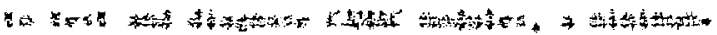

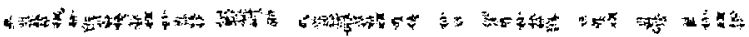

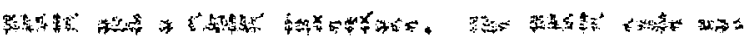

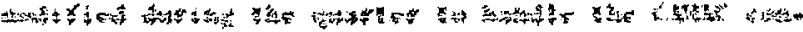

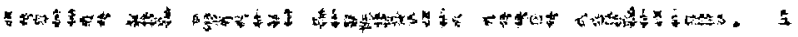

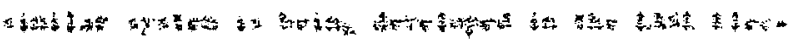

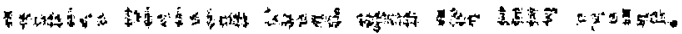

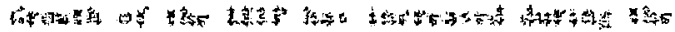

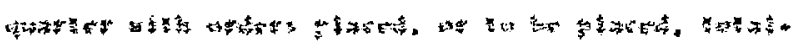

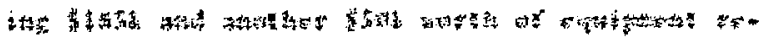

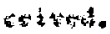

A

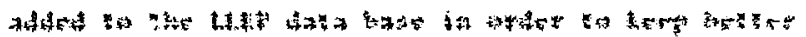

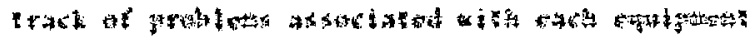

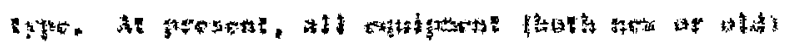

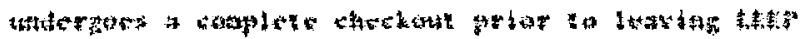

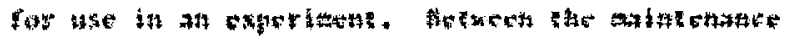

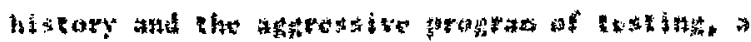

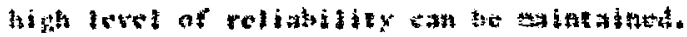

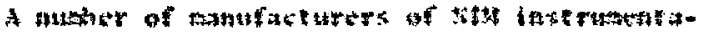
cian trate ine

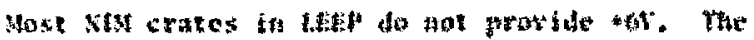

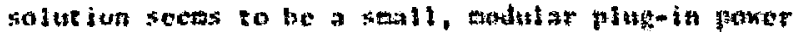

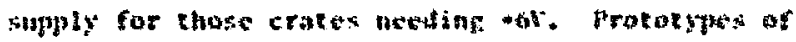

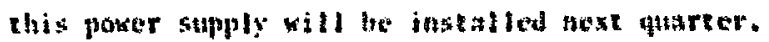

\section{Miscellaneous}

\section{Nuclaar Instrumentation}

In in-house-developed, fast-inserupentat ton amplifier has been packaged four to a sil module with front-panel gain and in/out connections, This

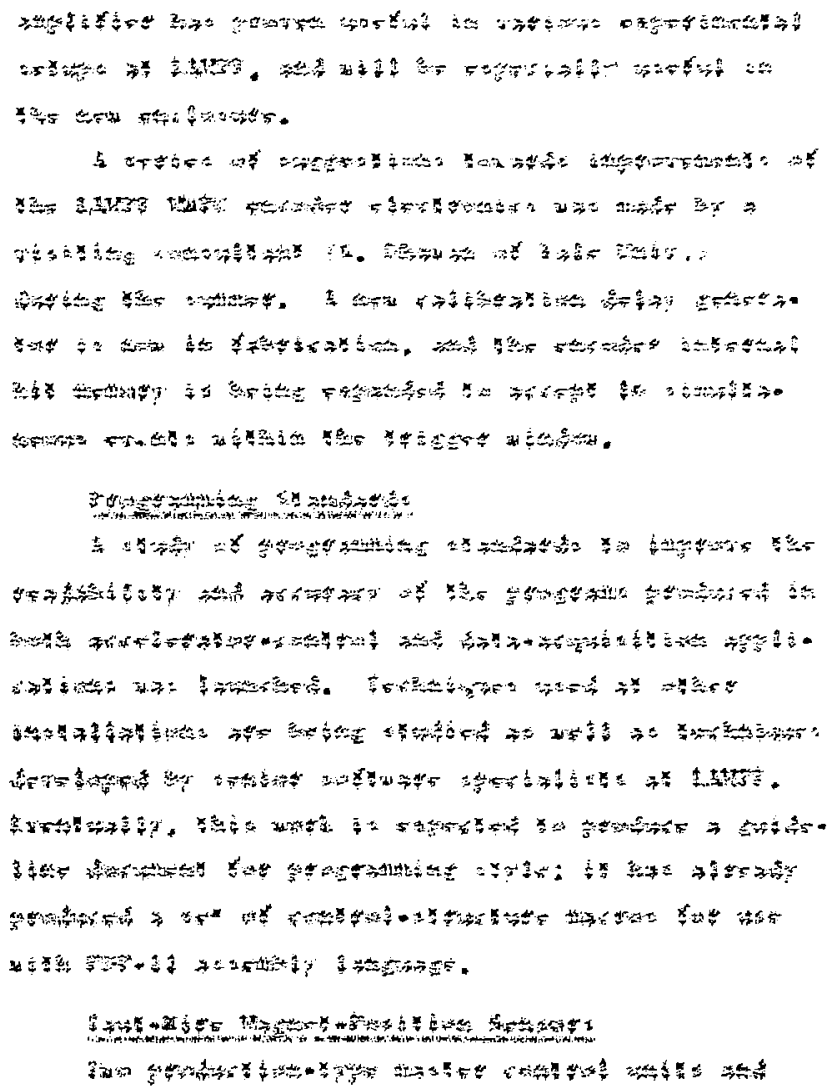

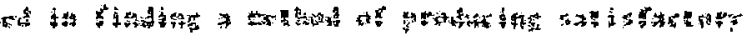

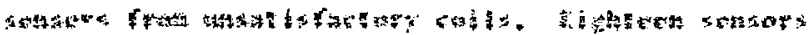

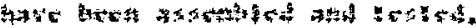

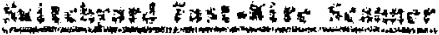

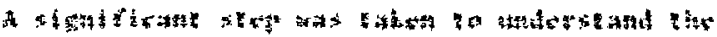

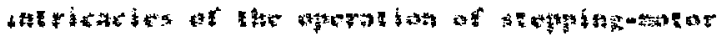

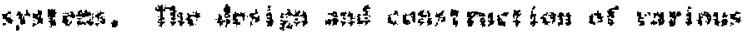

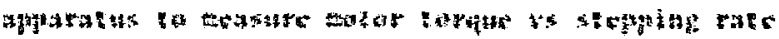

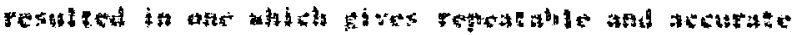

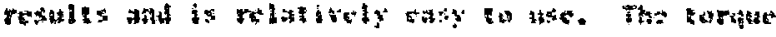

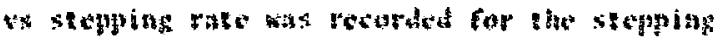

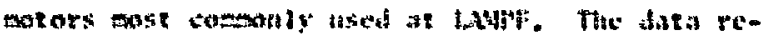

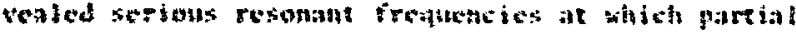

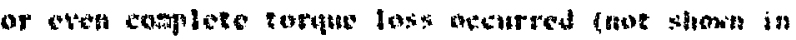

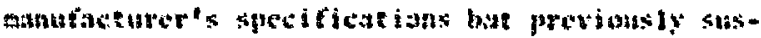
freced). In the process at invextigating Ite 
rcasonance problew, other stepping-wotor mufacturers, weror types, and drive schencs were looked at. Tharouth andiysts of the other wechanical parameters inwolved, combined with she resonance data, has led a Hoter anderseanding of the raming criceria for the sators.

The "Tyyn Il" tunkpolar rosistance-linited swtwer and alternative drive schemes were investiPatch. Hew way of suppressing the transient volt-

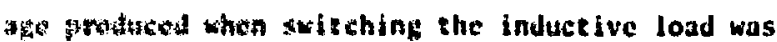
Atof

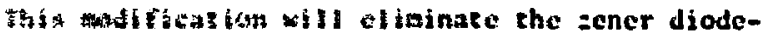

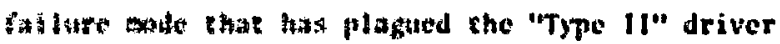
if Ehe nast.
Professional Activities

Several of the technical staff attended professional development and current technology seminars during the quarter. These included courses in logic design, microprocessors, human factors engineering, and data communications. In addition, two members of the staff delivered lect:sres on CAMAC instrumentation and human factors engineering.

Groups from Hahn-Meitner Institute, Berlin, Germany, and CERN, Geneva, Switzerland, visited the facility in a continued interchange of information dealing with computer control and data systems. 


\section{ACCELERATOR OPERATIONS}

General

The accelerator was in operation for the entire quarter. There was a total of 276 shifts, for an average of 21 shifts per week.

As in the previous quarter, the machine was operated in 14-day cycles, each consisting of 12 shifts for accelerator development, 6 for tuning the beam preparatory to research use, and 24 for research. Of the total number of operating shifts, $156(56 \%)$ ware scheduled for research. Because of machine component failures and turn-on and tuning difficulties, only 124 shifts (45\% of total shifts) saw beams of suitable quality for research furposes. Machine availability during the 124 research shifts averaged $70 \%$,

All existing secondary beam lines, the external proton beam (EPB), the Line $B$ nuclear chemistry facility, and the radiation damage and isotope production (RADIP) facility were in use during the quarter. The RADIP rabbit-tube system was empioyed for transferring active target foils to hot cell facilities for the first time. Also, during this quarter the first pion irradiations of tumors on human subjects took place.

Average proton beam currents of $12.5 \mu \mathrm{A}$ at $800 \mathrm{MeV}$ were attained during research runs in the latter part of the period. Proton beams to Area A for experimental use totalled $6800 \mu A-h$, as measured at the A-1 target station, up $177 \%$ from the previous reporting period. An additional $470 \mu \mathrm{A}-\mathrm{h}$ went to Area B. Sixty-six nuclear chemistry target irradiations were performed for Exps. 104, 105, 106, 111, 123,150 , and 161 .

Machine time allocated to accelerator development was used for continued studies of problems of high beam-current operation. Tanks 3 and 4 were opened and alignment of the drift tubes was checked. In the process, a serious corrosion problem on the drift-tube vacuum-seal bellows was discovered.

During a high intensity test run to the switchyard beam stop, the average beam current was increased to $100 \mathrm{\mu A}$ without tripping loss monitors. The test was terminated after $30 \mathrm{~s}$ because of limited heat dissipation capability of the target. The beam current reached $200 \mu \mathrm{A}$ on a subsequent test, but was terminated after a few seconds by spill monitor trips.

The following is an accounting of actual use of machine time during the period:

$\begin{array}{lr}\text { Scheduled operating time } & 2208 \mathrm{~h} \\ \text { Turn-on and tuning } & 244 \mathrm{~h} \\ \text { Accelerator development } & 412 \mathrm{~h} \\ \text { Research } & \frac{878 \mathrm{~h}}{1534 \mathrm{~h}} \\ \text { Total operating time } & \frac{674 \mathrm{~h}}{2208 \mathrm{~h}} \\ \text { Accelerator downtime } & \text { Total }\end{array}$

Machine availability over the period averaged $69^{\circ}$.

TMULE VI-1

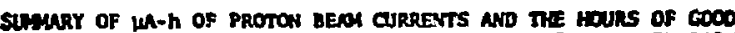
USABLE RESENRCH BEAM TIRE IY EXPERIALATAL STATIOW AND EXPEALIENT MIMBER

\begin{tabular}{|c|c|c|c|c|}
\hline $\begin{array}{l}\text { Experimental } \\
\text { Seation } \\
\end{array}$ & $\begin{array}{c}\text { Experinent } \\
\text { No. } \\
\end{array}$ & $\begin{array}{l}\text { No. of } \\
\text { Shifes }\end{array}$ & Iine (b) & $\begin{array}{c}\text { Intezrated } \\
\text { 2ene Current }(\mu h-h)\end{array}$ \\
\hline \multirow[t]{5}{*}{ LEP } & 25 & $\boldsymbol{H}$ & so & 470 \\
\hline & 67 & 11 & 72 & 635 \\
\hline & 98 & $\mathbf{e s}$ & sol & 4270 \\
\hline & 131 & $\underline{20.3}$ & 123 & $\underline{1130}$ \\
\hline & & 123.5 & 753 & csos \\
\hline \multirow[t]{6}{*}{$\operatorname{sxc}$} & 37 & 73.5 & 457 & 3750 \\
\hline & 100 & 26 & 105 & 235 \\
\hline & 163 & 11 & 57 & 105 \\
\hline & 60 & 10.5 & 63 & 520 \\
\hline & 166 & $\underline{13.5}$ & 83 & 630 \\
\hline & & 124.5 & 765 & 6120 \\
\hline \multirow[t]{6}{*}{$p^{3}$} & 32 & 26 & 154 & 1210 \\
\hline & 34 & 62 & 3183 & 3105 \\
\hline & 67 & 13.5 & 8 & 670 \\
\hline & 99 & 12.5 & 76 & 665 \\
\hline & 123 & 4.5 & 27 & 215 \\
\hline & & 218.5 & 726 & 5265 \\
\hline$\pi A$ & 86 & 35 & 268 & 2055 \\
\hline \multirow[t]{8}{*}{ Bioned } & 243 & $\$ 0.5$ & 490 & 1050 \\
\hline & 44 & 4 & 24 & 160 \\
\hline & 251 & 1 & 6 & 30 \\
\hline & 167 & 11 & 84 & 415 \\
\hline & 84 & 6 & 38 & 90 \\
\hline & 296 & 3 & 16 & es \\
\hline & 195 & 3 & 26 & 205 \\
\hline & & 108.5 & 674 & 1935 \\
\hline \multirow[t]{3}{*}{ EPB } & $\boldsymbol{\prime 2}$ & 67 & 102 & $\cdots$ \\
\hline & 27 & 50.5 & 310 & $\infty$ \\
\hline & & 117.5 & $\overline{712}$ & \\
\hline \multirow[t]{5}{*}{ AB Neution } & 125 & 25 & 160 & 105 \\
\hline & 56 & 32 & 179 & 100 \\
\hline & 189 & 45 & 296 & 210 \\
\hline & 124 & 14 & 요 & 55 \\
\hline & & 116 & 726 & 470 \\
\hline
\end{tabular}


Onc staff tewher fransferred from MP-2 to assume

new duries in the bivision; he will not be replaced.
TaUt: Wh-it

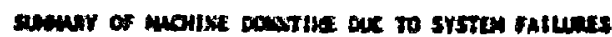

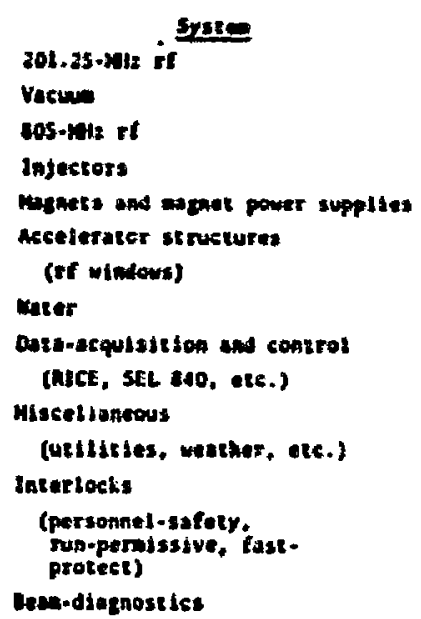

\begin{tabular}{|c|c|}
\hline Deneliest: & $\begin{array}{l}\text { Perceal } \\
\text { of texel }\end{array}$ \\
\hline $3 \times 0$ & A \\
\hline 65 & 10 \\
\hline 1 & $\bullet$ \\
\hline 4 & 7 \\
\hline 42 & 6 \\
\hline 37 & 5 \\
\hline 3 & 4 \\
\hline 2 & 4 \\
\hline 24 & 4 \\
\hline 15 & $z$ \\
\hline 10 & 2 \\
\hline
\end{tabular}

\section{Supuort detivities}

Demand for slosed-circuit celovision-monitoring systens gew rapidly during the period. The demand was met with some equipment found locally; ozher equipment was obtained from Nuclear Rocket Development Site (NRDS) surplus. Monitoring systems were supplied tor Exps. 50, 96, 131, and 14j, The Exp. 50 installation required three cameras.

Personnel-safety systems were installed in Area $\mathcal{C}$ and the new Area $A$ test cave.

Upgrading of primary beam current monitors in the experimental areas is in progress. Toroid pulse signals are converted to pulse trains by means of $V / F$ converters and connected to the control computer system via CAMLC interface. Beam current measurement capability to $>1 \%$ accuracy is expected.

\section{Administrative}

During this quarter, two accelerator operators were promoted to senior accelerator technicians and assigned as operating shift supervisors. Three new accelerator operators reported for work durino the period, bringing the present total to 14 . Four more have been hired and will be reporting during the next few months. Recruiting efforts for two more are in progress and if and when they are successful will fill the complement of twenty.

Two acceiera:or support electronic technicians were hired and two were lost by termination or transfer. We now have three people in this category and are looking for two more for a total of five. 


\section{V11. I:XPERIAIENTML AR:AS}

\section{General}

The emphasis in the experimental areas for the past few months has been to support a fultiple-useresearch program and, at the same tine, to develop che plans and the staff required to achicve the objectives of the long shutdown scheduled to begin in December. During beam-on cycles the low-energy pion chamnel (Li:P), the high-energy pion channel $\left({ }^{5}{ }^{5}\right)$, the stopped tauon channel (SNC), the external proton beam (APB), the neutron area (AB-Neutron), and the bionedical pion channel (Bioned) have been operated with high reliability. In addition, occasjonal use has been made of the tinin target area (TIA), the neutrino area (Neutrino-A), and prototype components of the isotope production an' radiation damage facility (RNDIP). The typical "multiplicity," or number of experiments simultaneously using the beam, has been six, with a peak of nins or ten. By making efficient use of the second cave at LEP, the dual caves at the SNC and the $\mathrm{P}^{\mathbf{3}}$ areas and by taking advantage of floor space available in Area B, as many as 10 physics experiments have been mounted on the floor in Areas $A$ and $B$ at the same time. Approximately 40 experiments have received beam through 31 0ctober 1974, not counting the biomedical program or parasite physics experiments.

Preparations for the shutdown are occupying an increasing fraction of the manpower of the Experimental Areas Group, which has been organized into appropriate sections; the staff level has increased $30 \%$ since the beginning of the fiscal year. In addition, a supplemental design team consisting of borrowed personnel (mostly from the Engineering Department) and numbering about 10 engineers and 10 designers/draftsmen has been assembled to augment our staff. This design team is assigned tasks directly cusnected with the targeting, remote handling, and sholding needed for high-intensity beam operaticn.

Operations

The Experimental Area Manager's Office continued its supervision of maintenance and operation of the major service systems and support of experimental setups. Routine maintenance and minor improvements of the experimental area support systems were carried out. Considerable effort was devoted to improverent of record-kecpinf and reporting procedures. Houtinc liajson with Groups EC-4, Q-26, and numerous experinenters was continued in the genera! support of the experitental program.

\section{Hater Systens}

The process water syechs presently in service have inadequate capaciey for the cooling load required for full operation of the experinental areas. Four more 1.5-19h systems are needed; three of these should be in operation by the summer of 1975 . Sanc modifications have been made to help stretch the present capacity, but any serious mintenance problem results in loss of service until repairs can be made.

The punp in the Xos process cooling water circuit was replaced with a larger capacity pump. The Iddition of this pump made it possible to connect magnets for EPICS and Exps. 57,50 , and 99 to this water system.

The installation of the cross-connect between $\mathrm{XOl}$ and $\mathrm{x} 0 \mathrm{9}$ was completed and the switchyard power supplies are being supplied with cooling water from the 009 system. This modification has made more x01 power supply cooling water avajlable for use in Area A.

In August, major maintenance work on the process cooling water sustems included the removal and reinstallation of both the $\times 03$ and $\times 05$ pumps. Both pumps were rebuilt with new bushings and seals. During September, the pump shaft seal of $\mathrm{x} 05$ developed leaks. The pump was again pulled, disassembled, and cleaned. A careful inspection located small metal chips in the impeller housing that had apparently broken loose from the inside of the casting. After reinstallation and turn-on of this system, excessive vibration indicated further problems. The pump was again removed from the pit, disassembled, and rebuilt. The usual extreme care was taken in reinstallation, and numerous che:ks were made of the vertical alignment before and after turn-on. The X05 system has been running for seven weeks without further problems.

As a result of deterioration of water quality in the secondary loops of the process systems, efforts were made to strengthen the clean-up loops. Resin changes were stepped up and the x04 deionizer loop was activated to operate in parallel with the X06 system. 
Nork is contimuing in the LASL Shops on two new vertical heat exchangers. Several test samples were evaluated to determine the proper cienrance for tubes in the tube sheets. Tubes with 0.2-mm clearance install casily and form leak-tight joints when rolled. These joints were checked wizh a helium leik detector. One sample tuhe, rolled into a short length of pipe sealed on bo:h ends, was themal y cycled many times to $115^{\circ} \mathrm{C}$. This sample did not leak water until tho temperature-cycle was risised co over $150^{\circ} \mathrm{C}$. A laad test on the tubes with $0 . \therefore-m m$ clearance indicated that the tubes would $f_{i}$ il before the rolled joint would leak.

\section{Cryogenics}

The major cryogenies work was the installation, operation, and changing of cryostat systems in the various experimental lines. Operations with the Cryodyne refirigerated liquid hydrogen target for Exp. 32 were completed in carly August and the system was removed from $\mathrm{p}^{3}$-west and installed on the EPB line for Exp. 81 .

This system was operated through the middle of September for Exp. 81, removed from the I:PB 1 ine on 17 September, and installed in $\mathrm{p}^{3}$-west for Exp. 99 by 11 October. Operation is continuing in this 10cation. Support from Q-26 has been received on this cryogenics work.

During October the Area Manager's Office, along with personnel from ll-Division, LAMPF Safety office, ENG-4, and Q-26, was extensively involved in the instaliation and checkout of the Exp. 50 tritium system.

Ma in Beam Lines

\section{Tuning and Operations}

This quarter saw several projects begin to pay off in main beam line operations. of signıficant benefit to experimental area operations was the reduction in typical switchyard start-up time through systematizing tune-up procedures and obtaining better magnet set-points.

Critical tuning locations are found to be the front of Line $A$ and the end $n_{i}^{f}$ Line $X$. With the starting beam carefull; touched up at these locations, an on-axis beam through $L X$ and LB is easily achieved. Figure VII-1 shows $X-B$ wire scans on a good tune.
Reproducibility and systematization were considered part of the task of passing switch.fard tuning over to the Operations Group (AP-2). A manual of switchyard procedures has been compiled and contains, in addition to the tuning procedures, roadmap an! operating hints on the beam line inst rumentation, control program guides, etc. Much of the docunentation on control programs was previously nonexistent. $A$ fallout of this effort was the assignment of a programer to retiew the operator interaction with MACSET.

A checklist for monitoring important operating parameters was developed and serves to familiarize operations personnel with switclyard instrumenta$t$ ion, as well as ensuring proper operation. Use cf the checklist has further relieved the MP-7 beam tuners from time-consuming chores.

Further experience and better beam diagnostics are needed before changes in beam energy can be quickly accommodated in the experimental areas with assurance of a good cune. Considerable fine adjustments were necessary in scaling magnet currents to $647 \mathrm{MeV}$ from good set-points at $800 \mathrm{MeV}$. Pion fluxes observed in four secondary lines at $647 \mathrm{MeV}$ varied in an inconsistent manner from fluxes observed at $800 \mathrm{MeV}$. These difficulties are not unexpected on initial operation at a new energy.

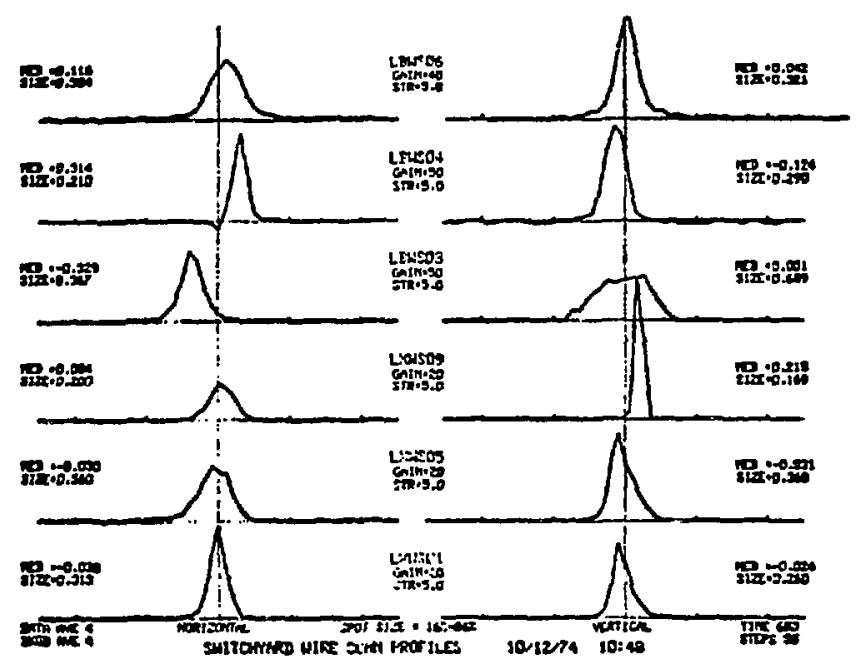

Fig. VII-1. Switchyard wire-scan profiles at critical LX, LB locations during good tune conditions. 


\section{Switchyard}

To achieve and maintair the qualizy of the beam line tunes with less tixe and energy expended, it is necessary to improve the beam-profile monitoring capability. The most cost-effective route in the switchyard appears to be upgrading the existing wire-scanner systen to a higher stroke velocity and data rate; this idea is now a joint NP-1/MP-7 project under the title "Fast Wire Scanner System" and is expected to form the basis for the high-intensity switchyard beam-instrumentation system.

One design problem faced for the planned highintensity operation is detailed knowledge of the beam emittance, particulazly in the outlying tails. Measurements on the existing beam were made with the switchyard wire scanners to provide some input on this operation, as well as to see what level of beam intensity could be measured. The profile measuraments on three wire scanners in a drift space were parameterized as percentile widths (Fig. VII-2) and used in an emittance-calculating program which computed the idealized beam-ellipse parameters (Fig. VII-3) corresponding to the observed projected widths. A plot of emittance vs percentile width indicated near-Gaussian behavior of the outlying beam intensity up to $98^{\circ}$ with rms or $16-84 \%$ emittances normally about $\pi / 30 \mathrm{~cm}-\mathrm{mr}$.

Beam transport studies indicated that a different configuration of strippers and quadrupole magnets in the front of the switchyard would result in improved transmission (larger admittance) into Line $A$ without compromise of Line $x$ and Line $C$ requirements. The new configuration is nearly approximated by a simple retune of the existing $L A-Q T$ magnets, with the interstripper magnets LA-QT-1, $-2,-3$ tuned off and LA-QT-4, $-5,-6$ tuned to low

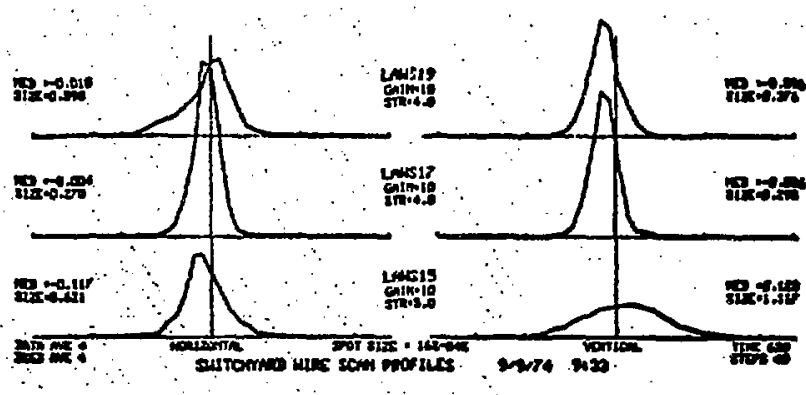

Fig. VII-2. Profiles and computed 16-84\% spot sizes on LA wire scanners for emittance measurement. value for good $L A$ and $L X$ aczeptance; this tune is presently being employed on a trial basis.

\section{Magnet Maintenance}

One of the target cell triplet magnets on the main beam line (downstream of target A-1) developed water leaks during this period. It was replaced by a spare during a no-mal shutdown, and ex mination of the failures is under way. The leaks developed in the nickel ends of ceramic-to-metal seals used as insulators at the ends of coil sections in the magnet, and all the leaks found to date are on the anode (positive) ends of these seals. This and the internal inspection of the seal suggest that the failures are due to electrolytic corrosion enhanced by low water resistivity (as low as $10 \mathrm{k} \Omega-\mathrm{cm}$ on occasion). Further examination of the seals is proceeding to determine what steps can be taken to prevent a recurrence. The water resistivity is now being maintained at $\sim 1 M \Omega-\mathrm{cm}$. Meanwhile, the failed magnet will be repaired. One month after replacement, the radiation fields around the magnet are 10-50 mR/h. During the shutdown, insulators from other beam line magnets will be examined. Most of these magnets are cooled by different water systems and have not been subjected to the very low resistivity water.

\section{Instrumentation}

Coordination of the integrating toroid system for measuring main beam line current has been successful. The preliminary measurements indicate that short-term stability is $>1 \%$ but could be as good as $0.1 \%$.
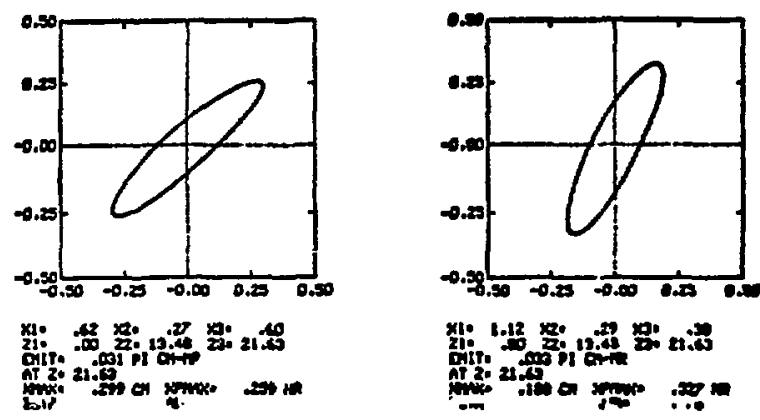

Fig. VII-3. $X$ and $Y$ emittance ellipses and computations for wire-scanner spot sizes in Fig. VI1-2. The ellipse is computed for the location of LA-WS-19. 
Substantial effort has been put into tine development of a nonintercepting centroid monitor for the main beam line. Unlike previous attenpts that were sensitive: to the microstructure of the macropulse, the new device is designed to be sensitive to the envelope of the macropulse. This allows us to use slower, readily available operational amplitiers rather than high-speed tuned circuits. We have bench-tested a prototype and achieved some success. We have wrapped 100 turns of wire around a ferrite block and have simulated the main proton beam using a bare copper wire. By injecting a current pulse into the wire that closely approximates the macropulse, the response and signal-to-noise ratio of our pick-up coil and amplifiers can be tested. Preliminary results indicate that a signal-to-noise ratio of $10 / 1$ for a $10-\mu$ s $r$ ise and a $100-\mu \mathrm{A}$ peak cunrent nuise can be obtained. A prototype unit will be installed in the main beam line to see whether the sensitıity is sufficient.

\section{Heating of beam Line Components}

The MELTEM program, used for calculations of the heating of beam Iine components, has been reactivated, documented, and is now in routine use. To date, calculations have been done for mass slits in $\mathrm{P}^{3}$, LEP, and the beam stop area.

Crude measurements have been made of the heating of a blocl of copper in the A-2 target cell with the present beam. The measurements agree with the calculations to within $30 \%$, which is considered adequate in view of the simplicity of the experiment. Work has started on modifying the program to use it to calculatc activation of components. This probably will not be completed until early 1975.

\section{Secondary Beam Lines}

\section{Stopped Muon Channel (SMC)}

Two remotely operable beam plugs were designed, built, and installed, thus simplifying the personnel-safety system for the SMC. The shielding of the west cave was rearranged aid the gate was enlarged. A helium gas system was built and installed for the whole line. The parasite cave (Cave C) was readied for parasite operation of ix.p. 149. A shield cover was installed over the decay section. in order for the vacuum system has been placed.
Work has started on outlining the jobs which must be done before full beam intensity.

By varying magnet gradients manually, one at a time, the muon flux for a backward tune has been improved by a factor of 4 from the previous best tune. Much of the improvement has occurred in the analyzer portion which had been failing to accept many of the muons at the end of the decay section.

A PDP-11 program (SMCMAG) was developed by M. Kellogg of MP-1 for channel setup which will eventually allow a user to select the momentum beam he neeis and turn the problem of setting the magnets over to the computer. In its present version the program allows direct setting and monitoring of the magnets. Work has also continued on the on-line beam-tuning program (TUNE), originally developed by R. Stambaugh of Yale Univ.

The actual beam-tuning efforts have been directed toward two different classes of experiments, mesic $\mathrm{x}$-ray experiments and muonium experiments.

The current requirements for the first class are maximum $\mu^{-}$intensity in a spot of $100-\mathrm{cm}^{2}$ area or smaller, typically $60 \mathrm{~cm}$ away from the last magnet. Consequently, considerable effort has gone into developing a "high" momentum tune with maximum possible total flux. In particular, Exp. 60 achieved a stopping rate in excess of $40 \mathrm{kHz}$ with a 7$\mu \mathrm{A}$ proton beam. This was in a target area of 50 $\mathrm{cm}^{2}$ and a thickness of $2 \mathrm{~g} / \mathrm{cm}^{2}$. Some effort has also been devoted to developing a forward decay tune which is expected to have 3-5 times as much intensity. To date, the intensity increase has been less than a factor of 2 and the large electron contamination has made this beam unattractive for experiments.

The second class of experiments has rather different requirements. The target (gaseous) is -200 $\mathrm{cm}^{2}$ in area but is located $1.5 \mathrm{~m}$ fion the ist magnet. Because of the nature of the experiment, an extremely clean beam is desired; not only is pion and electron contamination undesirable, but the ratio of muons stopping in the thin gas target $\left\{0.27 \mathrm{~g} / \mathrm{cm}^{2}\right)$ to those stcping in scintillators must be kept as low as possible. This experiment has been running successfully with a $78-\mathrm{MeV} / \mathrm{C}$ muon beam which gives a $\mu^{+}$stopping rate of $3 \mathrm{kHz}$ in the gas target and a range curve $1 \mathrm{~g} / \mathrm{cm}^{2}$ wide. 
In an extended study of SMC characteristics, the limited angular acceptance of the decay section of $\sim 5^{\circ}$ was found to have two consequences: (1) The muon spectrum width is about the same as that of the pions in the decay portion. (2) Because the maximum laboratory decay angles aro much larger than. the acceptance angle for the whole practical rars: of $\pi / \mu$ momenta, the tunes were found to scale (approximately). That is, if a set of quadrupole gradient values at a given $\pi / \mu$ momentum are at a local maximum in the muon flux then the same will be true approximately at a different momentum if the fields are changed in proportion to the momentum. The i... on flux peak is larger at the higher momentum Lase because more particles decay into smaller laboratcry angles.

The present pion collector tune is an achromatic design which was intended to produce a wide pion momentum spectrum width (a FWHM of $8 \%$ has been achieved). By reversing some quadrupoles in the pion collector and reoptimizing the gradients with the SMC optimizer program, a narrow pion (and hence muon) spectrum width was achieved which may be useful for some experiments. This was achieved with no loss in the number of muons per $\mathrm{MeV} / \mathrm{c}$ as compared with the wide tune but, unfortunately, no gain was achieved for this tune.

The analyzer was found to have a wide momentum acceptance (FWHM of $\sim 18 \%$ ) so that the muon momentum width is about the same as the pion width, not just at the end of the decay portion but even at the very end. Because of the limited transverse phase space acceptance, tuning of the channel to produce good phase space at the end of the decay section is very important.

Calculations of the SMC beam are continuing in conjunction with the tuning efforts. Programming efforts have been divided between improving the program and investigating possible tunes. Walter Lysenko, a Visiting Staff Member from Yale Univ., has done the major portion of this work. The empirical tune in use on the channel has been verified in the calculations, but it is still hoped to find a better one by continued searching. A writeup of the calculations is $70 \%$ complete.

As part of the program modifications, the phase space and beam spot plots were improved to produce accurate plots of phase space or beam spot density. An approximate value of the peak muon momentum spectrum value $\left(\mathrm{dN}_{\mu} / \mathrm{dP}_{\mu}\right)$, corresponding to a flat input pion momentum spectrum, is now calculated in the program. Since this approximation involves the use of a single pion momentum value, considerable saving in computer time results.

A new optimizer was developed for determining a set of magnet parameters that maximize either pion or muon flux. This second-order optimizer was found useful for tuning the pion collector portion where muons are not involved. It has not yet been tested on muons because of the large amount of computer time involvec.

Other changes are the addition of a printout of the muon flux at each element in the channel and the addition of an option to start tracing muons beginning with a given element (typically the first element of the decay section).

Low-Energy Pion Channel (LEP)

Only temporary repairs have been made on LEP as the vacuum system, beam plug, collimators, and absorber whee]. all required maintenance. Revisions and permanent repairs will be made during the shutdown. Experiment support mostly involved assistance with preparations for the tritium experiment (Exp. 50).

\section{High-Energy Channel $\left(\mathrm{P}^{3}\right)$}

Repairs were made to adjustable jaws MSO2; the dc cabling was completely redone, upgrading cabling to actual power supply capacity. Experiment 32 was reconfigured in the west cave to the $\pi-\beta$ decay phase of the experiment; after an August run, it was removed and Exp. 99 was installed in its place. Additional experimental shielding was added at various places. A vertical steering magnet and j.ts controls were installed after BMO2 in the $\mathrm{p}^{\mathbf{3}}$ channel.

For approximately $2-3$ shifts per accelerator run cycle, the east leg of the $P^{5}$ beam channel was studied with regard to pion rates as a function of momentum and slit settings. In addition, contamination from electrons, muons, and protons was measured as a function of pion momentum. Conventional TOF and scintillator pulse-height-spectrun methods of particle identification have been employed.

Test Channel

The beam jaws and the beam plug were designed, built, and installed for the test channel. Power supplies (for BMO1, QDO1, QDO2) were connected to 
the magnets and ac power was supplied by the crafts. The control and interlock cabling was pulled to the devices. The instrumentation, controls, and personnel-satety systems were designed, installed, and checked out. Significant amounts of shielding were staihed at the output of the channel. Preparations ar. under way $t$, tune the channel.

Irea $B$ and the External Proton Beam (EPB)

Ixperinent 81 was removed and Exp. 37 was remouned in its place on EPB. Experiment 124 was st' un at two different angles in the Area $B$ neutron racility.

Shiclding was stacked in area $B$ to reduce radiation from hot spots. After a hole was found in the line B beam stop, additienal shielding was placed between the "-BGe trailer and the Line $C$ serivis atise.

in confusetion with the vacuum crew, many improvements wore made to the Line B/EPB vacuum syston:

(a) As satety items, a pressure switch op$2^{2} \mathrm{~m}, \mathrm{ing}$ the fast valve in line $b$ and pressure rupture ?isks were installed in the $10_{2}$ vacuum section of $\operatorname{lin} B$.

(i) A turbomblesular pump was installed in the malear chemistry cave to help overcome continuous problens in the vacuum system near the $\mathrm{L}_{2}$ tariset

(2) Plans nere made to install vacuum ion sausts at fariou; locations in Line B/EPB; one was installed in the nuelear chemistry cave.

(d) A gite valve was installed which isolister the Faradia sup at the end of EPB. This will allovite punpowion problems when the vacuum system for fipk is let up to air.

\section{Viagnost ic duparatus for Secondary Beams}

A prototipe system of two helical chambers has bere tested and used as a means for secondary watin lint tunctip. The computer programs have been witich and fested on a PDP-11/45 computer. The system was put in the second cave of the EPB line, where scattered protons from a thin target were obsered. The proyram took the coordinates from each chantior and cisculated the target coordinates, scattering angles, and $x$ and $y$ intercepts. All far rimeters were histogramed and any two parameters sould be displayed on a storage scope in the form of a two-dimensional dot-plot. The program was successful, and the beam spot could be determined at counting rates up to $500 \mathrm{kHz}$ (instantaneous). However, modifications had to be made to the program for use on the PDP-11/20 computer. In so doing, some software problems developed and prevented any meaningful testing. Work will continue on the system.

Magnet Measurement

Measurements have continued on the magnets for the Weapons Neutron Research facility (Line D). Since April, fourteen P6III58/4, one H4II12/4, two P7II68/5, and five $3 Q 12 / 4$ bending magnets, and the five tape-wound $2 Q 6 / 6$ quadrupoles have been measured. A $12 Q E 18 / 12$ quadrupole was remeasured with chamfered pole ends.

Magnet-measuring service to experimenters also continues. Equipment has been moved to Area $C$ for measurements on the large-bore octupole magnets, which are measured in both steering magnet and quadrupole configurations. The EPICS separator magnet was remeasured after its move to the experimental area. The 30040 magnet was mapped in its $C$-configuration.

Standardization of current measuring elements has continued, especially in line $C$, using the Guideline 9940. A check of the regulator stability on one of the EPICS power supplies was also made with this instrument.

Polarized Proton Target Development

Modification of the prototype cryostat is now essentially complete; material procurement and fabrication caused some delay. The second test cycle will begin soon.

Arrangements for the loan of a magnet from ANL have been made. Fabrication of a cryostat for the split-solenoid superconducting magnet is proceeding. The construction of the NMR detection system has been delayed due to a shortage of manpower.

Radiation Effects and Isotope Production Facility (RADIP)

Progress in the construction of this facility is now more apparent. The stringer housings have been realigned, and trial installation $0:$ the stringer-drive gear boxes has been made. The controls platform and the ra:ks for the control 
electronics are in place. The clutch assomblies for 12 stringers are assembled, and remote tooling to handle the first targets has been fabricated. The viewing periscope and the target-transfer cart (to move radioactive targets to the shipping flask) are ready.

The first two dosimetry packages were irradiated in the RADIP cavity and several aluminum foils have been inserted in the proton beam using a stringer to check the proton-bean instrumentation. Good agreement exists between beam profilcs and autoradiographs of the foils. Autoradiography of the foils can be accomplished on-site, giving a check on the harp beam position and profile, and on the alignment. The first neutron dosimetry indicates a neutron flux at the $R-10$ stringer position of $2 \times 10^{13} \mathrm{n} / \mathrm{cm}^{2}-\mathrm{s}$ for $1 \mathrm{~mA}$ of $800 \mathrm{MeV}$ protons incident on the beam stop.

Immediate requirements for this facility by users are now shifting from completion of the full capability to a demonstration, with restricted capability. Since the usefulness of the facility is obviously tied to LAMPF beam intensity, it is proposed to demonstrate isotope production and handling ability before the shutdown, and to continue dosimetry and spectrum studies in the RADIP cavity. Essentially all of the hardware for this is on hand, but improvements are needed in the stringer design. Eventually, the stringers will have to be reworked or replaced. A water cooling system will be required; solenoid control valves for the first three stringers are on hand for this purpose.

Remote-Handling and Targeting Systems

\section{Target Cell A-1}

The final desigr work of the target box, collimator, and connections to LEP and EPICS is in progress. Additional study is under way on the joint between the front and rear vacuum chambers, both for target cells A-1 and A-2. A mockup of the EPICS window cone is being made. Vacuum and heat-conduction tests will be performed on the mockup to verify the design prior to manufacturing the final window parts.

Fabrication of the A-1 target mechanism is nearly complete. The shielded drive-system design for the A-1 target is complete and ready to be sent out for bid. The shielding plug which permits the removal of the A-1 target mechanism and the EPICS separator box is being designed. The in-cell shielding design has also started.

Lucite covers for the target box and the profile monitors were replaced with stainless steel plates.

\section{Target Cell A-2}

The collimator for line $A$ and the heat shields for both $\mathrm{P}^{3}$ and $S M C$ heve been worked out in concept with acceptable material temperatures. A design review will be held in a few days on the A-2 cell concept. Conceptual designs have been completed on the access system for the target box and the removal casks for the target and target mechanism. The detailed designs of both the A-2 target mechanisms and the shielded drive system are nearly ready to be released for bid.

Lucite covers for the target mechanism and profile monit:r were replaced with stainless steel plates.

Area 1 -East

The work to be accomplished on target cell A-5 (Biomed) was PERTed; the actual design has been started, with the addition of an engineer assigned to this project.

A new hydraulic pump was installed in the Biomed target system. A complete pump package was also installed as a back-up unit, but is not yet operational.

The conceptual design of the neutrino shutter at the main beam stop $(A-6)$ is complete, and the detailed design has started. Conceptual designs for the beam stop translation mechanism and the harp and current-monitor vacuum boxes have been completed. Calculations on the beam stop are in progress.

The water-cooled copper beam stop sprung an irreparable leak. A temporary unit with 10-kw coo:ing capacity was designed, fabricated, and installed.

\section{Tooling}

The conceptual design of several universal tools for remote handling of main beam line components by Merrimac has been complezed. Detail 
designs will start soon. The designs of the $1 \mathrm{ift}$ fixtures for the Line A quadrupoles and the LEP first doublet have been completed. Designs are in progress on the remote-handling fixtures for additional secondary line magnets.

\section{Merrimac}

The work necessary to make Merrimac operational is proceeding well, although most of the effort to date has been a revicw of and familiarization with the various systems as they stand. Two ball lead screws that lift the box were damaged in operation and replacements have been ordered. A complete wiring diagram is being generated by tracing the existing wiring. Additional pass-throughs to assist in magnet handling have been designed and the fabrication has started. A qualified technician has been hired to be in charge of Merrimac.

Hot Cells

Vour hot-cell window-box assemblies were completed, filled with ferro-phosphorous ore, and set in place. Manipulators were installed and the north hot cell now has a limited-use capability.

light CRL Master/Slave Manipulators were acquired from the New Brunswick AEC Laboratory.

Six Master/Slave Manipulators from the NRIS $K-M L D$ Building were decontaminated and completely overhauled. The manipulator hoist system was installed in the hot-call arca. A qualified technician has been inired to supervise work in the hotcell area.

Shiclding

The inajor shiclding effort in the experimental areas was to close off weak areas in the temporary shielding around the target stati. ? covers to decrease the general background on the experimental floor. The weak areas are located in Area $A$ where the in-cell shielding and Merrimac doors covering the cells have not been installed. The improved shiclding allows routine operation at $10-\mu \mathrm{A}$ average current.

Design work continued on the Merrimac shield doors for Targets $A-1$ and $A-2$. Most of this has been completed for $A-2$, and a major redesign of $A-1$ doors was accomplished to eliminate special concrete battery plates. These plates turned out to be cxtremely expensive with too long a lead time for fabrication. Sufficient design work has now been completed to start the fabrication of the steel door boxes.

Parallel design efforts have been started on the canyon-fill shielding for the secondary beam lines and for the in-cell fill around the Line $A$ targets. Engineers and designers from the LASL Engineering Department are assisting in this work in order to complete desig: and start fabrication before the shutdown.

Installation work in Area A during the past quarter included replacement of the shield cover of A-2 target box. Part of the A-1 cover was removed to permit replacement of the main beam line triplet. Some temporary shielding was added near the front of the SMC. A concrete block cover was installed above the first 10 magnets of this beam line.

At $A-1$, the shield cover above the upper part of LEP was removed for hardware modifications. Additional shielding was installed in the LEP canyon and between the line and the LEP counting house. Additional concrete shielding was placed on the EPICS face of the A-1 shield.

Because of gamma activity in the process water systems, concrete shielding was placed in front of the exposed valve caves. Permanent covers have been designed for these caves, incorporating the remote valve actuators and instrument-line penetrations. These will be installed during the shutdown.

Numerous changes have been mad 3 in the experimental caves at the ends of the secondary beam channels. Cave walls and entrance mazes were built for Exp. 50 on LEP and for the test channel. Both the east and west caves for the SMC were modified. The east cave of $p^{3}$ was extensively modified and enlarged to accommodate Exp. 34, and the west cave was adjusted for Exp. 99.

In Area A-East, Targct Stations A-5 and A-6 were opened for hardware modification. No change was made in the shielding configuration. During the shutdown, additional shielding will be installed at $\Lambda-6$ and around the A-5 target box.

Majos changes in the shielding in Area $B$ were made on the EPB line. The shield for Exp. 26 was removed and the EPB beam stop was modified to incorporate the Faraday cup. A concrete roof was placed above the upstream section of the EPB line 
to reduce the background in the Area $B$ service building. Shielding was placed alongside the P-DOR trailer to provide additional protection from the Line $B$ beam stop.

Major installation work in Line $C$ beam stop has been completed.

All scrap shielding is being collected and an inventory is being made; procurement of new material is under way. A contract has been let for concrete blocks and delivery has started. An order for $10^{6}$ $\mathrm{kg}$ of steel slabs was placed with U. S. Steel at the high price of $\$ 230 / 1000 \mathrm{~kg}$. Most of this material has now been delivered to Los Alamos and will soon be fabricated into sections of the large Merrimac doors.

Switchyard Improvement Program

A switchyard improvement program is under way with the objective of ensuring that the switchyard will transmit a $1-\mathrm{mA}$ beam (100 times the present beam) while keeping the bean spills to present levels. At $1 \mathrm{~mA}$ the maximum tolerable spill is $100 \mathrm{nA}$. The program, which began in August, consists of:

1) Optics calculations and design studies; 2) beam measurements and beam experiments; and 3) beam diagnostic instrumentation development. Some progress has been made in each area.

\section{Optics Calculations and Design Studies}

These studies are guided by two major criteria: to increase and optimize the acceptance of line $A$, and to decouple Lines $A$ and $X$ as much as possible. The surest way to minimize spill problems is to have as much acceptance as possible. Decoupling of Lines $A$ and $X$ will simplify control and tuning and allow more flexibility in utilizing the areas served by each.

Early in the studies it was realized that the acceptance of Line $A$ could be doubled by retuning the line A triylets. One weakly focusing triplet or doublet is sufficient. These tunes are accompanied by a reduced sensitivity of spot sizes and centroids to small percentage changes in quadrupole currents. These tunes are better matched to Line D than the previous design. They are also well matched to Line $x$ requirements. The optics of phase-space tailoring for line $C$ are changed but still meet the Line $C$ requirements.
For some time we have realized that it is difficult to meet Line $\mathrm{C}$ requirements at $\mathrm{LX}-\mathrm{ST}-02$ because only a doublet is available for matching Line $X$ to Line $C$ at this point. Transport studies show that four quadrupoles are needed for optimal matching.

Other studies are under way or are planned. These include new layouts for beam control and beamdiagnostic instrumentation, acceptance calculations for Line $X$ asa Line $C$, and calculations of aperture losses due to misalignment and steering errors.

Beam Measurement ar.d Beam Experiments

A program of measurements and experiments has been planned to answer the following questions about the beam:

1. What transverse phase space contains all but $10^{-4}$ of the beam?

2. What is the momentum spectrum of the beam emerging from the linac? Once again, one is interested in the "tails" that are $10^{-4}$ of the total beam.

3. How accurately can TRANSPORT describe the real transport of beams in the switchyard?

4. How much beam is presently spilled in the switchyard?

Careful work with switchyard wire scanners yields information on transverse emittance. Computer codes are being assembled to fit the wire-scanner data so that the distribution in transverse phase space can be estimated. Repeated measurements of spot sizes indicate an rms emittance of $0.03 \pi \mathrm{mr}-\mathrm{cm}$ for both $x$ and $y$. Assuming that it is Gaussian means that only $66^{\circ}$ of the beam lies inside a $\pi / 10-$ mr-cm ellipse in $\left(x, x^{\prime}\right)$ and $\left(y, y^{\prime}\right)$ phase space. This suggests a great deal of beam must be stripped away to meet Line $C$ requirements.

A study of beam halo utilizing a movable scattering target is planned. Spil1 monitors will measure the amount of scattered beam. Calibration would be achieved by rloving the scattering target sufficiently into the main probe where the loss could be measured by current monitors. We hope for a sensitivity of $10^{-4}$ of the main beam.

Time-of-flight measurements are under way to study the momentum spectra of proton beam in Arca $A$ and the switchyard. These studies utilize a portion 
of the beam which is chopped and has either 40 or 80 ns between micropulses. In Area $A$ the time of arrival is marked by the appearance of an electron in a secondary beam line and time is measured with respect to 201-MHz rf signal which is counted down by a factor of 16 . The dogleg in the switchyard passes a beam with $\Delta P / P=5$ to $10 \%$; the rest is lost. Time-of-flight data from the $p^{3}$ channel, in a recent run, showed 0.5 to $1 \%$ of the bean in a 5ns "shadow" due to incomplete chopping. "Only about $2 \times 10^{-4}$ of the bean occurred beyond that in TOF. Translated to $\Delta \mathrm{P} / \mathrm{P}, 0.02 \%$ of the beam had $1.7 \%<$ $\Delta P / P<-7 \%$. Steps are being taken to reduce the anount of incomplete chopping. Another TOF experiment is being planned for the switchyard to look at the arrival time of protons before the first bending magnet. Here there will be no cut off due to the momentum pass band of the deflecting system.

Beam-Diagnostic Instrumentation Development

A coordinated effort between several MP-Division troups has been started for instrumentation development for all the proton beam lines, not just the switchyard, Developments are being initiated to rad-harden Area $\Lambda$ harps and toroids, to improve the accuracy of current monitors, to speed up wire scanners and prepare then for high-intensity operation, and to devise a diagnostic system which can quickly sense a beam hitting components, other than production targets, in the target cells.

A fast-wire scanner has been developed and is ready to be installed and tested in the switchyard. Computer programming modifications are necessary. Progress on toroid integration and centroid monitor development has been reported elsewhere. Heat transfer calculations for harp and wire-scanner wires being heated by intense beans are under way.

\section{Planned Improvements}

As a result of the studies and measurements described above, the following additions and modifications to the switchyard are planned:

1. LAQTS - One triplet will be removed and the other one moved to a more or less central location between the end of the Iinac and $L A-B M-01,-02$. It would be operated in a low-field mode to optimize transmission through Line $A$.
2. $\quad$ AA-BM-01, -02 - These would be moved together as ciose as is practical. Their combined center of bending would, however, remain the same. The purpose of this move is to allow the Line $D$ kicker to be moved farther back while still keeping that beam cut of the LA-BN-02 fringe field. LA-BM02 might be displaced to the side to further benefit Line $D$.

3. Collimator - The collimator and special vacuum plumbing between $L A-B M-01$ and the collimator would be removed and replaced with straight pipes 7.5 or $10 \mathrm{~cm}$ in diameter. Graphite would be stacked around these when and where needed.

4. Line A Direct Beam Stop - A Line A graphite beam stop capable of han'"ing $10 \mu \mathrm{A}$ of beam for several hours and $300 \mu \mathrm{A}$ for short periods of time is needed. It is to be movable so that a Line $A$ direct beam can be sent into Area $A$ after some modest rearrangement of components. The beam stop would normally be outside the switchyard vacuum and have a shielded box for containing it when people are working in that area. To change from the Line $A$ direct mode to delivery of beam to Area $A$ would mean lowering the beam stop, going into the switchyard and closing two valves, inserting a straight-through vacuum pipe where the beam stop normally resides, and reopening the valves to the switchyard vacuun.

5. LX-BM-01 - A hole will be placed at the end of this magnet to permit oppositely charged beams to exit to a new Line $x$ beam stop.

6. New Line $X$ Beam Stop - This stop will accept beams stripped in Line $X$ after LA-BM02. It will be sized for 10- $\mu A$-average current.

7. LX Quadrupoles - Two new quadrupoles should be added to the output of Line $X$ for best matching to Line $C$ requirements at LX-ST-02. These should have apertures larger than $5 \mathrm{~cm}$. The $4016 \mathrm{~s}$ obtained from CEA will work fine. Two of these would be 
placed nearest LX-BM-04. Stands and power supplies will be needed.

8. Strippers - The two Line A strippers would remain in the region between the end of the 1 inac and $L A-B M-01,-02$; two strippers will go in Line $X$ between $L A-B M-02$ and $L X-$ BM-01. Both sets can be used to tailor the phase space for Line $C$. Having two sets gives maximum flexibility. A minimum of three strippers will be required. When it is important to have a Line $A$ beam devoid of any component, $180^{\circ}$ out of phase with the main beam, all the stripping would be done in Line $x$. The present strippers do not allow continuous control of the amount of bean to Line $X$; vertical alignment is also a problem. A new design utilizing the overlap of two $V$-shaped foils is being implemented for LB-ST-01; LA-ST-01 and -02 should be of this new design. A prototype of the new design will be installed and evaluated before the shutdown.

9. Steering Magnets and Beam-Diagnostic Instrumentation - A detailed plan for layout of steering magnets and beam-diagnostic instrumentation in the improved switchyard is being prepared. Improvements in the instruments would be the use of fast-wire scanners, toroid integration for the current monitors, the use of centroid monitors if the developments prove out, and the sizing of wire-scanner wires or foils for high intensity.

10. Switchyard Vacuum Plumbing - Some improvements are planned. Switchyard ion pumps need to be rebuilt. Certain pieces of pipe, bellows, and spools will be increased in size. The problems with thermal cycling of aluminum 0 -ring seals are still being evaluated; replacement by copper seals may be the answer. The window in the switchyard near the TTA must come out. The transition from the relatively poor Area $A$ vacuum of $5 \times 10^{-3}$ torr to the good switchyard vacuum of $10^{-6}$ torr is a problem which needs to be solved. Differential pumping and conductance-limiting baffles are being considered.
lligh-Intensity Modification Program

During the extended shutdown, temporary equipment and shielding will be replaced with updated equipatnt. A list of the major itens to be completed in tne experimental areas is given in Table vil1. As an iid to planning completion and replacement schedules, a PERT nethork is being developed.

Program Evaluation and Review Technique (PERT)

The PERT system is a computer-aided method used for planning, monitoring, and evaluating projects. This program resides on disk at the LASL computing center and is quite easy to use. Four conditions must be satisfied before PERT can be used:

(1) A "game plan" must be developed. The game plan is a sequence of all the activities that must be performed in order to accomplish the project. The shutdown schedule contains, at this stage of development, 800 separate activities that consume tine and require manpower resources. An idditional 350 artivities that consume no time or resources are required to satisfy the logical constraints of the game plan. As the game plan is enlarged to include accelerator and switchyard modifications and secondary bean line radiation hardening, another $200 \mathrm{ac}-$ tivities can be expected.

(2) An individual must be assigned as the person responsible for the successful completion of an activity. This has the considerable benefit of letting everyone know who to turn to for informaticn about that activity. This individual usually has provided the information that is utilized in creating the PERT network, and therefore feels a sense of responsibility for completion of the activity as outlined.

(3) An estimate of the time duration of each activity is required. The PERT program follows along each path of the game plan and adds up the time duration of all the activities. The longest path determines the earliest that the project can be completed. This is known as the critical path. The program then calculates backward from the completion date by subtracting the durations of all the activities along each path. Two dates are then associated with the completion of each activity. The earlier date is the date the activity is expected to be conpleted if the game plan is followed. The second date is the latest one that is allowed for completion of tho activity before the path containing that 
activity becomes the critical path. The time difference between these two dates is known as "slack." Activities lying on the critical path have zero slack. Negative slack is created when the game plan predicts that scheduled comp.etion dates will be missed. The shutdown PERT has slightly negative slack along paths within the EPICS beam line. The goals are to shut the accelerator off on 24 December 1974; turn it back on for accelerator development 1 May 1975; pass beam through Area A on 1 June; and have the capability of suppcrting a high-intensity experimental program by 1 July 1975 . The EPICS spectrometer should be finished 1 November 1975, and the remote-handling tooling should be comoleted 1 March 1976.

(4) An estimate of the manpower required for the completion of each activity may be included. The PERT program uses the date that each activity is expected to be completed, along with the resources required by that activity, and a manpower loading report is generated. This report lists the man-hours required each month for each skill by activity number and gives the slack that exists for that activity. The ability to provide management with information that enables manpower leveling to be accomplished is one of the most useful features of PERT. The shutdown PERT is dominated by the skill requirements outlined in Table VII-II.

Table VII-II, plus lesser manpower requirements of other skills, totals 120500.

PERT therefore provides management with information on schedules, resource requirements, and a means of monitoring progress. It enables management to determine the effects of varying the game plan and to assess the consequences of setbacks such as strikes, technical failures, and fiscal restrictions.

The implementation of a PERT system is a painful process and falls somewhat short of the idealistic picture painted above. Time-duration and resource-requirement estimates must be made for activities that have never been attempted, and are often made by people who have had little experience in estimating.

No matter how bad the game plan or how poor the estimates, PERT still provides an overall picture of the work that needs to be done, approximate1y how Iong it will take, and what is required to do it. As the project progresses and people gain experience, the PERT system will be continually updated to reflect the true state of the project.

TABLE VII-I

MAJOR ITEMS TO BE COMPLETED IN THE EXPERIMENTAL AREAS DURING THE LAMPF SHUTDOWN

-Switchyard Revisions and Line D Installation

-New Target Systems, Vacuum Enclosures, and Collimators for $\mathrm{A}-1$ and $\mathrm{A}-2$

-New Target Box and Collimator for the Biomed Beam

-Main Beam-Stop Replacement

-Radiation-Hard Power and Control Cables

-High-Intensity Beam-Diagnostic Instrumentation

-Remote-Handling Fixtures

-EPICS Beam Line Installation

-Secondary Beam Line Improvements

-Water-Cooled Shielding in Target Cells

-Merrimac Doors

-Full Shielding Cover

-Radiation Damage and Isotope Production Facility

TABLE VII II

SKILL REQUIREMENTS DURING LAMPF SHUTDOWN

\section{$\underline{\text { Ski11 }}$}

Alignment Crew

Draftsmen and Designers

Electricians

MP-1 Electronics Technicians

Pipe Fitters and Plumbers

Ironworkers and Welders

MP-7 Mechanical Engineers

Mechanical Technicians

Crane Operators

Physicists

Machinists
Total Manpower Required (man-hours)

2873

18858

9292

2408

5856

23884

$10 \quad 094$

25500

5830

4040

1360 
VIII. LARGE-SPECTROMETER SYSTEMS

Energetic Pion Channel and Spectrometers (EPICS) Construction

Magnet 1P-BM-02 was scheduled for completion early in this quarter. However, after about six weeks of work, we were unable to make the vacuum chamber leak-tight. At this point, construction was stopped. The pole tips and vacuum chambers for inagnets $1 \mathrm{P}-\mathrm{BM}-02$ and $1 \mathrm{P}-\mathrm{BM}-03$ were sent to the shop for modifications to improve the seal. The new seal is made with $1.5-\mathrm{mm}$ (type 316) stainless steel rather than the 0.5-nim (type 310) previously used. One of the three welds was eliminated to simplify construction. Using the new seal, the vacuum chamber for $1 \mathrm{P}$ BM-03 was completed and the magnet was reassembled. No difficulties have been observed. All remaining magnets will have the new seals.

Bending-Magnet Field Analysis

A version of program MOTER has been prepared which uses a numerical midplane field map as a basis for field computations. The code has been debugged using a map generated with Enge-type field formulas. The new method allows for fields which include terms up to eighth order in $y$ (distance from the midplane). Tests indicate that fourth order is the maximum required. At this order of accuracy, the computing speed using either the map or the field formulas is essentially identical.

We are now engaged in normaliz.7g the existing raw data to the NMR in order to correct long-term drifts in integrator gain and magnet current. The next step will be to use maps from magnet $1 \mathrm{P}-\mathrm{BM}-01$ to compare with alpha-particle data.

Field Mapping

At the beginning of this report period, the decision was made to overhaul the rapid-mapper fieldmeasuring device and to implement some modifications. The project, carried out by personnel in MP-7, included precision alignment of the positioning gear rack and a signal cable take-up system with a hanging weight. The required braided signal cable was made at LASL. These changes brought the mapper back to very good order.

Field-mapping studies were made on channel magnet BM04 during September and October. A new shimming method was tried which involves bolting flexible 1010-steel shims to the field-ciamp noses to produce a desired magnetic "effective field boundary." This "efb" was measured with and without the shims on one side of BM04 to determine if a reasonable amount of shimming material could make the adjustments required to bring this magnet to design performance. More experiments need to be made but the results so far indicate this method could prove effective.

Field maps of BMO4 were made to be used in the ray-tracing code. These maps will be the first to be analyzed at the counting house. This procedure accurately rolibrates mapper data to NMR data in a way that should also allow easier matching of entrance and exit field maps. The coding for this procedure is being debugged.

Measurements have al so been made with the remotely controlled NMR probe. In September the magnet-setting program, written by $C$. Morris of Univ. of Virginia, was tested and parameters were adjusted with the result that the magnets can be set in a reproducible way in 5-10 min. The path of the NMR probe relative to the BMO4 magnet has also been measured. This allows the $\mathrm{H}_{t}-$ winding adjustment program to calculate and try to produce nonuniform field distributions. In the latter part of October $H_{t}$-optimization showed that high field distributions could be reproduced at low fields and the distribution at $17 \mathrm{kG}$ could be made uniform. The techniques evolved in this work will be used to try flattening the 18-kG field. An attempt will again be made to produce sextupole components.

The computer control of the $H_{t}$ regulators and main power supply in the measurement area has been accomplished through the efforts of MP-1. The system is working and is used routinely.

As a part of the continuing effort to upgrade the quality of the field-map data a study has been made on the "pin-find alignment procedure." Data were taken which enabled the coordinates of three pins instead of two to be determined. We decided that the procedure warranted a high degree of confidence but more care would be needed in the drilling of the pin holes. This exercise also led to the discovery of a step-size problem in the mapper. Work to correct this problem is under way. 


\section{Particle Separator}

Power, water, and controls for the separator magnet have been installed in Area A. A new set of maps of the separator magnetic field has been made. Preliminary analysis of these maps indicates that tue magnetic field center is shifted by $\sim 7.5 \mathrm{~mm}$ from the geometric center of the separator box, along the beam direction. A full analysis of these maps and a reanalysis of previous data will be completed after several improvements in the analysis code are made.

The Cockcroft-Walton hv generator has been test run at $400 \mathrm{kV}$ for several hours without problems. An overcurrent protection circuit which provides automatic shutdown of the $\mathrm{CW}$ when its output current exceeds a specified value has been designed and built. When tests on this module are completed, the $C W$ will be moved to Area $A$, installed, and tested again.

The separator hv electrodes have been assembled, installed, and aligned. The hv cable terminations have been designed and are being fabricated. Brazing of two of the three $h v$ insulators is complete.

The separator vacuum box has been tested to $3 \times 10^{-6}$ torr which is adequate for the hv tests. All of the components which will carry $S_{6}$ insulating gas have been pressure tested at $8.5 \mathrm{~atm}$.

\section{Alpha-Particle Test}

During the previous quarter a large amount of $\alpha$-particle data was taken on dipole magnet BMO1. During this quarter, particle trajectories were calculated using field-map data with the program MOTER. There were large discrepancies between the calculation and measurements. Although some differences can be attributed to aligtment mistakes which were found, the discrepancies are still outside of acceptable tolerances. The magnet is now being prepared for alignment rechecks, and, if necessary, for new field measurements.

\section{Magnet-Setting Rate-Dependent Studies}

Studies were made using the B coil in BM04 to determine a setting procedure for the EPICS channel magnets. The eddy-current decay-time constant was found to be on the order of 20 at low fields. Based on these measurements, a magnet-setting program was written which takes care of magnet cycling and overshoots. Further studies showed the rates could be set higher than expected from the $\dot{B}$ coil studies while still maintaining good field reproducibility.

Quadrupole Nagnet Mapping

Water and power have been supplied to the EPICS quadrupole magnet EA-QM-01. Initial measurements of this magnet have been made using the EPICS threedimensional quadrupole magnet mapper. The initial performance of the mapper indicates that fieldmeasurement reproducibility of several parts in $10^{4}$ or better are possible.

\section{Alignment}

During the past several months some preparations have been made for the final alignment of the EPICS channel magnets. These magnets must be installed to within $0.5 \mathrm{~mm}$ of their intended positions in order to avoid any degradation of the design resolution. Alignment fixtures have been attached to the exterior of the magnets. Small pins have been placed in the pole faces to serve as a reference for the magnetic field maps. Direct measurements have been made of all distances between alignment fixtures and mapping pins. All of the above data have been carefully checked for self-consistency and reproducibility. Variances between independent measurements are $\sim 0.08 \mathrm{~mm}$.

With this survey data it was possible to calculate the positions of the mapping pins in the effective field boundary coordinate systems to an accuracy of $0.12 \mathrm{~mm}$ for $\mathrm{BMO} 2$ and $0.1 \mathrm{mn}$ for $\mathrm{BM03}$. It was possible to calculate where the alignment fixtures should be positioned to an accuracy of $0.15 \mathrm{~mm}$ (BMO2) and $0.13 \mathrm{~mm}$ (BMO3). If an accuracy of 127$254 \mu \mathrm{m}$ is assumed for final placement and survey of these magnets, then all errors combined in quadrature should be $0.25-0.40 \mathrm{~mm}$. Similar results should be obtained shortly for BM04. For BM01 some of the above-mentioned surveys were not carried out, and the accuracy of that alignment is in doubt.

\section{EPICS PERT}

The EPICS PERT network was revised several times in order to represent more accurately the work required to complete the channel in time for bean turnon next spring. The delays experience in assembly of $1 \mathrm{P}-\mathrm{BM}-02$ and $1 \mathrm{P}-\mathrm{BM}-03$ and the necessary rework to adapt the other magnets to the new seal technique were also added. The most recent update indicates 
that work on the chainel is about one week behind the target date for beam-on in Area $A$, while work on the spectrometer is three weeks behind the scheduled completion date of 1 October 1975.

\section{EPICS Multipole Magnets}

A design was adopted for three small multipole correction magnets that will be used to trim the channel optics. In particular, these magnets have the ability to correct for the observed gradient which appears in the bending magnet field at excitations above $16 \mathrm{kG}$. In addition, stronger higher order corrections can be made than are presently possible with $\mathrm{H}_{t}$ windings. Two such magnets are under construction at the Univ. of Colorado and the third is being designed.

\section{EPICS Fixed Collimator}

The design of the EPICS front end has been revised to simplify installation and maintenance, improve shielding, and allow space for the new multipole magnets. The early design for the fixed collimator has been scrapped. Poor experience with the Granville-Phillips valve caused us to eliminate this item. The space vacated has been filled with a larger water-cooled collimator which fills all the space between the beam pipe and the target box. This new collimator is built entirely from steel to eliminate the difficulties with a tungsten-to-steel vacuum joint observed earlier. The hole in the collimator has been made $\sim 1.0 \mathrm{~cm}$ larger than the beam in both directions. Calculations indicate that this design minimizes slit-scattered background in the beam and will allow modest misalignment without cutting down the solid angle or increasing background. This new collimator will be fabricated by the Univ, of Virginia users group.

\section{EPICS Beam Lìne Component Rearrangement}

In order to make room for the three new multipole magnets, some changes in the beam line components were required. The most significant change is that the $y$ jaws of the first four-jaw slit ( $1 \mathrm{P}-4 \mathrm{~J}-01$ ) must be replaced with a small vacuum pipe. In addition, four-jaw slit $1 \mathrm{P}-4 \mathrm{~J}-03$ must be moved to the location vacated when $1 \mathrm{P}-4 \mathrm{~J}-05$ was given to the HRS group last year. Calculations indicate that these modifications do not change the slit-scattered background for any cases considered to date.
In addition to the above, we have made provision for a small-y steering magnet to be placed near $1 \mathrm{P}-4 \mathrm{~J}-04$ in order to allow modest misalignment of the bending magnets upstream. A pump-out port will be provided at the exit of $1 \mathrm{P}-\mathrm{BM}-03$ to allow for an additional vacuum pump, if needed. The beam line layout is now frozen and no more changes will be permitted until the construction is complete.

High-Resolution Proton Spectrometer (HRS)

Beam Line $C$

The only remaining magnet to be installed in Line $C, L C-Q M-12$, is in the midst of a series of measurement, chamfer, and shimming operations which should reduce the various integral field-harmonic contributions to $<0.1 \%$ of the primary quadrupole field. Magnet QM12 will be put into place and its water, power, and controls will be installed and checked out. Shimming operations will be done on BM05 during the shutdown period. Other beam line modifications, necessitated by changes in the switchyard, will also be carried out during the shutdown period; the taut-wire system will be added, and the beam line can then be considered in final form.

Although we have not yet demonstrated a "zerophase-space" beam with the strippers in the switchyard, we have obtained distributions which are adequate for beam line tuning purposes. Figure VIII-1 shows an example of an on-1ine data plot (large points) and Fermi-function fit to the data (dots) for beam scanner LC-SZ-03 located about $10 \mathrm{~m}$ downstream from a $50 \mu \times 50 \mu \mathrm{rad}$ beam. The raw data are in the upper plot and the corresponding lialear piecewise derivative is shown in the lower figure. The data are tabulated in the columns to the right, and the peak centroid and first, second, and third moments, etc., are printed in the lower figure in units of $12.5 \mu \mathrm{m}$ so that the peak is located at $3.272 \mathrm{~cm}$ (the beam pipe is $5 \mathrm{~cm}$ in diameter) and the FWHM is $508 \mu \mathrm{m}$.

Magnet measurements on LC-BM-04, the $57^{\circ}$ analyzing magnet upstream of the Line $C$ shield wall, are essentially complete. Shimming in the uniform field regions has given an effective field boundary having the desired shape on the order of $\pm 0.1 \mathrm{~mm}$ peak-topeak across the gap from 5-15 kG. Runs have also been made to study the effect of $H_{t}$ excitation on the effective field boundary. Beam-running cycles 

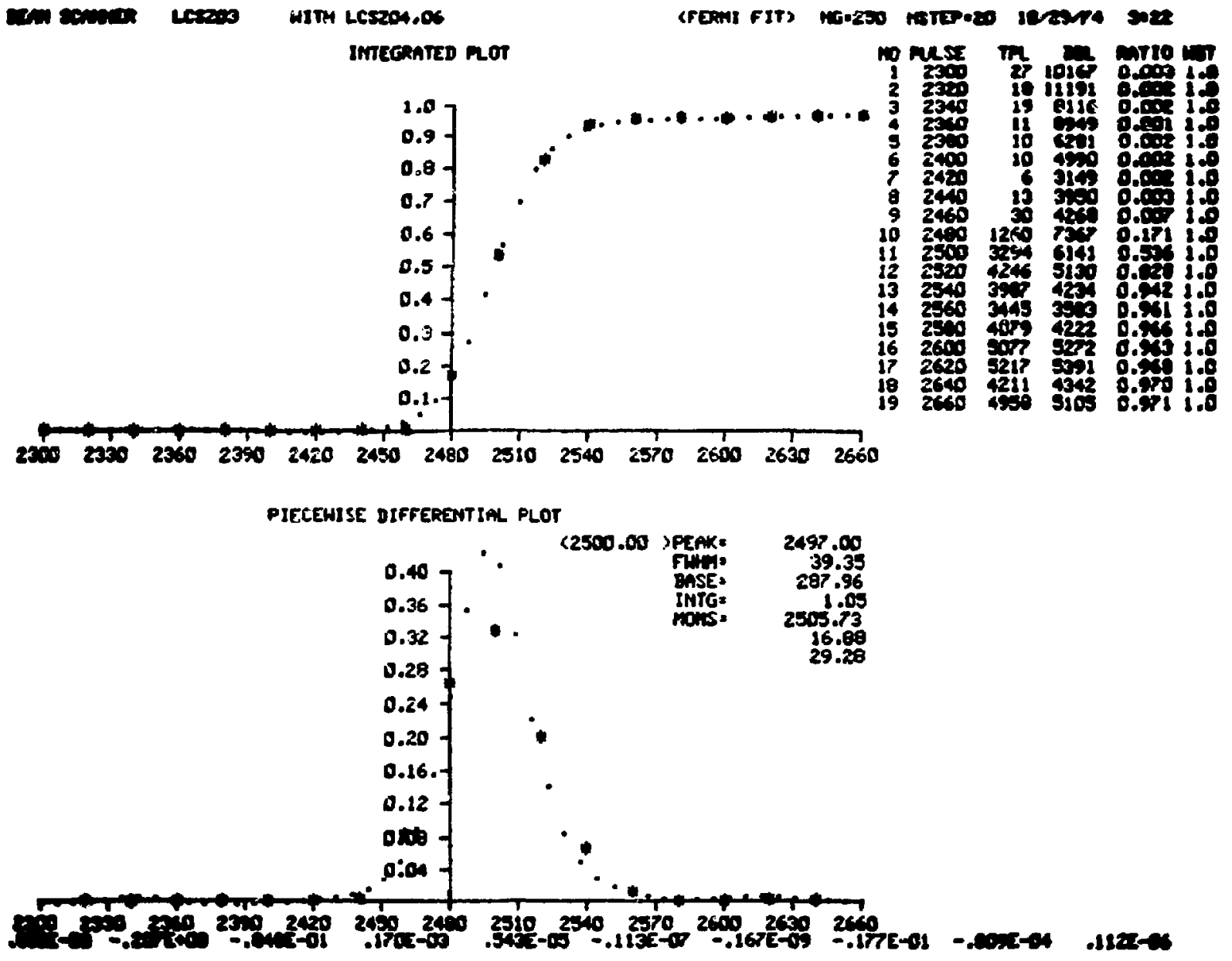
Fig. VIII-1. On-line data plot and Fermi-function
fit for a beam scan with LC-SZ-03.

until the accelerator shutdown will use reduced beam currents and zero phase space to determine the resolution of the line and, if possible, test beam line optimization procedures.

Other progress made during this quarter included redesign, fabrication, and checkout of the NMR drive mechanism and control electronics. All hean 1 ine NMRs will be converted during the next quarter. Spectrometer NMRs will be converted on a noninterference basis. In addition, a considerable amount of design work was accomplished on Line C ISICS, the sliding-seal curtain-retractor mechanism associated with the scattering chamber, and the dc cable layout for the spectrometer. Work was completed on the counting house and orders for all of the racks, the console, and the electronics were placed. Three IBM-729-VI surplus tape drives have been received and installed as has the PDP-11/45 data-acquisition computer.

\section{Spectrometer Dipoles}

The second $75^{\circ}$ spectrometer dipole, IIS-BM-02, which was disassembled and cleaned during the jictvious reporting period subsequent to finding shorts in the upper $\mathrm{H}_{t}$ windings, is now in its final stages of reassembly. Several improvements have been made this quarter in a number of different areas that we hope will finalize the design and thereby permit rigging both dipoies in the spectrometer frame this fiscal year.

The upper ten $\mathrm{H}_{t}$-coils have been fabricated and installed using a different method which virtually eliminates any possibility of $\mathrm{H}_{t}$ shorts to the pole. Aftcr preforming and fitting the conductors to their respective pole slots they were deburred, cleaned, 
prined, and painted with two :... coats of Plasticil whict is water resistant as well as flexible. A check for foreign matier and thin spots was made and they were touched up and allowed to dry. They were then covered with fiberglass sleeving, given another coat of epoxy resin, and allowed to dry. At this point, the slots were carefully deburred, cieaned, and filled with epoxy and the $H_{t}$-coils were inserted so that they were completely immersed. After allowing the epoxy to set, the filler strips were fitted and the slot and strips were again cleaned, with epoxy added prior to final insertion. The resistance to ground of the final configuration was measured to be $10^{9} \Omega$ without water flow in the coils.

In order to reduce the possibility of an external water leak resulting in a short, we have eliminated the center water manifolds. The resulting system has only brazed joints over the body of the magnet except at the entrance and exit manifolds where all connections are made away from the magnet. As a result, any leak can be contained and isolated from the body of the magnet.

In addition, since we were able to trace the gap-height variations in the assembled magnet to correlated variations in the thicknesses of the two pole pieces, a hand-grinding operation on the two pole surfaces was undertaken during this period. Based on mechanical and field contour maps, a grid of 585 points was laid out on each pole face surface ( $\sim 5-x 10-\mathrm{cm}$ mesh). The grinding operation then consisted of removing, to as close a tolerance as possible, the amount of material as specified by the calculations about the grid point in such a way as to hlend into adjacent mesh points. Successive iterations between measurement and grinding resulted in the following distribution of differences $|\Delta Y|$ between the desired and measured gap-removal amounts:

$\begin{array}{rc}\text { Range }(\mathrm{m}) & \text { No. of Points } \\ <|\Delta Y| \leqslant 1 & 281 \\ 1<|\Delta Y| \leqslant 3 & 208 \\ 3<|\Delta Y| \leqslant 5 & 82 \\ 5<|\Delta Y| \leqslant 12 & 11 \\ 12<|\Delta Y| \leqslant 25 & 2 \\ 25<|\Delta Y| \leqslant 38 & 1\end{array}$

If these deviations are integrated through the homogeneous field region at each radius, the result implies a field uniformity of $\pm 10^{-5}$ which is consistent with the design resolution of the spectrometer system. Although it is too early to state whether we can actually reassemble and mount the magnets and obtain this kind of gap-height uniformity in the final system, we believe that this is a valid method applicable to the design of other highresolution systems which, in many instances, will be much less expensive than other possible alternatives. other modifications which were carried out during this period include the welding of the pole-end tips onto the main pole piece and provision for the addition of pole-face shims and antiscattering fixtures to the entrance and exit of each dipole. 
IX. RESEARCH

General Experimental Activity

\section{Secondary Beam Profile Monitor}

The beam profile monitor is now in routine use on Exp. 99. The instantaneous visual presentation provided by the monitor has considerably simplified the set-up and study of the beam. It also provides a continuous monitor of the beam steering and spot shape. A photograph of a profile display obtained recently in the high-energy physics-west $\left(\mathrm{P}^{3}\right.$-west) is shown in Fig. IX-1. Each major division of the oscilloscope graticule corresponds to $13 \mathrm{~mm}$ in space. The beam intensity is $\sim 2 \times 10^{6} \mathrm{particles} / \mathrm{s}$ (average). The chamber is running at $2500 \mathrm{~V}$ with a $70-$ $30 \%$ mixture of argon and carbon dioxide.

Development has started of a similar chamber system which is separated from its electronics by a 3-m umbilical that allows the profile monitor to be placed close to targets or in restricted spots.

\section{Programming for Experiments}

The off-line analysis program for Exp. 96 was modified to include both the upper and lower scattering chambers and scintillation counters. Histograms of target positions, slope, and lab angle were produced using recent data. A program was developed to plot various scalar ratios vs run numbers.

The TRANSPORT code for the 7600 was converted for use on the 6600 under the KRONOS operating system. Several smaller codes were converted from SCOPE to KRONOS.

A computing section was written for the users handbook telling what genera1-purpose computing systems and software are available at LAMPF, how to

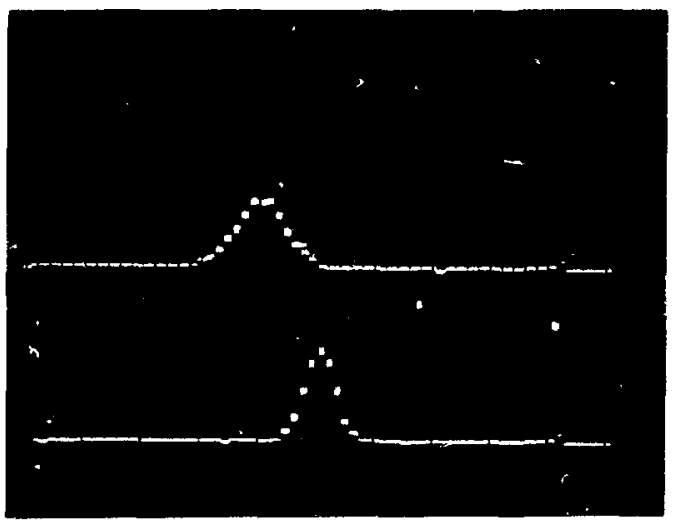

Fig. IX-1. Secondary beam profile monitor. choose a system for a particular job, how to set up simple FORTRAN jobs, and how to submit jobs through the remote batch or keyboard terminals.

\section{Experiments}

\section{Experiment Preparation}

Lepton Conservation Law (Exp. 31)

(Yale Univ., LASL, Saclay, NRC of Canada) Preliminary testing of the $1000-\mathrm{kg}$ water Cereniov counter has been completed, and final assembly is now taking place; 48 of the 96 phototubes have been installed and will be tested shortly. The computer-run light-emitting diode-phototube calibration system is complete and has been tested. Four of the $60-\mathrm{cm} \times 255-\mathrm{cm} \times 2.5-\mathrm{cm}$ scintillation counters for the cosmic-ray anticoincidence system have been installed under the Cerenkov counter and are being tested in place. By November 15 we hope to have all 96 phototubes on the Cerenkov counter and to have four more scintillation counters installed above the Cerenkov counter. Detailed measurements of accelerator-associated neutron backgrounds could then be made during the December run. The water from the X0l magnet system will be filtered a second time before it enters the Cerenkov counter. This will be accomplished by installing a "polishing" unit at the X01 outlet in the biomedical area, and piping the water from there to the neutrino house. The water, once in the counter, will be circulated through a particulate filter.

Gamma Spectra from Pion-and Proton-

Induced Reactions (Exp. 62/121) (Utah State Univ., Univ. Texas, LASL, Florida State Univ., Coll. of William and Mary, ANL)

Extensive preparations for the November-December run are under way at Argonne; perhaps of most importance is a new beam-monitoring method. Locally, the pulse-height analyzers and paper-tape punches have been fixed and tested. Preparations are under way to plot data immediately after each run with the help of the PDP-11 in the low-energy physics (LEP) counting house, a crucial convenience we sorely missed in June.

Minor mechanical modifications to the dewar and shield of one of the GeLi detectors are complete. 
P-P Elastic Scattering (Exp. 132/160) (LASL, VPI, Univ. of Pennsylvania, State Univ. of New York at Geneseo, Texas AGM

Univ.]

We are now in the final stage of assembly for our detectors and the carbon polarimeter in preparation for mounting the system in the external proton beam (EPB) for preliminary runs during November and December 1974.

Our main objectives, in addition to testing all components and the data-acquisition program, are threefold:

1) To test the momentum resolution of our magnetic spectrometer.

2) To verify that a momentum cut by the magnetic spectrometer is equivalent to the coplanarity cut for rejecting quasielastic p-p scattering contributions.

3) To evaluate the performance of our polarimeter under realistic operating conditions, especially with respect to the selection of the range of scattering angles for the $\mathrm{p}-\mathrm{C}$ scattering.

There has been little progress on the polarized target this quarter.

Production Cross Sections of Multiply Charged Fragments from Light Nuclei

(Exp. 179) (LASL, Calif. Inst. of Tech., Univ. of Jew Mexico, Washington State, Munich Tech. Univ.)

Work is proceeding on the heavy ion secondary emission-time pick-off system. The design of the isochronous electron acceleration and transport channel has been improved. A prototype magnet has been constructed. The engineering design of the electrostatic accelerating structure is under way. The channel-plate electron detectors and two of four silicon heavy ion detectors have arrived. The intrinsic time resolution of the TOF electronics has been measured to be 8 ps. The test chamber is complete, except for installation of a vacuum pump.

Tests and Data Runs of Experiments

Pion Total Cross Sections (Exp. 2) Univ. of Montana, Univ. of Washington, New Mexico State, LASL, Univ. of Base1, Stanford Univ.)
The initial run of this experiment took place during April and May. Measurements of total cross sections for both $\pi^{+}$and $\pi^{-}$were made on various nuclei, ranging rrom ${ }^{4}$ He to ${ }^{208} \mathrm{~Pb}$, at energies between 30 and $200 \mathrm{MeV}$. Data were taken simultaneously, both with wire proportional chambers and with an array of transmission counters. The internal consistency of the data has been studied extensively. Programs have been written to correct for accidental counts, counter efficinncies, pion absorption, pion-decay-rate effects, coulomb scattering, and coulomb-nuclear interference, statistical correlations in the data, and other effects. Plans for the next run are also being made for modifications of the experimental apparatus and the techniques used.

P-PSpin-Correlation Experiments (Exp. 27) (Case Western Reserve Univ., Univ. of Southern California, LASL, Univ. of Idaho) Measurements of differential cross sections for elastic scattering of protons by protons have been made at incident beam energies of 647 and 800 MeV. Multiwire proportional chambers (MWPC) detected the scattered and recoil protons in coincidence over the laboratory angular range from 10 to $40^{\circ}$. The data will be reduced to yield cross sections at $\sim 2^{\circ}$ intervals over this range. Polyethylene foils from 3.5 to $18.7 \mathrm{mg} / \mathrm{cm}^{2}$ served as targets and backgrounds were measured by thin carbon foils of equivalent thickness. The beam intensity was monitored by a Faraday cup and an ion chamber. Preliminary analysis indicates that absolute cross sections have been determined to better than $\pm 5 \%$. We expect the final analysis will reduce the uncertainties to $\sim \pm 3 \%$.

Pion-Beta Decay (Exp. 32) (LASL, Temple Univ.)

The calibration of the $\pi^{0}$ detector has been completed. The $\pi^{0}$ detector consists of two $\gamma$-ray detectors, each consisting of a charged-particle veto counter, a lead converter plate, trigger counters, MWPC planes, and lead-glass shower counters. Since this detector requires the gamma rays to interact in the lead converter plates, its efficiency is the product of its geometrical acceptance and the probability of the gamma rays interacting and their showers being detected. The calibration 
was tnade using the reaction $\pi^{-}+p \rightarrow \pi^{0} n$. To allow murc efficient measurement of the detection probability, data were recolded for events having a neutron of the appropriate kinematics to indicate a $\pi^{0}$ of the desires direction and energy and signals from the lead-glass counters in either half of the detector. In addition, to simplify the geometry calculations and iirectly measure the gamma-ray conversion probability, lead frames were installed to limit the lotector area to that of the lead $\gamma$-ray converters. The neutral pion was detected and completely analyzed in enough events to provide an efficiency calibration to the $3 \%$ level.

After completion of the calibration runs, the racuum chamber was installed to look for the reaction signal and background sources in the reactions under study $\pi^{+} \rightarrow \pi^{0}+e^{+}+v_{e}$ or $\pi^{-} \rightarrow \pi^{0}+e^{-}+\bar{v}_{e}$. A variety of $\mathrm{p}^{3}$ output tunes were tried in an effort to minimize beam interaction with the collimator while at the same time optimizing beam flux. Both $\pi^{+}$and $\pi^{-}$beams were studied.

With a reasonably loose trigger, the trigger rate was $\sim 100$ times the expected $\pi-\beta$ decay rate. cuts on $\gamma$-ray energy, coplanarity, timing, and cosmic-ray vetoes eliminate most of the background.

Singles rates in the counters contained a high fraction of real charged particles. Some came from the rear flange and window. The last runs utilized a configuration of the MWPC designed to locate the source of charged particles. These tapes are being analyzed at Temple Univ. The bulk of the charged particles appears to come from either the collimator or the decay region itself, possibly from $\pi_{e 2}$ events. In order to run this experiment at $300 \mu \mathrm{A}$, the charged-particle background from the collimator must be reduced considerably.

Elastic Scattering of $\pi^{+}$from Deuterium
(Exp. 34)
(Univ. Virginia, LASL)

Since the middle of the summer we have been collecting data on $\pi^{+}-d$ elastic scattering at four pion-incident momenta: $350 \mathrm{MeV} / \mathrm{c}, 450 \mathrm{MeV} / \mathrm{c}, 540$ $\mathrm{MeV} / \mathrm{C}$, and $630 \mathrm{MeV} / \mathrm{c}$. The differential cross sec$t i o n$ has been measured at angles ranging from 30 to $120^{\circ}$ in the laboratory, with data taken typically at $10^{\circ}$ intervals in $4^{\circ}$ bins. The statistical accuracy ranges from 5 to $20 \%$, with the lowest accuracy occurring at the larger angles at $630 \mathrm{MeV} / \mathrm{c}$ incident momentum.

The apparatus consists of a magnetic spectrometer to measure the momentum and angle of the scattered pion and a deuteron recoil spectrometer that measures the angle of the recoil deuteron and identifies it with an $E-\triangle E$ system. The targets have consisted of various thicknesses of $C D_{2}$ and $D_{2} D$, so that background from carbon or oxygen is present. This has been measured by taking data with $\mathrm{CH}_{2}$ and $\mathrm{H}_{2} \mathrm{O}$ targets. To serve as an absolute calibration of the apparatus, background measurements were also made on $\pi-p$ scattering. Relative monitoring is provided by an ionization chamber and a nuclear-scattering monitor which are consistent with $1-2 \%$.

Helical-wire proportional chambers are used for measurement of particle trajectories and have performed to our expectations (giving a resolution of the order of $1 \mathrm{~mm}$ ). Consequently, the accuracy of the trajectory measurements is limited by multiple scattering and backgrcund.

Cross sections are not available yet since offline computer analysis of the data is required. A number of corrections must be made, the most significant being corrections for pion decay in the spectrometer, for deuteron break-up in the E- $\Delta E$ system, and for chamber inefficiencies. Data-taking is nearly complete. Data are yet to be taken at a pion angle of $155^{\circ}$ at all four momenta. In addition, we will make some measurements of deuteron break-up cross sections at various energies.

Low-Field Muonium (Exp. 37)

(Yale Univ., LASL, Univ. of Heidelberg, Univ, of Bern, Univ, of Wyoming)

The low-field part of Exp. 37 is a high-precision measurement of the hyperfine structure interval $\Delta v$ in the ground state of the muonium atom $\left(\mu^{+} \mathrm{e}^{-}\right)$. Muonium resonance lines were observed in two lowpressure krypton muns using the separated-oscillating-rields method at 1.8 and $5.1 \mathrm{~atm}$. These data were taken during the run cycles of August 10-19 and August 25 - September 2 and represent about $220 \mathrm{~h}$ of beam time.

Because of the necessity of extrapolating the data to zero gas density, it is desirable to take data at as low a target gas pressure as possible, and the 1.8-atm krypton run represents the lowest 
pressure to date at which muonium resonance curves have been observed.

Extrapolating these two low-pressure points to zero gas density, the following results were obtained: $\Delta v(0)=4463.29835 \pm 0.00253 \mathrm{MHz}(0.57 \mathrm{ppm})$. When these two points are combined with earlier, higher pressure krypton data and fitted to a function of the form

$$
\Delta v(P)=\Delta v(0)\left[1+a p+b P^{2}\right],
$$

the following extrapolated value is obtained:

$$
\Delta v(\mathrm{o})=4463.30090 \pm 0.00204 \mathrm{MHz}(0.46 \mathrm{ppm}) \text {. }
$$

While this represents the most precise measurement of the muoniun hyperfine structure interval in krypton to date, our goal is to achieve $0.2 \mathrm{ppm}$.

In addition to the work with the separated oscillating fields method, two run cycles (September 8-11 and October 25-29) were spent observing resonance curves from "old mucnium," that is, muonium atoms whose decay positrons were gated between 3 and $6 \mu \mathrm{s}$. This technique has the advantage of narrowing the Lorentzian line shape, and, in fact, produces lines two to three times narrower than the standard method of observing muonium.

The target gas used for the first "old muonium" run was 5.1 atm of krypton, and for the second run was $2.3 \mathrm{~atm}$ of krypton. To date, only preliminary analysis has been completed on these runs, and the results indicate this method to be a useful, independent measurement of $\Delta v$ and a check against the systematic errors of the separated oscillating fields method.

Neutron Spectrum and Neutron Scattering (Exp. 56/125)

(Texas AGM Univ., Univ. of New Mexico, LASL, Univ. of Texas)

The major activities this quarter were concerned with two $800 \mathrm{MeV}$ runs which represented continuation of Exps. 56 and 125. In preparation for these runs, improvements were made in the $\mathrm{LH}_{2}$ scattering target and in our on-line and of $f-1$ ine data-handling programs. Data analysis has continued on results obtained earlier; these are noted below. Three papers ${ }^{1-3}$ were presented at the Conference on Few Body Interactions in Quebec in late August of this year.

647-MeV Neutron Spectra at $0^{\circ}$ (Exp. 56). We have obtained data on neutron spectra produced by proton bombardment of deuterium, beryllium, carbon, and aluminum at $0^{\circ}$ neutron angle and incident energies of 647 and $800 \mathrm{MeV}$. Preliminary analysis of the data at $647 \mathrm{MeV}$ has been accomplished. The raw relative spectra for four cases are shown in $\mathrm{Fig}$. IX-2 at $647-\mathrm{MeV}$ incident energy. Corrections have not yet been made for variations in the $n-p$ chargeexchange differertial cross section as a function of

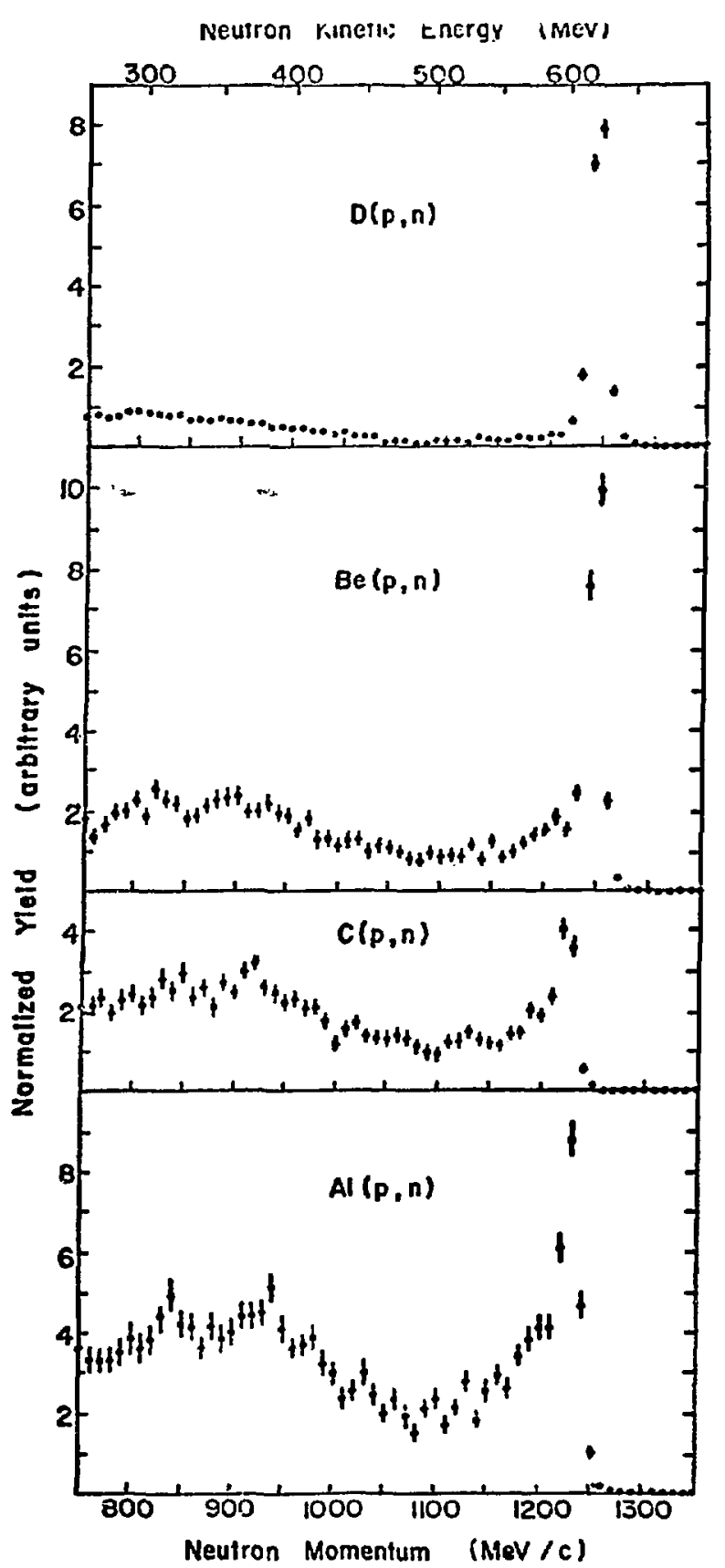

Fig. IX-2, Raw neutron spectra from proton bombardment of $\mathrm{D}_{2}, \mathrm{Be}, \mathrm{C}$, and $\mathrm{Al}$ at $0^{\circ}$ neutron angle and 647-MeV incident energy. 
energy or for nonelastic effects in the $\mathrm{LH}_{2}$ scattering target. The spectra are normalized to the same relative scale. The comparison shows that of these, deuterium has the cleanest high-energy peak and is relatively intense. Beryllium, which may be considered to be two alpha particles and one neutron, shows high peak intensity, but with more low-energy neutrons than deuterium. The peak from carbon is much reduced compared to beryllium, as befits a structure which has properties of three alpha particles. In the aluninum spectrum, a shoulder appears below the peak; we speculate that this may be associated with excitation of the giant quadrupole resonance ${ }^{4}$ in the residual nucleus.

The peak differential cross section for the process $\mathrm{p}+\mathrm{d} \rightarrow \mathrm{n}, 0^{\circ}$ at $647-\mathrm{MeV}$ incident energy, is estimated to be $16 \mathrm{mg} / \mathrm{sr}$. In the vicinity of the $\mathrm{LH}_{2}$ scattering target the radius of the neutron beam is $22 \mathrm{ctm}$ at 0.10 of maximum intensity. The corresponding ne..tron beam intensity is $4 \times 10^{5} / \mathrm{s}-\mu \mathrm{A}$. At $800 \mathrm{MeV}$ the peak production cross section from deuterium appears to be $40 \%$ greater.

Preliminary Results Concerning the Process $\mathrm{p}+\mathrm{p} \rightarrow \mathrm{n}+\mathrm{p}+\pi^{+}$. We have recently analyzed neutron spectra obtained from the reaction $p+p \rightarrow$ $n+p+\pi^{+}$for $0^{\circ}$ neutron angle and incident energy of $766 \mathrm{MeV}$. Neutrons were detected, as in the preceding section, by measuring the momenta of proton recoils induced by $n-p$ charge-exchange scattering. Systematic errors will arise because of the energy

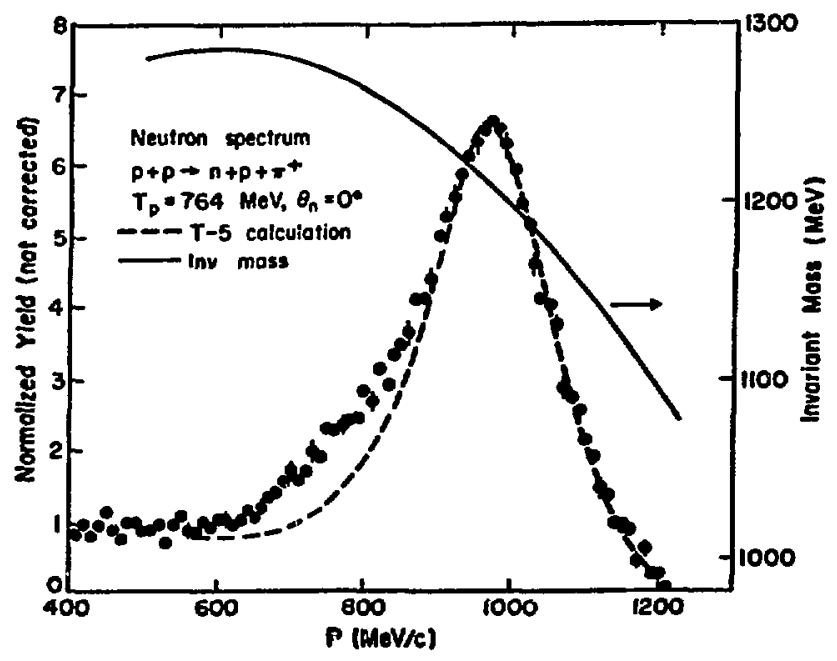

Fig. IX-3. Raw neutron spectrum from the process $p+p+n+p+\pi^{+}$at $0^{\circ}$ neutron angle and $766-\mathrm{MeV}$ incident energy. dependence of the $n-p$ charge-exchange cross section and from recoil protons generated in pion-production processes in the $\mathrm{LH}_{2}$ target. Firm corrections for these effects have yet to be made. Figure IX-3 shows the raw uncorrected neutron-spectrum data on an arbitrary scale. The solid curve in relation to the right-hand scale provides values of invariant mass for the residual $\mathrm{p}-\pi^{+}$pair. At the peak of the spectrum the invariant mass is near $1210 \mathrm{MeV}$, rather close to that of the $\Delta(1236)$. The dashed curve shows calculations of Stephenson, Gibson, and Gibbs of group $T-5$, which reproduce well the qualitative aspects of the spectrum. Other comments on this process appear below in the report on Exp. 124.

Preliminary Results on N-P Charge-Exchange Scattering at 800 and $647 \mathrm{MeV}$. In the past quarter, progress has been made on measurement of the $n-p$ elastic-scattering cross section in the chargeexchange region at 800 and $647 \mathrm{MeV}$. On August 14 and 15 additional data were taken at $800 \mathrm{MeV}$ for $\theta_{1 \mathrm{ab}}$ between $0^{\circ}$ and $56^{\circ}$ in $4^{\circ}$ steps.

Significant progress has been made in the computer analysis of existing $n-p$ cross-section data at $647 \mathrm{MeV}$ for proton recoils in the range $0^{\circ}$ to $4^{\circ}$ (1ab). The detection of deuterons from the reaction $n+p+\pi^{0}+d$ along with the charge-exchange protons provides a convenient basis for an absolute normalization of the $n-p$ cross section, good to $\sim 7 \% .^{5}$ Data were obtained from independent runs at a laboratory scattering angle of $0^{\circ}$ for six different magnet currents. The results are shown in Fig. IX-4. The error bars are statistical and do not include a $7 \%$ normalization uncertainty. A background subtraction has been made for protons and deuterons observed when the liquid hydrogen target was empty.

There is considerable disagreement between previous crass-section measurements in the neighborhood of $650 \mathrm{MeV}$, some of which are shown in the figure. The Dubna values at $630 \mathrm{MeV}^{6}$ are higher than the recent PPA values at $647 \mathrm{MeV},{ }^{7}$ while measurements at Saclay at $\sim 645 \mathrm{MeV}^{8}$ fall somewhere in between. The disagreement is well beyond the normalization uncertainty assigned to each experiment.

our cross sections fall between the PPA values and the Dubna values, and fall on the lower edge of the error band of the LLL phase-shift analysis 9 which was based on the Dubna data. In the c.m. region $172-176^{\circ}$ they appear to be in agreement with 


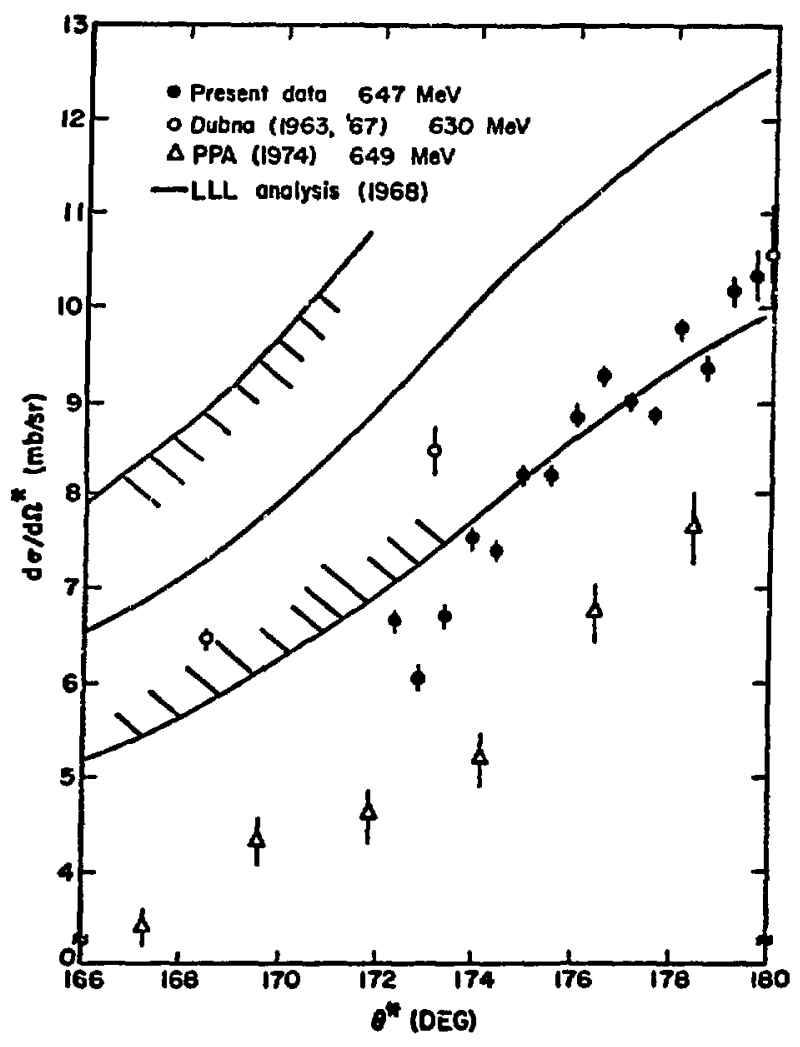

Fig. IX-4. Preliminary data for extreme back-angle $n-p$ scattering near $647 \mathrm{MeV}$.

a parameterization of the Saclay results, but near $180^{\circ}$ our values are somewhat higher.

Chemical Effects in the Capture of Negative Muons (Exp. 60)

(LASL, Princeton Univ.. Munich Tech. Univ., SIN)

Experiments carried out at the stopped muon channel (SMC) since June included the following targets: water (liquid and solid), carbon (hexagonal and tetrahedral), boron nitride (hexagonal and tetrahedra1), para-chloronitrobenzene and paranitrotoluene, alkaline-halide salts, and the oxides $v_{2} \mathrm{O}_{3}, \mathrm{v}_{2} \mathrm{O}_{5}, \mathrm{CaO}, \mathrm{Cr}_{2} \mathrm{O}_{3}$. Much of the data has been analyzed to some degree, and preliminary results are discussed below.

The series water vs ise and the graphitic vs diamond-like forms of carbon and boron nitride dealt with the effects of physical structure. It appears that spectral differences outside of statistical error exist for water vs ice: the $k_{\alpha}$ line is relatively stronger in water, but all other measured $K$ and $L$ lines are relatively stronger in ice.
The carbon data are in the process of being analyzed. The boron nitride data show a relatively stronger $B-K_{\alpha}$ line in the graphitic form and a relatively stronger $\mathrm{N}-\mathrm{K}_{\alpha}$ line in the diamond-like structure. The ratios of captures by boron relative to nitrogen are 0.28 and 0.24 for the graphitic and diamondlike structures, respectively. These capture ratios are to be compared with the (incorrectly applied) Fermi-Teller $2-1$ aw value of 0,71 .

Preliminary results for the alkaline-halide salt targets indicate a decrease in the rate of capture by the cation relative to the anion when going from a solid target to an aqueous solution; however. there is no noticeable conerntration effect over moderate concentrations. The results are what one expects on the basis of the shielding effect provided by the surrounding oxygen atoms from the water molecules. Further details, such as the influence of the type of anion on the relative capture by cation to that by oxygen, are being analyzed.

Upon comparison of the results for $\mathrm{V}_{2} \mathrm{O}_{3}$ and $\mathrm{V}_{2} \mathrm{O}_{5}$, we find the relative capture by vanadium to that by oxygen to be $\approx 20 \%$ higher in the valence- 5 case $\left(\mathrm{V}_{2} \mathrm{O}_{5}\right)$ than in the valence-3 case. The other oxides have yet to be analyzed.

Plans for the remainder of the calendar year include the follo.ing targets: several oxides including $\mathrm{V}_{2} \mathrm{O}_{4}$, gold-silver alloys of three different mixtures, at least two amino acids, and an organic calcium complex.

Development of Pion Beam-Monitoring Techniques (Exp. 67)

(LASL, BNL, ANL, Carnegie-Mellon Univ.)

The data-taking for the first phase of this experiment is nearing completion. This involved the measurement of the absolute cross section of the ${ }^{12} \mathrm{C}\left(\pi^{ \pm}, \pi^{ \pm} \mathrm{n}\right){ }^{11} \mathrm{C}$ monitor reaction over the useful energy range of the $\mathrm{p}^{3}$ channel ( 100 to $550 \mathrm{MeV}$ with the $\pi^{-}$beam and 100 to $470 \mathrm{MeV}$ with the $\pi^{+}$beam) and over the low-energy range of the LEP channel (50- to 220-MeV $\pi^{+}$and $\pi^{-}$). A three-element scintillation counter telescope feeding into fast MECL. 111 discriminators, scalers, and logic circuits was used to count the particle flux passing through thin (3-mmthick) plastic scintillator target disks. The resolving time of the system was 6.5 ns (Fwh of delay curves]. After a 20- to 30-min irradiation the target disk would be rushed to the LAMPF Nuclear 


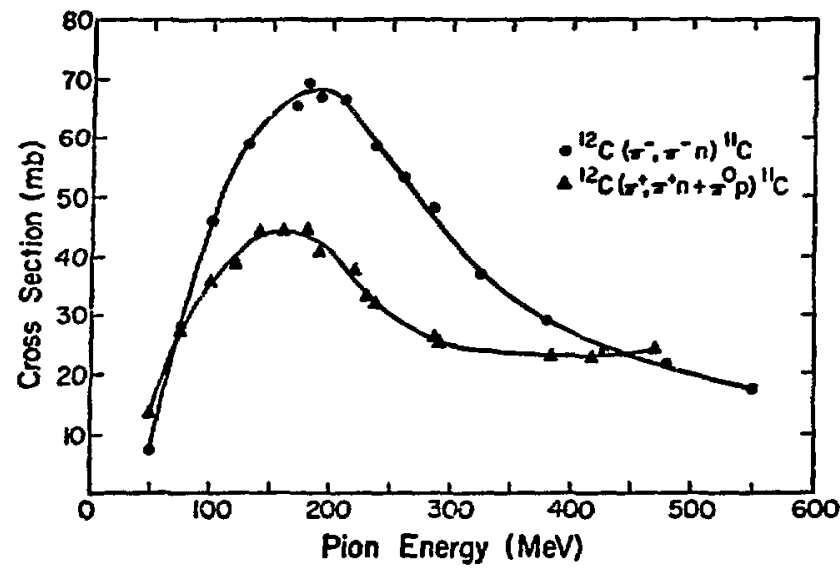

Fig. IX-5, Preliminary cross sections for the $12 \mathrm{C} \stackrel{\pi^{ \pm}}{\rightarrow} 11 \mathrm{C}$ reaction. Each point represents the average of two or more measurements, made on either the $\mathrm{p}^{3}$ or LEP channel.

Chemistry Laboratory where it would be coupled to a phototube, positioned against a NaI(T1) scintillator; and the absolute activity of the induced ${ }^{11} \mathrm{C}$ would be determined by $\beta-\gamma$ coincidence counting. At least duplicate musurements were made at each pion energy; one irradiation generally was made at a high particle rate $\left(22-3 \times 10^{5} / \mathrm{s}\right)$ and one at a low rate $\left(<10^{5} / \mathrm{s}\right)$. Beam contamination (electrons, muons, and protons) was determined either by a TOF measurement employing a midchannel scintillator in conjunction with the triple coincidences from our counter telescope or by a $\mathrm{dE} / \mathrm{dx}$ measurement with a single, thick plastic scintillator at our target location.

A plot of our preliminary data is shown in Fig. IX-5. The ${ }^{12} \mathrm{C} \stackrel{\pi}{\rightarrow}^{11} \mathrm{C}$ excitation function is in good agreement with the earlier work of Reeder and Markowitz, ${ }^{10}$ while the ${ }^{12} \mathrm{C} \mathrm{\pi}^{+}{ }^{11} \mathrm{C}$ excitation function is substantially lower than most previously determined values, The peaks of the 3-3 resonance appear at distinctly different pion energies, 2190 $\mathrm{MeV}$ for $\pi^{-}$and $\sim 160 \mathrm{MeV}$ for $\pi^{+}$. The much-discussed ratio of the cross sections $\sigma \pi^{-} / \sigma \pi^{+}$at the pionnucleon 3-3 resonance appears to have a value of 1.5 , between the of $t$-quoted ratio of 1.0 determined by Chivers et al. 11 and the free-nucleon ratio of 3.0 , but in agreement with the ratio calculated by Sternheim and Silbar. ${ }^{12}$ A crossing over of the two excitation functions appears to occur between 50 and $100 \mathrm{MeV}$. Cross-section values are currently being more accurately corrected for systematic errors and the uncertainties involved are being evaluated.

The second phase of Exp. 67 has commenced and it involves the measurement of the ${ }^{27} \mathrm{AI} \rightarrow^{ \pm} 18 \mathrm{~F}$ cross sections relative to the ${ }^{12} \mathrm{C} \mathbb{I}^{ \pm}{ }^{11} \mathrm{C}$ cross sections. Since ${ }^{18} \mathrm{~F}$ has a relatively long half-life of $1.83 \mathrm{~h}$ compared to $20.4-\mathrm{min}{ }^{11} \mathrm{C}$, and since our preliminary measurements to date indicate that the excitation function for the ${ }^{27} \mathrm{~A} 1 \mathbb{\Pi}^{ \pm} 18_{\mathrm{F}}$ reaction exhibits a broad maximum over the 3-3 resonance, this reaction will serve as a good pion beam monitor.

Elastic-Scattering Amplitude of Pions from Nuclei (Exp. 80)

(Rice Univ., Univ. of Houston, LASL, Columbia Scientific Lab., Washington Univ., M. D. Anderson Hospital

Experiment 80 deternines the real and imaginary parts of the elastic-scattering amplitude of pions from nuclei. At small angles $\left(<10^{\circ}\right)$ the nuclear and coulomb terms of the elastic-scattering amplitude interfere. The magnitude of the interference is proportional to the ratio of the real (Ref $f_{s}$ to imaginary ( $\operatorname{Imf}_{s}$ ) parts of the $\pi^{-}$nucleus-scattering amplitude. Imf $\mathrm{s}$ can be obtained from the total cross section via the optical theorem which allows the real part of the amplitude to be inferred.

Phase one of Exp. 80 has been completed. Data on $\pi^{+}$scattering from ${ }^{40} \mathrm{Ca},{ }^{208} \mathrm{~Pb}$, and ${ }^{12} \mathrm{C}$ at energies from $100-220 \mathrm{MeV}$ on the $\mathrm{P}^{3}$ beam have been taken and are in final analysis. Figure $I X-6$ shows the experimental setup. The incoming particle angle and position are measured by the two multistrip scintillation counters (MSSC). The magnetic spectrometer consisting of six 2-D MWPC, Ames-C magnet, and $30 D 40$ magnet was used to measure momentum and angle of the scattered particles. The momentum resolution of the spectrometer was $1.3 \%$ FWHM; the $\mathrm{p}^{3}$ bearn has a momentum spread $\Delta \mathrm{p} / \mathrm{p}=2 \% \mathrm{FWHM}$. The scattering angle is determined with a resolution of $0.25^{\circ} \mathrm{FWHM}$. The spectrometer's effective solid angle allowed the measurement of angular distributions with a width of $5^{\circ}$. Thus a complete angular distribution from $5^{\circ}-24^{\circ}$ typically required four spectrometer settings $\left(7,12,17\right.$ and $\left.22^{\circ}\right)$. 


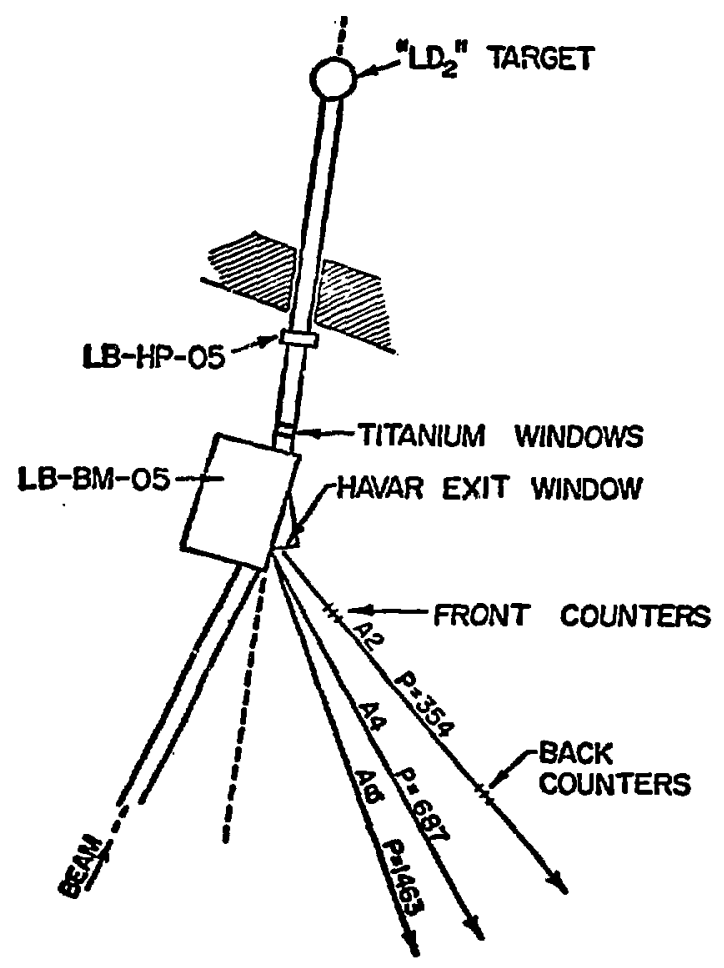

Fig. IX-6. Setup for Exp. 80. $\left(P_{1}-P_{6}\right.$ are twodimensional MWPCs; $T$ is the scattering target; $S_{1}$ and $S_{2}$ are fast plastic scintillators (NE-102) which are used as a fast trigger for the MWPC readout logic. The MSSC consist of fifteen $0.3-\mathrm{cm}-w i d e$ scintillation strips in $x$ and $y$; they are used to define the incoming pion trajectory.)

Figure IX-7 is an angular distribution of $\pi^{+}$${ }^{40} \mathrm{Ca}$ scattering at a pion kinetic energy of $190 \mathrm{MeV}$. The differential cross section do/d $\Omega$ is in arbitrary units because final calculations for the solid angle and purity have not been completed. The Imf $f_{s}$ was determined by measuring the total cross sections for $\pi^{+}-{ }^{40} \mathrm{Ca}$ and $\pi^{+}-\mathrm{C}$ over the same energy range. Analysis of the small-angle data is continuing, with completion and publication expected in the near future.
$\mathrm{PI}^{+} \mathrm{CA} 40$ $190 \mathrm{MEV}$

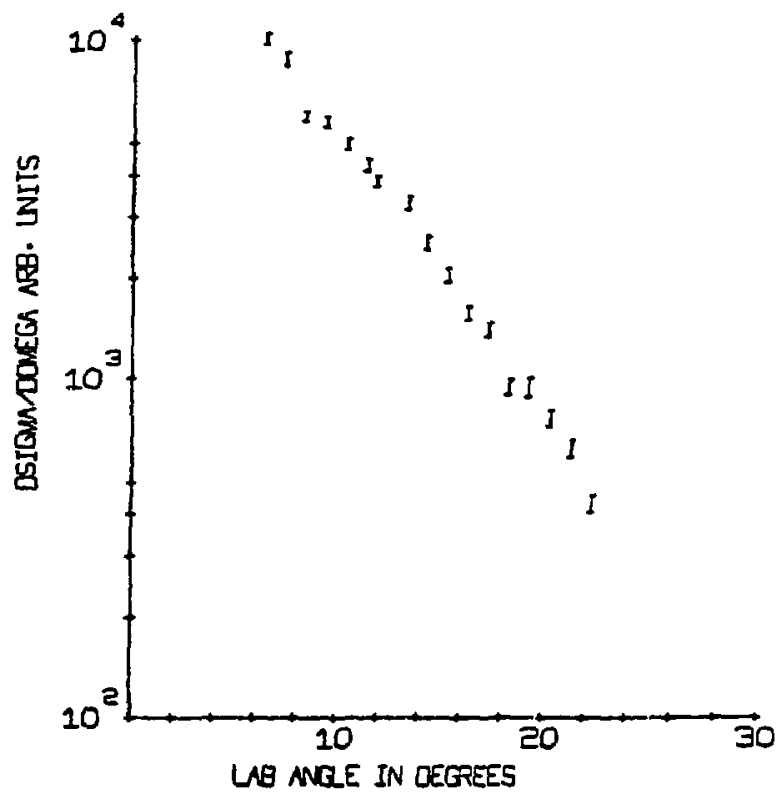

Fig, IX -7 . Preliminary results of $\pi^{+}-40$ Ca elastic scattering at $190 \mathrm{MeV}$.

Study of Neutron-Proton and Proton-Proton Coincidence Spectra from the P + D $\rightarrow$ $\mathrm{N}+\mathrm{P}+\mathrm{P}$ Reaction (Exp. 81) (Rice Univ., Univ, of Houston, LASL, Columbia Scientific Lab., Washington Univ., M. D. Anderson Hospita1, NSF)

Measurement of $n-p$ and $p-p$ coincidence spectra from proton-induced deuteron break-up will yield valuable information on the mechanisms for nuclear reactions with three particles in the final state. Both the final state interaction (FSI) and the quasifree scattering (QFS) processes were studied. The same experimental setup was also used to study the pion-production reaction $p+p \rightarrow n+n+\pi$.

The experimental arrangement consisted of a liquid hydrogen target placed in the EPB line. Data were taken with a magnetic spectrometer arm in coincidence with a TOF arm. The proton trajectories were determined by MWPC. A neutron counter was placed behind the rof arm. The neutron counter was in coincidence with the spectrometer arm. The $p-n$ and p-p coincidence spectra were measured simultaneous ly. 
TABLE IX-I

ANGLE PAIRS FOR $\mathrm{P}+\mathrm{D} \rightarrow \mathrm{N}+\mathrm{P}+\mathrm{P}$ DATA RUNS QRS FSI

\begin{tabular}{ll}
${ }_{\mathrm{p}}^{\mathrm{l}}$ & $\theta_{\mathrm{p}, \mathrm{n}}^{2}$ \\
\hline 30 & 30 \\
34 & 34 \\
40 & 40 \\
44 & 44 \\
48 & 48 \\
52 & 52 \\
45 & 32 \\
50 & 30 \\
55 & 25
\end{tabular}

Because of the relatively low duty factor of the LAMPF proton beam, the August runs were intended to be an exploratory experiment to identify problems and assess the feasibility of the technique. Considerably more was accomplished. The low duty factor did result in such severe accidental problems that the proton beam had to be reduced to $5 \mathrm{pA}$. Furthermore, pile-up rejection circuitry was needed to eliminate double tracks within the 200-ns livetime of the MWPCs. Within these limitations, adequate count rates could be achieved and data were accumulated for the angle pairs shown in Table IX-I. Thus the QFS and FSI experiments at $800 \mathrm{MeV}$ have been completed and analyses of the data are under way. Preliminary results show a strong FSI peak.

A short series of $p+p+n+n+\pi$ runs demonstrated the feasibility of this experiment with the present equipment.

\section{Counter Experiments in the Thin Target}

$$
\text { Area (Exp. 86) }
$$$$
\text { (Texas AEM Univ., ORNL, BNL, LBL, LASL) }
$$

Double differential cross sections $\frac{\mathrm{d}^{2} \sigma}{\mathrm{dEd} \Omega}$ have been measured for helium, lithium, beryllium, and boron nuclides produced in the interaction of 800 MeV protons with a $3.5-\mathrm{mg} / \mathrm{cm}^{2}$ self-supporting uranium target (made at $L B L$ ) in the Line $A$ Thin Target Area (TTA), which is located in the proton switchyard. Individual nuclides were identified by means of energy loss and total energy measurements in silicon detector telescopes located in evacuated detector boxes $4.6 \mathrm{~m}$ from the target. The multiparameter data were stored event by event on magnetic tape, and subsequent off-line processing of the data resulted in adequate particle identification.

Preliminary results indicate that the energy spectra of helium-boron fragments peak at somewhat higher kinetic energies and fall off more steeply above the peak than earlier results from comparable experiments at $5 \mathrm{GeV}$ at Berkeley. Angular distributions and production cross sections will be obtained from the data.

Additional equipment will be jnstalled in November to provide the capability of measuring the flight times of nuclear fragments over a $25-\mathrm{cm}$ flight path. This will allow energy distributions to be measured to lower energies and thus unique particle identification for nuclear fragments heavier than boron will be possible.

Low-Energy ip Elastic Scattering (Exp. 96) (LASL, Arizona State Univ.)

The summer data run of Exp. 96 has been completed. The LEP channel was used from the end of July to mid-October (except for one cycle in August) to accumuiate elastic $\pi p$ scattering data at 30,40 , 50 , and $70 \mathrm{MeV}$. Over 10000 events of interest were accumulated at each energy and polarity. Figure IX-8 shows a schematic of the apparatus which was used. The scattering system consisted of an up-down symmetrical arrangement of detectors which viewed events which came from the region of the $\mathrm{H}_{2}$ target. Both the up and down scattering systems contained three MWPCs with wire spacing of $2.0 \mathrm{~mm}$ and nine scintillation counters (SCINT) arranged into three groups each with two energy-Ioss counters and a total energy counter. An incoming beam particle

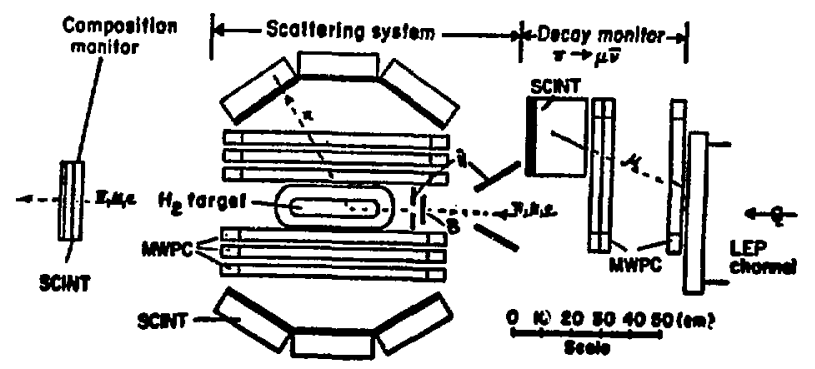

Fig. IX-8. Tp elastic-scattering experimental setup. 
was signalled by a 0,4-mm-thick scintillation coun$\operatorname{ter}(B)$. Halo counters $(H)$ eliminated those particles which were outside of the main beam.

The decay monitor consisted of three MWPCs and a total-energy scintillation counter. A counter directly behind the energy counter eliminated those particles which were nut stopped by the total energy counter. This system measured the number of $\pi \rightarrow \mu \bar{v}$ decays which came from a vacuum tank drift region at the end of the LEP channe1. The composition monitcr consisted of three energy-loss scintillation counters. It measured the number of pions, muons, and electrons in the beam.

livents of interest were recorded on magnetic tape when a particle satisfied a $2 / 3$ chamber plane requirement in each of two orthogonal MWPC planes, a $3 / 3$ (sometimes $2 / 3$ ) scintillation counter requirement (two energy loss counters and a total energy counter), and a beam counter but no beam halo requirement. Chamber information, counter pulse heights, and register information were recorded on every taped event. All chamber, counter, channel setting, and target parameter voltages were recorded on tape every $10 \mathrm{~min}$; all scalers were put on tape every $10 \mathrm{~s}$; and beam composition and pion-intensity monitoring was interspersed with normal logic events at a rate of approximately one monitor count to every 10 scatter-type events.

Al1 equipment, with the exception of the $\mathrm{H}_{2}$ target, gave excellent reliability. The $\mathrm{H}_{2}$ target was run typically at $\imath 1.5$ atm of pressure and at temperatures between $20^{\circ} \mathrm{K}$ and $30^{\circ} \mathrm{K}$. The improved $\mathrm{H}_{2}$ target flask, of approximately elliptical cross section of $8 \mathrm{~cm} \times 14 \mathrm{~cm}$, greatly reduced the number of background events from the side walls compared to what was seen by a smaller flask (see LA-5580-PR, Quarterly Report on the Medium-Energy Physics Program for the Period Ending January 31, 1974). The MWPCs amplificr and encoder systems, once debugged, worked quite well. The scintillation counters and LEP channel proved to be almost trouble free.

Currently, the analysis program is being developed. The data look good. The agreement between the two systems for beam monitoring is good, and all aspects of the pion flux decay monitor are being scrutinized for eventual sole use at higher beam intensities.

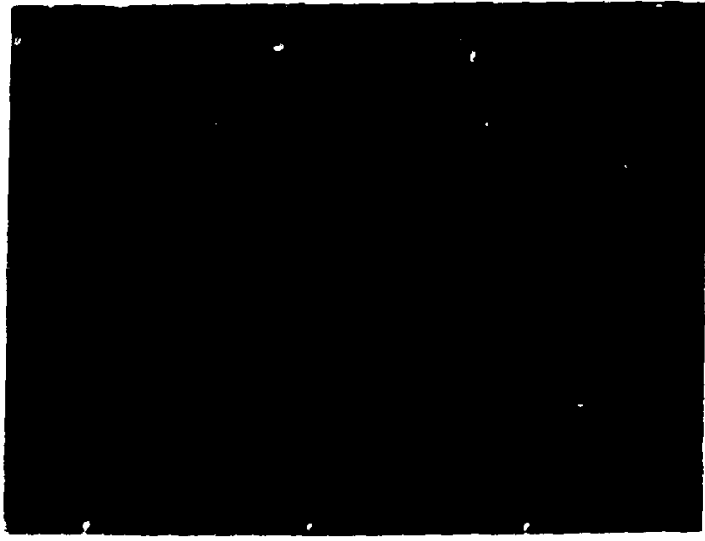

Fig. IX-9. Profile of $\pi^{+}$pions (of 240-MeV momentum) scattered from a polystyrene target.

The $\pi^{-}+\mathrm{p}+\pi^{-}+\pi^{+}+\mathrm{n}$ (Exp. 99) Colorado Col1., Univ. Virginia, LASL, Univ. Wyoming

At the beginning of this quarter tests of the spectrometer using an $241_{A m} \alpha$ source were performed. With a 1-mm-high source a resolution of $0.17 \%$ was obtained. The effects of detector placement and slit setting were observed as well, to be compared with similar data taken with a real pion beam and target.

Experiment 99 is now installed and running. Shakedown and calibration of the equipment using a solid target is nearly complete. We plan to install the liquid $\mathrm{H}_{2}$ target and begin data-taking early in November. We are currently waiting on the construction of an iron shield house on the magnet.

The spectrometer and detector system have performed as expected. A profile of positive pions (of $240-\mathrm{MeV} / \mathrm{C}$ momentum) scattered from a polystyrene target is shown in Fig. IX-9. The two peaks are due to pions elastically scattered from carbon and from hydrogen at $37^{\circ}$. In this case the resolution is dominated by the momentum acceptance of the pion channel, and contributed to by the vertical spot size. (The ninth detector is not operating in this picture.) A series of profiles of this genre has been recorded for various settings of the entrance slits and with several adjustments of the $\mathrm{p}^{3}$ channel. Calibrations of beam monitors and timeof-flight studies of beam compositions are heing done concurrently with the spectrometer tests. 
Spallation-Yield Distributions from Pion

Interactions with Complex Nuclei (Exp. 103) (BNL, LASL)

This experiment is a study of pion-nucleus reactions through measurements of the yields of radioactive products resulting from $\pi^{+}$and $\pi^{-}$bombardments of copper, niobium, and higher $Z$ target elements. Despite the low pion beam intensities available, a few preliminary irradiations of copper targets ( $1.5 \mathrm{~g} / \mathrm{cm}^{2}$ ) with $190-\mathrm{MeV} \pi^{+}$and $\pi^{-}$beams from the $\mathrm{p}^{3}$ channel have been obtained. The formation crosi sections for numerous high-yield spallation products were measured by $\mathrm{Ge}(\mathrm{hi}) \gamma$-spectrometry techniques. Crc s-section values are put on an absolute basis by comparison with the pion beam monitors, ${ }^{12} \mathrm{C} \stackrel{\pi}{\rightarrow}^{ \pm} 11_{\mathrm{C}}$ or ${ }^{27} \mathrm{Al} \stackrel{\pi^{ \pm}}{\rightarrow} 18 \mathrm{~F}$, as determined absolutely in Exp. 67.

Two proton bombardments of copper and niobium have been performed for direct comparison of protoninduced spallation product yields with those induced by pions. The incident proton energy was chosen to be $349 \mathrm{MeV}$, approximately the sum of the pion kinetic and rest mass energies. Preliminary results indicate that the yields of all $19 \gamma$-emitting products from the $190-\mathrm{MeV} \pi^{+}$bombardment are higher than those from the $349-\mathrm{MeV}$ proton irradiation.

Yields of the residual products from the reactions $\mathrm{Cu}+\pi^{+}(200 \mathrm{MeV})$ and $\mathrm{Cu}+\mathrm{p}(340 \mathrm{MeV})$ have also been calculated through the use of the VEGAS intranuclear cascade plus evaporation codes. Calculated yields are in fair agreement with the preliminary $\pi^{+}$experimental results and $w i$ th the proton results. A much more comprehensive study of these complex pion-nucleus reactions will be carried out when substantially higher pion beam intensities become avai lable.

\section{Nuclear Spectroscopy Studies of Proton- Induced Spallation Products (Exp. 105) (Idaho National Engineering Lab., LASL)}

A number of short-lived neutron-deficient nuclides, some of which are rather far from $B-$ stability, have been produced for off-line study, by means of spallation reactions induced by high-eriergy (500-800 MeV) proton bombardment of heavy element targets. Some of the bombardments have been carried out using a target facility ahead of the line $A$ beam stop in the switchyard; others have been made at a target station installed in front of the Line $B$ beam stop. Average beam currents as high as $30 \mu \mathrm{A}$ have been utilized in the Line A bombardments, but the maximum intensity ayailable thus far at the Line $B$ station is $\sim 1 \mu \mathrm{A}$. Integrated target exposures have ranged up to $20 \mu \mathrm{A}-\mathrm{h}$. The targets most commonly used have been tantalum or praseodymium metal foils, $200-300 \mathrm{mg} / \mathrm{cm}^{2}$.

The bombarded targets are either transported to LASL Group CNC-11 or are shipped by air to a team at Idaho Falls. Specific nuclides are then isolated for study through a combination of chemical purification and isotope separation procedures. "sable quantities of over 100 isotopes are typically produced in a single bombardment, the production cross sections for individual species being in the range of $1-100 \mathrm{mb}$.

Most of the species selected thus far for spectroscopic studies are barium, lanthanum, and cerium nuclei in the mass region $A=128-137$, and gadolinium, terbium, dysprosium, and erbium nuclei with $A=146-158$. In these regions, the nuclear equilibrium shape is changing from spherical to deformed, and the nuclear coupling scheme is not yet we1l-defined. Detailed analysis of the numerous high-resolution $\beta$ - and $\gamma$-ray spectra that we have recorded should lead to an improved understanding of these transitional nuclei. In our work to date, many new energy levels have been found. For example, in ${ }^{151}$ Gd five new energy levels have been established and seven of the previously proposed levels have been shown not to exist.

Emphasis has been placed on the determination of total decay energy $\left(Q_{R}\right)$ values, which yield nuclidic masses. Work on ${ }^{158} \operatorname{Er}(2.4$ days $) \rightarrow{ }^{158_{\text {Ho }}}$ and ${ }^{128} \mathrm{Ba}(2.4$ days $) \rightarrow{ }^{128} \mathrm{Cs}(4 \mathrm{~min}) \rightarrow{ }^{128} \mathrm{Xe}$ is essentially complete. In the case of ${ }^{158} \mathrm{Er}$ decay, we have established that $Q_{B}$ is $>300 \mathrm{keV}$ less than the literature value. Also, no evidence can be found for $B^{+}$emission, in conflict with previous reports from the USSR. For ${ }^{128} \mathrm{Ba}$, we measured for the first time values of $Q_{B} \cdot{ }^{13}$ The mean value is 2370 keV less than the semiempirical estimate of Wapstra and Gove.

Other results include the observation, for the first time, of weak $\beta^{+}$emission from ${ }^{137} \mathrm{Ce}(9 \mathrm{~h})$, ${ }^{153} \mathrm{~Tb}\left(2.4\right.$ days), and ${ }^{1568} \mathrm{~Tb}(5.1$ days), all of which 
decay predominantly by electron capture. An inportant consequence of these observations is that experiments can now be devised to determine the $Q_{B}$ values.

Search for New Neutron-Rich Nuclides

Produced by Fast Neutrons (Exp. 111) (Texas AEM, Baylor Univ., Univ. Ok lahoma)

It is difficult to produce neutron-rich nuclei with $Z<32$ or $Z>64$ by conventional means (charged particles), but it might be possible to produce such nuclei (outside of the fission region) using large fluxes of high-energy neutrons generated at the LAMPF beam stop $A$. The reactions $(n, 2 p n),(n, 2 p)$ and $(n, 3 p n)$ would be very useful in producing new neutron-rich species.

The yields of the above reactions were measured in the mass region below $A=142$. Targets of suitable materials were encapsulated in polyethylene and irradiated $i_{i}$ the "rabbit 1ine" adjacent to the beam stop at $90^{\circ}$ to the beam's direction and $203 \mathrm{~mm}$ from the center of the beam 1 ine. In each run, ${ }^{28} \mathrm{Mg}$ $(21.1 \mathrm{~h})$ from the ${ }^{31} \mathrm{P}(\mathrm{n}, 3 \mathrm{pn})^{28} \mathrm{Mg}$ reaction was used as a vield standard. The yield of the above reaction was measured relative.to that of ${ }^{11} \mathrm{C}$ in ${ }^{12} \mathrm{C}(\mathrm{n}, 2 \mathrm{n})^{11} \mathrm{C}$. Typical beam currents were $4 \mathrm{HA}$ of $800-\mathrm{MeV}$ protons at the beam stop. Gamma rays from isotopes of interest were measured using a $\mathrm{Ge}(\mathrm{Li})$ detector. In most cases postirradiation radiochemical separations were necessary because of the high yield of neut ror-defjcient species. Chemical separation yields were measured by neutron activation of the carrier and a standard at the beam stop.

The following reactions were observed:

$$
\begin{aligned}
& { }^{31} \mathrm{P}(\mathrm{n}, 2 \mathrm{pn}){ }^{29} \mathrm{~A} 1 \\
& { }^{45} \mathrm{Sc}(\mathrm{n}, 2 \mathrm{pn}){ }^{43} \mathrm{~K} \\
& { }^{75} \mathrm{As}(\mathrm{n}, 2 \mathrm{pn}){ }^{73} \mathrm{Ga} \\
& { }^{133} \mathrm{Cs}(\mathrm{n}, 2 \mathrm{pn}){ }^{131} \mathrm{I} \\
& { }^{142} \mathrm{Ce}(\mathrm{n}, 2 \mathrm{pn}){ }^{140} \mathrm{Ba} \\
& { }^{45} \mathrm{Sc}(\mathrm{n}, 2 \mathrm{p}){ }^{44} \mathrm{~K} \\
& { }^{89} \mathrm{Y}(\mathrm{n}, 2 \mathrm{p}){ }^{88} \mathrm{Rb} \\
& { }^{133} \mathrm{Cs}(\mathrm{n}, 2 \mathrm{p}){ }^{132} \mathrm{I} \\
& { }^{139} \text { La }(n, 2 p){ }^{138} \mathrm{Cs} \\
& 31_{\mathrm{P}(\mathrm{n}, 3 \mathrm{pn})}{ }^{28_{\mathrm{Mg}}} \\
& { }^{41} \mathrm{~K}(\mathrm{n}, 3 \mathrm{pn})^{38} \mathrm{~S} \\
& { }^{75} \mathrm{As}(\mathrm{n}, 3 \mathrm{pn})^{72} \mathrm{Zn} \\
& { }^{115} \operatorname{In}(\mathrm{n}, 3 \mathrm{pn}){ }^{112} \mathrm{Pd}
\end{aligned}
$$

If one assumes a cross section of $10 \mathrm{mb}$ for the ${ }^{12} \mathrm{C}(\mathrm{n}, 2 \mathrm{n}){ }^{11} \mathrm{C}$ reaction, the following trends are evident. The cross section for ${ }^{31} P(n, 2 p n)^{29} A 1$ is 5.8 mb. As A decreases, the cross section for $(n, 2 p n)$ falls slowly, about a factor of 30 over the range studied. Cross sections for the $(n, 2 p)$ reactions also decrease slowly with increasing $A$ and are typically a factor of 10 smaller than the corresponding $(\mathrm{n}, 2 \mathrm{pn})$ cross sections. The cross section for ${ }^{31} \mathrm{P}(\mathrm{n}, 3 \mathrm{pn})^{28} \mathrm{Mg}$ is $0.3 \mathrm{mg}$ but $(n, 3 \mathrm{pn})$ decreases rapidly with increasing $A$. As an example ve could not detect ${ }^{135} \mathrm{I}(6.7 \mathrm{~h})$ from the ${ }^{138} \mathrm{Ba}(\mathrm{n}, 3 \mathrm{pn})^{135} \mathrm{I}$ reaction.

A study of the decay chain $200 \mathrm{Pt}(14 \mathrm{~h})+200 \mathrm{Au}$ $(48 \mathrm{~min}) \rightarrow{ }^{200} \mathrm{Hg}$ is being made using Ge(Li) detectors. The ${ }^{200} \mathrm{Pt}$ was produced by the ${ }^{204} \mathrm{Hg}(\mathrm{n} \text {, an })^{200} \mathrm{Pt}$ reaction using a separated ${ }^{204} \mathrm{Hg}$ target. The platinum was chemically separated from mercury, gold, and fission-product impurities. A new half-life of $14 \mathrm{~h}$ for ${ }^{200} \mathrm{Pt}$ is longer than the old value of $12 \mathrm{~h}$. Gamma decay of ${ }^{200} \mathrm{p}_{t}$ has been observed for the first time but the $B$ feeding to excited states in ${ }^{200} \mathrm{Au}$ is weak $(<10 \%)$

A search for the undiscovered nuclide ${ }^{202} \mathrm{Pt}$ is under way using the $\left.{ }^{204} \mathrm{t}_{0}, \mathrm{n}, 2 \mathrm{pn}\right)^{202} \mathrm{pt}$ reaction. Since the half-1ife of ${ }^{202} \mathrm{Pt}$ is expected to be $<20$ min, a fast radiochemical procedure was developed that separates platinum from mercury, gold, and fission products in about $5 \mathrm{~min}$. Searches for ${ }^{202} \mathrm{pt}$ using separated ${ }^{204} \mathrm{Hg}$ targets have been inconclusive due primarily to the present low proton beam currents $(\sim 5 \mu \mathrm{A})$ at the beam stop. Searches for this and other neutron-rich nuclides will continue when higher neutron fluxes become available.

Fragment Emission from Pion Interactions with Complex Nuclei (Exp. 118) (Purdue Univ., LASL]

This experiment is a study of the so-called "fragmentation" process, whereby a large chunk of nuclear matter is ejected from a heavy nucleus by the interaction of a very energe:ic incident projectile. Specifically, the yield of $15-\mathrm{h}{ }^{24} \mathrm{Na}$ from the interaction of $100-$ to $400-\mathrm{MeV}$ pions . (+ and -) with targets of copper, silver, gold, and uranium wil! be measured and compared with the yields from proton interactions. Since substantially higher pion beam intensities than presently available are 
needed to do the pion study, the proton counterpart was started. Targets of high-purity gold were bombarded with 800-, 597-, 398-, and 197-MeV protons in Line $B$ and Line $A$ Direct and the ${ }^{24} \mathrm{Na}$ was radiochemically separated and measured by Ge(Li) $\gamma$-counting. The cross sections for the ${ }^{24} \mathrm{Na}$ in aluminum beam monitor foils are shown in Fig. IX-10. The yields of ${ }^{24} \mathrm{Na}$ from pion-induced reactions are expected to provide some insight into the mechanism of this complex nuclear reaction.

Nuclear Structure Effects in Pion-Induced Nuclear Reactions (Exp. 123). (CarnegieMellon Univ., Reed Co11., LASL]

Since this study is based on a comparison of pion and proton reactions on a collection of target systems whose detailed nuclear properties vary in a known way, it was important that the proton experiments commence while waiting for adequate pion beam intensities. A series of 11 proton-activation experiments were carried out at $800 \mathrm{MeV}$ in the Area $B$ Nuclear Chemistry Cave on the enriched medium mass target isotopes: ${ }^{90} \mathrm{Zr},{ }^{96} \mathrm{Zr},{ }^{96} \mathrm{Mo},{ }^{96} \mathrm{Ru}$, and ${ }^{102} \mathrm{Pd}$. Yields of the spallation nuclides are being determined from analysis of Ge(Li) $\gamma$-spectrometry data.

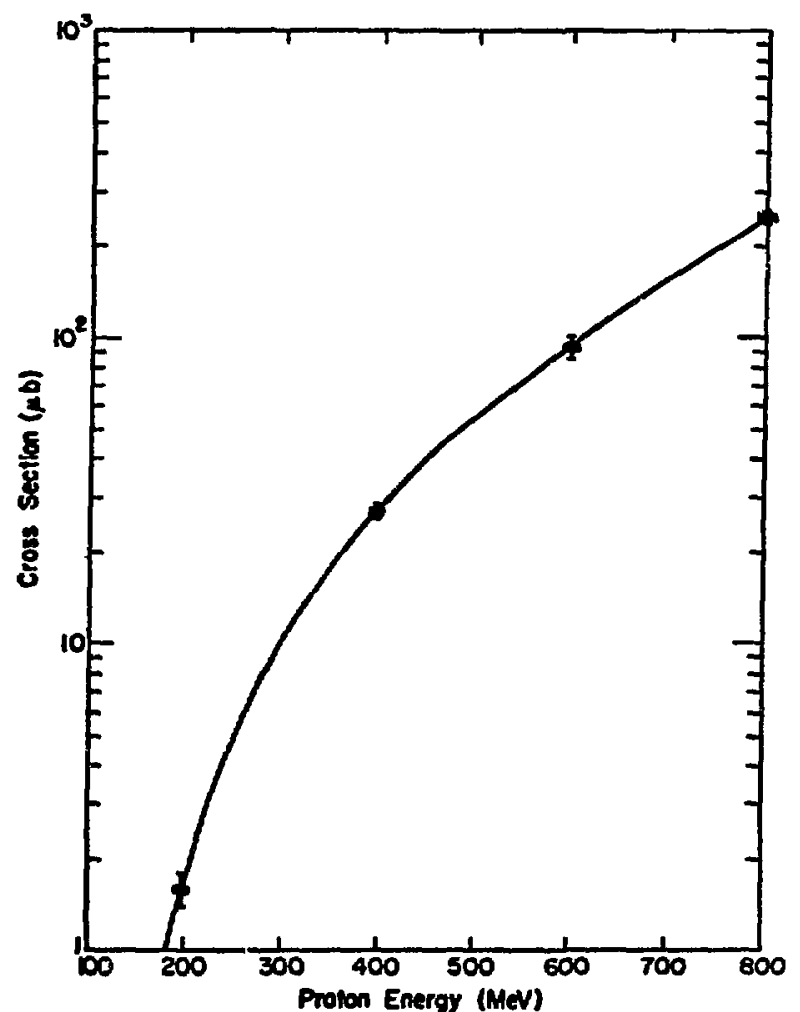

Fig. 1X-10. Cross sections for the ${ }^{197} \mathrm{Au}(\mathrm{p}, \mathrm{X})^{24} \mathrm{Na}$ reaction.
A pair of exploratory pion irradiations (190$\mathrm{MeV} \pi^{+}$on normal zirconium and molybdenum target foils) was recently carried out on the $\mathrm{p}^{3}$ channel. The experiences with these initial bombardments will be of inestimable benefit in planning and executing the more complete pion study planned for the highintensity pion beams.

Neutron Spectrum fro, $p+p \rightarrow n+\pi^{+}+p$
(Exp. 124). (Univ. New Mexico, Temple
Univ., LASL)

Experiment 124 has been set up and run in the Nucleon Physics Laboratory (NPL) neutron beam line with hydrogen in the neutron production target, although it is planned to operate in the EPB to permit detailed exploration of the forward neutron spectra as a function of angle. The collimators now installed permit measurement at $0^{\circ}, 14^{\circ}$, and $27^{\circ}$. Two major run cycles in September and October have been comisleted, the first at $0^{\circ}$ and $14^{\circ}$ and the second primarily at $27^{\circ}$.

Since this experiment uses time of flight for a direct measurement of neutron energies, a beam chopper was developed to select every sixteenth micropulse at the injector, thus giving an 80-ns interval for the measurement. This procedure would severely curtail beam intensity for all users so a method was developed to limit the chopping to the first $10 \%$ of the macropulse. The chopper has worked satisfactorily with careful tuning of a pair of rfdriten deflectors using both vertical and horizontal deflection.

During the rurs in September and October, ? 50000 neutrons have been measured at each angle from the hydrogen target. In addition, neutron spectra have been obtained from beryllium, carbon, and aluminum targets. A TOF spectrum for neutrons from hydrogen at $14^{\circ}$ is shown in Fig. IX-11, and a beryllium spectrum is shown in Fig. IX-12.

When the spectra are converted to missing-mass distributions, the effect of the $\Delta^{++}(1232-\mathrm{MeV})$ resonance is clearly in evidence at $0^{\circ}$ and $14^{\circ}$ but is great $1 y$ reduced at $27^{\circ}$, as expected. The behavior of the $\Delta^{++}$peak vs angle will be studied and the contribution of alternative pion production processes investigated in future runs in the EPB. 


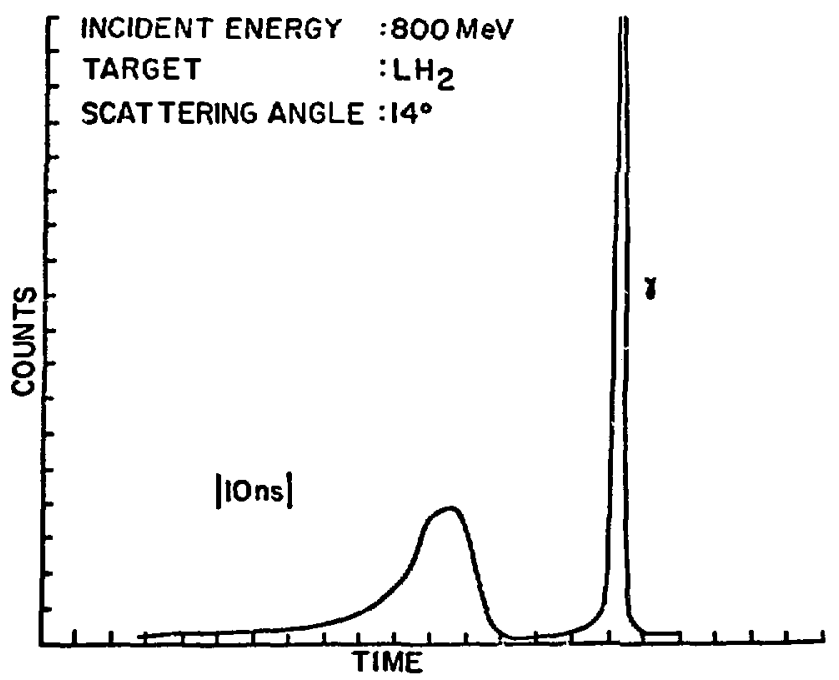

Fig. IX-11. Time-of-flight spectrum for neutral particles from the liquid-hydrogen target.

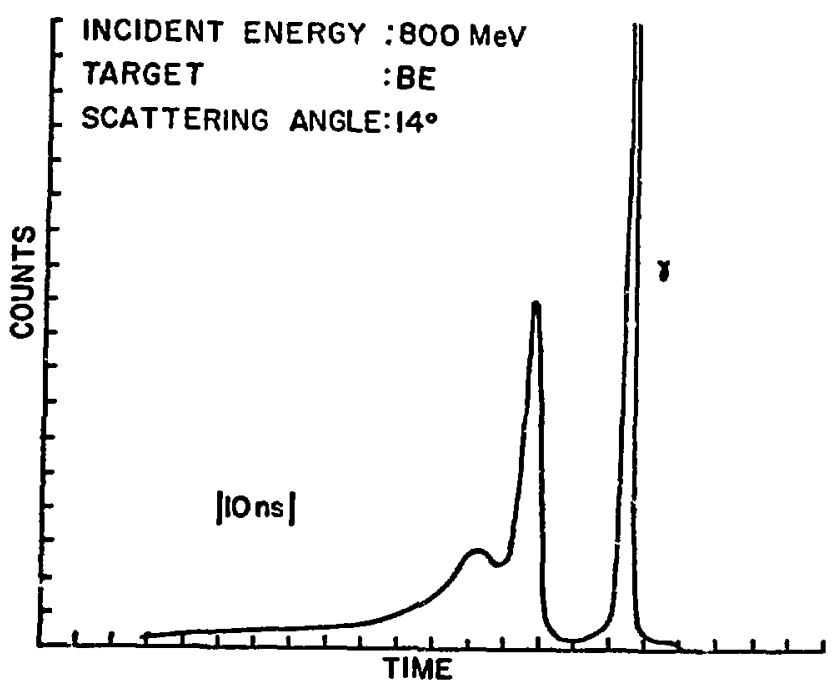

Fix. IX-12. Time-of-flight spectrum for neutral particles from a beryllium target.

The $\pi^{+}+a+p+p($ Exp. 131). Univ. of South Carolina, ORNL, Virginia Polytech. Inst., LASL)

The initial phase of data-taking forr the experiment was completed during october 1974. Angular distributions and absolute normalizations for the reaction $\pi^{+}+\mathrm{d} \rightarrow \mathrm{p}+\mathrm{p}$ were measured at pion energies of 40, 50, and $60 \mathrm{MeV}$. We intend to extend the measurement to lower energies when the accelerator beam intensity is increased.

Data at each energy were taken for 10 laboratory angles between $15^{\circ}$ and $110^{\circ}$, giving almost complete coverage in $\cos ^{2} \theta$, the appropriate parameter for this reaction. Statistics of better than $3 \%$ wer $\epsilon$ accumulated at each angular setting. Analyses of the data and of several small corrections are proceeding which will yield absolute differential cross sections with an accuracy of better than $5 \%$.

A new pion-beam monitor, utilizing the $\left(\pi^{+}, \mathrm{pp}\right)$ reaction, was installed and successfully used. It consisted of a pair of proton detectors, viewing the $\mathrm{CD}_{2}$ target at right angles to the scattering plane, with a large angular acceptance of $\sim 0.5 \mathrm{sr}$. Coincident protons were detected from $\left(\pi^{+}, \mathrm{pF}\right)$ reactions on both deuterium and carbon in the target. The monitor system worked as expected, and data were taken at the highest intensity pion beams consistent with resolution requirements.

\section{Parity Violation in $\mathrm{p}-\mathrm{p}$ Scattering [Exp.} 137 (A)]. (LASL, Univ. I1linois)

A paper has been published in Phys. Rev. Lett. giving our current result of $(1 \pm 4) \times 10^{-7}$ for the parity-violating asymetry in p-p scattering at 15 $\mathrm{MeV}$. During one run in September a preliminary result of $(4 \pm 7) \times 10^{-7}$ has been obtained for $p-d$ scattering. This result indicates a parity-violating effect two orders of magnitude smaller than reported by Lobashov for $n p \rightarrow d \gamma$. Problems encountered when using targets with a large analyzing power (carbon, deuterium) have shown the need for better polarization alignment.

Parity Violation in p-Be Scattering [Exp. 137 (B) ]. (LASL, Univ. Illinois, Univ. Chicago)

A 10-day experimental run at the ZGS has just been completed. Preliminary results 14,15 indicate that the goal of measuring the parity nonconserving part of the p-Be cross section at $6 \mathrm{GeV}$ to an accuracy of $<10^{-5}$ has been attained. A preliminary "on-line" result was obtained of $(-3 \pm 12) \times 10^{-6}$ for the parity violating effect in $\mathrm{p}-\mathrm{Be}$. As far as we know, this is the first measurement of the strength of weak hadronic interaction at high energy.

The es. veriment essentially measured the change of the transmission of a beryllium absorber as a longitudinally polarized proton beam was changed from the plus to the minus helicity state. The geometry of the detectors has been changed from that originally planned. 

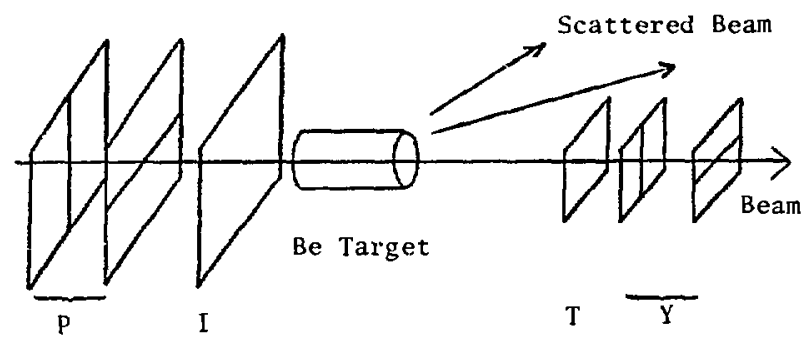

Fig. IX-13. Arrangement of detectors for parity violation experiment, $P, I, T$, and $Y$ are four scintillation detectors, split as shown, each being viewed in four directions by individual photomultipliers.

The scintillation detectors shown in Fig. IX-13 now consist of two position-sensitive detectors, $\mathrm{P}$ and $\mathrm{Y}$; an incident $\mathrm{flux}$ monitor $\mathrm{l}$; and $\mathrm{a}$ transmitted flux monitor $T$. The detectors were constructed to $0.5-\mathrm{mm}$ tolerances. The scintillators were viewed by 16 photomultipliers selected to eliminate the effects of nonlinearities and hysteresis. The anode currents were then processed by 16 voltageto-frequency converters having a linearity of $>5 x$ $10^{-4}$. These data were accumulated by an on-line computer which performed analysis and wrote the raw data on magnetic tape for future processing. The technical design goals of this system were to: 1) eliminate the effects of nonlinearities and hysteresis to the $10^{-4}$ leve $1 ; 2$ ) eliminate the effects of beam motion to the $10^{-4}$ level; 3) record sufficient data on each beam pulse to allow the further reduction of systematic errors by off-line data analysis.

The first and second goals have been attained, and the third awaits data analysis.

\section{Muon Number Conservation (Exp. 149) (State Univ. New York-Geneseo, LASL, Oregon State Univ.)}

A feasibility study of a muon number conservation experiment has been set up in the parasite beam. The chambers, magnet, electronics, and computer program for this experiment are operational; preliminary data taken in the parasite mode are being analyzed at Oregon State.
Search for Condensed Nuclear State and Study of High-Momentum-Transfer and LowEnergy-Transfer Nuclear Intcractions (Exp. 189)

(Univ. Pennsy 1vania, Temple Univ., LASL)

This experiment was proposed to LAMPF on June 14, 1974, received immediate approval to proceed in parasite status, and was set ip by July 13, following airlift of most of the apparatus from the Univ. of Pennsylvania. Since that time, a considerable volume of interesting data has been accumulated even while tune-up proceeds awaiting scheduled bean time in November and December.

The proposed experiment is to survey the region of maximum momentum transfer, e.g., $180^{\circ}$ backward scattering of protons from various nuclear targets. Topical interest to this investigation was added by the suggestion of Lee and Wick that supercondensed nuclear states mighi exist with hundreds of MeV/ nucleon binding energies. In the limit of a supercondensed nucleus with many extra neutrons, $180^{\circ}$ scattering at nearly full incident momentum would occur. The experimental scheme conceived was to use a regular LAMPF beam-bending magnet upstream from a suitable target location to bend backscattered protons out of the beam line into a detector systen. Use of a primary proton beam was considered essential to obtain adequate sensitivity.

The apparatus as set up is sketched in Fig. IX-14 showing the scintillation counter telescope adjacent to LB-BM-05. The telescope accepts positive particles backscattered from downstream targets, such as the Nucleon Physics $D_{2}$ target, at momenta determined by the telescope angle and particle charge. A special beam box, fabricated by the Univ. of Pennsylvania group, has a thin exit window to the open side of the C-magnet.

Also shown in Fig. IX-14 are three angular positions corresponding to the backscatter trajectories of protons from targets of atomic mass $2=A 2, A 4$, and A-infinity; that is, target nuclei or nuelear clusters of deuterium and helium weight and "condensons" or superheavy nuclei. The corresponding momentum of a singly charged particle is also shown. 


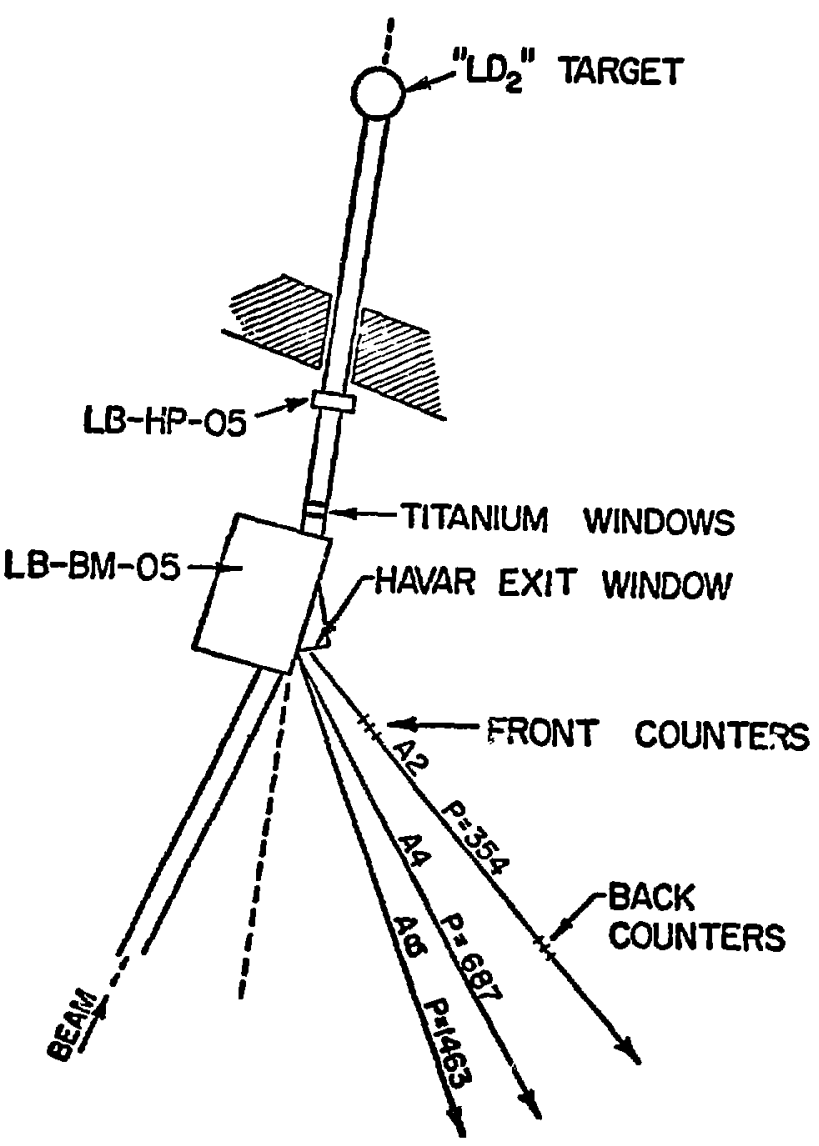

Fig. IX-14. Exp. 189 set up in the LB tunnel. Backscattered protons and other nuclear fragments are momentum dispersed by LB-BM-05 and their TOF and ionization are measured in a scintillator-counter telescope.

Multiply charged nuclear evaporation fragments will have the same trajectories for multiples of the single-charge momenta; for example, at the A4 angle, an $\mathrm{He}^{++}$fragment would have $\mathrm{P}=1374 \mathrm{MeV} / \mathrm{c}$.

Instrumentation development at present is concentrating on suppressing background at the condenson angle. The primary background is understood to be electrons converted near the apparatus. These are to be discriminated against by TOF, in an extension of the counter telescope, by their prompt arrival time vs the $201-\mathrm{MHz}$ beam $\mathrm{rf}$, by a gas Cerenkov counter signal, by removal in absorber plates, and through sweeping with a weak magnetic field

(it is assumed that the electron spectrum is soft).

Data have been taken so far at several telescope angles using three sources of backscattered particles. The obvious choice of target locations is the $L D_{2}$ location, equipped with several metallic targets as well as the hydrogen-deuterium dewar. Due to solid angle, however, the few micrometers of titanium in the vacuum windows just past LB-BM-0S provide a conparable counting rate, but unfertunately poor momentum resolution. It is planned therefore to remove these windows during the scheduled data runs. In the meantime, useful data at the lowmomenta telescope settings have been taken from backscattered particles from the titanium windows. Figure IX-15 shows the TOF spectrum over the $1.86-\pi$ telescope length at the $A 6$ angle. The peaks from left to right are electrons, protons, deuterons, and tritons. Multiply charged fragments are separated from singly charged particles of similar ToF by ionization (courter pulse height), which is also recorded for each event.

The 80-ns micropulsed beam provided for several experiments has been most useful in separating the downstream particle origins as well as improving momentum resolution by TOF.

More recently a selection of targets of our choice has been mounted on the actuat or in the LBHP-05 location (see Fig. IX-14), a preferred location for solid-angle and momentum resolution, and data have been taken with the copper target at this location and the telescope at the $A 7$ angle.

In addition to the goals initially proposed in this experiment, it is expected to be able to tie the observed cross sections for nuclear fragment evaporation to those measured at this Laboratory and elsewhere, and hopefully to extend the measurements to higher fragment energies.

Cluster Effects in Nuclear Pion Capture (Exp. 35) (Univ. Virginia, Florida State Univ.)

The 183-cm scattering chamber was set up in the ETL building and tested for vacuum leaks and mechanical performance. The experiment is now waiting to be moved to the LEP channel for preliminary runs. 


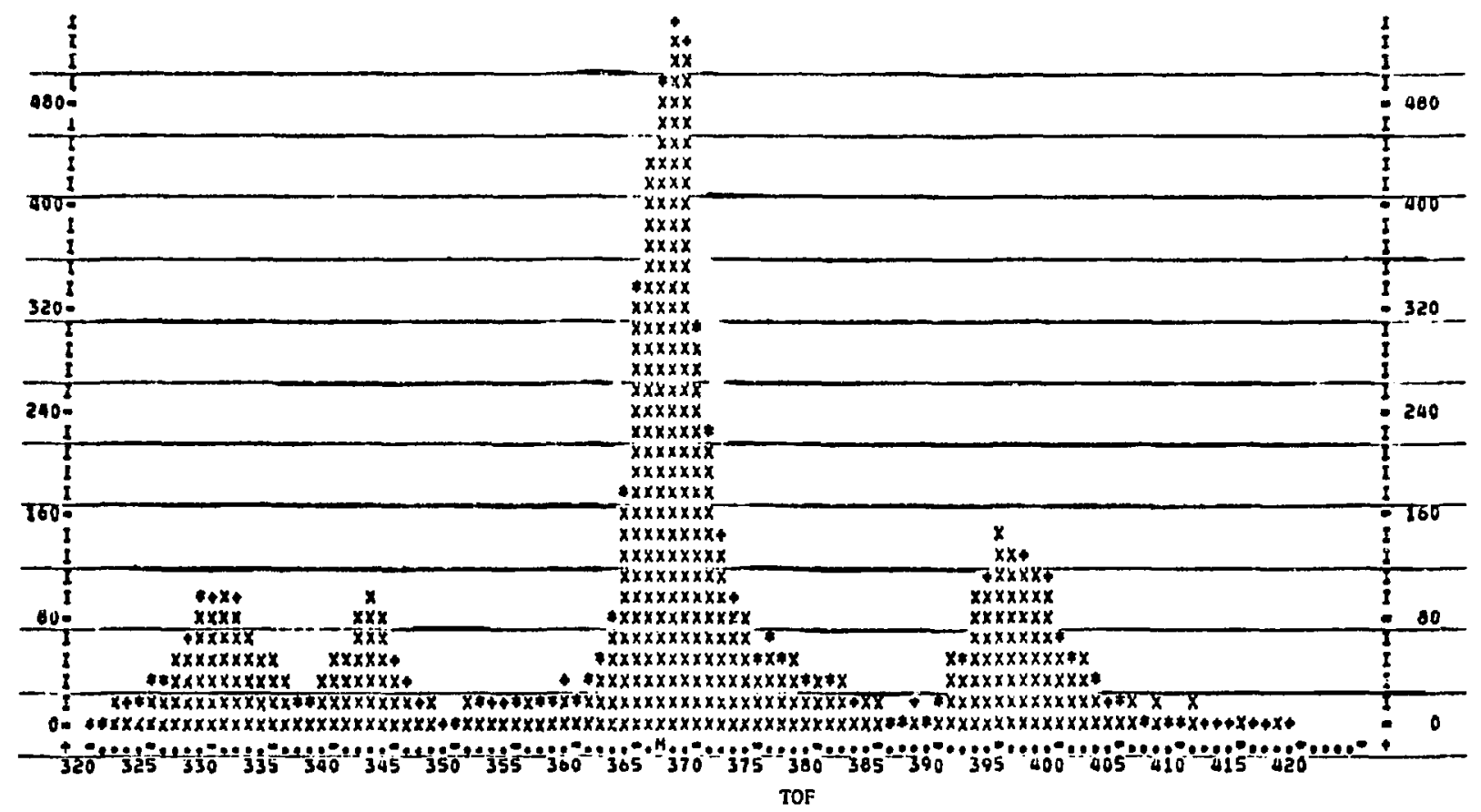

Fig. IX-15. Sample TOF data at $866 \mathrm{MeV} / \mathrm{c}$, at a magnetic deflection angle corresponding to proton backscatter from atomic mass 6 . From left to right the peaks are prompts $(\gamma-e)$, protons, deuterons, and tritons ( $\sim 15$ ns prompt-to-triton time).

Excitation Functions of Simple PionInduced Nuclear Reactions (Exp. 102) (LBL)

Excitation functions for $\pi^{+}$- and $\pi^{-}$-induced reactions through the $(3,3)$ pion-nucleon resonance on the light elements ${ }^{14} \mathrm{~N}, 160$, and $19 \mathrm{~F}$ were determined by activation techniques. The primary purpose of this study was to investigate simple pionnucleus reaction mechanisms, with particular emphasis on the $\left(\pi^{ \pm}, \pi N\right)$ reaction. The reactions ${ }^{12} \mathrm{C}\left(\pi^{ \pm}, \pi^{ \pm} n\right){ }^{11} \mathrm{C}$, for which cross sections have been accurately determined in Exp. 67, were used to monitor pion fluxes aid thus to obtain absolute reaction cross sections in this work.

Average pion fluxes of $3 \times 10^{5} / \mathrm{s}$ were found to be sufficient to study the reactions of interest. Assay of all radioactive product nuclei in this work was achieved by counting the $511-\mathrm{keV}$ annihilation radiation from $\beta^{+}$decay in coincidence using a pair of 76-x 76-mm NaI(T1) detectors. Subsequent decay curve unfolding identified the radioactive components and provided their yields. The preliminary results obtained to date lead to the following conclusions:
(1) The $(3,3)$ resonance is preserved in the excitation functions for $\left(\pi^{ \pm}, \pi n\right)$ reactions on ${ }^{14} \mathrm{~N},{ }^{16} \mathrm{O}$, and ${ }^{19} \mathrm{~F}$.

(2) The ratio of cross sections $\sigma\left(\pi^{-}, \pi^{-} n\right) /$ $\sigma\left(\pi^{+}, \pi N\right)$ at resonance ( $180 \mathrm{MeV}$ ) for all targets is $\sim 1.7$ and 1.0 as reported in prior activation studies. Both values are still at variance with the simple impulse approximation ratio of 3 .

(3) The $(3,3)$ resonance appears in the excitation functions of more complex spallation reactions, such as ${ }^{14} \mathrm{~N}\left(\pi^{ \pm}, \mathrm{x}\right){ }^{11} \mathrm{C}$.

(4) The cross sections for the ${ }^{14} \mathrm{~N}\left(\pi^{ \pm}, \pi N\right){ }^{13_{N}}$ reactions are much lower than the other $\left(\pi^{ \pm}, \pi N\right)$ reactions studied in this work, and also significantly lower than that previously reported. This observation is consistent with the instability of excited residual ${ }^{13} \mathrm{~N}$ states with respect to particle (proton) emission. 


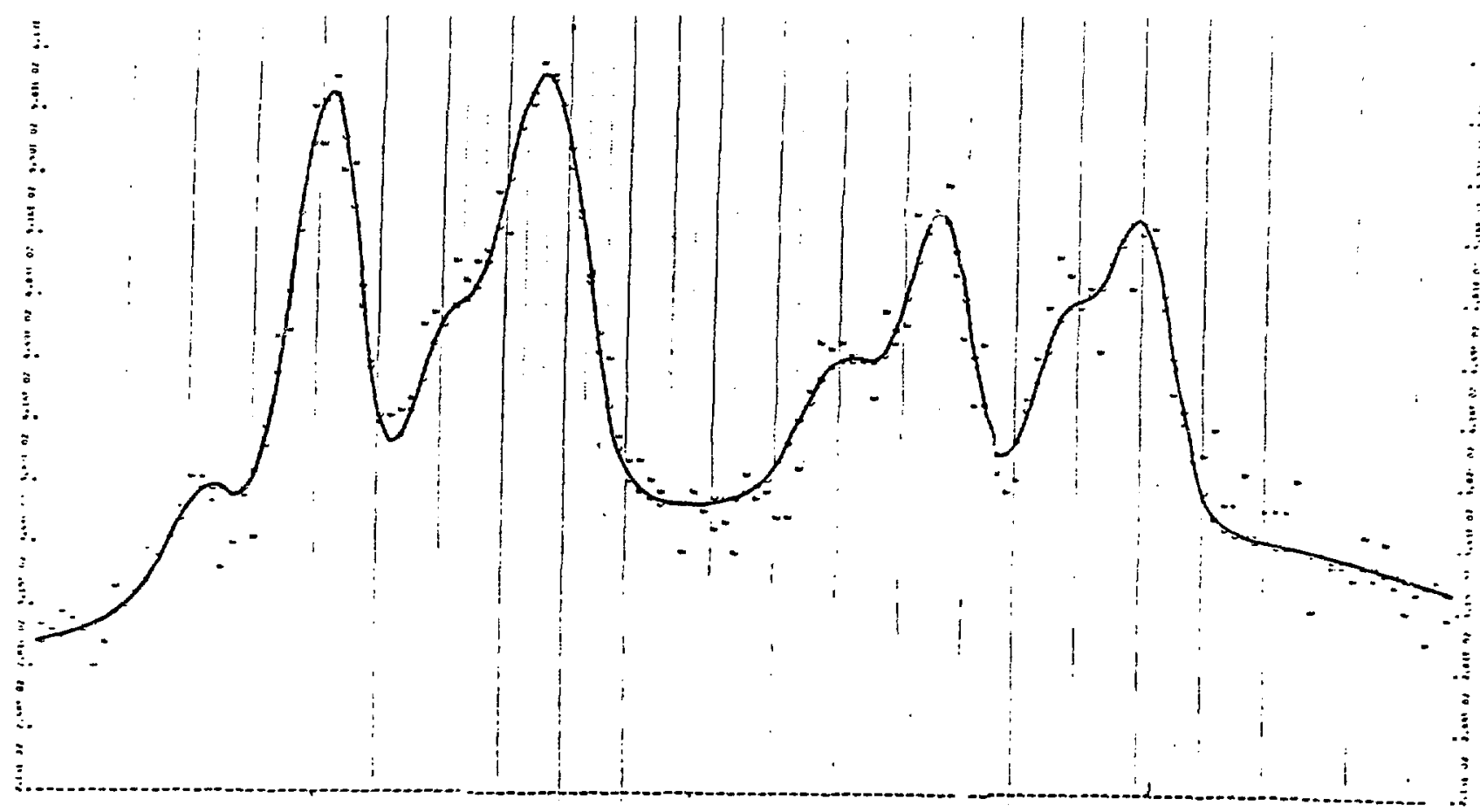

Fig. IX-16. The experimental points of the 4f-3d transition in muonic holmium, with the best-fit curve calculated from a Dirac program with a deformed Fermi charge distribution.

Precision Determination of Electric Quadrupole and Hexadecapole Moments of Deformed Nuclei Using Muonic $X$ Rays

(Exp. 166)

(Calif. Inst. Tech., Univ. Wyoming)

Measurements of muonic $x$-ray spectra of muonic atoms of ${ }^{161} \mathrm{Dy},{ }^{165} \mathrm{Ho}$, and ${ }^{181} \mathrm{Ta}$ have been taken on the west $1 \mathrm{eg}$ of the LAMPF muon channel. The energy spectra from a high-resolution $\mathrm{Ge}(\mathrm{L} i)$ counter in coincidence with the muon stop signals from a counter telescope were recorded $w i t h$ the help of the CalTech PDP $11 / 40$ system. The stopping rates were $35000 \mu^{-} / \mathrm{s}$.

The aim of the experiment was to study the hyperfine splittings of the $2 \mathrm{p}_{3 / 2}, 3 \mathrm{~d}_{3 / 2}$, and $3 \mathrm{~d}_{5 / 2}$ levels of these muonic atoms, as well as the absolute transition energies in order to explore the parameters of the nuclear charge distribution, particularly the static electric moments.

The observed hyperfine spectra were analyzed in terms of a magnetic dipole splitting, a static electric quadrupole splitting, arid a hexadecapole splitting. Figure IX-16 shows a comparison of the experimental points of the $4 f-3 d$ transition in muonic holmium, with the best-fit curve calculated from a Dirac program with a deformed Feini charge distribution. Because of vacuum polarization, Lamb shift, screening, and nuclear polarization, corrections were required.

From a fit of the $3 d_{3 / 2}$ splitting, the quadrupole moment has been obtained with an accuracy of $1 \%$. While the $3 \mathrm{~d}_{3 / 2}$ level is sensitive only to quadrupole interaction, the $3 d_{5 / 2}$ level is affected by both quadrupole as well as hexadecapole interactions. Both moments, therefore, can be derived from the data. The present measurements represent the first direct determination of nuclear hexadecapole moments with the help of muonic atoms.

Finally, a simultaneors fit with the $2 p$ hyperfine splitting (the $2 p$ level being sensitive to the nuclear charge shape) al swed us to derive the variation of the skin-thickness parameter with the polar angle $\theta$ in a nuclear coordinate system.

For ${ }^{165_{H o}}$, the static quadrupole moment appears to agree to within $1 \%$ with the moment derived from coulamb excitation assuming the rotational model of nuclei. 
Proton- and Pion-Induced Fission of Lightto-Medium Mass Nuclides (Exp. 104)

(Simon Fraser Univ.)

The purpose of the experiment is to study the fission of target nuclides covering the mass range from below silver up to uranium bombarded with protons and pions of intermediate energies. The measurement technique involves nuclear track detectors of glass and of riica, disposed in a sandwich arrangement (by which two or more fission fragments are measured in coincidence) and in single detector measurements (by which individual fragments are measured by with greater precision in regard to track length and angle of emission).

Progress has been in three general directions:

(1) Preparation for experiments: The response of individual track detectors to ions of known mass and energy has been studied in calibration experiments. Calibration of mica detectors was conducted at $\mathrm{SFU}^{16-17}$ via irradiations at the Orsay heavy ion linear accelerator; calibration of glass detectors was conducted by others entirely at Orsay. ${ }^{18}$ Additional studies have demonstrated the effectiveness of the mica sandwich technique in fission fragment spectroscopy ${ }^{19}$ while the capabilities of single mica detector measurements have been studied in a further publication. 20

Additional experiment preparation has involved the fabrication of a scattering chamber for insertion in line $B$. The necessary techniques for the preparation of thin targets for this scattering chamber have been developed and targets of $\mathrm{Ag}, \mathrm{Au}$, and $U_{A}$ for the scattering experiments are being produced at thicknesses of 100 to $400 \mu \mathrm{g} / \mathrm{cm}^{2}$.

(2) Exploratory experiments: Mica sandwiches containing targets of silver and gold were irradiated with $2 \times 10^{14}$ and $6 \times 10^{11}$ protons of 647 and $447 \mathrm{MeV}$, respectively. The purpose of the experiment was to check on the exposure needed to give satisfactory track density (track density being found in both cases to be satisfactory) and also to explore the seriousness of the background of small tracks produced by passage of the beam through the detectors, against which scanning for fission tracks must be effected. In the case of the gold irradiations, the background problems were found to be minor. In the case of the silver bombardment, the background problems were more severe. They were not, however, expected to constitute a major impediment to scanning of the micas from such experiments. A preliminary scanning of the tracks from the two experiments led to measurement respectively of average track lengths of 9-10 $\mathrm{mm}$ (approximately as expected for fragments from the symmetric fission of gold) and of 4-6 $\mu \mathrm{m}$ (likewise roughly as expected for silver symmetric fission). Fragment average kinetic energies extracted from the data are also approximately in accordance with expectation. More detailed scanning of micas from these bombardments is proceeding .

A preliminary bombardment of gold mica sandwiches with $\pi^{+}$and $\pi^{-}$over a range of energies is currently under way in a parasitic experiment. The purpose of this bombardment is to explore the same background problems as before as they may be produced by a pion bombardment.

The scattering chamber referred to above was inserted in Line $B$, with a dummy target of aluminum foil mounted in place of the $\mathrm{Ag}, \mathrm{Au}$, and $\mathrm{UF}_{4}$ targets from which data were ultimately to be taken. The assembly was irradiated with $800-\mathrm{MeV}$ protons for appropriate periods, and the target subsequently subjected to autoradiography via high-speed Polaroid film. The purpose was to explore the position of the beam spot within the scattering chamber in relation to the beam spot position recorded on a scintillating screen mounted downstream of the scattering chamber, also in Line B. In these experiments, techniques were developed for the successful alignment of the beam on the axis of the scattering chamber. It was further determined that the beam halo extended further than anticipated and resulted in irradiation of the aluminum target frames. Fission fragment production from heavy element impurities in the aluminum is not expected to be serious in the case of experiments with gold and uranium targets. Such is not the case, however, in the case of. silver bombardments, where fission cross sections are much smaller, and, in the light of this information, fabrication is proceeding of frames from high-purity graphite for the mounting of silver targets.

(3) Initiation of the irradiation program: Recently mica sandwiches containing $\mathrm{Ag}, \mathrm{Au}$, and $\mathrm{UF}_{4}$ targets were irradiated in Line $B$ with protons of $800-\mathrm{MeV}$ energy. Integrated intensities were adjusted 
to optimum values in accordance with the data obtained in the preliminary bombardments. Experiment parameters vere monitored to permit analysis of the data recorded in the detectors and the extrac$t i o n$ of the fission parameters of interest. Scanning of the detectors from these experiments will soon be started.

Search for Polyneutron Systems (Exp. 150) (Univ. Chicago, Purdue Univ.)

The summer work consisted of three preliminary stages of this experiment:

(1) Two short experiments were performed to study the high-energy halo about the main beam in Area B. Activation techniques were used. It was established that a halo (of high-energy neutrons or protons) amounting to about $0.6^{\circ}$ of the main bean was present at distances $1.6 \mathrm{~cm}$ below and above the nominal beam center.

(2) Direct irradiation of a sample of lead nitrate was performed to study background effects for the experiment. No background effect was found, a1though the sensitivity of the experiment was somewhat reduced below that expected because of melting of the lead nitrate in the direct beam.

(3) A preliminary fuli-scale run $[211 \mu \mathrm{A}-\mathrm{h})$ was performed using a boron carbide main target surrounded by lead nitrate ( $0.5 \mathrm{~kg}$ lead). The irradiation went smoothly except for a several-hour interruption. The chemical processing of the lead nitrate ran into some difficulties, partly due to the unexpected levels and types of radioactivity. The halo in this run may have been higher than indicated in the earlier study. Good experience was obtained in finding out which radioactivities were important and which chemical steps were efficient. Not very significant results $\left(\sim_{10} 0^{-6}\right)$ for the limits on hexaneutron formation can be extracted from this run.

We hope to achieve a sensitivity of approximately 100-fold better in the next run by use of larger amounts of lead detector and more efficient chemistry and counting. We are planning to use the semihot facilities in the Merrimac area to enable us to work up the radioactive detectors with less loss of time.
Investigation of In-li]ight Pion and Prcton Reactions on Nuclei (Exp. 153) (Carnegie-Mellon Univ.)

The first part of Exp. 153, the search for reaction products resulting from target bombardment with $800-\mathrm{MeV}$ protons, has been completed. Thirtyfive shifts of beam time on EPB during June and August were allocated for this purpose. Copious production of gamma rays and charged particles was observed at $90^{\circ}$ in the lab frame using the CMU dual-crystal intrinsic germanium detector system. Gamma-ray spectra were obtained from the target nuclei $\mathrm{Mg},{ }^{58 \mathrm{Ni}},{ }^{59} \mathrm{Co}, \mathrm{Y}$, and $\mathrm{Ag}$. For the first three targets, lines characteristic of nuclei lighter than the target by one nI more nucleons were observed in the spectrum. Gamma cross sections as a function of gamma energy have been obtained [e.g.,

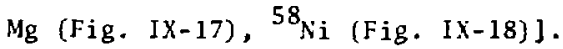

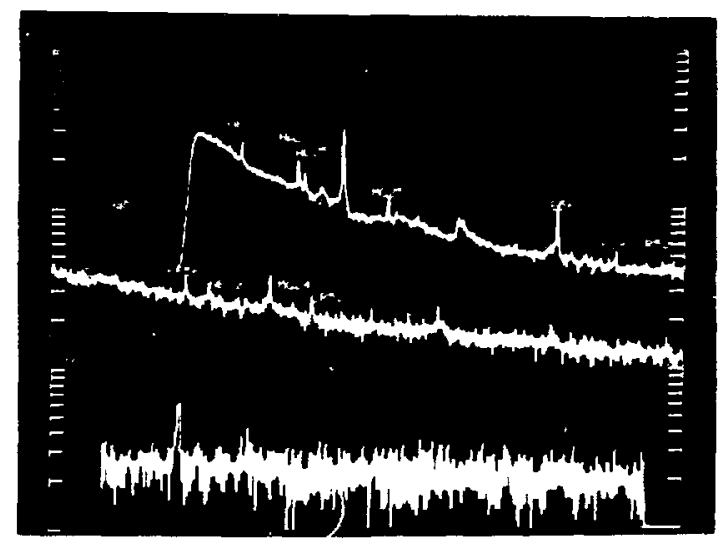

Fig. IX-17. Spectrum of $\gamma$ rays obtained when $\mathrm{Mg}$ is bombarded with $800-\mathrm{MeV}$ protons. Identifications are preliminary.

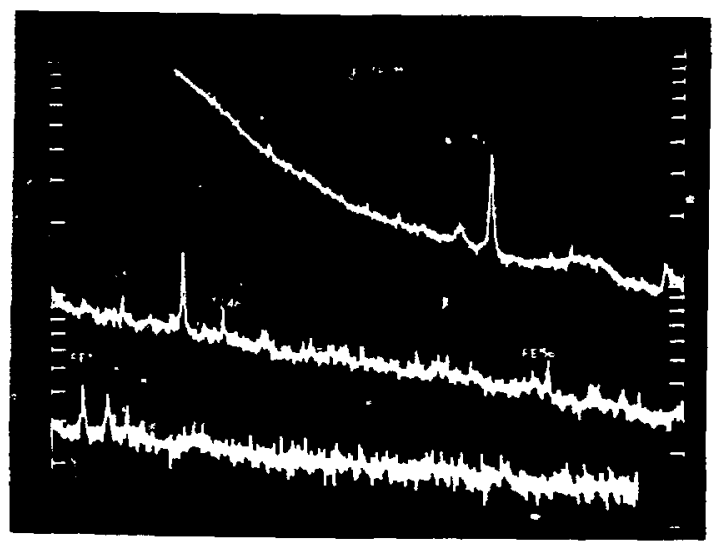

Fig. IX-18. Spectrum of $\gamma$ rays obtained when ${ }^{58} \mathrm{Ni}$ is bombarded with $800-\mathrm{MeV}$ protons. Identifications are preliminary. 


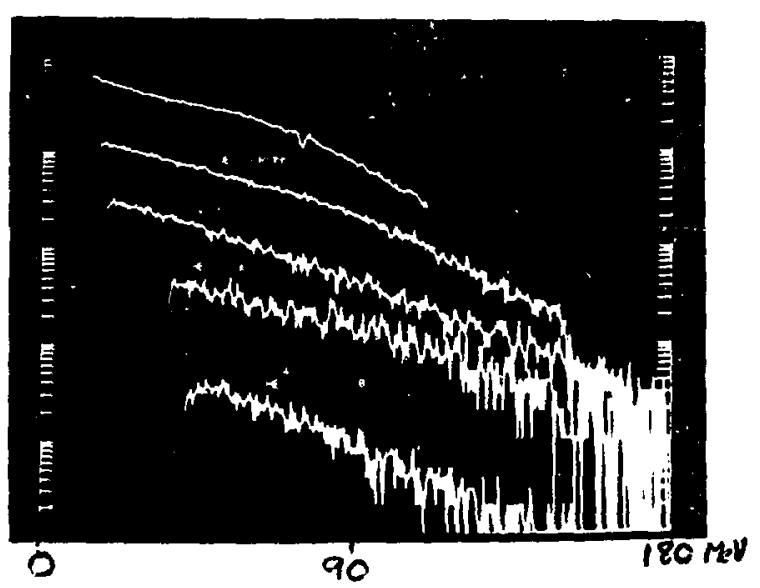

Fig. IX-19. Energy spectra of some particles emitted when $\mathrm{Ag}$ is bombarded with $800-\mathrm{MeV}$ protons.

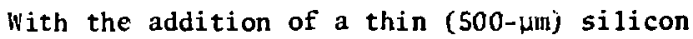
solid-state detector to the two-crystal germanium system, and operating the three detectors in a particle identification mode, unambiguous identification of protons, deuterons, tritons, ${ }^{3} \mathrm{He}$, and ${ }^{4} \mathrm{He}$ emanating from targets of $\mathrm{Mg}, \mathrm{Al}, \mathrm{Ni}, \mathrm{Co}, \mathrm{Y}, \mathrm{Ag}$ was made [e.g.. Ag (Fig. IX-19)].

For each reaction product from each target, the cross section for production at $90^{\circ} \mathrm{vs}$ particle kinetic energy has been obtained. Table IX-II below shows the range in particle kinetic energy over which the cross sections have been measured.

On the high-energy end we have been limited by the fact that the range of the various particles exceeds the stopping ability of our detector; on the low-energy side the cutoff occurs because the particle cannot travel as far as the second detector with kinetic energies below the cutoff valve.

For the $\mathrm{Ag}$ and $\mathrm{Ni}$ targets, runs at $25^{\circ}$ were also made, with varying thicknesses of copper absorber

\section{TABLE IX-II}

RANGE IN PARTICLE KINETIC ENERGY

\begin{tabular}{ccc} 
Particle & $\begin{array}{c}\text { Low E } \\
(\mathrm{MeV})\end{array}$ & $\begin{array}{c}\text { High E } \\
(\mathrm{MeV})\end{array}$ \\
\cline { 1 - 2 } & 10 & 110 \\
$\mathrm{~d}$ & 15 & 160 \\
$t$ & 19 & 190 \\
$3_{\mathrm{He}}$ & 35 & 400 \\
${ }^{4} \mathrm{He}$ & 40 & 460
\end{tabular}

between target and detector. The purpose of these runs was to obtain cross-section information at energies higher than allowed by the stand-alone detector.

Plans are under way to execute the same set of measurements for both gamma rays and charged particles using pions of $300 \mathrm{MeV}$ as incident projectiles. For chis part of the experiment, a collahoration with an Oregon State group has been arranged. They will supply a Ge(Li) detector and multichannel analyzer which will facilitate the collection of gammaray data. In addition, it is hoped to extend to lower energies the ability to measure the particleproduction cross sections.

When finished, this research will provide an interesting and useful comparison between production rates for pions and protons as fast projectiles, and will hopefully yield information about their respective interactions with nuclei.

Heavy Fragment Formation Following the
Absorption of Negative Pions in ${ }^{12} \mathrm{C}$
(Exp. 167)
(Univ. Virginia)

Following some preliminary runs during August, a six-shift data run in September yielded the raw data shown in Fig. $1 X-20$. The curves in the scatter plot (E vs TOF) correspond to (from the top) ${ }^{4} \mathrm{He}$,

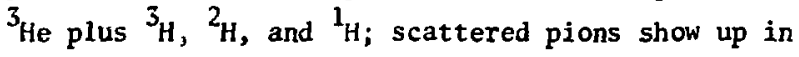
the left-hand corner. The energy was measured with a totally depleted silicon surface-barrier detector. Above a certain mass-dependent energy, the particles penetrated the detector. These events were

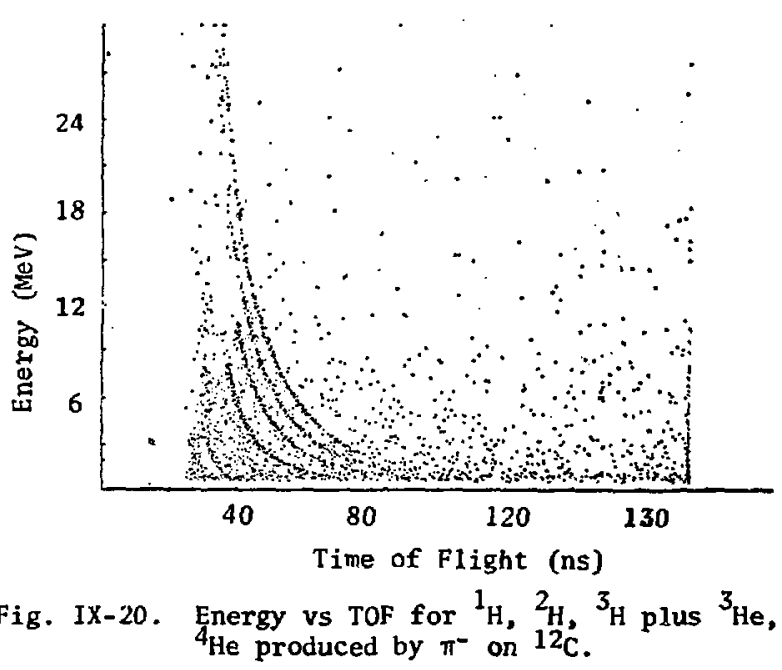


vetoed by a scintillation detector leading to the sharp upper limit in the proton and deuteron branch. In the mass-3 branch, an upper limit applying to most of the events can be seen. Above this limit, $3_{\text {He events are visible. An attempt to separate }} 3_{\mathrm{H}}$ and ${ }^{3}$ He through the insertion of a thin foil in front of the detector fell victim to an accelerator breakdown at the critical time. We are now in the process of extracting energy spectra for the individual particles from the measured distributions.

\section{Theory}

Valence Electron Orbital Distortion

Models which allow for distortion of the orbitals of valence electrons in a collision between an atom and a slow meson are being investigated. The method of Perturbed Stationary States should provide an adequate description of this distortion; however, this method is difficult to implement since it requires a complete set of electron orbitals for each meson or nucleus separation. The previously formulated photo-absorption model allows for distortion of valence electrons through the use of photo-detachment cross sections. The internal consistency of this model is being tested and its relationship to more exact methods is being studied.

Recombination between the muonic helium ion ( $\mathrm{He}^{++} \mu^{-}$) and electrons ejected during the slowing down and capture of muons in liquid helium is being investigated as a possible mechanism for enhancement of the external Auger effect and for depolarization of the muon. The probability that an ion pair will escape recombination is given by $\mathrm{P}\left(\mathbf{r}_{\mathrm{o}}, \mathrm{T}\right)=$ $\exp \left(-r_{c}(T) / r_{o}\right)$, where $r_{c}$ is the separation at which thermal energy is equal to the coulomb potential energy, and $r_{0}$ is the separation of the ion pair when thermalization at the temperature $T$ has been achieved. The thernalization radius, $r_{0}$, for electrons ejected in slowing down and capture is estimated to be 2200 mean free paths. With a total scattering cross section of $10^{-15} \mathrm{~cm}^{2}$, one obtains $r_{c} / r_{o} \sim 20$ in liquid helium at $\mathrm{T} \sim 40^{\circ} \mathrm{K}$.

To have an effect on Auger transitions in the cascade, recombination must take place on a time scale $\left\{10^{-11} \mathrm{~s}\right.$. To effect depolarization the time scale must be $\preccurlyeq 10^{-6} \mathrm{~s}$. In pure liquid, electron attachment is unlikely and in this case recombination may take place in times of the order of $10^{-10} \mathrm{~s}$.
A small concentration of impurity (e.g. $\mathrm{N}_{2}$ or $\mathrm{O}_{2}$ ) would result in a predominance of electron attachment and consequently the time scale of recombina$t i$ on would be increased to $\approx 10^{-6} \mathrm{~s}$.

The above discussion assumes that recombination occurs subsequent to thermalization. If the density of ionization is large, then the probability of prethermal recombination can be significant. Recombination by this mechanism may occur on time scales of the order $10^{-12} \mathrm{~s}$ and hence might contribute to enhancement of Auger transitions.

Bclow-Resonance Scattering of Pions from Nuclei

Work is in progress on a theory of scattering (both elastic and inelastic) of pions from nuclei for energies below resonance. A theory of the interaction of pions with nuclear matter was constructed, with unitarity included properly for pions in the medium. There are now no singularities in the theory, in contrast to some other theories. Also included were effects of the Pauli principle and finite nucleon mass; correlation effects were not included but are being investigated. The. nuclear matter results were applied to scattering from finite nuclei in the local density approximation. Semiquantitative study of elastic scattering gives reason to believe that the excess neutron distribution in ${ }^{48} \mathrm{Ca}$ can be seen by comparison of $\pi^{+}$and $\pi^{-}$cross sections. Quasi-elastic scattering provides a measure of the pion momentum in the nucleus if the recoil nucleon is detected. Other physical consequences have also been investigated, including the down-shift of the resonance.

\section{Atomic Capture of Negative Mesons}

Calculations on the atomic capture of negative mesons are continuing. Work on the Fermi-Teller model has been completed. The predicted neson distributions are remarkably impervious to changes in the atomic parameters. In contrast to previous findings some change in the distribution is found as the meson deexcites through the electron cloud.

Work on the Fuzzy Fermi-Teller model is well advanced, now including the effect of an energy gap.

Some calculations have been performed in connection with proposed experiments to detect the presence of weak neutral currents in muonic atoms. One possibility is using the asymmetry in the $3 \mathrm{~d} \rightarrow 1 \mathrm{~s}$ radiative transition for medium- and high- $z$ atoms, 
but detailed computation shows that even in the best cases the asymmetry is too small to detect at present. The most promising possibility remains the $2 \mathrm{~s} \rightarrow$ ls transition in muonic helium, but even that case does not yet appear to be feasible.

Somewhat related to this is an attempt to understand the observed disappearance of the muon polarization in helium, and the large enhancement of the external Auger rates needed to fit the observed muonic $x$-ray spectrum.

Calculations on the E2 nuclear resonance effect in pionic atoms have been performed, and several promising cases found. An experiment to detect the effect was proposed and for one case has been run successfully at the biomedical 1 ine. This constitutes the first observation of a nuclear resonance effect for hadronic atoms.

\section{References}

1. "Neutron Spectra from Proton Bombardment of Deuterium at 647 and $800 \mathrm{MeV}, " \mathrm{C}$. W. Bjork et al., Intern. Conf. on Few Body Problems in Nuclear and Particle Physics, Laval Univ., Quebec (August 1974).

2. Measurement of the $0^{\circ}$ Neutron Spectrum frum the Reaction $p+p \rightarrow n+p+\pi^{+}$at $T p=764 \mathrm{MeV}, "$ B. E. Bonner et a1., ibid.

3. "Precision Measurement of n-p Charge Exchange Cross Section at $640 \mathrm{MeV}, " \mathrm{~L}$. C. Northciiffe et a1, , ibid.

4. M. Lewis and F. Bertrand, Nucl. Phys. A196 234 (1973).

5. C. Richard-Serre et al., Nucl. Phys. B20, 413 (1970).
6. 2. Janout et al., Dubna preprint E-2726 (1966)

7. P. Shepard et a1., to be published in Phys. Rev.

8. G. Bizard et al, , contrib. to Second Aix enProvence Intern. Conf. on Elem. Particles (1973).

9. M. H. MacGregor et a1., Phys. Rev. 169, 1149 (1968) and Phys. Rev. 173, 1272 (1968).

10. P. L. Reeder and S. S. Markowitz, Phys. Rev. 133, B639 (1964).

11. D. T. Chivers, J. J. Domingo, E. M. Rimmer, R. C. Witcomb, B. W. Allardyce, and N. W. Tanner, Phys. Lett. 26B, 573 (1968).

12. M. Sternheim and R. R. Silbar, private communication.

13. R. G. Helmer et al. "Gamma-Ray Studies of the Decay of ${ }^{128} \mathrm{Ba}$ and ${ }^{128} \mathrm{Cs}$," Bull. Am. Phys. Soc. 19, 1029 (1974).

14. M. Simonium, Phys. Lett. 41B, 415 (1972); V. R. Brown, E. M. Henley, and F. R. Krejs, Phys. Rev, Lett. 30,770 (1973).

15. M. Gari, Phys. Lett. C6, 317 (1973); E. Fischbach and D. Tadic, Phys. Lett. C6, 123 (1973).

16. H. Blok, F. M. Kiely, and B. D. Pate, Nucl. Instrum. and Meth. 100, 403 (1972).

17. H. B1ok et al., Nucl. Instrum, and Meth. 119, 307 (1974).

18. M. Lecerf and J. Peter, Nuc1. Instrum. and Meth. 104,189 (1972).

19. F. M. Kiely and B. D. Pate, Nuc1. Instruin. and Meth. 109, 355 (1973).

20. H. B1ok and B. D. Pate, to be published in Nuc1. Instrum, and Meth. 


\section{$X$. PRACTICAL APPLICATIONS OF LAMPF}

(Summarizes work being performed under auspices of USAEC Division of Research, USAEC Division of Biomedical and Environmental Research, NationaI Cancer Institute, and USAEC Division of Military Application.)

\section{Radiobiology and Therapy Research Facility}

During the past quarter, major emphasis has been placed on preparing the facility for human biology trials with negative pions. The first exposures were given on October 21 . Other tasks included the maintenance of existing systems, routine checking, and equipment maintenance. All pending new hardware tasks (design and fabrication) have been itemized, along with estimated times to perform them. The list at present is estimated to require sligitly over one fear to complete with present manpower. This list will be entered on a computer disk file within the next few weeks to allow easy updating.

Specific tasks accomplished in the past quarter include expansion of the building communications system and installation of a patient communication system; installation and checkout of the 300-kVP $x-r a y$ therapy machine acquired from the U.S. Public Health Service; installation of pion flux rate meters and a computer-readable meter for recording elapsed targetin time; installation of a second TV camera in the treatment room and a TV camera and monitor for the 300-kVP $x$-ray therapy room; installation of four additional TV monitors to display computer-generated displays at the reception desk, the $300-k V P$ x-ray control panel, the conference roon, and in the treatment planning room; installation of a computer storage display terminal in the physics counting area; installation of a new $27.9-\mathrm{cm}$ electrostatic printerplotter in the control room; check out of a digital multiplexer to read the clocks and flux counters; implementation of several minor changes in the interlock system; interfacing of the graphics digitizing tablet for treatment planning; coordination of many activities for preparation of the building for human biology trials; preparation of part of a review for the final safety analysis report on the biomed facility; and assistance in establishing a PERT chart for the work planned at biomed during the accelerator shutdown effort in the first half of 1975 .
Biomedical Pion Channel Development

A permanent helium bag for the last five beamshaping quadrupoles has been designed, fabricated, and installed. A second-generation pion beam collimator has been designed and fabricated, and is currently in use for the human biology experiments. This collimator was designed with Monte Carlo calculations utilizing the measured phase-space characteristics of the biology beam. The collimator diameter can be varied from $2-5 \mathrm{~cm}$. Extensive dosimet. ric and radiobiology experiments have been carried out to determine the performance of the collimator; the results are in good agreement with predictions, and the isodose contours beneath the collimator are quite satisfactory for radiobiological applications. Data-Acquisition Computer

The MP-3 computer system is now essentially operational. Minor problems remain associated with the tape-drive interface, line printer, and area air conditioning.

Biomed Tuning

Channel tuning has continued to receive considerable effort. The behavior of the beryllium wedge is well-understood for present tunes. The combined momentum resolution of the first part of the channel and the BMO3 spectrometer is $\Delta \mathrm{p} / \mathrm{p}=1.6 \%$ (rms) which agrees well with predictions. Topics currently under experimental study include air-scattering effects, solid-angle variation with momentum and angle at the wedge, and investigation of the design fan bean tune.

The program PIFLUX was used to study how the acceptance solid angle of the pion channel and phase space of the pion beam would be affected by removing different combinations of brass shielding pieces from the bend-magnet gaps. PIFLUX showed minimal changes in the phase-space distribution and, at most, a $15 \%$ increase in the channel solid angle through the third bend if all the brass were removed.

Pion Dosimetry Program

During the last quarter, a great deal of beam time on the biomed channel has been devoted to radiobiological studies, including $\sim 50 \mathrm{~h}$ of human radiobiology (M. Kligerman, principal investigator). Much effort was expended prior to and during these experiments to insure that the needed dosimetric data were accurate and reproducible. 
The new 40-cm transmission chamber was completed, tested. and installed directly under the base plate under QN08. It is being used routinely as the primary dose monitor for all radiobiology experiments. In addition, the $20.32-\mathrm{cm}$ monitor has been installed directly under the $40-\mathrm{cm}$ chamber, and is used as a back-up monitor. Output from both monitors is integrated, and recorded by scalers. Compuzer software has been provided which monitors the output from both chambers. The target is automatically pulled after a preset dose (scaler count) is reached, or whenever the computer detects a ma1function in one of the chambers.

Using the system described above as a monitor, dosimetry was done under the new collimator with a $0.1-\mathrm{cm}^{3}$ tissue equivalent (TE) dosimeter. These rraasurements were made with the new beryllium wedge and helium bag in position and reproduced the conditions used during the radiobiology experiments.

The collimator consists of four interchangeable cones of 2-, 3-, 4-, and 5-cm diam. Representative depth-dose curves and isodose plots are shown in Fig: $x-1-6$. Peak dose rates of $0.63 \mathrm{rads} / \mathrm{min} /\lfloor\mathrm{A}-$ protons were observed under the $5-\mathrm{cm}$ cone. From these figures, it is evident that the bean is asymmetric in $x$ by several millimeters. The fact that such small deviations can be observed is due to the great improvements that have been made in chamber alignment procedures since the last report. Alignments are made by means of a pair of transits.

During the human radiobiology experiment, dose rates through the collimator had to be checked prior to each irradiation. In order to make these checks quickly and accurately, special jigs were fabricated, which fit into the bottom of the collimator. Holes were drilled in these jigs to allow accurate placement of the $0.1-\mathrm{cm}^{3}$ dosimeter. With this new systen, the entire calibration procedure takes less than

10 min. Two of the dosimeters were calibrated against a standard at Memorial Hospital, New York City, just prior to the human radiobiology runs.

\section{Linear-Energy Transfer (LET) Program}

During this last quarter, microdosimetry measurements have been continuing. Spectra have beeri taken at water depths of $2,11,17,18,75,20$, and $24 \mathrm{~g} / \mathrm{cm}^{2}$. Results are being analyzed on the CDC 7600 .
Al1 recent measurements have been taken with an improved system shown in Fig. $X-7$. The first stage of the preamplifier and all electrical connections to the Rossi chamber have been incorporated into a small Lucite cylinder to the right of the chamber. The gas leads have also been modified, and the ${ }^{241} \mathrm{Am}$ alpha source (used for caiibration purposes) has been miniaturized. This new system introduces a very minimal perturbation into the pion beam, ana is a vast improvement over the previous system. Efforts are now under way to further miniaturize this system.

The LET chamber described above has also been used to measure the neutron background in the treatment room, under conditions similar to those used

$$
\text { 2-CM COLLIMATOR }
$$

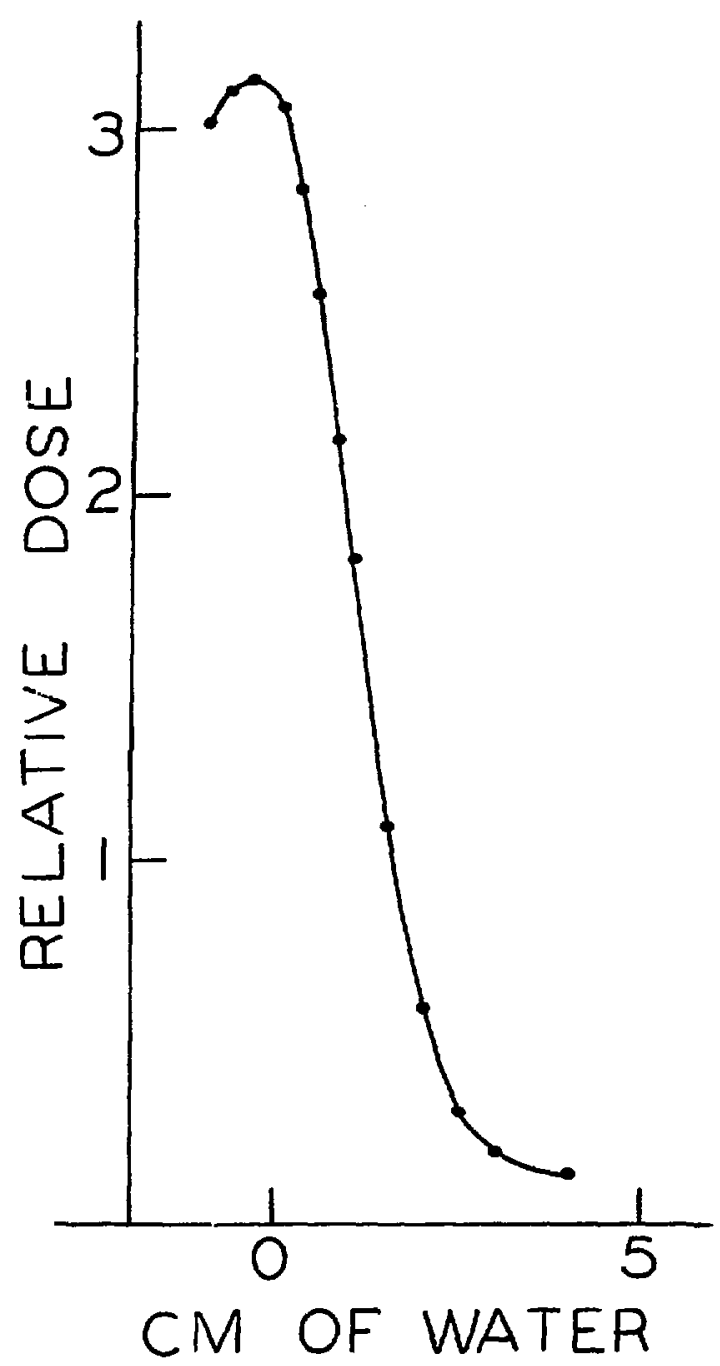

Fig. X-1, Depth-dose curve under 2-cm collimator. 
during the human radiobiology experiment. The LET chamber was used in its normal mode (ac-coupled) to measure the LET spectrum of the neutrons (and gammas), and from this the absolute neutron dose was later obtained. The neutron dose rate was found to

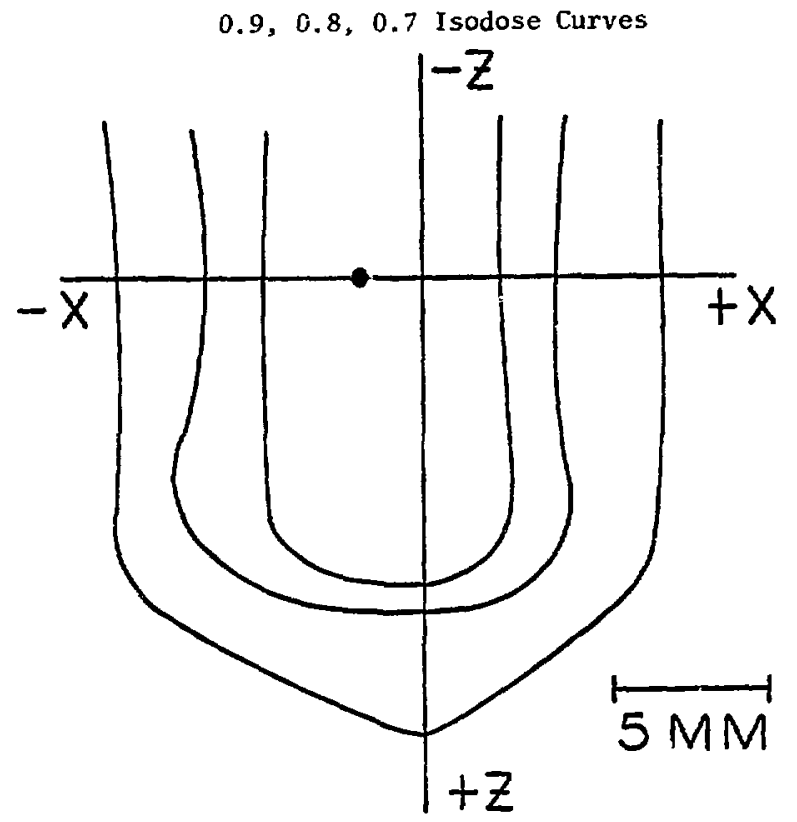

Fig. X-2, $X-2$ plot for 2-cm collimator.

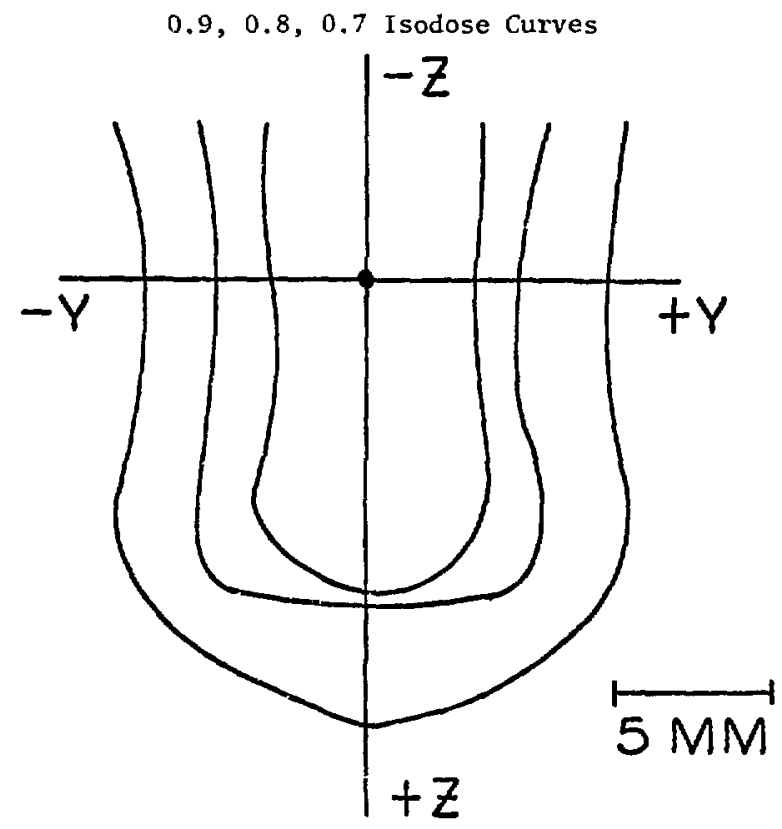

Fig. X-3. Y-Z plot for 2-cm collimator.

\section{4-CM COLLIMATOR}

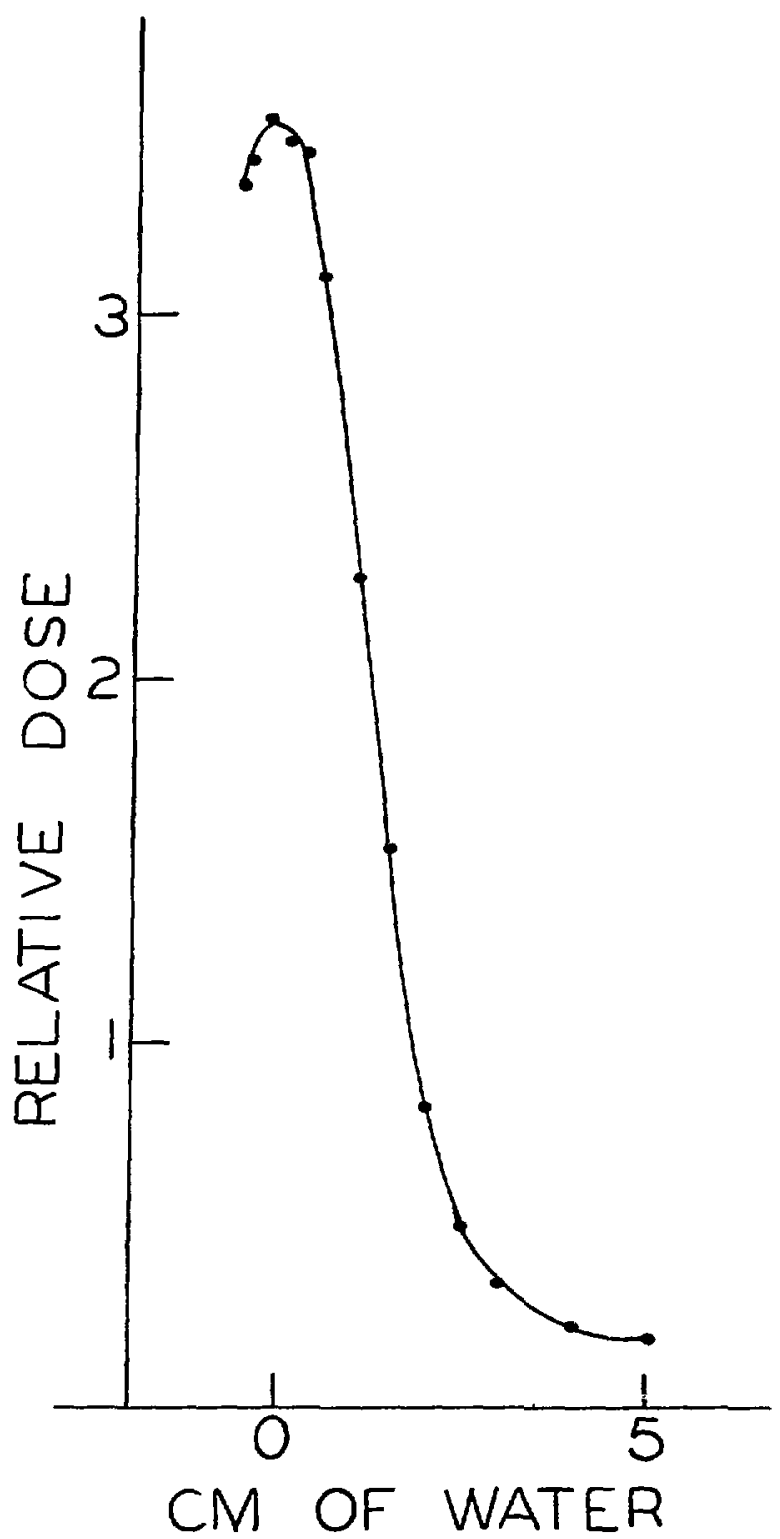

Fig. X-4. Depth dose curve under 4-cm collimator. be $30-60 \mathrm{mR} / \mathrm{h}$ at various positions in the room. The dose in phantom, at $40 \mathrm{~cm}$ from the center of the stopping region, was also measured (this geometry simulates the whole-body neutron dose to the patient] and found to be $10.8 \mathrm{mR} / \mathrm{h}$, or $0.0045 \%$ of the peak dose. These results agree quite well with measurements taken with a Bonner sphere.

Additional film dosimetry has also been done, including several exposures under the new collimator. Analysis of these films is still under way. 
$0.9,0.8,0.7$ Isodose Curves
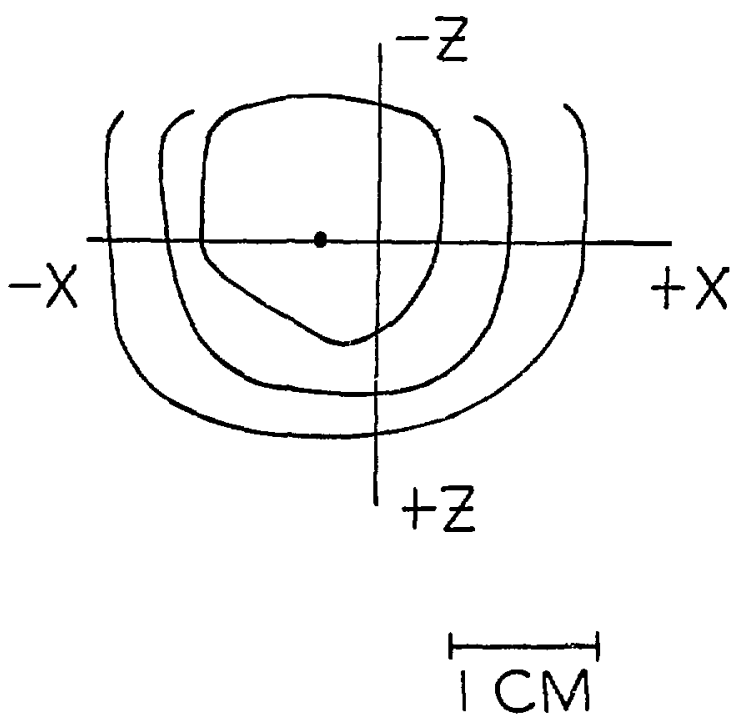

Fig. $X-5 . \quad X-2$ plot for $4-\mathrm{cm}$ collimator.
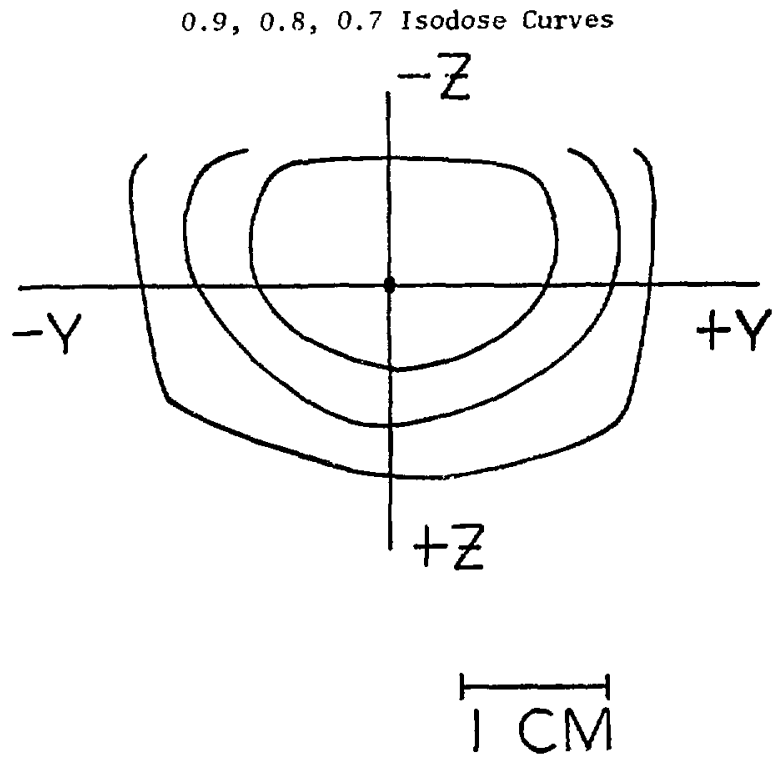

Fig. X-6. $Y-Z$ plot for $4-\mathrm{cm}$ collimator.

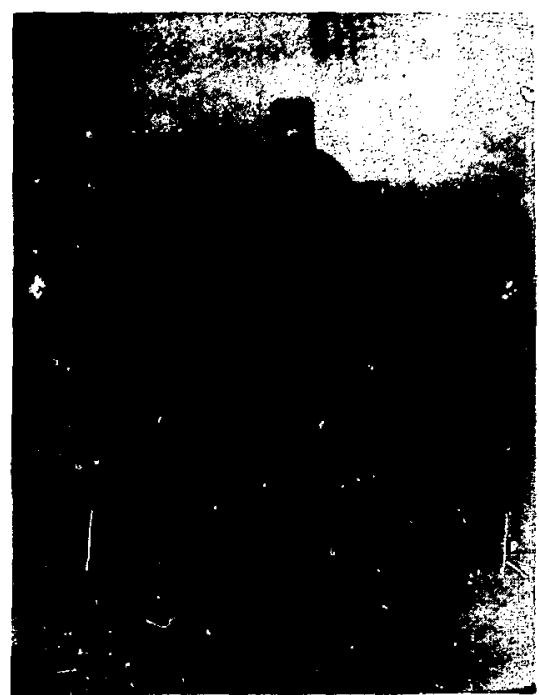

Fig. X-7. Photograph of LET chamber.

Dose Calculations

The dose due to neutrons produced in pion stars during pion radiotherapy was calculated for various locations within the human body for two idealized treatment plans using the multigroup, general-geometry, Monte Carlo transport program ANDY. The head, neck, and torso were modeled using combinations of spheres, cylinders, ellipsoids, and planes. The spinal column, rib cage, and skull were included for bone; the brain, lungs, and heart for specific organs. The neutron source was taken as the region of stopped pions with the starting position and cirection of the neutrons being randomly and uniformly distributed. The treatment volumes were chosen to illustrate rather different cases: (1) a medium-large (1-1iter sphere) tumor located in the abdominal region; and (2) a small (0.01-1iter sphere) tumor located on the left surface of the neck. In the first case, the wholebody dose was determined to be $0.8^{\circ}$ of the treatment dose; the region of the spine closest to the tumor receiving $1 \%$, and the heart and lungs $0.2 \%$ of the treatment dose. In the second case, the doses to specific sites were generally lower by 2 orders of magnitude due to the smaller treatment volume; however, the neck received $0.1 \%$ dose. These theoretical results will be checked against experiments in water phantoms being carried out at the biomed racility. 
Practical Applications Experiments

Quality of Meson Radiation Fields (Exp. 84)

Utilizing microdosimetry techniques, particlespecific LET spectra for the pion beam at the LAMPF biomedical facility are being measured. The experimental arrangement is shown schematically in Fig. $X-8$. Specific LET spectra are obtained by making LET measurements coincident with a TOF particle identification $(\pi, \mu, e)$ prior to incidence on a liquid TE phantom. A typical TOF spectrum at the pion momentum of $171 \mathrm{MeV} / \mathrm{c}$ is displayed in Fig. $X-9$. The channel tune used here is that developed for human therapy.

Figures $X-10$ and $X-11$ show pion-specific spectra which cover a range of energy deposition along the track of the particle of 0.2 to $2.0 \mathrm{keV} / \mu \mathrm{m}$ and 1.7 to $17 \mathrm{keV} / \mathrm{um}_{\mathrm{in}}$, respectively. These data were taken at the pre-Bragg peak full-width at half-maximum point in the TE phantom. When properly joined in their overlap region and combined with segments covering higher ranges of energy deposition, such sets of spectra yield a complete LET curve. The analysis of the data is being performed.

Particle-specific spectra will be measured at six points along the central axis of the therapy beam: the Bragg Peak, the two FWIM points of the peak, the plateau near the surface, the plateau near the tail of the peak; and the post peak.

Tissue Chemical Analysis with Mu-Mesic X Rays

\section{(Exp. 100)}

The apparatus for Exp. 100 was completed in June and has been used in three experimental runs since then. One more run, before the accelerator shutdown, is scheduled in November. The apparatus consists of a mon telescope comprising four scintillation counters, a muon stopping target of dimensions 8- $\times 10-\times 2-\mathrm{cm}$ thick, and a $12.5-\mathrm{cms}^{3}$ intrinsic germanium detector placed $6.5 \mathrm{~cm}$ from the center of the target. The target tissue samples are kept frozen in a thin-walled cold box.

Most of the measurements have been of mesic $x-$ ray spectra from normal tissue specimens obtained from pig and dog. Spectra have also been obtained from TE plastic, liquid, blood, and bone.

Lines from less abundant tissue elements such as phosphorus, sulfur, and potassium are not evident in most of the runs although the spectrum from a long run with TE liquid showed weak $L_{\alpha}$ and $L_{B}$ lines from potassium.

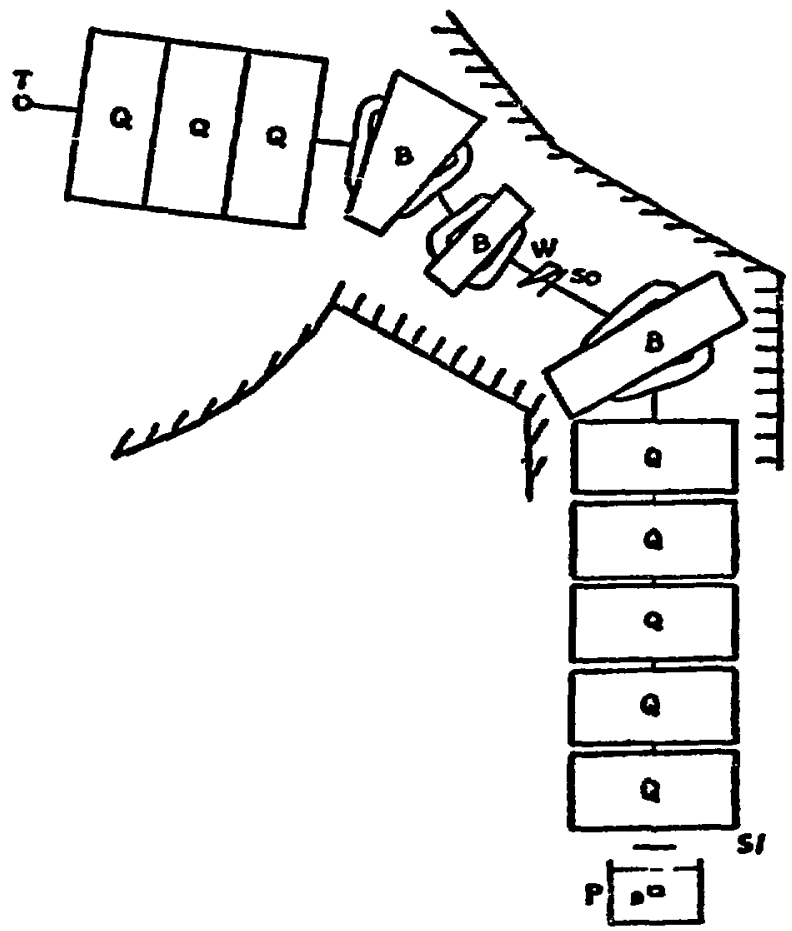

FF

Fig. $X-8$. Schematic drawing showing the arrangement of Exp. 84 in the biomedical channel. So and $S 1$ are the TOF scintillators, $P$ is the TE phantom, D is the microdosimeter.

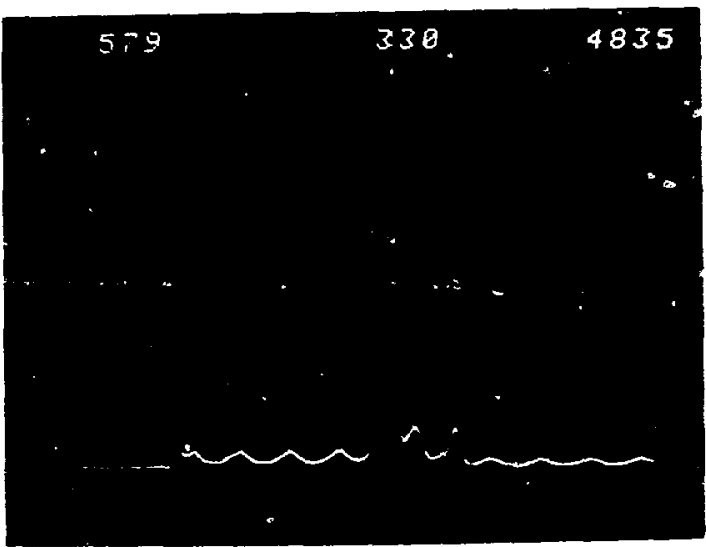

Fig. X-9. A TOF spectrum showing the $\pi, \mu$, e peaks. Time increases right to left. 


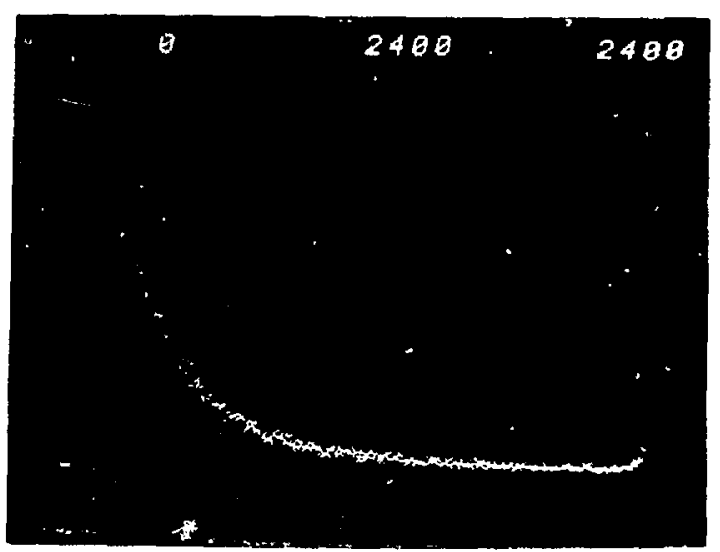

Fig. $X-10$. A pion-specific spectrum covering the range of energy deposition 0.2 to 2.0 $\mathrm{keV} / \mu \mathrm{m}$.

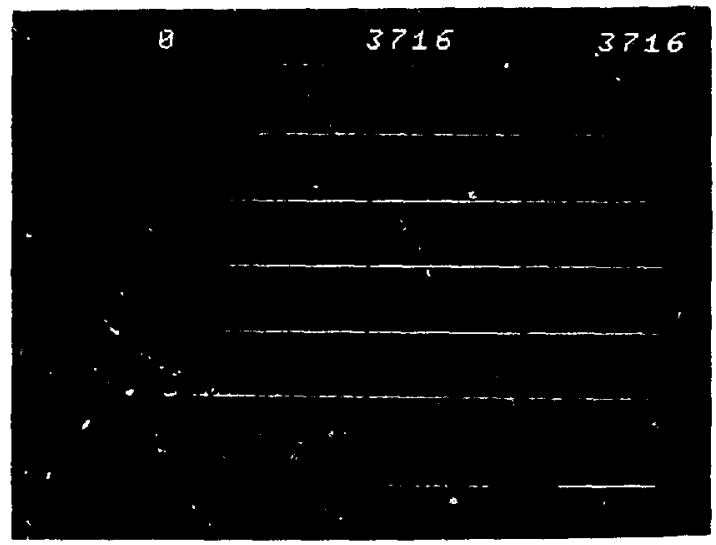

Fig. X-11. A rion-specific spectrum covering the range of energy deposition 1.7 to 17 $\mathrm{keV} / \mu \mathrm{m}$.

The data obtained so far, along with that to be obtained in November, will be used to characterize the spectra from several types of animal tissue and to gain a measure of the magnitude of the variations of line yields from one tissue specimen to another.

Analysis of the data is proceeding with most of the affort directed toward proper subtraction of background and corrections for detector efficiency and the absorption of $x$ rays in the tissue samples.

\section{Visualization of Stopping Pion Distribution} (Exp. 196)

A short experiment was performed during the last quarter to evaluate the potential of using decay products of stopping positive pions to visualize the stopping distribution. Such a method could be employed in $\pi^{-}$radiotherapy by reversing the polarities of the channel magnets before or after treatment. Positrons were detected with MWPCs and the data were computer-analyzed in real time to provide particle directions and consequently the twodimensional representation of the object in which the pions stopped. Pictures were obtained of a brass plate and a slice of a Rando phantom. The data are currently being analyzed to determine spatial resolution of the visualization technique.

Technology Transfer - Univ. of New Mexico School of Medicine and Univ. of Arizona Medical Coliege Localized RF-Current-Field (LCF) Tumor Therapy Treatment of spontaneous animal tumors is continuing at UMM School of Medicine. Only two new tumors have been treated since the last progress report: a feline squamous cell carcinoma of the nostril, and a growth, which may not be malignant, on the trunk of an elephant. Both subjects currently show no evidence of tumor and will be watched for a long period for possible recurrence. The animal referral rate has always been very uneven, and the scarcity of recent referrals probably reflects a. temporary effect. In any case, a presentation is planned for the Albuquerque Veterinary Society in December for the purpose of increasing the referral rate.

Treatment of a transplanted mouse tumor for the purpose of obtaining a thermal dose response will begin very soon. Tumor volumes will be measured by a liquid displacement technique which appears to have errors of $\sim \pm 0.02 \mathrm{~cm}^{3}$. During the past several months, a technique has been developed for homogeneous heating with LCF. The tumor (transplanted on the foot) can be heated to $42-45^{\circ} \mathrm{C}$ with a total variation in temperature $<0.5^{\circ} \mathrm{C}$ throughout the treatment volume.

\section{Differential Breast Temperature Recording} Five Temperature Difference Integrator (TDI) units are being tested by the Univ. of Arizora Dept. of Radiation Oncology. Data to date indicate that "normal" subjects may have a $\overline{\Delta \mathrm{T}}$ of at least 0 to $0.5^{\circ} \mathrm{C}$. No subjects with proven malignancy have been tested, but this should occur within a few weeks. The units now in Tucson suffer from an 
instability in electrochemical cell silver plating which was menticned in the last progress report. A new circuit design appears to minimize this effect. The new design is in the printed circuit shop and should be available for testing by December 1 .

Two hypodermic thermistor mounts were constructed here and loaned to the Univ. of Arizona for precise measurement of tissue temperatures.
Radiofrequency Tissue-Heating Calculation

The first phase of the heating calculations was completed. This phase comprised calculations for four electrode configirations:

1. Two external electrodes in a geometry similar to that used for treating near-surface implanted tumors on mice.

2. One external and one implanted electrode simulating a case in which one heats an internal organ such as the liver.

3. Two implanted electrodes with a nearby region of relatively low conductivity. This case was studied in order to determine the effect of bony regions in the vicinity of the electrodes. 


\section{MANAGEMENT}

General

Starting with this report, there will be a section devoted to management concerns, administrative policies and procedures, and information of primary interest to LAMPF users. This section will specifically address persunnel levels, budgets, safety topics, schedules and related matter, e.g., titles of internal reports and of mailings will be listed so that interested users will know about them. This will supplement but not displace the LAMPF users Newsletter.

A succinct report on the technical status of the accelerator is given in an invited paper prepared by $R$. Jameson for presentation at the IV Al1-Union National Conference to be held in Moscow in November of this year. A preprint is attached as Appendix A.

A bibliography of LAMPF-related publications is included in this report as Appendix B.

Budget

During the first four months of $F Y-75$, accrued operating costs were 4\% less than the budget forecast. However, this is a result of late deliveries rather than to lack of diligence in placing of orders.

The fiscal year financial plan was not fully funded by the AEC until late in October; approximately $40^{\circ}$ of the capital equipment allocation has been obligated or comnitted. The average number of employees--full time equivalents (FTEs)--is about $2 \%$ less than forecast at the start of the fiscal year.

Safety

With the emergence of LAMPF as a production facility, and with the increasing use of beam facilities, the safety program has expanded and become better formalized. During the past year, many new safety policies, plans, and procedures have been developed for the use and operation of LAMPF. As LANPF continues to develop and as more experience is gained the safety program will be revised and expanded to meet the new requirements. Future reports ill describe these activities and significant safeiy-related matters, including safety experience.

\section{Safety Organization}

The safety of all personnel at LAMPF, including users, continues to be of prime concern to the LAMPF administration. Since its organization in 1965, MP Division has maintained an active safety program to establish a high degree of safe practices and safety consciousness among personnel. Fig. XI-I shows the safety organization which has been developed to manage and promote the LAMPF safety program. The functions and responsibilities of the LAMPF Safety officer, LAMPF Safety Committees, and support groups are described below.

LAMPF Safety office. The LAMPF Safety office provides the focal point for all safety matters at the TA-53 site. The LAMPF Safety officer is responsible for all aspects of operational safety, physical security, and the overall safety program, including: radiological, electrical, cryogenic, and physical safety; OSHA compliance; and fire protection. For special problems, assistance can be obtained from the engineers, scientists, and safetyoriented specialists within the Laboratory. The personnel of the LAMPF Safety office includes the LAMPF Safety Officer (Assistant MP-Division Leader for Safety), the Deputy Safety officer, a part-time specialist, and a secretary.

LAMPF Safety Committee. The LAMPF Safety Committee consists of all MP division and group leaders, and senior representatives of associated LASL groups. The LAMPF Safety officer serves as chairman of the committee. The committee determines the general sa sety policy for LAMPF, reviews special problems, and establishes procedures concerning all phases of the LAMPF safety program.

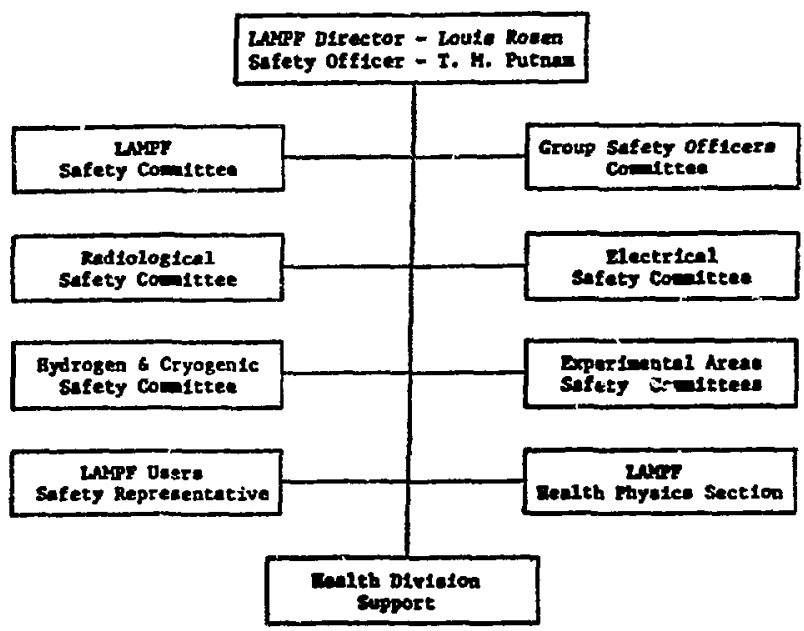

Fig. XI-1. LAMPF Safety Organization. 
LAMPF Radiological Sriety Comnittee. The LAMPF Radiological Safety Comnittee has the responsibility for coordination and supervision of radiological safety operations and for the review and development of radiation control and safety procedures.

LAMPF Hydrogen and Cryogenic Systems Safety Committee. The LAMPF Hydrogen and Cryogenic Systems Safety Conmittee is vesponsible for the safety aspects, on-site review, operational coordination, and supervision of hydrogen and cryogenic systems. This committee establishes written general procedures and guidelines for the use of hydrogen in the experimental areas of TA-53. Also, the committee reviews each experiment in which the use of hydrogen and/or a cryogenic system is proposed. This review includes design, adequacy of operating procedures, and safety protection.

LAMPF Group Safety officers Committee. The Group Safety officers Comittee has been established to further strengthen the LAMPF safety program, to provide training in specific topics, and to encourage discussions of safety problems. Each MP-Division group and each group with a significant program at LAMPF have appointed group safety officers to serve as members of this committee. Their primary responsibilities are to assist the group leader in carrying out his responsibilities for safety within the group's operation, and to maintain and promote a high degree of safe practices and safety consciousness among the group's personnel, and assist in the safety training of new personnel.

LAMPF Electrical Safety Committee. The nature of the LAMPF accelerator and its varied research activities expose personnel to many combinations of electrical equipment and unique applications. Consequently, extra efforts are required to minimize the risks encountered at LAMPF by assuring that adequate safety measures are developed and followed in the design, construction, operation, and maintenance of the vast amounts of electrical equipment. The LAMPF Electrical Safety Comittee has been established to continually review the adequacy of electrical safety measures provided throughout the pruject. It is responsible for reviewing and updating the Electrical Safety Guides, for reviewing and recommending changes in electrical safety procedures, and for coordinating and supervising the electrical safety aspects of the LAMPF safety program.

LAMPF Users Safety Represiniacive. The LAMPF Users Safety Representative is appointed by the Chairman of the LAMPF Users Group. He serves as a member of the LASPF Safety Committee and the LAMPF Group Safety officers Comnittee and provides contiauity between experimentalists and the LAMPF Safety office.

\section{Safety Procedures}

Considerable effort was spent this quarter on the following written safety procedures.

LAMPF Radiological Safety Procedures. The LAMPF Radiological Safety Procedures were completely revised to reflect operating experience and current operating procedures. Two changes in policy have been made which will aid in limiting personnel exDosures to the Jowest limits practicable:

1. A written operational plan is required before beginnisg any work in which the total exposure to personnel is reasonab!y expected to approach 0.3 man-rem, and any operation involving personnel entry into any target-cell or beam-stop area. This written plan must include a detailed plan of the operation, use of special equipment and tools, expected time involved, and predicted exposure. Fifteen plans have been prepared and used since the beginning of the quarter, with significant reductions in personnel exposures.

2. No person is exempt from working in radiation areas on tasks he is qualified to perform. This will spread radiation exposures among all qualified personnel.

Personnel Safety System Procedures. Procedures for operation of the personnel safety systems for the Low-Energy Pion (LEP), Stopped Mtuon Channel (SMC), and High-Energy Physics $\left(P^{3}\right)$ channels were prepared. These procedures specify steps that must be taken to clear personnel from a channe? and secure its personnel safety system, and to make routine or restricted entries while permitting uninterrupted bean to other Line A channels. These procedures reflect the philosophy that for routine entry to a channel a minimum of two independent methods of removing beam from the channel shall be used, e.8.: inserting two beam plugs, inserting a 
beam plug and adjusting a magnet to deriect the beam, etc. With present accelerator operating conditions, restricted personnel entry is permitted into certain channels during channel operation. The new procedures specify the precautions and special actions to be taken for such entries.

Standard for Magnet Warning Lights. After studying a number of magnet warning-light techniques and the electrical circuits to activate these lights, a standard was chosen. A red light on each magnet will be activated when the magnet power supply is turned on by actuating a $24-V$ de relay with the $24-V$ dc control voltage that operates the contactor for turning on the magnet power supply. When the relay is actuated, 110-V ac are applied to the $1 \mathrm{ight}$ through the relay contacts. The 110-V ac supply will be hard-wired from a breaker to the relay contacts, with no intervening disconnects. A neon light mounted below the warning light indicates the presence of $110-V$ ac for actuating the warning light. The warning 1 ight will be 100-W, 220-V ac and, being operated at $110 \%$, should last indefinitcly. The system is not completely fail-safe, e.g., the warning light can be burned out, the relay can fail, etc., leading to a situation where the warning light can be out and the power supply on. However, the nurpose of the light is not to indicate that it is safe to begin work on, or very close to, the magnet. Standard lockout and tag procedures are to be used before beginning such work. Because all magnet terminals will be covered, no direct electrical hazard should be present. Thus, it is concluded that the system will provide an adequate margin of safety in alerting knowledgeable personnel as to the on/off status of magnet poker supplies, with a reasonable degree of confidence.

New magnet systems will be equipped to conform to the standard, and existing systens will be changed as time and funding permit.

Tritium Experiment. An experiment was conducted in the LEP-East channel to study radiative pion capture in light nuclei. This required special safery considerations because of the large guantity ( $80000 \mathrm{Ci}$ ) of $L T_{2}$ used as a target. The steps taken to minimize possible hazards are described below:

An enclosure equipped with a forced exhaust system wis constructed for the entirs tritium- containing apparatus, including the target cell. The exhaust duct was vented above the roof of Building $A$ and contained tritium detectors to provide early leak indication. Tritium detectors were also placed at selected occupied locations in the experimental areas.

Detailed written procedures were developed that specified the operation of the LT? target dewar system, operational restrictions and regulations, personnel access and control, physical surveillance, etc. Energency procetures covered sjtuations involving potential or actual release of tritium.

Personnel directly involved in the operation of the tritium apparatus were trained in the use of plastic suits for entry to the enclosure in case of an emergericy.

An information session was held for personnel whose tork normally required them to be in the experimental areas. Contractor personnel were briefed by their supervisors on emergency procedures.

Training. The use of fire control equipment was demonstrated by personnel from the Los Alamos Fire Department at a divisionaride safety meeting.

First-aid classes are being conducted for all interested personnel. Fifteen have completed the course this quarter and more than fifty others are scheduled for training.

Personnel Levels

During this quarter, 41 full-or part-time personnel joined MP Division, while 19 terminations or transfers took place, bringing the number of FTE employees in MP Division, as of October 31, 1974, to 319. Seven of these employees are long-term visiting staff members; three of the terminations this quarter were staff in this category.

Guests

luring this report period 120 users vere received at LAMPF and 153 users weze checked out. Currently, there are 75 users at LAMPF. In addition, there are more than 50 guests on long-term visits to the facility.

Among the services that are provided to LANPF: guests are the following:

1. Secretarial and typing services.

2. Travel arrangements, and housing and lodging reservations.

3. Housekecping services are provided for those running expuriment: and using on-site overnight accommodations.

4. Guest mail center setvices. 
5. Stationery stock and photographic darkroom are maintained.

6. Scheduling for after-hours terminal operators for 7600 computer.

7. Liaison activities between LAMPF guests and other LASL support offices for such items as reimbursement for travel, subsistence, fecs, etc.; security clearances, if required; $v$ isits by non-U.S. nationals; medical services; library services.

LAMPF USERS GROUP, INC.

\section{Liaison Office}

The annual election of the Board of Directors of the LAyp!: Isers Group. Inc, was conducted by mail ballots sent to the 1021 members of the group on September 23, 1974. A lominating Comnittee consisting of Kenneth $\%$. Crowe (LBL), Chairman; Peter Carruthers (1ASL); Paui Todd (Pennsylvania State Univ.); Id Y. llungerford (Univ. of llouston); and Harvey B. Hillard (Case liestern Reserve Univ.) prepared a slate of candidates. In additional candidatc was placed on the ballot by petition. Following is the slate of candidates:

Chairman-lilect :

David A. l.ind, Univ. of Colorado

Bosrd of lirectors:

Lewis Agnew, LASt

Joln C. M1red, llniv. of llouston

kapel $x$. Seth, Northwestern Iniv.

The two candidates for Board of Directors receiving the most votes will replace Pohert J. :lacek (LSL) and Stanley !:. Sohottka (Univ. of V'irginia), who are completinf, their two-yenr term of office. The leasline for the retur: of ballots to the Liaison office is Yovember 4, 1974. Results of the balloting will be announced at the Eighth LA!PF Users iseting.

Preparations for the lighth JANll: lisers iketing to be held at Los Alamos on Yovember 11-12, 1074, are being made by cluaiman lemon t:. llughes (Yale Iniv.) and the liaison office. Prelininary prograns, reservations forms, and other meeting, information were mailed to ali memers on bejtenher 23, 1974. The progran will consist of temarks by Richard F. Taschek, LASl. Associate birector for lescarch; LuIPF Status Reports by Louis Rosen, Director of LA:Il: and bonald C. lagerman, Associate Division
Leader for Operations; Annual Users Group Report by Vernos $H$. Hughes; a general business session; and reports on results of LAIPF experiments. Invited talks are scheduled by Moger llildebrand, Univ. of Chicago, "New Opportunities in lledium Energy Science"; Erncst ?. Henley, Iniv, of Hashington, "Parity Violations"; Alfred S. Goldhaber, State Univ. of :ew York, "Relativistic lleavy Ions"; and 11.-J. Gerber, ETH and SIX, "Report on Research at SI:." In addition, all Working Groups will hold meetings on Tuesday afternoon, :iovember 12, except the Bionedical Horking. Group, which plans an all-day meeting starting at $8: 30 \mathrm{a} . \mathrm{m}$. The Tumor Localization Subcommittce of the Bionedical Steering Committee plans to hold its meeting on November 10-11, 1974. A reception at the Community Building followed by a banquet at the Los Alamos Inn will be held Monday evening, November 11 . The banquet speaker will be Stirling A. Colgate, President of lew lexico Inst. of tining and Tech., who will speak on "Scientific Literacy and Astrophysics."

The sotice of innual yeeting and call for Sominations and Amendnents to By-Laws, mailed August 3 , and the 1975 membership-renewal forms, mailed Septemier 25, were sent to the entire membership of the LUMPF Users Group, Inc., in compliance with the by-laws. A ballot for the election of Chaiman of the LAtIPF Bionedical Users Steering Committee was prepared and miled to the biomedical users.

The LAMF Users llandbook was completed and sent to press. It is expected to be ready for distribution carly in Sovember.

\section{Board of Directors}

The Board of Directors of the LUMPF Users Group, Inc., will meet at convenient times during the LNIPF Users keting. The newly elected officers for 1975 will be invited to attend these meetings. Agenda itens include the following: 1) appointments to the Cryogenics Targets Subcomnittee, 2) piceures of LNIPF users, 3) taxi service for LAMPF users, 4) telephones in the LASL-adninistered kitchenette apartments during the Great Shutdown, 5) membership or institution fees, and 6$)$ set date for next Board meeting (January or February 1975). 
Technical Advisory Panel (TAP)

The Teclinical Advisory Panel of the LAMPF Users Group, Inc., will hold its mecting on November 13, 1974. The three main agenda items will be: 1) a report from Morton M. Kligerman, M.D. (IASL/Univ. of New llexico School of Medicine) on the radiotherapy treatment program and its effect on the LAMPF facilities, 2) discussions of the priorities associated with the FY-75 budget, and 3) status report on the EPICS and IIRS channels.

The New Facilities Subcommittee of the TAP, chaired by Stanley E. Sobottka (Univ. of Virginia), will hold a meeting in Los Alamos on November 8 to discuss progress on the EPICS and HRS channels.

\section{Statistics}

Listed below are statistics of the LNIPF Users Group, Inc., as of nctober 1, 1974.

Tembership

:Ton-LASL

LASL

Total

1026

Fields of Interest

1. Vuclear and Particle Physics 76 ?

2. Nuclear Chemistry 173

3. Bionedical Applications 368

4. Yot Specified

5. nther (including administration, facilities, operations, coordination, theory, data acquisition, and miscellaneous applications)

6. Isotope Production 120

7. lladiation Damage 132

8. Weapons lieutron Research 113

(Votc: These numbers do not add to total menibcrship because there are duplicate interests.)
Institutional Distribution

1. Membership by Institute

LASL

nther National or Government

Laboratories

U. S. Universities

421

Industrial

Foreign

llospitals and Iledical Centers

$\underline{102}$

Total

1026

2. Number of Institutions

National or Government Labs

IJ. S. Universities

112

Indus try

Foreign

Hospitals and Medical Centers

Total

Regional Breakdown

East (PA, :IJ, DE, Wash DC, MA, :YY, CT, VT, RI, :H, MF)

Midwest (OH, MN, KS, IN, WI, M1, IL, ND, SD, NE, IA, It

South (MD, VA, T:S, AR, IW, KY, NC, $A L,:(S, L \Lambda, C A, F L)$

Soutȟwest-Mountain (AT, ID, IT, WY, $A Z, C O, M, O K, T X)$

Far liest (AK, HI, NV, WA, OR, CA) 136

Foreign

IASL 
Experimental Program

Operational Experience

During this quarter 39 different experiments received bean for a total of 1058 eight-hour shifts and a total of $30729 \mu \mathrm{A}-\mathrm{h}$. The beam supplied to each experiment is given in Table XI-I. This tabulation does not include parasite use of beam during production nor does it include the beam used in facility development activities. Experiments 26,165, and 175 have been completed.

TABLE XI-I

QUARTERLY REPORT OF BEAM PROVINED FOR FXPERIMENTS

\begin{tabular}{|c|c|c|c|c|c|c|c|}
\hline $\begin{array}{c}\text { Proposal } \\
\text { No. } \\
\end{array}$ & Channel & $\begin{array}{l}\text { Shifts } \\
(8 \mathrm{~h})\end{array}$ & $\mu \mathrm{A} \mathrm{h}$ & $\begin{array}{c}\text { Proposal } \\
\text { No. } \\
\end{array}$ & Channe I & $\begin{array}{l}\text { Shifts } \\
(\mathrm{Bh})\end{array}$ & $\mu A h$ \\
\hline 25 & LEP & 11 & 470 & 111 & BSA-RAD & 10 & 389 \\
\hline 27 & EPB & 50.5 & & 118 & SWY-LABS & & 0.5 \\
\hline 32 & $P^{3}-W$ & 48 & 1897 & & $A B-N u c c h e m$ & 0.1 & \\
\hline 34 & $\mathrm{P}^{3} . \mathrm{E}$ & 65.5 & 3426 & 123 & $p^{3}$ & 2 & 122 \\
\hline 37 & SMC-S & 80.5 & 3870 & & AB-Nucchem & 0.75 & 2.2 \\
\hline 44 & Biomed & 4 & 162 & 124 & AB-Nucchem & 29.5 & 70 \\
\hline 56 & AB-Neutrons & 32 & 102 & 125 & $A B-N e u t r o n$ & 25 & 105 \\
\hline 60 & $S M C-H$ & 17.5 & 848 & 131 & LEP & 18.5 & 1126 \\
\hline \multirow[t]{2}{*}{67} & $\mathbf{p}^{3}-E$ & 16.5 & 813 & $14 \hat{3}$ & Biomed & 109.5 & 3137 \\
\hline & LEP & 11 & 635 & 148 & Neutrino-A & 35 & 1072 \\
\hline 81 & EPB & 69 & & 150 & AB-Nucchem & 1.5 & 12 \\
\hline 84 & Biomed & 4 & 90 & 151 & Biomed & 1 & 32 \\
\hline 86 & TTA & 60 & 2992 & 153 & EPB & 16 & 1 \\
\hline 96 & LEP & 85 & 5016 & 161 & BSA-RAD & 15 & 552 \\
\hline 99 & $p^{3}-w$ & 12 & 660 & 163 & SMC-K & 21 & 847 \\
\hline 100 & $\operatorname{SMC}-W$ & 36 & 834 & 166 & SMC-W & 13.5 & 631 \\
\hline \multirow[t]{2}{*}{102} & LEP & 0.5 & & $16 ?$ & Biomed & 11 & 411 \\
\hline & $\mathrm{p}^{3}$ & 0.6 & 0.9 & 174 & BSA-RAD & 1 & 23 \\
\hline 103 & $p^{3}-E$ & 0.3 & 23 & 189 & $A B$-Neutrons & 37 & 139 \\
\hline 104 & AB-Nucchem & 0.5 & 0.8 & 195 & Biomed & 3 & 104 \\
\hline 105 & AB-Nucchem & 2.5 & 17.5 & 196 & Biomed & 3 & 85 \\
\hline 105 & AB-Nucchem & 0.85 & 4.9 & & & & \\
\hline
\end{tabular}




\title{
APPENDIX A
}

LAMPF PROTON LINAC PERFORMANCE

\author{
by \\ Robert A. Jameson \\ Los Alamos Scientific Laboratory \\ University of California \\ Los Alamos, New Mexico, USA 87544
}

-

\begin{abstract}
The present status of work directed toward bringing the LAMPF proton linear accelerator to design intensity and accelerating both $\mathrm{H}^{+}$and $\mathrm{H}^{-}$leams simultaneously is described. An overview of the performance is given, and then the transverse and longitudinal phase space investigations are covered in more detail, covering the techniques used for measurement, the results to date, and discussion of the work remaining. The program for measuring and impirving longterm stability and reliability of the accelerator facility is briefly described.
\end{abstract}

\section{INTRODUCTION}

Since completion of construction of the LAMPF proton linac and its initial commissioning at 800 $\mathrm{MeV}$ in June 1972, much of our effort has concentrated on completion of the experimental areas and beginning the experimental program. One reason for this approach was o allow all aspects of the facility to then share in the leaming that would accompany the increase in beam intensity toward the design goal. The beam switchyard became operational in late 1973, and a broad experimentai program began in early 1974 . At present, the shielding in the experimental area 1 imits production operation to $10 \mathrm{HA}$ average current. For machine development work, a carbon beam-stop on the straight-ahead line in the switchyard allows testing to a few hundred $\mu \mathrm{A}$ average current for short periods of time. The shielding is to be brought to full capability during the spring of 1975.

Accelerator development work concentrates on two areas: simultaneous acceleration of $\mathrm{H}^{+}$and $\mathrm{H}^{-}$beams and tuning for higher intensity. Dual bean acceleration has been demonstrated, ${ }^{1}$ but beam losses were unacceptably high. The main difficulties were in the alignment of accelerator components and in the reduction of longitudinal acceptance of the $805-\mathrm{MHz}$ linac from errors in the side-coupled structure. These problems are elaborated below. It is important to note that no exotic phenomeni which would preclude dual beam operation have been observed. For experimental physics production work up to the 20- $\mathrm{A}$ average level, it is adequate to accelerate only the $H$ beam in the linac and use various stripping stations to generate and tailor both $\mathrm{H}^{+}$and $\mathrm{H}^{-}$ beams. The present $\mathrm{H}^{-}$ion source is capable of $1-m \mathrm{~A}$ peak current, giving $60 \mu \mathrm{A}$ average, of which $33 \mu \mathrm{A}$ can be accelerated with one buncher. A prebuncher is planned, which will increase the captured current to $45 \mu \mathrm{A}$.

Exploration of the high-intensity frontier proceeds in two directions. The full $\mathrm{H}^{+}$peak current of $16 \mathrm{~mA}$ has been accelerated to $211 \mathrm{MeV}$ and 10-mA peak current to $800 \mathrm{MeV}$, in pulses $100 \mu \mathrm{s}$ long at low duty factor. Figure A-1 shows the 10-mA-peak beam-current pulse shape at $800 \mathrm{MeV}$. No evidence of beam instabilities has been seen. This mode of operation is primarily a test of the rf and field 


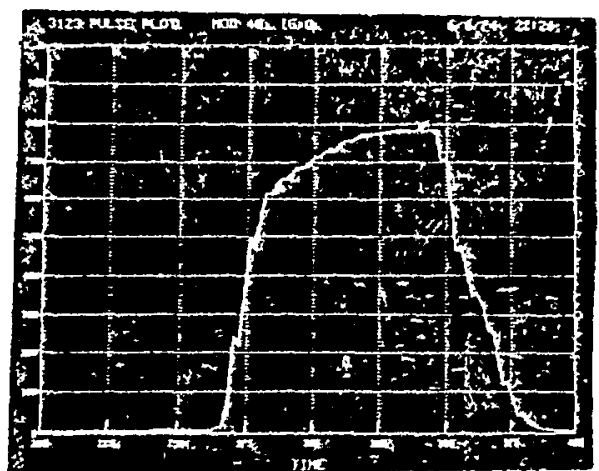

Fig. A-1. Bean-current pulse shape at $800 \mathrm{MeV}$ for a 10-mA peak current $\mathrm{H}^{+}$beam.

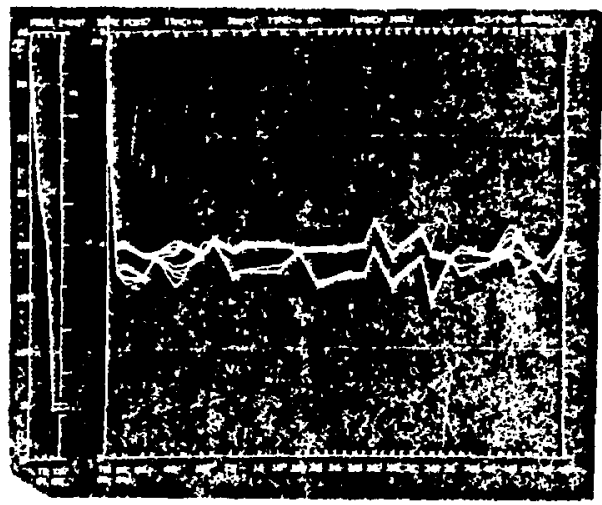

Fig. A-2. Beam-current profile along linac from injector to $800 \mathrm{MeV}$. Vertical scale is from $3 \mathrm{~mA}$ to $5 \mathrm{~mA}$. Upper trace is 4-mA peak current beam at low duty factor. Lower trace is at 5\% duty factor, giving 200- $\mu \mathrm{A}$ average current. Difference is not spil1, but only a change in ionsource output due to heating. Jagged trace is due to current-monitor systen. Spill is substantially less than $1 \%$.

control systems. At lower peak currents and higher duty factor, average currents of $200 \mu \mathrm{A}$ at $800 \mathrm{MeV}$ have been achieved for very short time pariods. Figure A-2 shows the beam-current profile along the machine. Measurements using wire scanners in the linac, the switchyard specirometer, and the EPICS channel indicate that essentially all of the beam entering the switchyard has been accelerated to the full $800 \mathrm{MeV}$. The most accurate momentum-spread measurement ${ }^{2}$ to date, made at $497 \mathrm{MeV}$, gives $\Delta p / p \leq 0.14 \%$ FWH. Figures $A-3$ and $A-4$ show beam profiles for a 2-mA-peak-current beam from 647-800 MeV and around the switchyard bend; the rotal emittance of this beam at $800 \mathrm{MeV}$ is $\sim 0.14 \pi \mathrm{mrad}-\mathrm{cm}$, and the rms emittance is $0.025 \pi \mathrm{mrad}-\mathrm{cm}$.

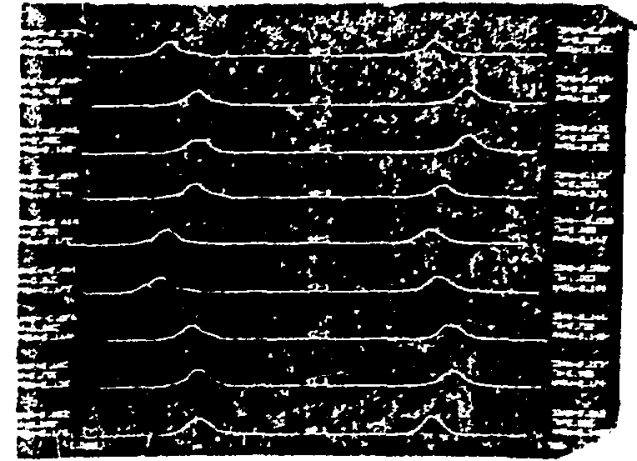

Fig. A-3. Wire-scanner beam profiles in the last 8 modules, from 647-800 $\mathrm{MeV}$, for 2-mA peak $\mathrm{H}^{+}$beam. The total emittance at $800 \mathrm{MeV}$ is $0.14 \pi \mathrm{mrad}-\mathrm{cm}$ and the rms emittance is $0.025 \pi \mathrm{mrad}-\mathrm{cm}$.

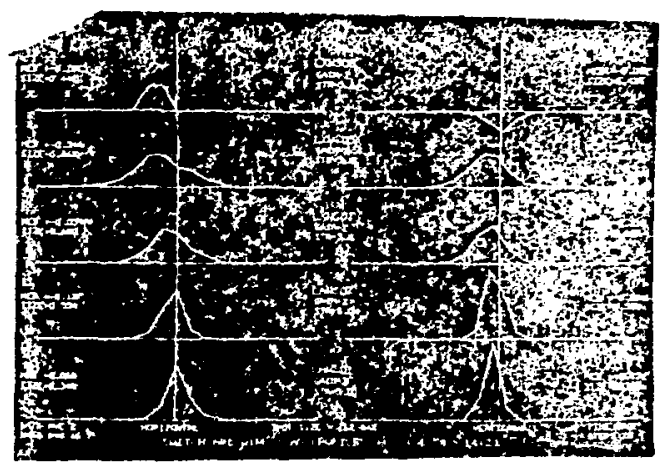

Fig A-4. $\mathrm{H}^{+}$beam profiles for a $2-\mathrm{mA}$ peak current around the switchyard bend, reading from bottom up. The dispersion is $\sim 2.26 \mathrm{~cm} / \mathrm{o}^{\circ}$ momentum at the fourth scan from the bottom. Total scanner stroke is $5 \mathrm{~cm}$.

The following paragrap̧hs outline in more detail the factors underlying these achievements and areas of present and future work.

\section{TRANSVERSE PHASE SPACE}

The alignment of the accelerator $\mathrm{rf}$ and magnetic elements is a crucial factor in the acceleration of simultaneous beams and also for high-intensity work, especially if the output energy is varied, because the required steering is a function of energy. The original alignment during construction did not meet the tolerances $( \pm 0.125-m$ random in uniform distribution on magnetic elements) due to problems with diffraction, the laser equipment used, and the dataanalysis techniques. The transition region joining 
the $201.25-\mathrm{MHz}$ and $805-\mathrm{MHz}$ 1inacs, the $805-\mathrm{MHz} 1$ inac, and parts of the switcnyard were realigned during the last year. The resulting alignment, which achieved a reproducible accuracy of $\pm 0.178 \mathrm{~mm}$ peak-to-peak, is shown in Figure A-5. Because it would have involved retuning, the accelerat or structures were not moved to a straight line; instead, the magnets were positioned on a smooth curve fitted to the tank positions. The alignment technique used a selfadjusting level for vertical measurements and a taut wire as the reference for horizontal measurements. Careful attention was paid to eliminating systematic errors in the use of the instruments. Repeated surveys were made, followed by complete data analysis, until consistency was achjeved.

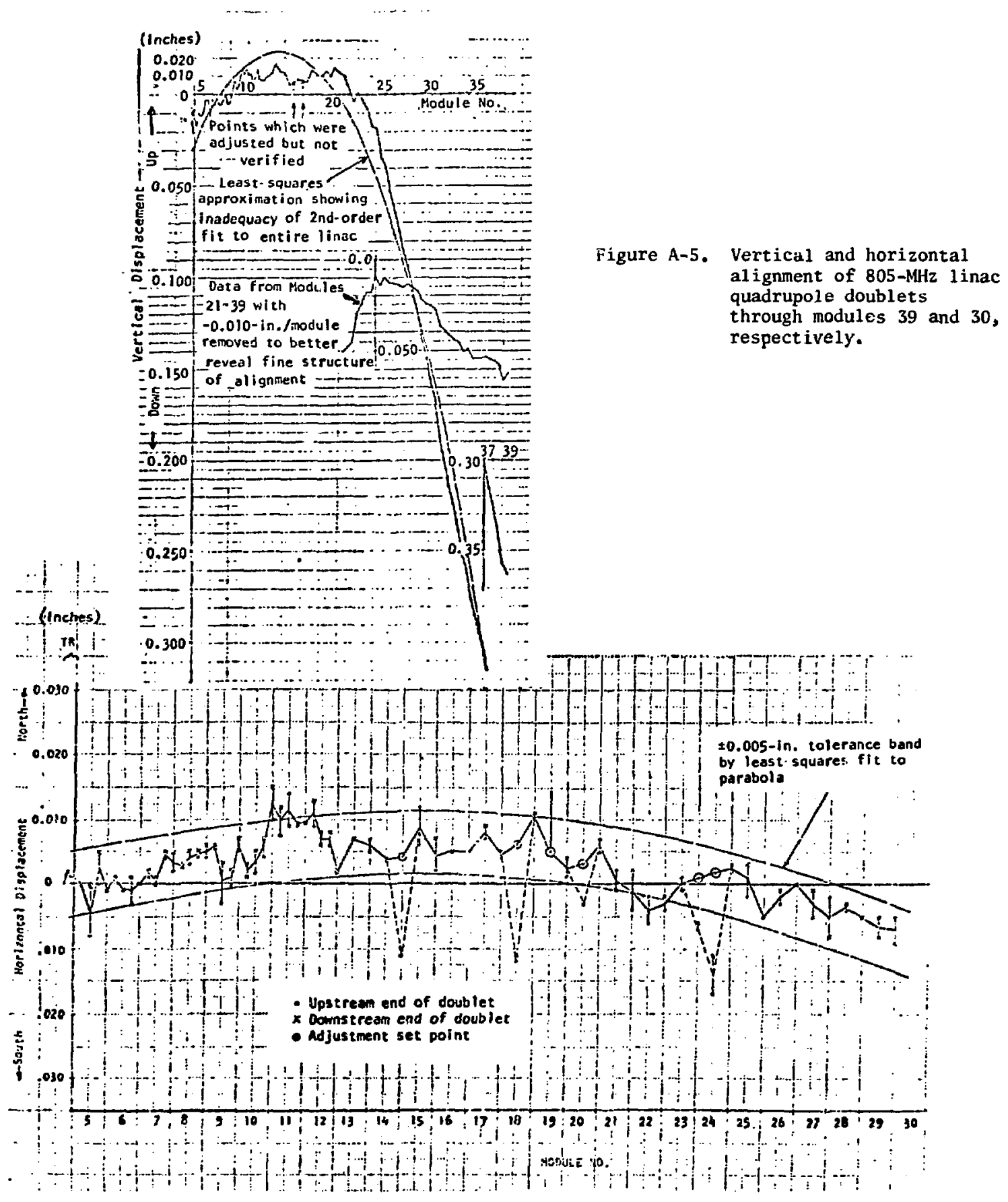


A concerted attempt was made to find settings for the $201-\mathrm{MHz}$ linac transition region which would allow operation without realignment. However, various factors indicated that such a solution, if it existed at all, would be very sensitive. It has been decided to realign the 201-MHz tanks and drift tubes and to bring the injectors, transport lines, 201-MHz linac and transition region into smooth line with the effective axis of the $805-\mathrm{MHz}$ linac. This will be done during the early months of 1975. Various alignment techniques are being explored. Three will probably be used. The first would be a completely optical alignment; the second detects the impulse imparted to a taut, current-pulsed wire if it does not lie on the magnetic axis of a quadrupole; ${ }^{3}$ and the third uses the cross generated when polarized light

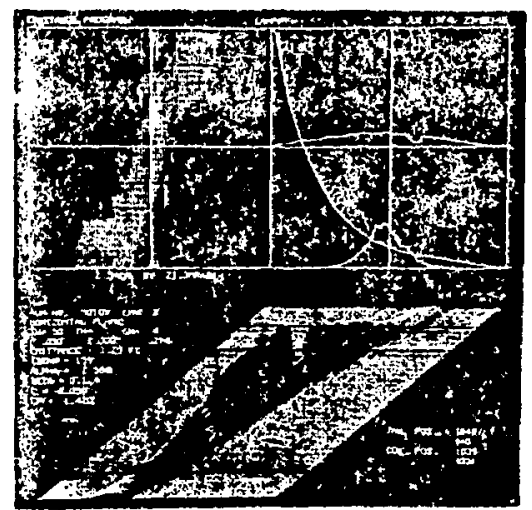

is passed through a colloidal suspension of fericsoferric particles under the influence of a quadrupole field. ${ }^{4}$ Both of the latter methods indicate the magnetic axis directly.

The emittances ( $100 \%$ of beam) at the injector column exit for $\mathrm{H}^{+}$and $\mathrm{H}^{-}$are $\sim 4 \pi$ and $5 \pi \operatorname{mrad}-\mathrm{cm}$, respectively. The beans proceed through long ( $\sim 13 \mathrm{~m})$ transport systems to the linac. Numerous criteria must be satisfied in the transport, including generally minimum size and similarity of $x$ and $y$ profiles, small size and double waists at the bunchers, size constraints at the beam chopper locations, and a match into the linac. Close agreement between theory and experiment for the $\mathrm{H}^{+}$beam is hampered by space charge and neutralization effects. Experimental emittances near the linac entrance are shown in Figure A-6 for

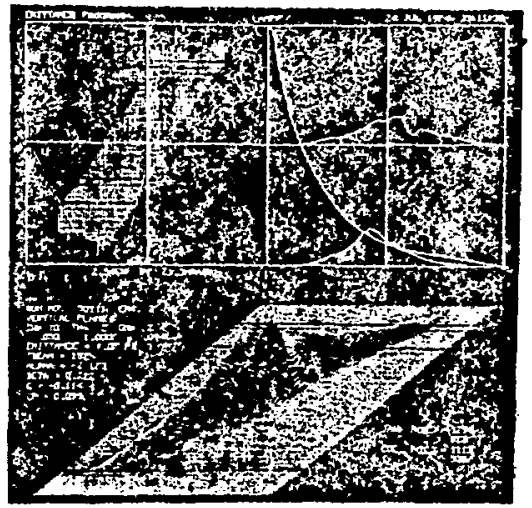

Fig. A-6. Experimental tctal emittance of $22-\mathrm{mA}$ peak $\mathrm{H}^{+}$beam near linac entrance equals $3.29 \pi \mathrm{mrad}-\mathrm{cm}$ in horizontal and $4.07 \pi$ mrad-cm in vertical.
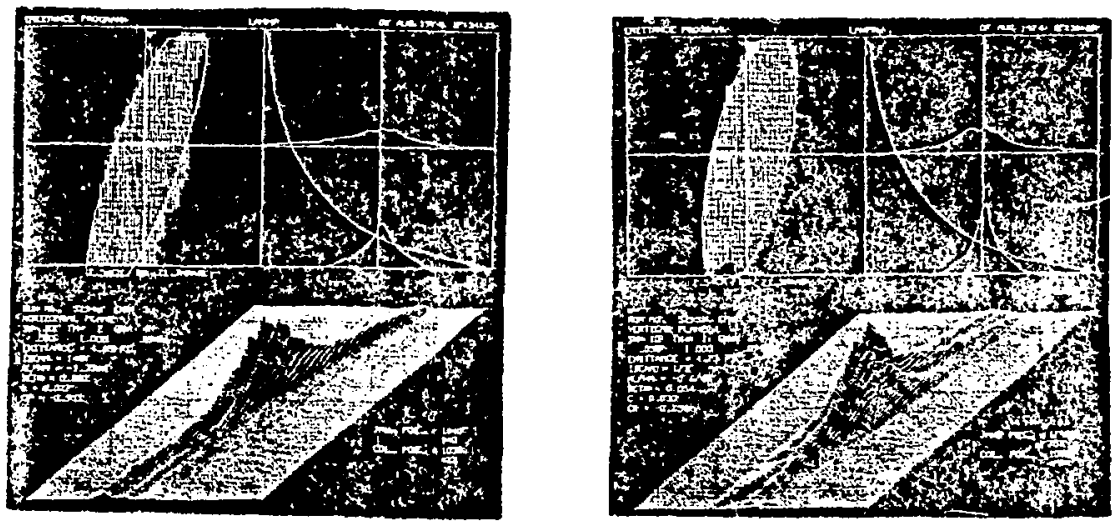

Fig. A-7. Experimental total emittance of $800-\mu A$ peak $\mathrm{H}^{-}$near 1 inac entrance equals $4.85 \pi \mathrm{mrad}-\mathrm{cm}$ in horizontal and $4.73 \pi \mathrm{mrad}-\mathrm{cm}$ in vertical. 
the $\mathrm{H}^{+}$beam. A standard 2-mA peak beam is produced for tuning purposes by scraping with a variable aperture at one upstream location. The emittance is thereby reduced to $0.7-0.8 \pi \mathrm{mrad}-\mathrm{cm}$ in both planes. The $\mathrm{H}^{-}$line is further constrained by requiring achromatic bends to minimize dispersion of the throe primary varieties of $\mathrm{H}^{-}$ions derived from different reactions, and the requirement for matching to the final set of quads which are set for the $\mathrm{H}^{+}$beam. Figure $\mathrm{A}-7$ shows $\mathrm{H}^{-}$emittances at the same location. The beams then proceed through the $201.25-\mathrm{MHz}$ linac and the transition region. The number and 10cation of steering and position-sensing devices in the $201.25 \mathrm{MHz}$ linac is insufficient for correction of simultaneous beams, hence the need for realignment.

Wire scanners are used in the $805-\mathrm{MHz}$ linac to obtain transverse data. Emittance can be calculated from a series of wire scanner profiles. For peak $\mathrm{H}^{+}$ and $\mathrm{H}^{-}$currents of $2 \mathrm{~mA}$ and $400 \mu \mathrm{A}$, respectively, the rms emittance at $100 \mathrm{MeV}$ is $\sim 0.05-0.06 \pi \mathrm{mrad}-\mathrm{cm}$, damping at $800 \mathrm{MeV}$ to $\sim 0.015-0.02 \pi$, consistent with the transverse $B Y$ damping factor of 3.3. The present switchyard optics require accelerator emittances at least this good for low-loss transport. Studies are now in progress to apply systematic scraping at various locations to remove halo and tail components. This tailoring of the phase space prior to $100 \mathrm{MeV}$ will substantially aid the achievement of reproducible high-intensity beams. The absence of fast, noninterfering position and profile monitoring equipment along the accelerator is a significant hindrance to tuning procedures. The effective steering of simultaneous beams in the strong-focusing $805-\mathrm{MHz}$ linac requires the proper symmetries in the steering dipoles. 5

III. LONGITUDINAL PHASE SPACE

The $\mathrm{H}^{-}$beam consists of two main components differing in energy by $\sim 3 \mathrm{keV}$, and a smaller third component separated by $\sim 10 \mathrm{keV}$ which may be scraped off in the transport system. The $\mathrm{H}^{+}$beam is about 65\% protons at full design current of $36-\mathrm{mA}$ peak with the present aperture. The injectors are operated at fixed settings as much as possible, with peak current variation achjeved by scraping in the transport system. Intensive work over the past year has resulted in much improved long-term stability of the energy of both injectors and in the mirimization of the number of and time to recover from arciowns,

The phase and amplitude of the $r f$ fields in the $201.25-\mathrm{MHz}$ linac are adjusted by varying the phase or amplitude of the $\mathrm{rf}$ and observing the amount of current accelerated to the proper energy, as determined by an absorber and collector at the end of each module. The curves so generated are compared to corresponding analytical curves derived from computer models, and anplitude or phase is adjusted until satisfactory agreement is achieved. The method, while not absolute, contains many correlations and yields consistent results. Tuning with the $\mathrm{H}^{+}$beam is the most precise, since it contains only one energy component.

The longitudinal dynamics and associated instrumentation in the $805-\mathrm{MHz}$ linac have received major attention in the last year. Onerational awkwardness and calibration problems make the switchyard spectrometer useful only as a secondary check during tuneup. The primary tuneup of phase and amplitude is now accomplished with a time-of-flight $(\Delta t)$ method. Loops are placed between modules, and the phase of beaminduced signals is measured. With the module acce1erating, the amplitude is set to produce agreement with the major features of an analytically derived energy gain vs phase curve such as that shown in Figure A-8. The phase is then set more precisely by measuring with the beam alternately accelerated and coasted through the module. The apparatus is completely electronic, fast, and yery precise. It is also being used to monitor the machine during production use to aid in eliminating drifts and other shifts in the beam characteristics.

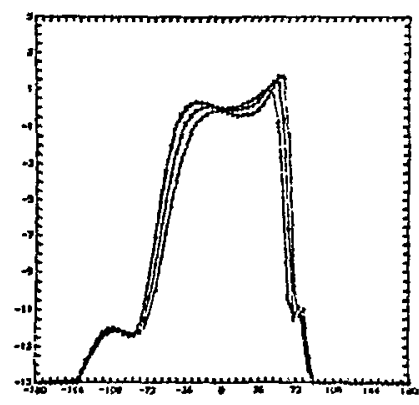

Fig. A-8. Analytical energy-gain vs rf-phase curve for module 8, which accelerates from -139 $\mathrm{MeV}$ to $\sim 153 \mathrm{MeV}$, shown in $\mathrm{MeV}$ and degrees as deviation from design value. 
Analysis of discrepancies observed in the $\Delta t$ measurements has led to the discovery of the probable major cause of longitudinal beam loss in the $805-\mathrm{MHz}$ linac. The acceptance of the design linac is shown in Figure A-9. Length tolerance buildup during the manufacturing of the accelerator and length errors introduced by faulty tuning procedures in the lowenergy modules resulted in severe distortions in the longitudinal acceptance and a general reduction in area by a factor of two, as shown in Figure A-10.
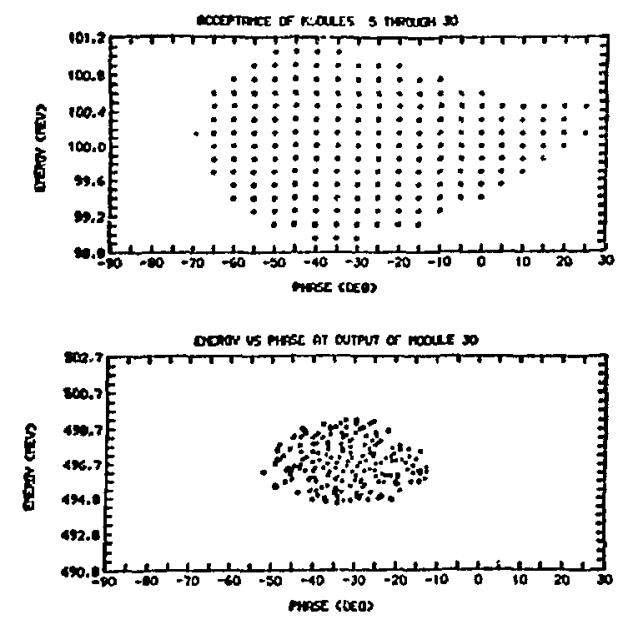

Fig. A-9. Original design linac.
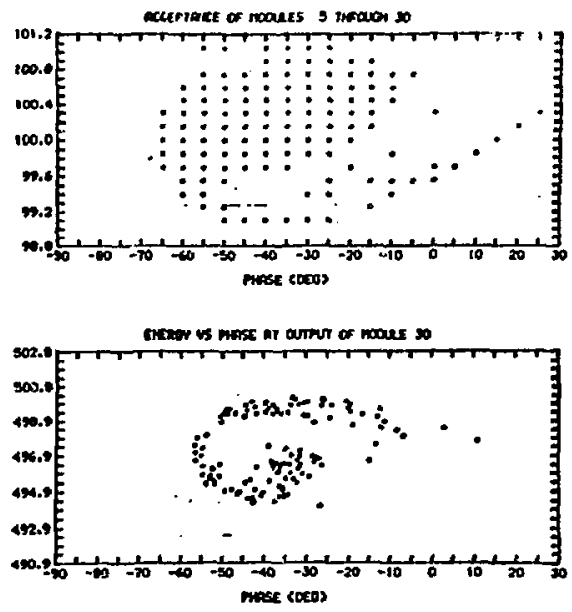

Fig. A-10. Including measured errors in physical lengths, but with original design $r f$ amplitudes and phases.
If the structure errors had been absorbed by the intramodule drifts so that if the module length were exact, there would be no problem; however, the drift spaces also have errors. As an indication of the sensitivity of the effect, a length tolerance of about $\pm 0.3 \mathrm{~mm}$ on the module length $(\sim 20 \mathrm{~m})$ is required to maintain the $\pm 1^{\circ}$ rf phase tolerance that is expected between tanks and between modules. A procedure has been devised ${ }^{6}$ to partially alleviate the problem. First, each module is analytically
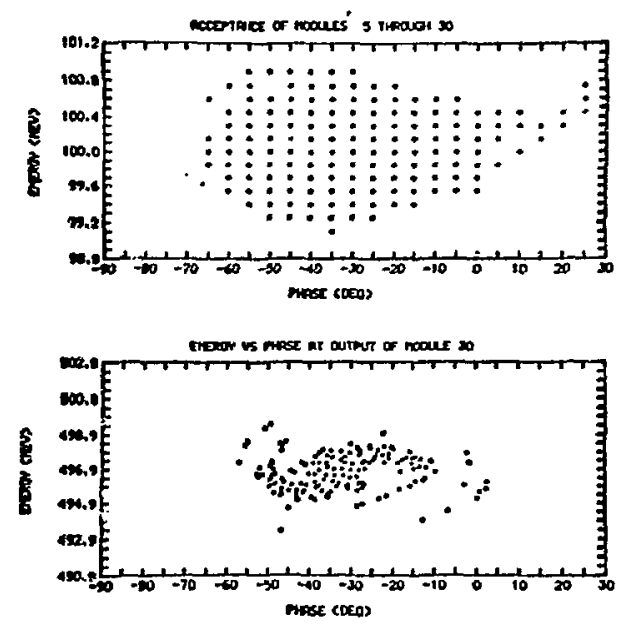

Fig. A-11. Compromise linac design, with original design rf amplitudes.
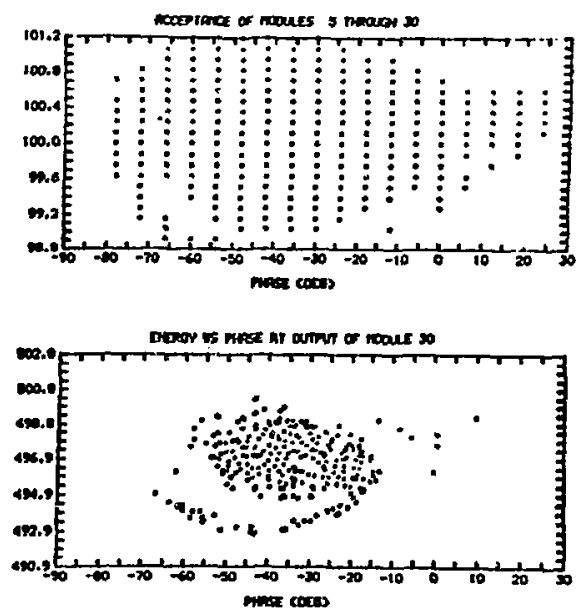

Fig. A-12, Compromise linac design with rf amplitudes raised $5 \%$, and synchronous phase shifted to $-35^{\circ}$. 
"redesigned" using its measured lengths to find an input energy and phase which minimize the errors within that module. Then a fit is made along the entire linac to achieve the best compromise for moduleto-module tuning. The resulting admittance is shown in Figure A-11. A general increase in the design field level of $5 \%$ can then increase the area as shown in Figure A-12. Subsequent tuneup of the accelerator using these new settings was substantially more successful in eliminating spill and in lower sensitivity to parameter shifts. Studies are now in progress to determine the best way to physically correct the most important length errors during the 1975 shutdown. Other studies of the $\Delta t$ method have led to increased understanding of the effects of various errors and the way that errors can propagate along the machine.

\section{MAINTENANCE AND OPERATIONS}

The development of consistent operating and maintenance procedures is vital if consistent and stable operation at higher intensities is to be achieved with minimum downtime, and work has begun in these areas. Computer-based tools will be used to measure machine performance over long time intervals.

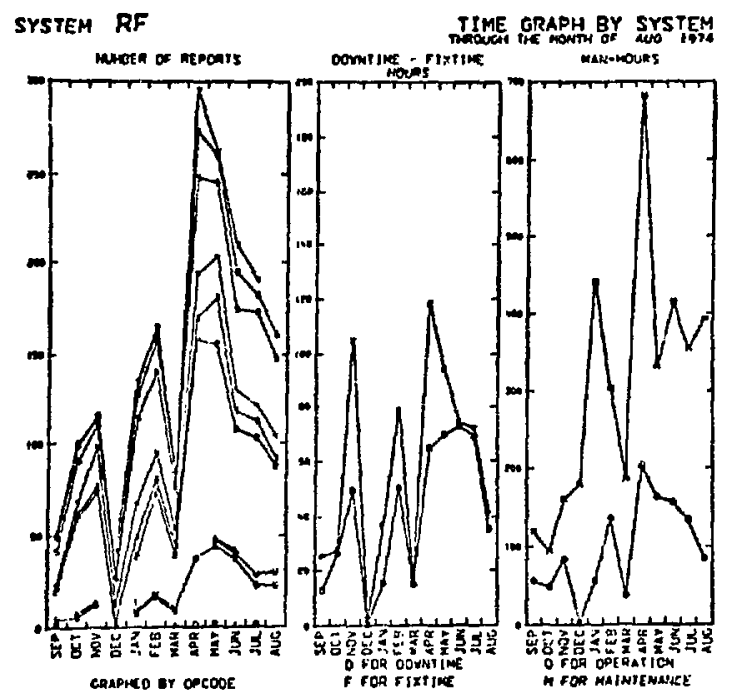

The central control system can monitor many signals and show deviations from standard settings, correlations between signals and so on. This capability has only begun to be exploited, but will serve as a primary tool in finding the source oi drift and other long-term effects.

An inventory has been made of all machine equipmenc, and systems reviewed for proper documentation, spares and stock inventory, needed improvements, and other support factors. The information accumulated is stored in a computerized data-base, from which it can be retrieved in various formats. For instance, the data-base can be asked which items in the $r f$ system are still deficient in spare parts, or schedules for preventative maintenance can be generated each week. A daily reporting system has been initiated in which equipment malfunctions are reported on small cards. Each morning, a list is made, with the aid of the computer data base, and sent to the support personne1. The information is also stored in the data base, and equipment operating history can be studied over a period of time. Figure A-13 shows a composite graphical history of the $r f$ and vacuum systems over the past year. Planned extensions to these programs include various reliability and availability estimates.

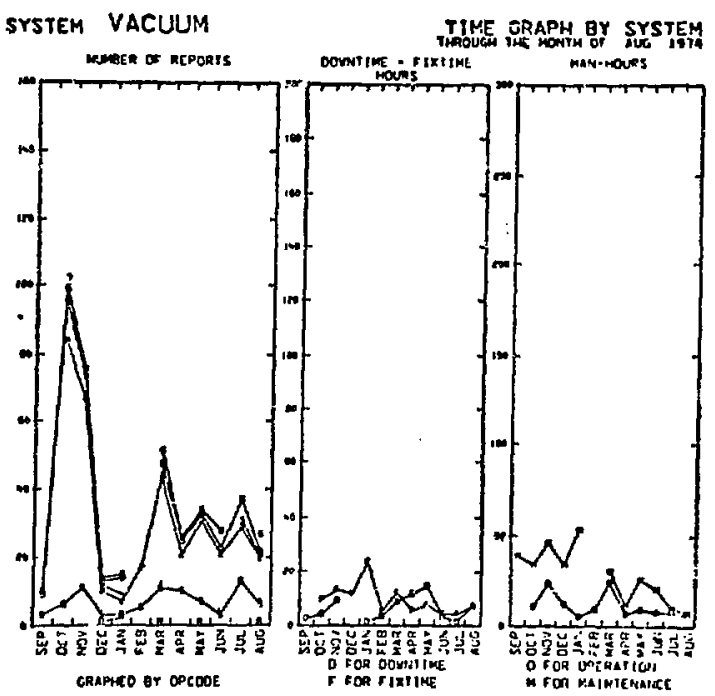

Fig. A-13. RF and vacuum system histories from August 1973 through August 1974, showing,the total number of trouble reports filed, and downtime and man hours associated with the repair: The initial upward trend for $\mathrm{rf}$ reflects the starting of the reporting system as more and more equipment was included. Vacuum work was high again in September 1974, reflecting maintenance on pumps nearing the end of pumping element lifetimes. 


\section{References}

1. Quarterly Report on the Medium-Energy Physics Program for Period Ending April 30, 1973, Los Alamos Scientific Laboratory report LA-5358-PR.

2. M, $\mathrm{D}$. Cooper, "Time-of-Flight Measurements at the LAMPF Low-Energy Pion Channel," Los Alamos Scientific Laboratory report LA-5529-MS (March 1974).

3. R. M. Main, "Alignment of Drift Tube Quadrupole Lenses Using a Pulsed Wire," Proceedings of the IV Internationa1 Conference on Magnet Teuhnology, CONF-720908, Brookhaven, 1972.

4. J. K. Cobb and J. J. Muray, "Magnetic Field Measurement and Spectroscopy in Multipole Fields," IEEE Trans. Nucl. Sci., Vol. NS-12, No. 3 (June 1965).

5. K. R. Crandall and W. E. Jule, "Simultaneous Steering of $\mathrm{H}^{+}$and $\mathrm{H}^{-}$Beams at LAMPF," Proc. IXth Intern. Conf. on High Energy Accelerators, SLAC (May 2-7, 1974).

6. K. R. Crandall, " $803-\mathrm{MHz}$ Linac Design Modifications Due to Length Errors," Los Alamos Scientific Laboratory, unpublished data, 1974. 


\section{APPENDIX B}

\section{LAMPF - RELATED PUBLICAT IONS}

k. Crandall, "Computation of Electrostatic and Rapidiy Pulsed Magnetic Fields," Los Alamos Scientific Laboratory report LA-3416.

L. Rosen, "Outline of the Proposal for a Meson Facility at Los Alamos, "Los Alamos Scientific Laboratory report LA-2935-MS.

C. Critchfield, L. Heller, M. Lee, and J. Young, "Pions, Muons, and Nuclear Structure," Los Alamos Scientific Laboratory report LA-3009-MS.

L. Rosen and D. Nagle, "Introduction to the Proposed Los Alanos Meson Project," Los Alamos S:ientific Laboratory report LA-3025-MS.

L. Rosen, "Nuclear Physics Applications of a HighFlux Meson Facility," Los Alamos Scientific Laboratory report LA-3030-MS.

H. 6. Worstell, "Local Cooling System for 800-MC Cloverleaf Prototype," Los Alamos Scientific Laboratory internal report $\mathrm{P}-11 / \mathrm{HGW}-2$.

B. C. Knapp, "Chain of Coupled Oscillators of Two Kinds," Los Alamos Scientific Laboratory internal report $P-11 / B C K-1$.

D. Nagle, B. Knapp, and E. Knapp, "Perturbation Theory for the Doubly Periodic Gain," Los Alamos Scientific Laboratory internal report $P-11 / D N / B K / E K-1$.

G. R. Swain, "Free Field Computer Card Read Routine," Los Alamos Scientific Laboratory internal report MP-3/GRS/ 1

G. R. Swain, "Notes on Tuning a Chain of Doubly Coupled Resonators," Los Alamos Scientific Laboratory internal report MP-3/GRS/2.

N. A. Lindsay and R. A. Jameson, "LAMPF Phase and Amplitude Control System," Los Alamos Scientific Laboratory internal report MP-2/NAL/RAJ-1.

H. G. Worstel1, "Safe Operation of Ion Pumps," Los Alamos Scientific laboratory internal report $\mathrm{MP}-3 / \mathrm{HGW}-4$.

J. R. Ruhe, "Investigation into Possible Mechanical Redesign of the RCA Coaxitron Anode." Los Alamos Scientific Laboratory internal report MP-3/JRR-1.

M. Rich and W. M. Visscher, "A Green's Function Appreach to the Calculation of Drift Tube Cavities," Min. Conf. on Proton Linear Accel., July 20-24, 1964 (Midwestern Universities Research Association).

H. C. Hoyt, "Drift Tube Calculations," Ibid.

M. Rich, "Beami Dynamics Calculations for AlvarezType Linear Accelerators," Ibid.

D. E. Nagle, "Coupled Resonator Model of Linear Accelerator Tanks," Ibid.

E. A. Knapp, "800 MeV RF Structures," Ibid.
D, E. Nagle, "The LASL Meson Factory," Ibid.

M. Jakobsor, and W. M. Visscher, "Numerical Study of Particle Dynamics in a High-Energy Proton Linac," Ibid.

R. A. Jameson, "RF Phase and Amplitude Control," Ibid.

H. C. Hoyt, "Numerical Studies of the Shapes of Drift Tubes and Linac Cavities," First Nat'1. Part. Accel. Conf., Mar. 10-12, 1965 (Washington, DC).

M. Rich, "Beam Dynamical Calculations with Realistic $F^{*}$ elds in a Drift Tube Linear Accelerator," Ibid.

W. M. Visscher, "Optimization of Magnetic Lenses for Waveguide Portion of a Proton Linac," Itid.

R. W. Freyman, "A 40-Kilovolt, 125-Ampere Hard Tube Modulator for Accelerator Service," Ibid.

T. M. Putnam, R. A. Jameson, and T. M. Shul theis, "Application of a Digital Computer to the Control and Monitoring of a Proton Linac," Ibid.

E. A. Knapp, "Design, Construction, and Testing of RF Structures for a Proton Linear Accelerator," Ibid.

R. A. Jameson, T. F. Turner, and N. A. Lindsay, "Design of the RF Phase and Amplitude Control Sy stem for a Proton Linac," Ibid.

J. E. Brolley, Jr., C. R. Emigh, and D. W. Mueller, "Electron Analog Tests of Proton Linac Structures," Ibid.

J. R. Parker, J. D. Doss, R. W. Freyman, E. A. Knapp, and W. J. Shlaer. "High-Power Tests on a Cloverleaf Carity," Ibid.

B. C. Knapp, E. A. Knapp, G. J. Lucas, and J. M. Potter, "Resonantly Coupled Accelerating Structures for High-Current Linacs," Ibid.

R. L. Gluckstern and H. S. Butler, "Transverse Beam Blow-Up in a Standing Wave Linac Cavity," Ibid.

B. C. Knapp, E. A. Knapp, G. J. Lucas, D. E. Nagle, and J. M. Potter, "Electrical Behavior of Long Linac Tanks and a New Tank-Coupling Scheme," Ibid.

R. A. Jameson, "Transient Response of a Chain of Coupled Circuits," Los Alamos Scientific Laboratory internal report $\mathrm{P}-11 / \mathrm{RAJ}-3$.

H. G. Worste11, "800-Mc Cloverleaf Evacuation System," Los Alamos Scientific Laboratory internal report $\mathrm{P}-11 / \mathrm{HGW}-3$.

B. C. Knapp, "Steady State and Transient Behavior of a Chain of Coupled Resonant Circuits," Los Alamos Scientific Laboratory internal report $\mathrm{P}-11 / \mathrm{BCK}-2$.

J. R. Ruhe, "Copper-clad Steel Welding Technique Developments," Los Alamos Scientific Laboratory internal report $M P-3 / J R R-2$.

M. Jakobson, "Notes on Scattering and Transport of the Primary Proton Beam," Los Alamos Scientific Laboratory internal report MP/MJ-5. 
S. Rislove, "200 MHz (Sector A) Cooling Water Criteria," Los Alamos Scientific Laboratory internal report $\mathrm{MP}-3 / \mathrm{SR}-1$.

M. Jakobson, "Beam Divergence Introduced by Magnetic and Electric Deflectors," Los Alamos Scientific Laboratory internal report MP/MJ- 6 .

N. A. Lindsay, R. A. Jameson, and J. H. Richardson, "LAMPF RF Phase and Amplitude Control System," Los Alamos Scientific Laboratory internal report MP $-2 / \mathrm{NAL} / \mathrm{RAJ} / \mathrm{JR}-\mathrm{I}$.

E. D. Bush and D. R. Copenhagen, "Grouping Criteria for 201.25 $\mathrm{MHz}$ Linac Drift Tube Quadrupoles," Los Alamos Scientific Laboratory internal report MP-3/EDB/DRC-1.

R. A. Jameson and N. A. Lindsay, "Notes on Phase and Amplitude Permissive System," Los Alamos Scientific Laboratory internal report MP-2/NAL/RAJ-2.

C. R. Enigh, "Double Dri r Buncher--Part A: Without Space Charge," Los Alamos Scientific Laboratory internal report $M P-4 / C R E-1$.

C. R. Swain, "Circuit Analog Techniques for Analys is of Resonant ly-Coupled Linear Accel erator Structures," Los Alamos Scientific Laboratory internal report MP-3/GRS- 3 .

H, G. Worstel1, "Design, Development, and Fabrication of Multicavity $805 \mathrm{MHz}$ Accelerator Tanks," Los Alamos Scientific Laboratory internal report MP - 3/ HGW-5.

K. R. Cranda11, "A Numerical Experiment on SpaceCharge Effocts," Los Alamos Scientific Laboratory internal report $\mathrm{ill}-4 / \mathrm{KC}-1$.

D. R. Copenhagen, E. A. Knapp, and J. M. Potter, "Operating Characteristics of a Full Power Cloverleaf Accelerator Tank," Los Alamos Scientific Laboratory internal report MP-3/DRC-1.

J. R. Parker, "LAMPF Cable Plant Wiring," Los Alamos Scientific Laboratory internal report MP-1-1506.

D. A. Swenson, "Optimum Geometries and Field Strengths for Alvarez Linacs," Los Alamos Scientific Laboratory report iA-3567.

K. R. Crandal1, "Computation of Charge Distribution On or Near Equipotential Surfaces," Los Alamos Scientific Laboratory report LA-3512.

"Proceedings of the 1966 Linear Accelerator Conference," Oct. 3-7, 1966 (Los Alamos, NM), Los Alamos Scientific Laboratory report LA-3609.

H. S. Butler, "Computer Control of the Les Alamos Linear Accelerator," U.S. Nat'1. Part. Accel. Conf., Mar. 1-3, 1967 (Washington, DC).

E. C. Budge, "Data Transmission Across High Voltage Interfaces via Light Links," Ibid.

D. E. Nagle, "Advances in the Design of Proton Linear Accelerators--Meson Factories and Injectors for Synchrotons," Ibid.
R. A. Jameson and W. J. Hoffert, "Fast Automatic Phase and Amplitude Control of High-Power RF Systems," Ibid.

D. C. Hagerman, "High Duty Factor RF Sources at 800 $\mathrm{MHz}, "$ Ibid.

?. P. Severns, "Crossing High-Voltage Interfaces with Bandwidth Signals," Ibid.

T. J. Boyd, Jr. and R. A. Jameson, "Optimun Generator Characteristics of RF Amplifiers for Heavily Beam-Loaded Accelerators," Ibid.

D. J. Liska, "Computer vesign of LHF Power Amplifier Tubes," Ibid.

J. D. Doss, "Pulse Gate," Electronics.

L. Rosen, "Meson Factories," Physics Today, Dec. 1967.

L. Rosen, "Why Meson Factories?" Physics Teachers.

F. P. Agee and L. Rosen, "Calculations of Neutron Cross Sections Using a Local Optical Potential with Average Parameters," Nuclear Data, Dec. 1966, Wor IdWide News of Comp, in Nuclear Physics.

M. Bolsterli, F. Low, L. Rodberg and J. Young, "Estimates of Maximum Energy $\pi$ Production on Nuclei, $K \Lambda$ Production on Nuclei, and Strangeness-Violating $\Lambda$ Production on Neutrons by $800 \mathrm{MeV}$ Protons," Los Alamos Scientific Laboratory internal report MP-DO/FEL-1.

C. R. Emigh, "The 'Pierce'-Geometry, An Accelerating Column Design," Los Alamos Scientific Laboratory internal report $M P-4 / C R E-2$.

V. W. Hughes, "Muonium," Los Alamos Scientific Laboratory internal report $M P-D O / V W H-1$.

v. W. Hughes, "Tests of Quantum Electrodynamics and Properties of the Muon," Los Alamos Scientific Laboratory internal report MP-DO/VWH- 2 .

V. W. Hughes, "Muonium Chemistry; Muon Capture," Los Alamos Scientific Laboratory internal report MP-DO/VWH-3.

D. A. Swenson, "Bucket Rotator," Los Alamos Scientific Laboratory internal report MP-4/DAS-2.

G. R. Swain, "Degradation of Long Tank Performance with Tuning Errors--Summary of Calculated Results," Los Alamos Scientific Laboratory interval report MP-3/GRS-5.

R. R. Stevens, Jr., "Survey of Polarized Ion Sources Suitable for LAMPF," Los Alamos Scientific Laboratory internal report MP-4/RRS- 1 .

T. F. Turner, "Pulse Current Transformers," Los Alamos Scientific Laboratory internal report MP-2/TFT-7.

D. J. Liska, "Notes on Single Particle Theory in Crossed-Field Devices," Los Alamos Scientific Laboratory internal report $\mathrm{MP}-2 / \mathrm{DJL}-1$. 
E. D. Bush, Jr. "Mechanical Design of Drift Tubes and Quadrupole Magnets for the Alvarez Linac," Los Alamos Scieitific Laboratory internal report MP-3/EDB-1.

E. A. Knapp, P. W. Allison, C. R. Emigh, L. N. Engel, J. M. Potter, and W. J. Shlaer, "Accelerating Structure Research at Los Alamos," Los Alamos Scientific Laboratory internal report MP-3/EK/PA/CE/LE/ JP/WS-1.

J. M. Potter, "Transverse Modes in a Resonantly Coupled Accelerator," Los Alamos Scientific Laboratory interial report $M P-3 / J M P-2$.

L. N. Enge i and E. A. Knapp, "Tuning and Field Measurement Techniques in a Resonantly Coupled Accelerator Tank," Los Alamos Scientific Laboratory internal report MP-3/LNE/EAK-1.

H. G. Worstel1, "LAMPF 201 MHz Vacuum System," Los Alamos Scientific Laboratory interna? report $\mathrm{MP}-3 / \mathrm{HGW}-6$.

D. E. Nagle, "Linear Accelerators for" Protons--New Developments," Science, Feb. 23, 196".

N. Metropolis, J, R. Pasta, M. Fraser, "Simulation of the SEL-810A Computer on MANIAC II (SELMA)," Los Alamos Scientific Laboratory report LA-3640, Feb. $27,1967$.

E. R. Flynn and L. Rosen, "Interactions of 22-MeV $\mathrm{He}^{3}$ Particles with $\mathrm{Fe}^{56,58}$ and $\mathrm{Ni}^{58}$," Phys. Rev. 153, 1228 (1967).

"Ihe Proposed Los Alamos Meson Physics Facility and Some Possible Applications to Biomedicine," Progress in Biomedical Eng., 1967.

C. R. Emigh and A. C. Hindmarsh, "Double Drift Buncher," Los Alamos Scientific Laboratory internal report MP-4/CRE, ACH- 1 .

R. R. Stevens, Jr., "Beam Steering Using Unbalanced Excitation of Quadrupole Magnets," Los Alamos Scientific Laboratory internal report MP-4/RRS-2.

D. A. Swenson, "Stem Perturbations in Alvarez Linacs," Los Alamos Scientific Laboratory internal report MP-4/DAS-3.

K. R. Crandal l, "MRA, A FORTRAN IV Coce to Simulate Beam Bunching," Los Alamos Scientific Laboratory internal report $\mathrm{MP}-4 / \mathrm{KC}-3$.

K. R. Cranda11, "Optimum Buncher Parameters for Small Beam Currents," Los Alamos Scientific Laboratory internal report $\mathrm{MP}-4 / \mathrm{KC}-2$.

G. R. Swain, "FARM - A Computer Code for Accelerator Radial Motion Studies," Los Alamos Scientific Laboratory internal report MP-3/GRS-6.

D. A. Swenson, "Some Features of the Resonantly Coupled Alvarez Linac," Lo. Alamos Scientific Laboratory internal report MP-4/DAS- 4 .
H. Vogel and S. Vegors, "Magnetic Field Measurements on the Exciters for the Argonne Pion Focusing Horn," Symposium on Srace Mag. Exp. E Tech., Aug. 28-30, 1967.

D. A. Swenson, E. A. Knapp, J. M. Potter, and E. J. Schneider, "Stabilization of the Drift Tube Linac by Operation in the $\pi / 2$ Cavity Mode," 6th Internat'l. Conf. on Hi-Energy Accelerators, Sept. 11-15, 1967; Los Alamos Scientific Laboratory internal report $\mathrm{MP}-3-3$.

E. A. Knapp, J. M. Potter, W. J. Shlaer, and G. R. Swain, "Resonant Side-coupled Cavity Electron Accelerators," Ibid., Los Alamos Scientific Laboratory internal report $\mathrm{MP}-3-4$.

L. Rosen, "Prospects and Uses for Meson Factories," Ibid.

J. M. Potter, "An Elementary Perturbation Analys is Applicable to Resonant Coupled Accelerating Structures," Los Alamos Scientific Laboratory internal report MP-3/JMP-3.

E. A. Knapp, "LAMPF Design Status," AWU/U of Wyo/ LAMPF Meeting, 1968.

D. R. F. Cochran, "Experimental Area Design," Ibid.

L. Rosen, "The Political, Financial, and Physical Status of LAMPF," Ibid.

E. A. Knapp, "On a Method of Tuning the Resonant Couplers in a Drift Tube Accelerator," Los Alamos Scientific Laboratory internal report MP-3/EAK-1.

D. A. Swenson and A. Benton, "Calculation of BNL Drift Tube Table," Los Alamos Scientific Laboratory interna' report MP-4/DAS/AB(BNL).

D. A. Swenson, "Interpretation of Field Distributions in Variable Cell Length Model," Los Alamos Scientific Laboratory internal report MP-4/DAS-5.

D. R. F. Cochran and M. Jakobson, "Radiation Through Beam Channels in the Target Cell Shielding," Los Alamos Scientific Laboratory internal report MP-6/DRFC/MJJ-1.

H. Butler and M. Jakobson, "Note on Pion Transport Channels," Los Alamos Scientific Laboratory internal report $M P / H B M J-1$.

H. G. Worstel1, "Preliminary Parameters of 201.25 Tanks to Determine Clad Plate Thickness and Sizes," Los Alamos Scientific Laboratory internal report MP-3-2.

G. R. Swain, "Analysis of Multiply-Coupled Resonators Using a Matrix Formulation," Los Alamos Scientific Laboratory internal report MP-3/GRS-7.

K. R. Crandall, "Double Drift Buncher, Part. C: A Comparison with the Simple Buncher and the Harmonic Buncher," Los Alamos Scientific Laboratory internal report $\mathrm{MP}-4 / \mathrm{KRC}, \mathrm{CRE}-1$.

H. S. Butler, "The Impact of Computers on the Control of Particle Accelerators," IEEE Spring Mtg. Mar. 1968. 
H. S. Butler, R. A. Gore, and D. T. Van Buren, "Computer Control of a Linear Accelerator," IFAC-IFIP Symposium, June 17, 1968.

D. C. Hagerman and R. A. Jameson, "The Los Alamos Meson Physics Facility Accelerator," Symp. on Microwave Power, Mar. 21-23, 1968.

G. R. Swain, "Sensitivity to Tuning Errors for Multiply-Coupled Biperiodic Chain of Resonators Terminated in Full Cells," Los Alamos Scientific Laboratory internal report $\mathrm{MP}-3-8$.

D. R. Machen, R. A. Gore, and D. W. Weber, "A Compact Data Acquisition and Control Terminal for Particle Accelerators," 1969 Part. Accel. Conf.

A. Harvey and S. A. Walker, "Mineral-Insulated Magnets for High-Radiation Environments," IEEE Trans. NS (1969 Corif.)

J. R. Parker, "Radiation Safety Irstrumentation for a High-Intensity, High-Duty Accelerator," Heal th Phys. Soc. (1970).

K. R. Cranda11, "Beam Basis for Estimating Tolerance Requirements for the Side-Coupled Linac," Los Alamos Scientific Laboratory internal report $M P-4 / K C-4$.

E. D. Bush, J. R. Ruhe, E. J. Schneider, and P. J. Stroik, "Two Cell 201.25 MHz DRIFT Tube Linac Power Model," Los Alamos Scientific Laboratory internal report $\mathrm{MP}-3 / \mathrm{EDB} / J \mathrm{RR} / \mathrm{EJS} / \mathrm{PJS}-1$.

P. W. Allison, "Emittance Scanner," Los Alamos Scientific laboratory internal report MP-4/PA- 2 .

P. W. Allison, "Transport System Optics," Los Alamos Scientific Laboratory internal report MP-4/PWA-1.

J. E. Stovall, "Comparison of Two Methods for Calculating Space Charge Effects in Drift Tube Linacs," Los Alamos Scientific Laboratory internal report MP-3-10.

R. R. Silbar, "Beam Transport from Last Target to Beam Stop," Los Alamos Scientific Laboratory internal report MP-6 RRS/1.

P. W. Allison and C. R. Emigh, "On the Feasibility of $\mathrm{H}^{-}$Ion Acceleration by LAMPF," Los Alamos Scientific Laboratory internal report MP-4/PWA,CRE-1.

J. E. Stoval1, "Selection of Quadrupole Strengths for Drift Tube Linac," Los Alamos Scientific Laboratory internal report MP-3-38.

E. D. Bush, "201.25 MHz Drift Tube Linac Quadrupole Dagnet Field Surveys," Los Alamos Scientific Laboratory internal report $\mathrm{H}$ P-3-39.

E. A. Knapp, "Side-Coupled Cavity-Coupling Parameters," Los Alamos Scientific Laboratory internal report MP-3-340.

L. A. Rosen, "Possibilities and Advantages of Using Negative Pions in Radiotherapy," 14th Mtg. of Amer. Nucl. Soc., June 1968.
C. R. Emigh, "The Double Drift Buncher," 1968 Linac Conf., May 1968.

C. R. Enigh and D. W. Mueller, "The LASL High-DutyFactor Ion Source, Ibid.

P. W. Allison and R. R. Stevens, Jr., "The Beam Transport Design for the LAMPF Injector," Ibid.

D. E. Nagle, "Linear Accelerator for Unstable Particles," I bid.

G. G. Ohlsen, J. L. McKibben, R. R. Stevens, Jr., and G. P. Lawrence, "Depolarization and Emittance Degradation Effects Associated w/Charge Transfer in a Magnetic Field," Nuc. Instr. \& Methods, 1969.

L. Rosen, "Pions and Muons in Medicine," 12th Mtg. of Amer. Assoc. of Physicists in Med., 1970.

E. A. Knapp and w. J. Shlaer, "Design and Initial Performance of a $20-\mathrm{MeV}$ High-Current Side-Coupled Cavity Electron Accelerator," 1968 Linac Conf., May 1968.

G. R. Swain, R. A. Gore, and R. A. Jameson, "Temperature Control for Maintaining Resonance of Linac Tanks," Ibid.

H. G. Worste11, "Mechanical Design of the LASL Linac," Ibid.

J. M. Potter, "A Real-Time Sampling Beam Position Monitor," Ibid.

R. A. Gore, "Los Alamos Meson Physics Facility Control Philosophy," Ibid.

D. C. Hagerman, "805-MHz Power Sources for the LAMPF Accelerator," Ibid.

R. A. Jameson, "Automatic Control of RF Amplified Systems," Ibid.

J. D. Doss, "Fault Protection Circuitry for the LASL 201. $55-\mathrm{MHz}$ Power Amplifiers," Ibid.

J. F. Faulkner and T. J. Boyd, "LAMPF 200-MHz Power Sources," Ibid.

T. G. Van Vessem, "High Duty Factor Floating Deck Modulator for Los Alamos Meson Physics Facility," Itid.

D. A. Swenson, E. J. Schneider, E. A. Knapp, and J . M. Potter, "Pcst-Coupled Drift Tube Linac," Ibid.

J. D. Doss, R. L. Cady, D. C. Hagerman, and T, F. Turner, "80-kV Klystron 'Blocking-Oscillator' Modulator," 10th Modulator Symp. May 21-22, 1968.

D. A. Swenson and R. T. Tregellas, "Description of Drift Tube Linac Equipment Systems," Los Alamos Scientific Laboratory internal report MP-3-20.

R. R. Silber and M. E. Schillaci, "On the Discrepancy Between Soft Pion Predictions and Experiment in Pion Production in Nucleon-Nucleon Collisions," Phys. Rev., Uriv. of Ore. \& LRL, Berkeley (1970). 
G. R. Swain, "Schemes for Restarting or Continuing Operation of the LAMPF Side-Coupled Linac after a Failure in one Power Amplifier," Los Alamos Scientific Laboratory internal report MP-3-31.

C. R. Emigh and R. R. Stevens, Jr., "Polarized Ion Source Requirements," Los Alamos Scientific Laboratory internal report $M P-4 / C R E, R R S-1$.

H. A. Thiessen, "Preliminary Report: LAMPF Switchyard Design," Los Alamos Scientific Laboratory internal report $\mathrm{MP}-4 / \mathrm{T}-1$.

R. R. Silbar, "Primary Bean Transport: First to Second Target," Los Alamos Scientific Laboratory internal report MP-6/RRS-2.

D. A. Swenson and K. R. Crandal1, "Displacement of Equilibrium Orbit by Stray Field," Los Alamos Scientific Laboratory internal report $\mathrm{MP}-3-43$.

H. G. Worstell, "Mechanical Engineering $805 \mathrm{MHz}$ Structures," Los Alamos Scientific Laboratory internal report $M P-3-44$.

D. A. Swenson, "Beam Steering and Position Monitoring in Drift Tube Linac," Los Alamos Scientific laboratory internal report MP-45.

R. L. Gluckstern, "Transverse Beam Blow-Up in Standing-Wave Linacs," Los Alamos Scientific Laboratory internal report MP-DO/1.

R. L. Gluckstern, "High Energy Beam Transport of Intense Beam," Los Alamos Scientific Laboratory internal report MP-DO/3.

M. Jakobson and H. Thiessen, "LAMPF Switchyard Design," Los Alamos Scientific Laboratory internal report MP-4/MJ/HT-1.

M. Jakobson, "A Low Energy Positive Muon Channel," Los Alamos Scientific Laboratory internal report $M P-6 / M J-1$.

D. A. Swenson and R. T. Tregellas, "Description of Cooling Water System for the Drift Tube Linac," Los

Alamos Scientific Laboratory internal report MP-3-48.

D. A. Swenson, "Cavity Power Losses and Stored Energies in the Drift Tube Linac," Los Alamos Scientific Laboratory internal report MP-3-50.

D. W. Weber, "The $805 \mathrm{MHz}$ Rice I/O Chassis," Los Alamos Scientific Laboratory internal report MP-1-842.

R, L. Gluckstern, R. R. Stevens, Jr, , and P. W. Allison, "Coupling Between Transverse Oscillations in Linacs," Los Alamos Scientific Laboratory internal report $\mathrm{MP}-\mathrm{DO} / 2$.

D. A. Swenson and J, Ruhe, "Description of the High Vacuum, Soft Vacuum and Rough Vacuum Systens for the Drift Tube Linac," Los Alamos Scientifi: Laboratory internal report MP-3-42.

L. D. Scott, "Transient Analysis of the Side-Coupled Linac and Drift Tube Linac," Los Alamos Scientific Laboratory internai report MP.3-47.
M. Wilson, "Remote-landling Phi losophy" and Equipment for LASSPF," Los Mlamos Scjentific Laboratory internal report $\mathrm{MP}-6 / \mathrm{MH}-2$.

H. F. Vogel, "Proposal for a Stopped Nuon Channel," Los Alamos Seientific Laboratory internal report MP-6/4V-1.

D. E. Nagle, "Linear Accelerators for Protons." Encycl. Britannica.

J. D. Rogers, W. V. Hassenzahl, H. L. Laquer, and J. K. Novak, "Quadrupole Focusing Magnet," 1968 Sumser Study on Superconducting Devices and Accelerators.

L. Rosen, "Mesons and Medicine." Presentation Sept. 12,1968 .

F. D. Terry, "A Versatile Technique for Interfacing a Control Computer with Renote Data Acquisition and Control Stations Along Particle Accelerators," 19úg Part. Accel. Conf. Published in IEEE Transactions on Nuc1. Science.

D. R. Machen, R. A. Gore, and D. W. Haber, "A Compact Data Acquisition and Control Terminal for Particle Accelerators "" Ibid.

D. T. Van Buren, "A Fast Protect System for Linear Aicelerators," Ibid.

D. E. Nagle, "The Los Alamos Meson Factory--A New' Approach to 01d Problems, Colloquium at Univ. of Kansas, 1968.

H. C. Hoyt, "A Simple Method for Determiring Dimensions of $805 \mathrm{Mc} / \mathrm{sec}$ Shaped Linac Cavities," Los Alamos Scientific Laboratory internal report $\mathrm{MP}-3 / \mathrm{HCH}-1$.

D. A. Swenson, R. T. Tregellas, and S. E. Rislove, "Description of Cooling Water System for the Drift Tube Linac," Los Alamos Scientific Laboratory internal report MP-3-48 (Revised).

R. R. Stevens, Jr, and G. G. Ohlsen, "Transfer Matrix Method for Calculating Spin Aberrations in the Transport of Polarized Ion Beams, int. Symp. on Polarization, Los Alamos Scientific Laboratory report LA-4465.

D. A. Swenson, "Description of the Beam Steering System in the Drift Tube Linac," Los Alamos Scientific Laboratory internal report MP-3-49.

1. Thorn and $M$. Wilson, "Roller Tests," Los Alamos Scientific Laboratory internal report MP-6/LT, MW-1.

J. E. Stoval1, "Emit:ance Growth in a $20 \mathrm{~mA}$ Proton Beam," Los Alamcs Scientific Laboratory internal r:port MP-3-52.

H. F. Voge1, "Muon Origins and Time Structure in the Stopped Muon Channel," Los Alamos Scientific Laboratory internal report $\mathrm{MP}-6 / \mathrm{HV}-2$.

H. Vogel, "Magnetic $\pi \mu$ Separation in the Analyzer of the Stopped Mu Channel, Los Alamos Scientific Laboratory internal report $\mathrm{MP}-6 \mathrm{HV}-3$. 
A. R. Mark, "Primary Heat Transport: Properties of 10 and 6n Systems," Los Mlamos Seicnt ific taboratory ineornal report MF-6/ACH, HRS-1.

3. 3. Sternhein. "7" Absorption Cross Sections and Seutron Distribution Parancers." Los Mamos Scientific Laboratory internal report $\mathrm{sp}-120 / 4$.

I1. A. Thiessen. "Lanll: Switchyard Design II," Los Mlamos Scicntific taboratory internal report Mip-A/IT-2.

1. D. Noss, "M lligh lol tage Rezenerat ive Pulse Modulator," Los Mathos Scientific Lahoratory report LA-4091.

b. Stienson, "Ueseription of the Hean Stcering System in the Drife Tube timac," Los Alamos Scientifis tilloratory internal report $\mathrm{NP}-3-49(R)$.

1. X. Engle. "Geonetrical and Electronngnet is Paraneters of the Accelerating and Coupling Cells of the sos thl: Linte for LwPF," tos Mamos Scientific laboratory intermat report \$P-3-58.

1. x. Engle, "Solution of E Fields from F-Tables of the LAla Code," los Mlanos Scientific Iaborator. internal report $17-5-60$.

4. Trum and 11. Swenson, "beam position and Current "lonitor Systens for the Vrife Jube linac." Los Alanos Scientific Laboratory internil renort NTP-3-61.

4. Trump, "Beam Position p. Current Yonitor Systers for the Side Coupled Linac." tos Mlamos scientific laboratory internal report 14 -3-62.

G. R. Swain, "Drift Tube Linac Hesonanec Control Systen Design Reviex," Los Mamos Scientific Imboratory internal report $91 \mathrm{p}-3-65$.

P. J. Tallerico, "The liffects of Finite Focusing Fields on klystron Performance, 1970 IEEE Int. Elec. Der. Conf.

k. I. Shlacr, "Description of the Hent Steerng Syster for the Side Coupled Linac," Los Alamos Scientific aboratory internal report MP-3-6A.

S. E. Rislove, D. A. Swenson, and K. T. Tregellas, "Description of the Cooling Water System for the Side Coupled Linac, Los Mlamos Seientific Laboratory internal report $\mathrm{MP}-3-65$

E. 3. Schneider, "Description of the Power Supply and Shunt System for the Oundrupole Magnets of the Drift Tube Linac," Los Alamos Scientific Laboratory internal report $\mathrm{MP}-3-66$.

3. R. Ruhe, R. T. Tregellas, and D. A. Swenson, "Description of the Vacuun Systen for the Side Coupled linac," Los Alamos Scientific Laboratory internal report MP-3-67.

W. J. Shlaer and D. A. Swenson, "Description of the Quadrupole Doublet System of the Side Coupled Linac," Los Alamos Scientific Laboratory internal repori MP-3-68.
R. T. Tregellas, "Wescription of the Monitor Loop Systen for the Drift Tube Linac," Los Alamos Scientific laboratory interna: report $\mathrm{MP}-3-69$.

J. E. Stovall, "Drift Tube Linac Error Studies," Los Alamos Scientific Laboratory internal report IT-5- $/$ l.

R. T. Tregellas, "Description of the Frequency Tuner and Field Tilt Systems for the Urift Tube Linac," Los Alamos Scientific Laboratory internal report MTP-.3-7I.

D. A. Shenson, "The Bucket Rotator," Los Alamos Scientific laboratory internal report MP-3-72.

v. H. Itughes, "Stopped Muon Channel," Los Alamrs Scientific Laboratory internal report MP-DO, libi-4.

d. Lundy, "Des ign Revien of Modular Implementution of local Controls." Los Alamos Scientific Laboratory internal report $\mathrm{MP}-1-1901$.

A. P. Conley, "LAUPf Master Timer Design," Los Alanos Scient if ic Laboratory internal report YyP-1-612.

H. Butler, "LAUPF Specification for Digital Control System," Los Mamos Scientific Laboratory internal report IP-1-911.

3. Jakobson and H. Thiessen, "LAMPF Switchyard Design," Los Alamos Scicrific Laboratory internal report $\mathrm{sp}-4 / \mathrm{s} / \mathrm{IT}-1$.

R.R. Stetens, "Design of an Achromatic Inflector for the Dual Beam Transport System in the LANPF Injector," Los Mlamos Scient ific Laboratory internal re. port MP $-4 /$ RRS -4

II. Vogel, "Crossed-field Velocity Separator for FroJucing Transverse-Polari-ed Aluons," Los Alamos Scientific Laboratory internal report MP-6/HN-4.

D. T. Van Burca, "LAMP! Fast Protect System," Los Manos Seientific Laboratory internal report W-1-702 Revised.

T. M. Putnam. "Conceptunl Design of the LAstPF Communication Systen," Los Mamos Scient ific Laboratory internal report $M \mathrm{P}-1-1003$.

J. R. Parker, "Rad-Safe Sustem," Los Alamos Sejentific Laboratory internal report ISP-1-1112.

3. B. Sharp, "The Side-Coupled Linac Temperature Control System," Los Mlamos Scientific Laboratory internal report MP-1-1903.

D. T. Van Buren, "LMJPF Video System," Los Alamos Scientific Laboratory internal report MP-1-2613.

B. L. Hartway, "Engineering Study for the LAMPF Communications System," Los Alamos Scientific Laboratory internal report MP- $\$-1006$.

3. R. Parker, "Radiation Damage in the LAMpF Radiation Environment," Los Alamos Scientific Laboratory internal report MP-1-1115. 
L. X. Engel and D. A. Swenson, "Interpretation of LALA Output," Los Alamos Scientific Laboratory" internal report MP-3-85.

G. R. Swain, "Tuning Equations for Stabilized $(2 \mathrm{~N}-1$; $\pi(2 N)$-Mode Superperiodic Linacs Based on the CoupledResonator Model: Normal $\pi$ - and Sagamore-Mode Operation," Los Alamos Scientific Laboratory internal report MP-3-91.

G. R. Swain, "Illustrated List of Linac Transient Analys is Movies," Los Alamos Scientific Laboratory internal report MP-3-94.

E. J. Schneider, "Description of the Power Supply" and Shunt System for the Quadrupole Magnets of the Drift Tube Linac," Los Alamos Scientific Laborazory internal report MP-3-66.

A. L. LaRotonda, "LAMPF Switchyard," Los Alamos Scientific Laboratory internal report MP-4/ALL-1.

R. Burman and M. Jakobson, "Note on a Low-Energy Pion Channel for LANIPF," Los Alamos Scientific Laboratory internal report $\mathrm{MP}-6 / \mathrm{RB}, \mathrm{MJ}-1$.

V. W. Hughes, S. Ohnuma, K. Tanabe, ard H. Vogel, "Design Status Report for the Stopped Muon Channel," Los Alamos Scientific Laboratory internal report IIP-6/RB, MJ -1 .

S. Sobottka, "Parasite Pion Channels," Los Alamos Scientific Laboratory internal report $M P-D O / 6$.

R. A. Jameson, "Measured Bynamic Performance of 1.25 MW, 805-MHz Klystrons," Tech. Mtg. Elec. Dev. Grp. (1970).

"Can the Reartion $v_{e}+v_{e}+v_{e}+e^{-}$be measured at LAMPF?" Los Alamos Scientific Laboratory internal report $\mathrm{MP}-\mathrm{DO} / \mathrm{JWC}-1$.

D. J. Liska, "Electric Field Measurements of Klystron Cavities," Los Alamos Scientific Laboratory internal report MP-2/DJL-3.

R. R. Silbar, "Waist-to-Waist Transport with One Thin Lerı," Ios Alamos Scientific Laboratory internal report MP-6/RRS-3.

R. R. Silbar, "Comments on Symmetric Triplets," Los Alanos Scientific Laboratory internal report IP $-6 /$ RRS -4 .

K. R. Crandall and D. A. Swenson, "Side Coupled linac Turn-on Problem," Los Alännc Scientific Laboratory internal report MP-3-98.

D. Walker, "Injector Personnel Safety System," Los Alamos Scientific Laboratory internal report I.P - 1-2114.

C. R. Emigh, "Thick Target Bremsstrahlung Theory," Los Alamos Scientific Laboratory report LA-4097.

P. J. Tallerico, "Large Signal Effects in the Multicavity Kiystron," Los Alamos Scientific Laboratory report LA-4389.
H. L. Anderson. D. E. Nagle, and H. A. Thiessen, "Forward Differential Cross Sections for the Reaction $p+p \rightarrow d+\pi^{+}$in the Range 3.4 to $12.3 \mathrm{GeV} / \mathrm{c}$," Los Alamos Scientific Laboratory report $L A-4483$.

T. Purnam and $J$. Parker, "Tests of Combustion Product Detectors in a Radiation Environment., Los A!amos Scientific Laboratory report LA-4148-MS.

R. Stevens et al.," "Depolarization and Emittance Degradation Effects As sociated with Charge Transfer in a Magnetic Field," NIM 73 .

M. Wilson, "Remote Systems Requirements of Accelerators," Nucl. News, Vol. 12, No. 3.

D. J. Liska, "A Simple Description of Combined Precession and Nutation in an $\mathrm{N}$-Member System of Coaxial, Differentially Spinning Bodies," Publ. Mar. 1970 .

"LAME'F Hot Cell Complex," 17th Conf. on Remote Sys. Technology, Nov. 1969.

H. A. Thiessen, "High Resolution Pion and Proton Spectroscopy at LAMPF," 3rd Int. Conf. on Hi-Energy Phys. E Nucl. Struc., Sept. 1969.

R. L. Burman, "Los Alamos Meson Physics Facility," Ibid.

P. Franke and D. Keller, "Shielding. Requirements for a Linear Accel erator," Amer. Soc. of Civil Engineers.

R. J. Macek et al., "Upper Limit on the Structure Dependent Radiation in $\mathrm{K}^{+}+\mathrm{dJ}^{+}+\nu+\delta$, " Hi-Energy Physics Conf., Aug. 1969.

E. A. Knapp, "High Energy Structures," Linear Accelerators.

E. A. Knapp, "High Energy Structures. Fabrication and Tuning," Ibid.

H. Butler, "Use of Computers for Controls," Ibid.

D. E. Nagle, "Meson Production," Ibid.

D. Nagle, "Linear Accelerators for Protons," Encyclopaedia Britannica, 1970.

E. D. Bush, "Fabrication of 201.25-MHz Drift-Tube Linac Quadrupole Magnets, Los Alamos Scientific Laboratory report LA-4276.

M. Wilson, "Flow and Thermal Characteristics of Hydrogen Near its Critical Point in a Heated Cylindrical Tube," Los Alamos Scientific Laboratory report LA-4172.

"Proceedings of the Third LAMPF Users Meeting," Los Alamos Scientific Laboratory report LA-4397.

H. S. Butler, "LAMPF Data Acquisition System," Los Alamos Scientific Laboratory report LA-4504.

P. A. M. Gram, "A Proposal for $\mathrm{P}^{3}$, A : Versatile HighEnergy Pion Beam Facility," Los Alamos Scientific Laboratory report LA-4535. 
"LAMPF Users Handbook," Los Alamos Scientific Laboratory report LA-4586-MS.

S. P. Koczan, "Braze Joint Strength," Los Alamos Scientific Laboratory report LA-4584-MS.

R. A. Jameson and J. D. Wallace, "Dynamic Measurement of Stopband in LAMPF $805 \mathrm{MHz}$ Accelerator Structures at High Power, Using Hybrid Computer Techniques," Los Alamos Scientific Laboratory report LA-4593-MS.

C. E. Groce and K. H. Harper, "Proceedings of the Biomedical Sessions of the Fourth LAMPF Users Meeting," Los Alamos Scientific Laboratory report LA-4602-MS.

V. W. Hughes and H. F. Vogel, "Stopped Muon Channel for LAMPF," Los Alamos Scientific Laboratory report LA-4474-MS.

P. Todd, "The Prognostic Value of Cellular Studies ir Evaluating New Accelerator Beams for Therapy," Los Alamos Scientific Laboratory report LA-4653-MS.

J. L. Detch, Jr, , R. L. Hutson, N. Jarrie, and J. H. Jett, "Accurate Cross Section Measurements of the Reactions $T(p, p) T, T(p, d) D, T\left(p,{ }^{3} H e\right) N$, and $T(\vec{p}, \hat{p}) T$ at 13.600 Mev," Los Alamos Scientific Laboratory report $L A-4576$.

A. K. Kerman, R. R. Silbar, and W. R. Gibbs, "Proceedings of LAMP! Theoretical Study Group," Los Alamos Scientific Laboratory report LA-4637-MS.

L. E. Agnew, K. H. Harper, and B. F. Miller, "Proceedings of the Fourth LAMPF Users Meeting," Los Alamos Scientific Laboratory report LA-4578-MS.

"Quarterly Status Report, Oct. 31, 1970," Los Alamos Scientific Laboratory report. LA-4571-MS.

"Quarterly Status Report, T.!n. 3i, 1971," Los Alamos Scientific Labcratory report 44-4639-MS.

"Quarterly Status Report, Apr. 30, 1971," Los Alamos Scientific Laboratory report LA-4692-MS.

E. Dunn, "Quarterly Statu: Report, July 31, 19;i," Los Alamos Scientific Labo matory report LA-4753-MS.

D. J. Liska, "Investigation of Graphite and CarLon for Stripping the $\mathrm{H}^{-}$Beam in LAMPF," Los Alamos Scientific Laboratory report LA-4795-MS.

R. M. Rolfe, "LAMPF HRS Data Aralysis Including Higher Orde: Corrections," Los Alamos Scientific Laboratory internal report $\mathrm{MP}-7-32$.

R. Macek and B. Maglic, "The Principle of Self-Colliding Orbits and its Possib!e Application to $\pi-\pi$ and $p-p$ Collisions," Particle Accel. 1 (1970).

N. Jaimie, J. H. Jett, J. L. Detch, Jr, and R. L. Hutson, "Proton-Proton Elastic Scattering from 9.6 to $13.6 \mathrm{MeV}$," Phys. Rev. C3 (1971).

H. G. Worste11, "Hydrogen Furnace Brazing of LAMPF Accelerator Structures," Welding J., Mar. 1971.
L. Rosen, "Design and Construction Status of the Los Alamos Meson Physics Facility," Proc. of VII Internat'1. Conf. on Hi-Energy Accel., 1970.

D. E. Nagle, "The Los Alamos Meson Physics Facility," Proc. of the Banff Summer School (Canada), Aug. 1728,1970 .

D. E. Nagle, " $\pi$ Production by 730-MV Protons," Ibid.

D. R. Machen and L. R. Biswell, "CAMAC Sessions at LAMPF," lEEE Trans, on Nucl. Sci., 1971.

L. R. Biswe11, "NOVA Branch Driver Operations," Los Alamos Scientific Laboratory report LA-4682-MS.

R. R. Silbar, "Primary Beam Transport: Experimental Area A," Los Alamos Scientific Laboratory repurt LA-4729-MS.

P. Allison, C. R. Emigh, E. A. Meyer, D. W. Groce, and R. R. Stevens, "Operation of the LAMPF $750-\mathrm{keV}$ Injector," IEEE Trans. on Nucl. Sci., 1971, 1, 126.

P. J. Tallerico, "High Performance Klystrons for Accelerator Operations," Ibid., p. 257.

H. Butler, "Opening Remarks on Computer Control," Ibid., p. 336 .

D. Macinen and L. Biswell, "A Standard Interface Concept for Computer-Controlled Particle Accelerators," Ibid., p. 363.

A. L. Criscuolo, "A Beam Inhibit System for LAMPF," Ibid., p. 367 .

H. Butler, B. Hartway, D. Machen, and T. Put nam, "An Operator's Console for the LAMPF Accelerator," Ibid., p. 419.

E. A. Knapp, "Status Report on LAMPF," Ibid., p. 508.

R. A. Jameson and J. D. Wallace, "Feedforward Control of Accelerator RF Fields," Ibid., p. 598.

D. J. Liska, R. A. Jameson, J. D. Wallace, and J, B. Sharp, "Accelerator Field Measurements at High Power," Ibid., p. 601.

D. A. Sivenson, B. C. Goplen, M. A. Paciotti, and J. E. Stoval1, "Beam Measurements on the First Tank of LAMPF," Ibid., p. 609.

G. R. Swain, R. A. Jameson, E. A. Knapp, D. J. Liska, J. M. Potter, and J. D. Wallace, "Tuning and Pre-Eeam Checkout of $805 \mathrm{MHz}$ Side-Coupled Proton Linac Structures," Ibid., p. 614.

W. V. Hassenzahl, J. D. Rogers, and w. C. Armstrong, "The Effects of Radiation on Superconducting Wire," Ibid., p. 683 .

J. R. Parker, J. H. Richardson, J. D. Oetting, and J. D. Easley, "A Beam-Spill Monitor for LAMPF," Ibid., p. 925 .

A. Harvey and R. D. Turner, "The LAMPF Switchyard Magnets," Ibid., p. 892. 
J. R. Parke:. P. W. Allison, D. W. Mueller, and R. R. Stevens, "Beam Profile Monitoring and Analysis by Television," Ibid., p. 935.

D. J. Liska, "Multipactoring Electron Gun for High Duty Linacs," Proc. IEEE, 59 (1971).

- J. Liska, "Electric Field Measurements in Klystron Cavities;" IEEE Trans. on Electron Devices (1971).

J. L. Detch, R. L. Hutson, N. Jarmie, and J. H. Jett, "Accurate Measurements of the Nuclear Process is $T(p, p) T, T\left(p,{ }^{3} H E\right) n, T(p, d) D$, and $T(p ; p) T$ from 15 te 20 MeV," Phys. Rev. C4, (1971).

I. L. Gammel, M. T. Menzel, and W. R. Wortman, "Bethe-Salpeter Solution for Nucleon-Nucleon Scattering with Pion Exchange," Phys. Rey. D3, (1971).

L. Rosen, "Relevarze of Particle Accelerators to Vitional Goals," Science 173 (1971).

P. J. Tallerico, "Design Considerations for the High Power Multicavity Klystron," IEEE Trans. Electron Devices ED-18, (1971).

R. L. Hutşon, N. Jarmie, J. L. Detch, and J. H. Jett, "p- He Elastic Scattering from 13 to $20 \mathrm{MeV}$," Phys. Rev. C4, (1971).

D. J. Liska, "Investigation of Graphite and Carbon for Stripping the $\mathrm{H}^{-}$Beam in LAMPF," Los Alamus Scientific report $L A-4795-M S$.

"Proceedings of the LAMPF Summer Study Session," Los Alamos Scientific Laboratory report LA-4824-MS.

"LAYPF Neutrino Facility Proposal," Los Alamos Scient ific laboratory report LA-4842-HS.

I. A. Sullivan and C. R, King, "Measurements of Convective Heat Transfer Coefficients for the Subsonic Flow of Hydrogen and Helium on Smooth Tubes at Moderate Surface and Fluid Temperatures," Los Alamos Scientific Laborat'ry report $L A-4882$.

R. Zeidman, "The HRS Spectrometer System 1. Concent and Design," Los Alamos Scientific Laboratory report LA-4773-NS.

H. A, O'Brien, Jr, and M. E. Schillaci, "Isotopic veutron Sources from the Los Alamos Meson Physics Facility," I sotop. Radiat. Techn. 9, (1971).

J. H. Koch, M. M. Srernheim, and J. F. Walker, "Complex rigerivalues of a Coupled-Channels Model," Phys. Rev. C5, (1972).

H. C. Bryant, P. A. Lovoi, and G. G, Ohlsen, "Production of Pulsed Particle Beams by Photodetachment of Negative Hydrogen Ions," Phys. Rev, Lett. 27, $(i \bar{i} i)$.

R. A. Jameson and R. S. Mills, "Two-Dimensional Search and Interpolation on a Distorted Rectangular Grid. Program SI $\phi(; 2), "$ Los Alamos Scientific Laboratory report LA-4B9l-MS.

L. Rosen, M. E. Schillaci, B. J. Dropesky, and H. A. O'Brien, Jr.. "Use of LAMPF for I sotope Production," Los Alamos Scientific Laboratory report LA-4587-MS.
L. R. Biswell and. R. E. Rajala, "A Microprogrammed Branch Driver fcr a PDP-11 Computer," Los Alamos Scientific Laboratory report LA-4916-MS.

J. H. Koch and M. M. Sternheim, "The $K^{-}$-Nucleus Interaction and its Interpretation," Phys. Rev. Lett. 28, (1972).

"Beam Measurements on the High-Intensity Proton Injector of LAMPF," Los Alamos Scientific Laboratory report LA-4961-MS.

"Origins and Histury of LAMPF," Los Alamos. Scientific Laboratory report $\mathrm{LA}-5000$.

"Elastic Pion-Nucleus Scattering for Studies of the Nuclear Surface," Los Alamos Scientific Laboratory report LA-4969-MS.

D. F. Jones and E. R. Martin, "A System for Separating Amplifiers from Wire Proportional Chambers," Nucl. Inst. \& llethods 98, (1972).

E. R. Martin and D. F. Jones, "A Fast Total Information Readout System for Multiwire Proportional Chambers," Ibid.

C. R. Emigh, E. A. Meyer, D. W. Mueller, and R. R. Stevens, "LASL Experience with a Duoplasmatron Feeding a $750 \mathrm{kV}$ 'Exact' Pierce Column," Proc. of the Symp. on Ion Sources (BNL-50310), 1971.

P. W. Allison and C. R. Emigh, "LAMPF $\mathrm{H}^{-}$Ion Source Development," Ihid.

M. M. Sternhęir and R. R. Silbar, "Model for Production of $\pi^{+}$and $\pi^{-}$by Protons from Nuclei," Phys. Rey. C, (1972).

R. L. Burman, "Pion Beams at LAMPF," Internat'l, Sem. on PM Nucl. Interaction, France, (1971).

D. M. Lee, S. Sobottka, and H. A. Thiessen, "A Bifilar Helical Multiwire Proportional Chazber for Position Sensitive Detection of Minimum Ionizing Particles," Los Alamos Scientific Laboratory report LA-4968-MS.

P. R. Bevington, "A Programmer's ruide to the Microprogrammed Branch Driver for the PDP-11," LoS Alamos Scient ific Laboratory report LA-5028-MS.

H. A. Thiessen, "Proposed Fast Electronics for EPICS," Los Alamos Scientific Laboratory report LA-5070-MS.

H. F. Vogel, "Electro-Mechanical Tolerance Requirements for Quadrupoles from the Beam Optical Aberrarions," Los Alamos Scientific Lauuratory report LA-5076-MS.

E. R. Martin and D. F. Jones, "Using Multiwire Proportional Chamber Decoders," Los Al amos Scientific Laboratory report LA-5073-MS.

R. F. Thomas, Jr., "Specifications for CAMAC Subroutines," Los Alamos Scientific Laboratory report LA-50S9-MS. 
H. A. Thiessen and J, P. Egger, "Computer Control of EPICS Beam Line and Spectrometer," Los Alamos Scientific Laboratory Report LA-5092-MS.

R. A. Eisenstein, ?. U. Barnes, and H. A. Thiessen, "A Proposal for EPICS Data Acquisition Software," Los Alamos Scientific laboratcry Report LA-5079-MS.

L. Rosen, "LAMPF, A New Tool for Basic Research and Practical Applications," Int. Conf. Peaceful Uses of At. Energy 1972.

J. H. Koch and M. M. Sternheim, "Models for $\pi^{+}-16_{0}$ at $270 \mathrm{Mev}, "$ Phys. Rev. C.6 (1972).

L. Rosen, "Nuclear Science and its Future," Proc.

Top. Symp. on Nucl. Sci. 1972.

L. S. Kisslinger, "Nuclear Wave Functions from Particle Transfer Data," Los Alamos Scientific Laboratory report LA-5047-MS.

B. Goplen and R. R. Steven:, Jr., "Studies on the Beam Dynamics of the High-Intensity Proton Beam Line in the Injector Complex of LAMPF," Los Alamos Scientific Laboratory Report LA-5046-MS.

D. A. Swenson, "The Trassition Region," Los Alamos Scientific Laboratory Report LA-5049-MS.

W. Dunwoody, R. Rolfe, J. Spencer, R. Stearns, N. Tanaka, and $M$. Thomason, "Magnetic Measurements on the LASL Protctype Magnet," Los Alamos Scientific Laboratory Report LA-5084-MS.

H. A. Thiessen and M. M. KIein, "Spectrometer Design at LASL," Los Alamos Scientific Laboratory Report LA-5072-MS.

D. R. F. Cochran, P. A. Dean, P. A. M. Gram, E. A. Knapp, E. R. Martin, D. E. Nagle, R. B. Perkins, W. J. Shlaer, H. A. Thiessen, and E. D. Theriot, "Cross Sections for $P+$ Nucleus $\rightarrow \pi \pm+\ldots$ for $740-$ MeV Protons," Los Alamos Scientific Laboratory Report LA-5083-NS.

E. R. Martin and D. F. Jones, "Using Multiwire Proportional Chamber Decoders," Los Alamos Scientific Report LA-5073-MS.

H. F. Vogel and R. J. Lari, "Computation of Magnetic End Fields, and Comparison with Measurement," Los Alamos Scientific Laboratory Report LA-5075-MS.

R. Christian and H. F, vogel, "3D Magnetic Field Co putations and Comparison with Measurements," Los Alamos Scientific Laboratory Report LA-5077-MS.

R. A. Eisenstein, P. D. Barnes, and H. A. Thiessen, "A Proposal for EPICS Data Acquisition Softwar:," Los A.lamos Scientific Laboratory Report LA-5079-MS.

D. F. Jones and E. R. Martin, "Using the CA3054 MriPC Amplifier," Los Alamos Scientific Laboratory Report LA-5085-MS.

R. L. Hutson, "Magnet Alignment Study for the LAMPF Bionedical Pion Charnel," Los Alamos Scientific Laboraiory Report LA-5126-MS.
R. L. Hutson, "Projected Multiple Scattering Distributions," Los Alamos Scientific Laboratory Report LA-5I59-MS.

J. H. Koch and M. M. Sternheim, 'ExoTIC: A Computer Code for Energy Levels of Exotic Atoms " Los Alamos Scientific Laboratory Report LA-5110.

J. H. Koch, "Effects of Strongly Absorptive Interactions," Los Alamos Scientific Laboratory Report LA$4980-\mathrm{T}$.

H. F. Vogel, "Permissible Multiple Field Components in Quadrupole Magnets," Los Alamos Scientific Laboratory Report LA-5112-MS

L. Rosen, "Status of Los Alamos Meson Physics Facility," 4th Conf. on High Energy Physics, USSR, Sept. 9-11, 1971 .

L. Rosen, "Kulevance of L: nass," Proc. 1972 Pzoton Linear Accelerator Conference, 0ct. 10-13, 197:, Los Alamos Scientific Laboratory report LA-5115.

D. E. Nagle, "Status and Performance of LAMPF," ibid. K. R. Cranda11, "Transverse Beam Matching Using Wire Scanners," ibjd.

R. R. Stevens, Jr., B. C. Goplen, and J. E. Stovall, "Beam Transport Studies on the Proton Beam Line in the Injector Complex of LAMPF," ibid.

D. W. Mutler, "RMS Enittance and the LASL LAMPF Beam," ibid.

K. R. Cranda 11, R. A. 'lameson, D. 1. Morris, and D. A. Swenson, "The Delta-T Turr-On Procedures," ibid.

E. W. Colst on and V. E. Hart, "Alignment Philosophy, Design, and Techniques Used at LAMPF," ibid.

G. R. Swain, R. A. Jameson, R. Kandarian, D. J, Liska, E. R. Martin, and J. M. Potter, "Cavity Tuning for the LAMPF 805-MHz Linac," ibid.

J. M. Potter and E. A. Knapp, "Bridge Coupler Design and Tuning Experience at Los Alamos," ibid.

P. W. Allison, D. W. Mueller, E. A. Meyer, and R. R. Stevens, Jr., "Status of the LAMPF $\mathrm{H}^{-}$Injector," ibid.

E. D. Bush, Jr., and R. L. Rhorer, "Construction of Alumina Insulated Bending Magnets for LAMPF," ibid.

R. A. DeHaven, "Computer Controi of LAMPF's 201.25MHz RF," ibid.

V. E. Hart and E. W. Colston, "Installation and Al ignment of LAMPF $201.25-\mathrm{MHz}$ and $805-\mathrm{MHz}$ Linac Tanks," ibid.

D. W. Mueller, E. A. Meyer, R. R. Stevens, Jr., B. C. Goplen, M. A. Paciotti, and C. K. Emigh, "Operation and Performance of the High Intensity Proton Injector of LAMPF," ibid. 
J. R. Parker, J. H. Richardson, and J. D. Easley, "A New Beam Spill Control System for LAMPF," Proc, 1972 Proton Linear Accelerator Conf., Oct. 10-13, 1972, Los Alamos Scientific Laboratory report LA-5115.

E. J. Schneider, "Ripple current and Flux in Mineral Insulated Magnets," ibid.

J. E. Spencer and H. A. Tniessen, "Some High Resolution Techniques for Use with Negative Ion Beams," ibid.

J. E. Stoval1, "An Automated Admittance Measurement at LAMPF," ibid.

P. J. Tallerico, "The LAMPF KJystron Repair Facility," ibid.

H. F. Vogel and J. J. Rosentha1, "Cement Potted Coils for Muon Channel Magnets," ibid.

M. J. Morris and J. R. Parker, "High Intensity Electron Accelerator Radiation Hazards," Int l. Conf. Protection Accel. \& Space Radiation 1971.

N. R. F. Cochran, P. N. Dean, P. A. M. Gram, E. A. Kuapp, E. R. Martin, D. E. Nagle, R. B. Perkins, W. J. Shlaer, H. A. Thiessen and E. D. Theriot, "Production of Charged Pions by $730 \mathrm{M} M \mathrm{eV}$ Protons from Hydrogen and Selected Nuclei," Phys. Rev. D6 (1972).

D. M. Lee, S. E. Sobottka and H. A. Thiessen, "A Bifilar Helical Multiwire Proportional Chamber for Position Sensitive Detection of Minimum Ionizing Particles," Nuc1. Instr. \& Methods 104 (1972).

E. A. Knapp, "Los Alamosskaia Mezonnaia Fabrika," Moscow 1970 .

R. J. Jacob and G. E. Hite, "Extension of Boundary Dispersion Relations," Phys. Rev. D6 (1972).

R. L. Burman, "Pion Beams at LAMPF," Froc. Int. Sem. on $\mathrm{P} i$-Meson Interactions (1971).

R. L. Fulton, "Mechanical Design of the LAMPF LowEnergy Pion Channel," Los Alamos Scientific Laboratory report LA-5222-MS.

J. S. O'Connell, "Neutrino Disintegration of the Deutaron at LAMPF Energies," Los Alamos Scientific Laboratory repurt LA-5175-MS.

D. M. Lee, S. E. Sobottka and H. A. Thiessen, "Proportional Chambers with Monofilar Helical Cathodes for High Spatial Resolution," Los Alamos Scientific Laboratory report LA-5195-MS.

5. C. Hagerman, "LAMPF Operation," Proc. 6th Ntg. of LAMPF Users," Los Alamos, NM, Nov. 8-9, 1971, Los Alamos Scientific Laboratory report LA-5190-C.

R. E. Mischke, "LAMPF Electronics and Equipment Pool," ibid.

E. Rodgers, "Biomedical Channel," ibid.

L. Rosen, "Status of LAMPF," ibid.

L. Rosen, "Some Medical Applications of Accelerators," 1972 Nucl. Sci. Symp. Dec. 5-8, 1972, Miami, FL, lEEE Trans. Nucl. Sci. NS-20, No. 1 (1973).
S. Dhawan and R. F. Thomas Jr., "Standard Software for CAMAC," ibid.

L. Bidwell, D. Machen, J. Potter, R. Rajala and R. Thomas, "A Long Distance CAMAC Branch lia DataLink and Microprogrammed Branch Driver," ibid.

L. Rosen, "The C. P. Anderson Yeson Physics Facility," Proc. Nat. Acad. Sci. 70 (1973).

K. Hlalbach, "Some Eddy Current Effeats in Solid Core Magnets," Nuc1. Instr. \& Methods 107 (1973).

G. J. Igo, N. Tanaka and H. A. Thiessen, "Iligh Resolution Proton Spectrometer at LAMPF," Proc. of the 4th Int. Conf. on Ili Energy Physies USSR (1971).

R. F. Thomas, $\mathrm{Jr}$, "Programing the $\$ 18 \mathrm{D}$ Model 1 ," Los Alamos Scientific Laboratory report LA-5302-115.

J. Amann, "LAMPF Data Handling Workshop; introduction to PDF-11 DOS-8," Los Alamos Scientific Laboratory report $L A-5343-\mathrm{MS}$.

J. J. Reidy and H. B. Knowles, "Physics Input to Negative Pion Therapy," Los Alamos Scientific Laboratory report LA-5300-MS.

E. D. Arthur, "A Study of the $\left(\pi^{+}, p\right)$ and $\left(\pi^{+}, P p\right)$ Reactions on Selected Light Nuclei," Los Alainos Scientific Laboratory report LA-5230-T.

M. D. Thomason, "Cylindrical Point Coils for Magnetic Field Mapping," Los Alamos Scientific Laboratory report LA-5304-MS.

E. Rodgers, "Uni form Dose Distribution with Moving Negative Pion Beams," Los Alamos Scientific Laborztory report LA-5326-MS.

J. P. Egger, "LAMPF Data llandling Workshop; sntroduction to CAMAC," LOS Alamos Scientific Laboratory report LA-5307-MS.

P. A. Thompson, "Heating of Target Cel1 Components by the LAMPF Proton Beam," Los Alamos Scientific Laboratory report LA-5348-MS.

L. Rosen, "Science and Socity; A Complex Interaction," The Physics Teacher, 2 (1973).

J. D. Doss, W. S. Edwards and C. W. McCabe, "A New Electrosurgical Coagulating-Cutting Forcepts," Surgery 74, No. 3 (1973).

L. Rosen, "LAMPF: Exploring the Medical Pctentials of Negative Pions and Negative Muons," Nucl. News 15 (1973).

E. E. Casperson, T. W. Crane, V. W. Hughes, H. F. Kaspar, P. Souder, R. D. Stambaugh, P. A. Thompson, H. Orth, G, zu Putlizz, and A. B. Denison, "Initiation of Muonium Magnetic Resonance Studies at LAMPF," Proc, 4 th Intern. Conf. on Atomic Physics, Heidelberg, July 22-26, 1974 .

P. Souder, D. E, Casperson, T. W. Crane, V. W. Hughes, H. F. Kaspar, R. D. Stambaugh, P. A. Thompson, H. Orth, G. zu Putlitz, A. B. Denison, and $M$. Camani, "Depolarization of Negative Muons in Helium Gas; Search for Muonic Helium Atom," ibid. 
hi. I. Ilassenzah1, U. Laquer, J. k. Vovak, J. U. Rogers and R. W. Stokes, "Superconduct ing Quadrupole loublet for l,AMPF Secondary Bean Lines," 3rd Int. conf. on Magnet Tech., Ger any $(1970)$.

li. 1. Hassenzah1, J. D. Rogers, H. L. Laquer and Ii. C. Armstrong, "Radiation Damage in Niobiumrjtanium Superconducting lires," ibid.

11. F, logel, "liffects of Ai" Intense Flux of Fast and Slow leutrons on the Yagnet P.operties of Steel," ibid.

H. F. loge 1, "p zrturtations froin Steel Environment close to a C Magnet," ibid.

A. Harrey, "Radiation !lardened :lagnets Lsing Mineral Insulated Conductors," Los Alamos Scientific Laboratory report l.A-5306-3is.

5. P. Koczan, R, 1. Rhorer, G. Suazo and H. G. liorstel1, "lydrogen atmosphere furnace Brazing and Snldering Gien Production Opcration at LALPF," Los Alamos Scient ific Laboratory report LA-5216.

1.. R. Biswell and D. T. Lan Buren, "Sola Branchlriver Operation," Los Alamos Scient ific Laboratory report 1.4-5.430.45.

Y. Leon and R. Seki, "Suczear Yot ion Correction to lixotic Atom Level hidths," Phys. Letter $468(10 / 73)$.

ii. J, Shlaer, "PIPLAY, A Treatmentoplanning Code for Pions," Los Alamos Scientific Laboratory report LA$5.45 .5-1.45$.

K. R. Lrandall, 'TRACE, An Interact ive Beam-Transport Program," Los Alamos Scient ific laboratory report LA5.3.3.

J. K. lialbig, F. K. Wiohn, if. L. Talbert, Jx. t. J.

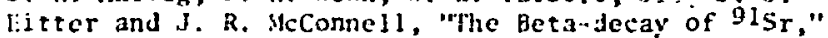
Xucl. Fhys. A, $203(1973)$.

F. K. Kohn, J. K. Halbig, K. L. Talbert, Jr., and J. R. stcconnell," Phys. Rev. C, 7 (1973).

P. J. Tallerico, "The Effects of Load Mismatch on the High-Power lfulticavity k?vstron," Proc. 1973 Eur Ificrowave Conf., Belgi un $(9 / 4-7 / 73)$.

P. J. Tallerico, R. L. Cady and J. D. Doss, "The Design and Performance of the LAMPF I-1/4 kN Klystron Modulator," 1EEE Conf. Record of 197311 th Mod. Symp., NY $(9 / 73)$.

E. R. Flynn, S. Orbesen, N. Stein, H. A. Thiessen, D. M. Lee and S. E. Sobottka, "Applitution of the Helical Cathode Proportional Chamber to Yagnetic Spectrographs for Nuclesr Reaction Studies," Nucl. Instr. \& Methods 111 (1973).

N. Tanaka, M. M. Castro, R. H. Milburn and W. B. Richards, "Recoil-Proton Polarization in NeutralPion Photoproduction between 900-1800 MeV," Phys. Rev. D, 8, (1973).

R. A. Gore and D. R. Machen, "The Use of Minicomputers in Particle Accelerators," Proc. of IEEE, Vol 61 (November 1973).
M. Leon and R. Seki, "Atomic Capture of legative Mesons," Phys. Rev. Lett. 32 (1974).

11. C. Hagerman, "Operating Results on the 800-Hel" Protos Linac at the Los Alamos Scientific Laboratory," IEli: Trans. Sucl. Sci. $\lambda \mathrm{S}-20$ Xo. 3 (1973).

R. L. Hartway, "A Compact Programable Control Panel for Computer Control Systems," ibid.

R. L. Hartway, J. Bergstein and C. 4. Plopper, "The Central Control Room Mar-Machine Interface at LASPF," ibid.

D. R. Mlachen and J. M. Potter, "The Satellite Minicomputer -- A Practical Solution to Accelerator Control," ibid.

J. R. Yachen, "bigital Filter Lesign for Accelerator Dat a and Control Systems," ibid.

(1) Y. Lee, S. E. Sobottka and H. A. Thiessen, "Proportional Chambers with Monofilar Helical Cathodes ior lligh Spatial Resolution," Nucl. Instr. E lethods $10.3(1975)$.

A. $\therefore$. Anderson, R. J, Barrett and E. A. Wadlinger, "Lok Deadtime Delay Line Readout for Position-Sensitive Proportional Counters," Los Alamos Scientific Laborat ory report LA-552.3-MLS.

J. J. Jarner, R, C. Haight, J. E. Simmons, J. C. Martin and T. K. Donoghue, "Analyzing-power Measurements in the ${ }^{3} H\left(p, n j{ }^{3}\right.$ He Reaction," Ph $\because s$. Rev. $C, 9$ (1974).

G. E. Hite and H. J. K. Muller-Kirsten, "Fixed Poles and Compusiteness," Phys. Rev. D 9 (1974).

J. J. Jarmer, J. C. Mart in, G. G, Ohlsen, G. C.

Salzman and J. E. Simmons, "Longitudinal Polarization Transfer in the $T(p, n){ }^{3}$ He Reaction," Phys. Lett. 48B (1974).

H. 4. Bethe and M. B. Johnson, "Theory of Pion Scattering by Nuclei : 1 " Los Alamos Scientific Laboratory report LA-550.3.

M. Leon and R. Seki, "Determination of the Neutron Halo from Antiproton Absorption," Phys. Lett. 48B (1974).

S. Merlan, H. Kasha, P. Wanderer, R, Adair, C. Ankenbrandt, R. Larsen, L. Leipuner, L. Smith, $R$. Stefanski and $F$. Shively, "Determination of $K ;$ Form Factors from Measurements of Decay Correlations and Mon Polarizations," Phys. Rev. D 9 (1974).

J. D. Bowman, L. Ley and $B$ Richter, "Electromagnetic Transition Rates in $215_{\mathrm{Po}}$ " Nucl. Phys. A 220 (1974).

R. L. Hutson, "In Vivo Tissue Agai ysis Using MuMesic X-Rays," Trans. of American Nuclear Soc. 18 June 1974, pp. 98-99.

Sally Shlaer, "Ar MBD Primer," Los Alamos Scientific Laboratory report LA-5511-MS, Aug. 1974.

Raymond A. Gore and Donald R. Machen, "Minicomputer Applications at LAMPF," 1973 IEEE Region-Six Conf. U.S. Western Region, pp. 112-18. 
ㄱ. U. Cuoper, "Time-of-Flight Measurements at the LAMPF Low-Energy Pion Channel," Los Alamos Scientific report LA-5529-MS March 1974).

R. D. Stambaugh, D. E. Casperson, T. W. Crane, V. W. Hughes, P. Souder, H. F. Kaspar, P. A. Thompson,

H. Orth, and G. zu Putlitz, "Muonium Formation in

Noble Gases and Noble Gas Mixtures," Phys. Rev. Lett. 33, 568-571 (1974).

H. S. Butler, "Performance of LAMPF Computer Control System," Proc. III Al1-Union Nati. Conf. on Particle Accelerators, Moscow, USSR, October 2-4, 1972,

Voi. II, pp. 46-55.

DIS GOV:RNMENT PAINTING OFFICE 1974-677.177/32 